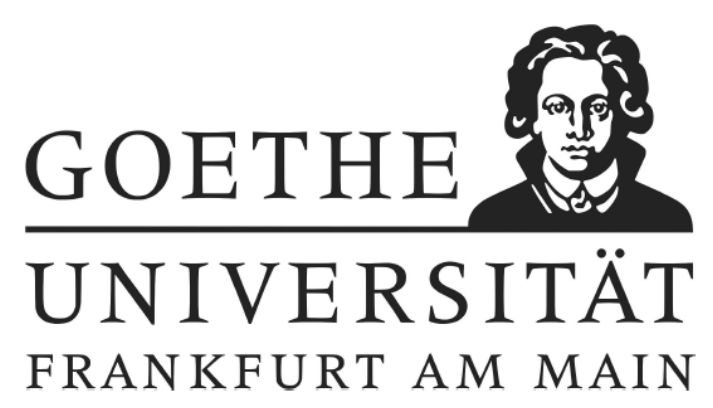

\title{
Evaluation of cell death pathways in cancer and inflammation
}

\author{
Dissertation \\ zur Erlangung des Doktorgrades \\ der Naturwissenschaften \\ vorgelegt beim Fachbereich 14 Biochemie, Chemie und Pharmazie \\ der Johann Wolfgang Goethe-Universität \\ in Frankfurt am Main \\ von \\ Anne-Kathrin Knuth \\ aus Frankenberg (Eder)
}

Frankfurt am Main, 2021

(D30) 
Vom Fachbereich Biochemie, Chemie und Pharmazie (FB14) der Goethe-Universität Frankfurt am Main als Dissertation angenommen.

Dekan:

1. Gutachter:

2. Gutachter:

Datum der Disputation:
Prof. Dr. Clemens Glaubitz

Prof. Dr. Volker Dötsch

Prof. Dr. Andreas Weigert 


\section{Table of contents}

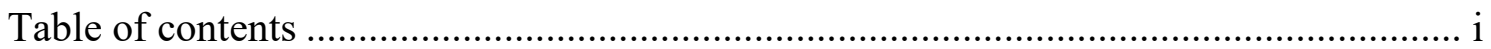

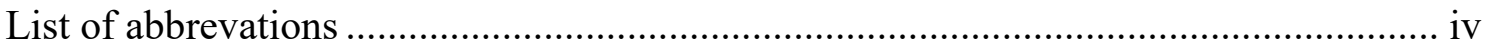

List of figures .............................................................................................. vii

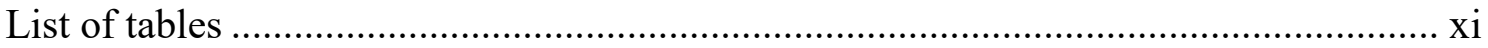

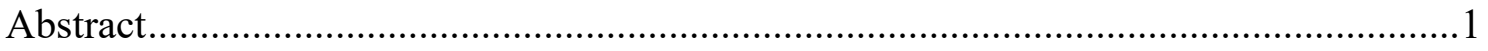

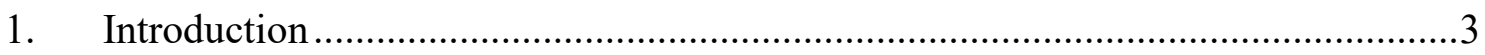

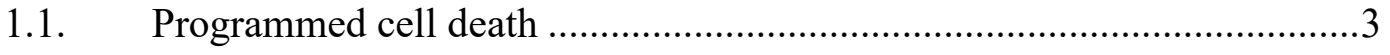

1.1.1. Apoptosis ............................................................................. 3

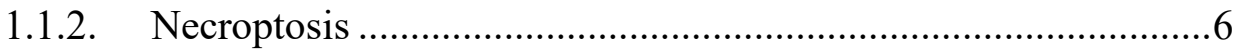

1.1.3. Dimerizer-induced cell death............................................. 7

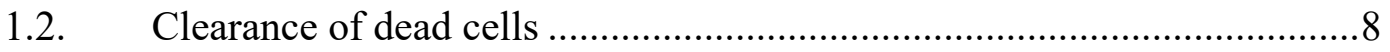

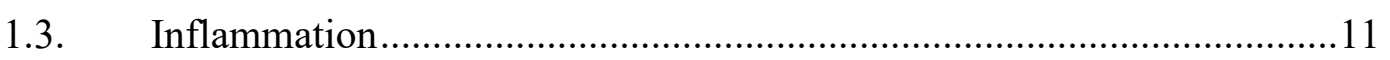

1.3.1. Onset of inflammation ........................................................... 11

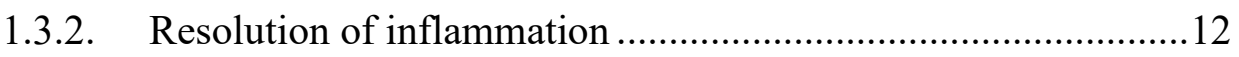

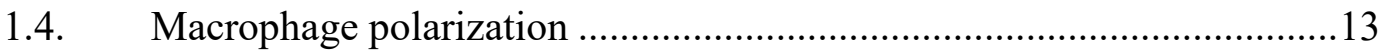

1.5. Proliferation of macrophages and activation of the cell cycle .................14

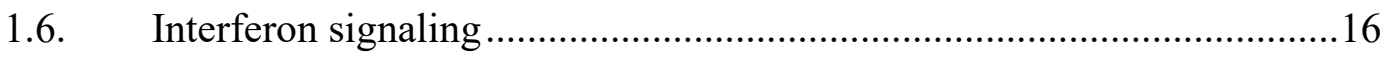

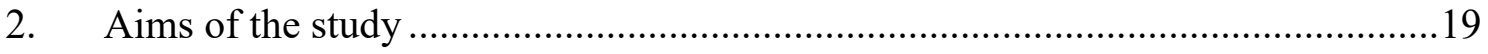

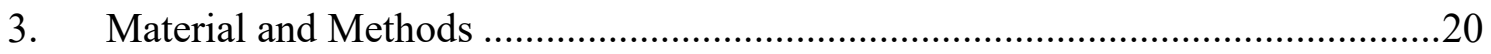

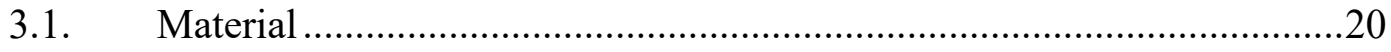

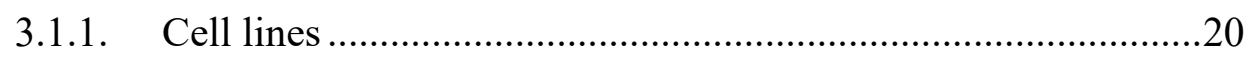

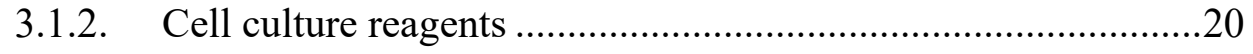

3.1.3. Drugs and inhibitors ..........................................................21

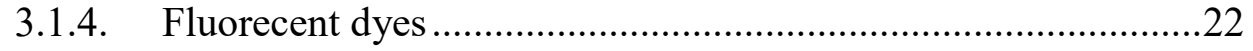

3.1.5. Antibodies .....................................................................22

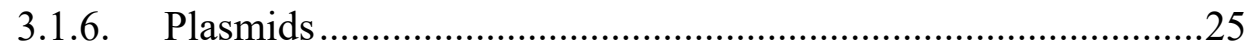

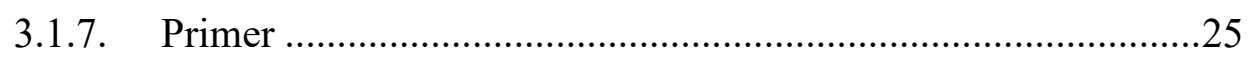

3.1.8. Small-interfering RNAs (siRNAs) ….....................................26

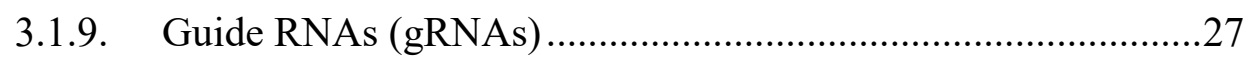

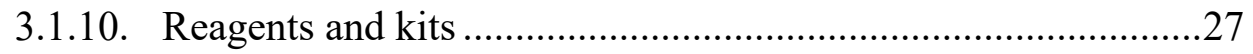

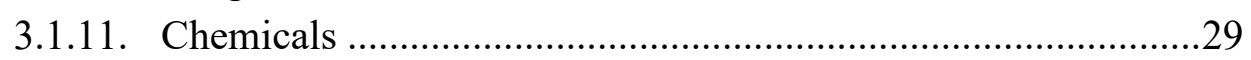

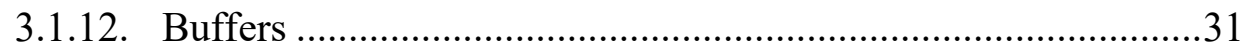

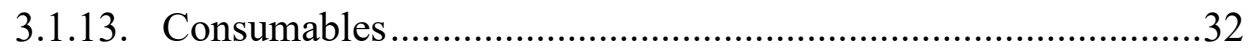

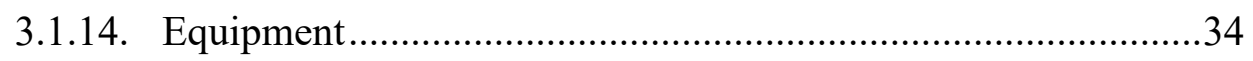

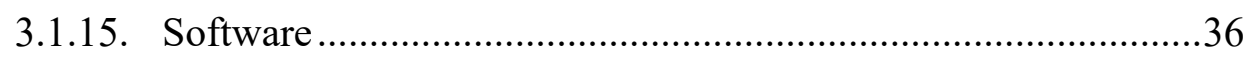

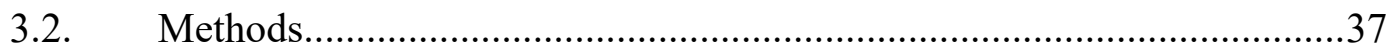




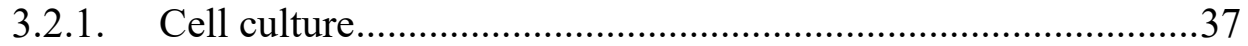

3.2.2. Treatment of cells in vitro ......................................................... 38

3.2.3. Isolation of peritoneal macrophages .........................................38

3.2.4. Transient transfections using siRNA ........................................39

3.2.5. Generation of genetically modified cell lines .............................39

3.2.6. In vivo experiments ...........................................................40

3.2.7. Cell death and cell viability assays ..........................................43

3.2.8. Actinomycin D chase experiments .......................................44

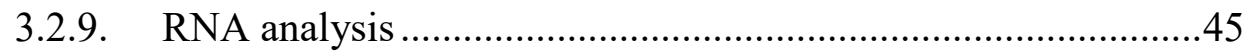

3.2.10. Western Blot analysis .........................................................46

3.2.11. Cytokine and chemokine analysis ...........................................46

3.2.12. Caspase $3 / 7$ activation assay...............................................4

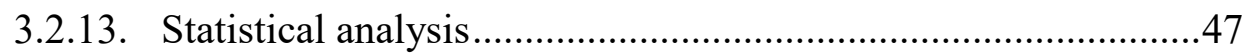

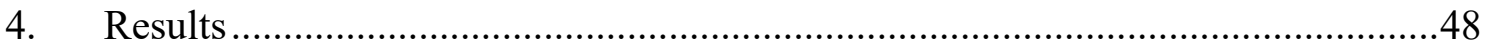

4.1. Part I: IFNs induce MLKL expression in cancer cells..........................48

4.1.1. IFN $\gamma$ together with pan-caspase inhibitor zVAD.fmk induced necroptosis and reduced cell viability of cancer cells................48

4.1.2. IFNs increase MLKL mRNA and protein levels in different cancer cell lines over time ...................................................50

4.1.3. Inhibition of transcription prevents IFN $\gamma$-induced MLKL

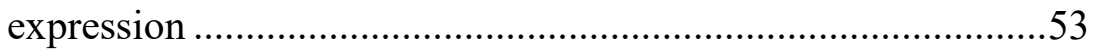

4.1.4. MLKL upregulation is indepent of activation of caspases .......54

4.1.5. IRF1 and STAT1 contribute to MLKL up-regulation by IFN $\gamma .55$

4.2. Part II: Effect of dying cells on immune cells and resolution of

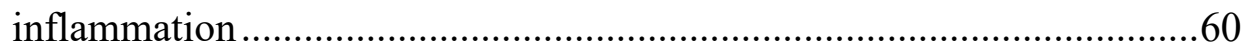

4.2.1. Generation of inducible cell death systems ............................60

4.2.2. Establishing of a co-culture system of dying cells and peritoneal macrophages

4.2.3. RNA sequencing of macrophages co-cultured with dying cells 69

4.2.4. Apoptotic cells induce proliferation of macrophages ................77

4.2.5. Necroptotic cells affect chemokine profil in macrophages .......79

4.2.6. Effect of dying cells on resolution of inflammation in vivo ......82

5. Discussion

5.1. Part I: IFNs induce MLKL expression in cancer cells...........................99

5.1.1. MLKL is transcriptionally upregulated upon IFN signaling .....99

5.1.2. IFN-dependent upregulation of MLKL can be used to sensitize apoptosis-resistant cancer cells towards necroptosis ...............100

5.2. Part II: Effect of dying cells on immune cells and resolution of

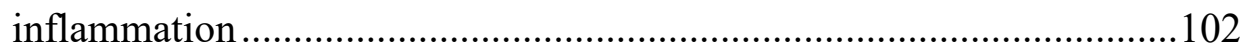

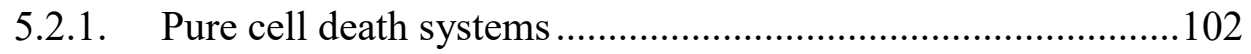

5.2.2. Co-culture of dying cells with peritoneal macrophages ..........104 
5.2.3. Apoptotic cells induce proliferation of peritoneal macrophages

5.2.4. Necroptotic cells change the chemokine profile of macrophages and recruitment of neutrophils.............................................. 108

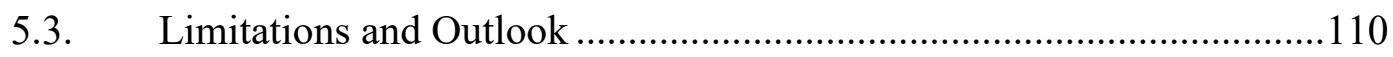

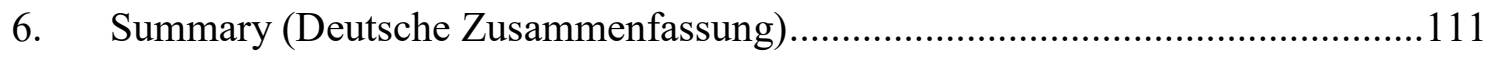

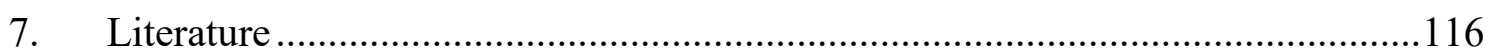

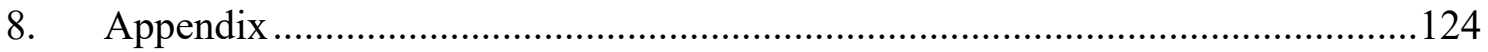

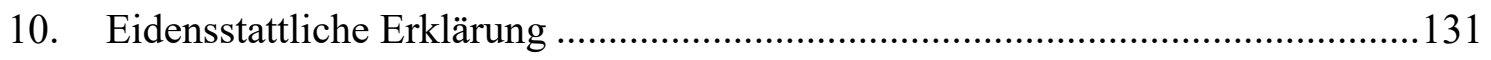




\section{List of abbrevations}

APAF1

APS

ATCC

ATP

BAK

BAX

BCL2

BCL-X

BID

BSA

$\mathrm{CaCl}_{2}$

CBA

CCL

$\mathrm{CDK}$

cDNA

CXCL

CYLD

DAMP

DBD

Dimerizer

DISC

DMEM

DMSO

DNA

DPBS

DR

DSMZ

DTT

ECAR

EDTA

ER

ESCRT

FADD

FAS

FCCP

FCCP

FCS

FITC

FKBP
Apoptotic protease activating factor 1

Ammonium persulfate

American Type Culture Collection

Adenosine triphosphate

Bcl-2 homologous antagonist/killer

Bcl-2-associated $\mathrm{X}$ protein

B-cell lymphoma 2

B-cell lymphoma-extra large

BH3 interacting-domain death agonist

Albumin fraction $\mathrm{V}$

Calcium chloride

Cytometric bead array

Chemokine (C-C motif) ligand

Cyclin-dependent kinase

complementary DNA

Chemokine ( $\mathrm{C}-\mathrm{X}-\mathrm{C}$ motif) ligand

Cylindromatosis

Danger-associated molecular pattern

DNA binding domain

B/B-Homodimerizer

Death-inducing signaling complex

Dulbecco's Modified Eagle Medium

Dimethyl sulfoxide

Deoxyribonucleic acid

Dulbecco's phosphate-buffered saline

Death receptor

Deutsche Sammlung von Mikroorganismen und Zellkulturen

Dithiothreitol

Extracellular acidification rate

Ethylene diamine tetraacetic acid

Endoplasmic reticulum

Endosomal sorting complexes required for transport

Fas-associated protein with death domain

Fas receptor

Carbonyl cyanide 4-(trifluoromethoxy)phenylhydrazone

Cyanide 4-(trifluoromethoxy)phenylhydrazone

Fetal calf serum

Fluorescein isothiocyanate

FK-506 binding protein 


\begin{tabular}{|c|c|}
\hline FLIP $_{L}$ & FLICE-like inhibitory protein large \\
\hline FV & FKBP F36V mutant \\
\hline G phase & Gap phase \\
\hline GAF & IFN $\gamma$-activated factor \\
\hline GAS & Gamma-activated sequence \\
\hline GFP & Green fluorescent protein \\
\hline GM-CSF & Granulocyte-macrophage colony-stimulating factor \\
\hline GOrilla & Gene Ontology enrichment analysis and visualization tool \\
\hline gRNA & Guide RNA \\
\hline GSEA & Gene set enrichment analysis \\
\hline GSK & GSK'872 \\
\hline hCasp8 & Human caspase 8 \\
\hline $\mathrm{HCl}$ & Hydrochloric acid \\
\hline HIF-1 $1 \alpha$ & Hypoxia-inducible factor 1 -alpha \\
\hline HMGB1 & High mobility group box 1 \\
\hline HOIL-1L & Haem-oxidized IRP2 ubiquitin ligase-1 \\
\hline HOIP & HOIL-1-interacting protein \\
\hline HRP & Horseradish peroxidase \\
\hline IAD2 & IRF-association domain \\
\hline IAP & Inhibitor of apoptosis \\
\hline IFN & Interferon \\
\hline IFNR & Interferon receptor \\
\hline IL & Interleukin \\
\hline IRE & IFN-stimulated response element \\
\hline IRF1 & Interferon regulatory factor 1 \\
\hline IRF-R & IRF-binding element \\
\hline ISG & Interferon-stimulated gene \\
\hline ISGF3 & ISG factor 3 \\
\hline JAK & Janus kinase \\
\hline $\mathrm{KCl}$ & Potassium chloride \\
\hline $\mathrm{KH}_{2} \mathrm{PO}_{4}$ & Potassium dihydrogen phosphate \\
\hline LPC & Lysophosphatidylcholine \\
\hline LTB4 & Leukotriene B4 \\
\hline LUBAC & Linear ubiquitin chain assembly complex \\
\hline M phase & Mitotic phase \\
\hline MAPK & Mitogen-activated protein kinase \\
\hline MCL-1 & Myeloid cell leukemia 1 \\
\hline M-CSF & Macrophage colony-stimulating factor \\
\hline MDM & monocyte-derived macrophage \\
\hline MEF & Murine embryonic fibroblast \\
\hline MerTK & Tyrosine-protein kinase Mer \\
\hline $\mathrm{MgCl}_{2}$ & Magnesium chloride \\
\hline $\mathrm{MgSO}_{4}$ & Magnesium sulfate \\
\hline
\end{tabular}




\begin{tabular}{|c|c|}
\hline MLKL & Mixed lineage kinase domain-like \\
\hline MOMP & Mitochondrial outer membrane permeabilization \\
\hline mRIPK3 & Murine RIPK3 \\
\hline mRNA & Messenger RNA \\
\hline MTT & Thiazolyl Blue Tetrazolium Bromide \\
\hline MФ & Macrophage \\
\hline $\mathrm{Na}_{2} \mathrm{HPO}_{4}$ & Disodium phosphate \\
\hline $\mathrm{NaCl}$ & Sodium chloride \\
\hline $\mathrm{NaHCO}_{3}$ & Sodium bicarbonate \\
\hline $\mathrm{NaOH}$ & Sodium hydroxide \\
\hline Nec-1s & Necrostatin-1s \\
\hline $\mathrm{NF} \kappa \mathrm{B}$ & Nuclear factor kappa-light-chain-enhancer of activated B-cells \\
\hline $\mathrm{NH}_{4} \mathrm{Cl}$ & Ammonium chloride \\
\hline NK & Natural killer \\
\hline NLS & Nuclear localization signal \\
\hline NO & Nitric oxide \\
\hline NSA & Necrosulfonamid \\
\hline NSAID & Non-steroidal anti-inflammatory drug \\
\hline OCR & Oxygen consumption rate \\
\hline PAMP & Pathogen-associated molecular pattern \\
\hline PBS & Phosphate buffered saline \\
\hline PBS-T & PBS-Tween \\
\hline PCA & Principal component analysis \\
\hline PCR & Polymerase chain reaction \\
\hline $\mathrm{PE}$ & Phycoerythrin \\
\hline PGE2 & Prostaglandin E2 \\
\hline PI & Propidium iodide \\
\hline PIC & Protease Inhibitor Cocktail \\
\hline PKR & Protein kinase $\mathrm{R}$ \\
\hline pMLKL & Phosphorylated MLKL \\
\hline PMSF & Phenylmethylsulfonyl fluoride \\
\hline Polybrene & Hexadimethrine bromide \\
\hline $\operatorname{PPAR} \gamma$ & Peroxisome proliferator-activated receptor gamma \\
\hline PPIase & Peptidyl-prolyl isomerase \\
\hline PRR & Pattern recognition receptor \\
\hline PS & Phosphatidylserine \\
\hline pSTAT1 & phosphorylated STAT1 \\
\hline RHIM & RIP homotypic interaction motif \\
\hline RIPK1 & Receptor-interacting serine/threonine-protein kinase 1 \\
\hline RIPK3 & Receptor-interacting serine/threonine-protein kinase 3 \\
\hline RNA & Ribonucleic acid \\
\hline ROS & Reactive oxygen species \\
\hline
\end{tabular}




$\begin{array}{ll}\text { RPMI } & \text { Roswell Park Memorial Institute Medium } \\ \text { RT-PCR } & \text { Real-time PCR } \\ \text { S phase } & \text { Synthetic phase } \\ \text { S1P } & \text { Sphingosine-1-phosphate } \\ \text { SDS } & \text { Sodium dodecyl sulfate } \\ \text { SEM } & \text { Standard error of the mean } \\ \text { SHARPIN } & \text { SHANK-associated RH domain-interacting protein } \\ \text { siRNA } & \text { Small-interfering RNA } \\ \text { Smac } & \text { Second mitochondria-derived activator of caspases } \\ \text { SPM } & \text { Specialised pro-resolving mediator } \\ \text { STAT1 } & \text { Signal transducer and activator of transcription 1 } \\ \text { TAM } & \text { Tumor-associated macrophage } \\ \text { TEMED } & \text { Tetramethylethylendiamine } \\ \text { TGF- } \beta & \text { Transforming growth factor beta } \\ \text { TIM } & \text { T-cell immunoglobulin and mucin-domain containing } \\ \text { TLR } & \text { Toll-like receptor } \\ \text { TNF } & \text { Tumor necrosis factor } \\ \text { TNFR1 } & \text { TNF receptor-1 } \\ \text { TRADD } & \text { TNF receptor type1-associated death domain } \\ \text { TRAF2 } & \text { TNF receptor associated factor 2 } \\ \text { TRAIL } & \text { TNF-related apoptosis-inducing ligand } \\ \text { t-SNE } & \text { t-distributed stochastic neighbor embedding } \\ \text { TYK2 } & \text { Tyrosine kinase 2 } \\ \text { UTP } & \text { Uridine-triphosphate } \\ \text { zVAD.fmk } & \text { N-benzyloxycarbonyl-Val-Ala-Asp (O-Me) fluoromethylketone }\end{array}$




\section{List of figures}

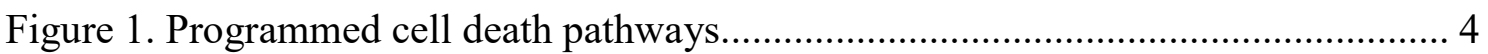

Figure 2. Chemical structure of the homodimerizer AP20187 (B/B homodimerizer),.... 8

Figure 3. Factors mediating clearance of dying cells. ............................................... 9

Figure 4. Time line of inflammation and its resolution............................................... 12

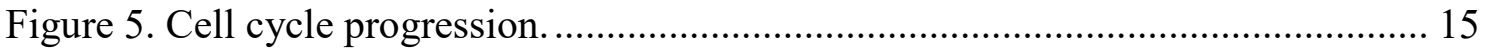

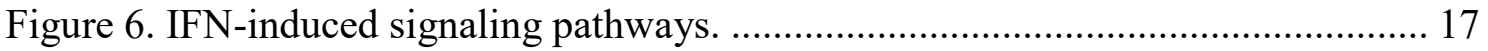

Figure 7. Gating strategy of immune cells isolated from mice. ................................. 42

Figure 8. Treatment with IFN $\gamma$ and zVAD.fmk induced cell death in EFM-192A cells but not in HeLa cells 48

Figure 9. IFN $\gamma$ and zVAD.fmk treatment for different periods of time led to cell death in EFM-192A cell.

Figure 10. IFN $\gamma$ and zVAD.fmk reduced cell viability in EFM-192A and HeLa cells.. 49

Figure 11. IFN $\gamma$ induced necroptosis in EFM-192A cells. 50

Figure 12. IFN $\gamma$ treatment increased MLKL and IRF1 mRNA and protein level over time.

Figure 13. Type 1 and 2 IFNs increased expression of MLKL in different cell lines.... 52

Figure 14. mRNA level of RIPK1 and RIPK3 after IFN $\gamma$ treatment........................... 53

Figure 15. Inhibition of transcription by Actinomycin D inhibts MLKL upregulation. 54

Figure 16. Inhibition of caspases had no effect on IFN $\gamma$-dependent upregulation of

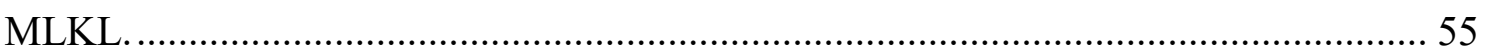

Figure 17. Single knockdown of IRF1 had no effect on MLKL upregulation............... 56

Figure 18. Single knockdown of STAT1 had no effect on MLKL upregulation........... 57

Figure 19. Double knockdown of IRF1 and STAT1 abolished MLKL upregulation.... 58

Figure 20. Knockout of IRF1 prevented IFN $\gamma$-induced MLKL upregulation.

Figure 21. NIH 3 T3 cells stabily expressing FV-hCasp8 or mRIPK3-FV were generated

Figure 22. Expression of FV-hCasp8 and mRIPK3-FV was confirmed at higher passages.

Figure 23. Optimal concentration of dimerizer was tested by measuring cell death in a dose range of dimerizer. 
Figure 24. Optimal time point to measure dimerizer-induced cell death was determined.

Figure 25. Dimerizer treatment induced apoptosis in FV-hCasp8 cells. 64

Figure 26. Dimerizer treatment induced necroptosis in mRIPK3-FV cells.

Figure 27. A co-culture system was estabilshed to study the effect of dying cells on

peritoneal macrophages.

Figure 28. IL-6 is increased on mRNA and protein level in necroptotic cells and in macrophages co-cultured with necroptotic cells.

Figure 29. Co-culture of macrophages and apoptotic cells led to elevated levels of

CXCL5 in the supernatant.

Figure 30. mRNA level of four different macrophage polarization marker were changed after co-culture with dying NIH 3 T3 cells.

Figure 31. The treatment procedure before RNA sequencing is depicted. 70

Figure 32. PCA plots demonstrated clear separation of treatment groups after RNA sequencing. 70

Figure 33. MA plots show the log fold change relative to mean expression. 71

Figure 34. Heat maps and hierarchical clustering of normalized counts with base mean $>30$ and $p$-value $<0.05$ was performed using Morpheus.

Figure 35. In macrophages co-cultured with apoptotic cells compared to control gene sets of cell cycle and replication were enriched after GSEA. 73

Figure 36. In macrophages co-cultured with necroptotic cells compared to control gene sets of hypoxia and the HIF1 pathway were enriched. 74

Figure 37. Gene sets enriched in macrophages co-cultured with necroptotic cells compared to macrophages co-cultured with apoptotic cells are shown.

Figure 38. Gene ontology analysis using GOrilla confirmed results of the GSEA analysis.

Figure 39. Co-culture with apoptotic cells leads to proliferation of primary peritoneal macrophages.

Figure 40. Images of macrophages before and after co-cultivation with apoptotic cells.

Figure 41. Co-culture with apoptotic cells leads to proliferation of IC-21 cells.

Figure 42. The oxygen consumption rate can be measured by a Cell Mito Stress test. . 80

Figure 43. Maximal mitochondrial respiration in macrophages co-cultured with necroptotic cells is significantly reduced. 
Figure 44. CXCL1 and CXCL13 levels were significantly regualted in macrophages cocultured with necroptotic cells 82

Figure 45. The time line of Zymosan-A-induced peritonitis is depicted. 83

Figure 46. Treatment scheme of in vivo experiments is shown. 84

Figure 47. Cell death of FV-hCasp8 and mRIPK3-FV cells was confirmed before injection into mice. 84

Figure 48. Weight loss of mice was monitored daily.

Figure 49. Death of cells in the lavage was analyzed at day 1, 3 and 6 via Annexin PI staining.

Figure 50. t-SNE maps of control animals show immune cell profiles during ZymosanA-induced peritonitis.

Figure 51. t-SNE maps of animals treated with cell death inhibitors show immune cell profiles during Zymosan-A-induced peritonitis at day 1

Figure 52. t-SNE maps of animals treated with cell death inhibitors show immune cell profiles during Zymosan-A-induced peritonitis at day 3.

Figure 53. t-SNE maps of animals treated with cell death inhibitors show immune cell profiles during Zymosan-A-induced peritonitis at day 6

Figure 54. Injection of cell death inhibitors led to changes in immune cell levels

Figure 55. t-SNE maps of animals treated with dying cells show immune cell profiles during Zymosan-A-induced peritonitis.

Figure 56. Injection of dying cells led to changes in immune cell levels

Figure 57. Concentrations of different cytokines in the lavage of mice treated with cell death inhibitors were analyzed.

Figure 58. Concentrations of different chemokines in the lavage of mice treated with cell death inhibitors were analyzed.

Figure 59. Levels of CXCL1 in the lavage of mice treated with cell death inhibitors were analyzed.

Figure 60. mRNA level of cell cycle genes in macrophages at day 1 of Zymosan-Ainduced peritonitis and zVAD.fmk injection.

Figure 61. MLKL expression is regulated by IRF1 and STAT1

Figure 62. Apoptotic cells induce proliferation of macrophages, whereas necroptotic cells influence the recruitment of neutrophils. 


\section{List of tables}

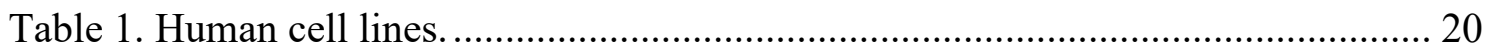

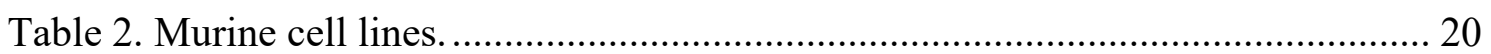

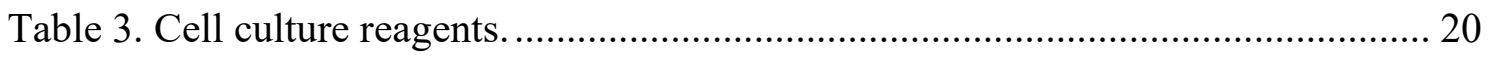

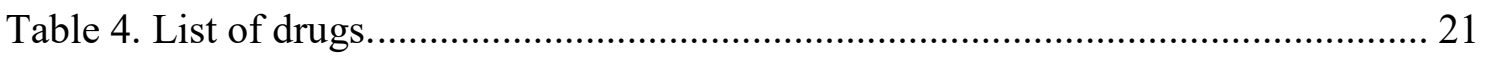

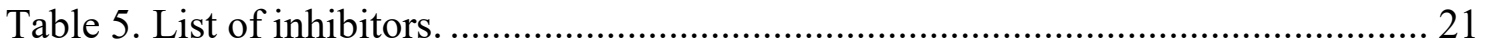

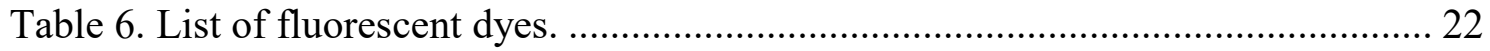

Table 7. Primary antibodies for Western Blotting....................................................... 22

Table 8. Secondary antibodies for Western Blotting.................................................. 23

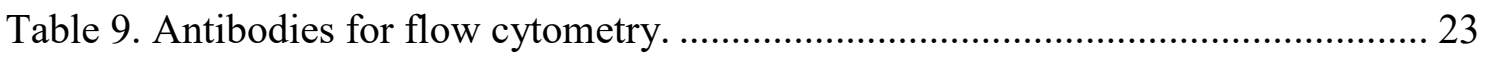

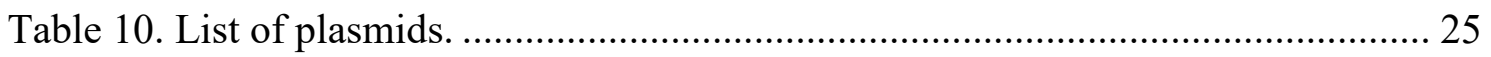

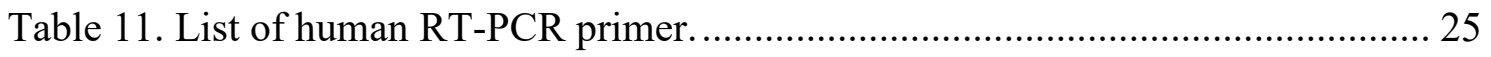

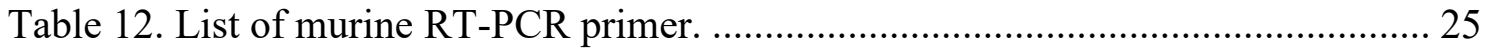

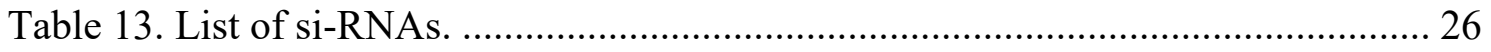

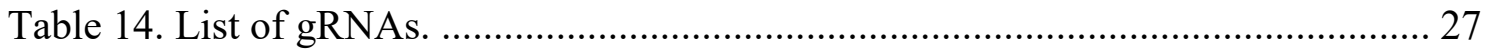

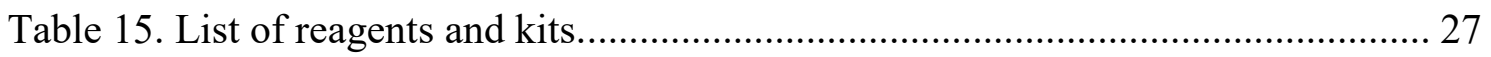

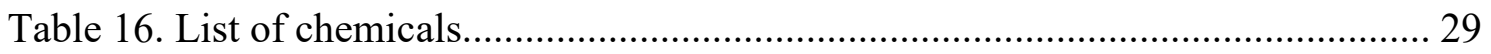

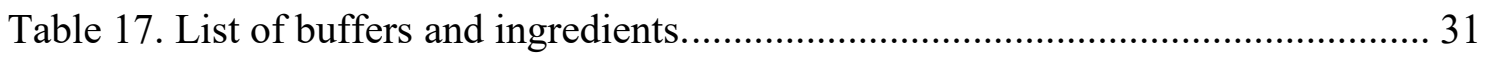

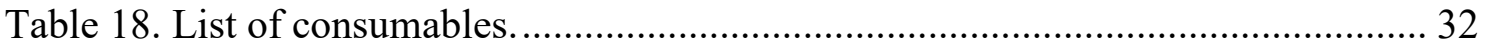

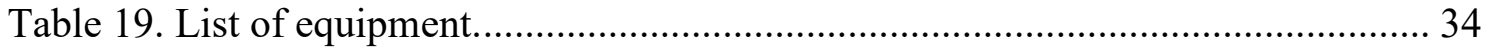

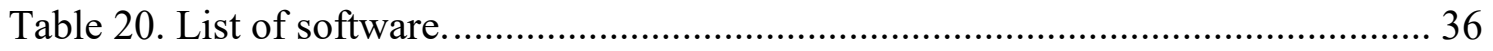

Table 21. Concentrations or densities used to seed cell lines....................................... 38

Table 22. Dimerizer-induced cell death and GFP fluorencence intensity of NIH 3T3 single clones were analyzed.

Table 23. Results for upregulation of MLKL in DNA microarrays using the platform Interferome. 


\section{Abstract}

Inducing cell death in tumor cells is a major goal of anti-cancer therapy. However, the preferable mode of cell death to induce is under debate. Apoptosis is known to be an antiinflammatory and pro-resolving type of programmed cell death, whereas necroptosis results in the release of danger-associated molecular patterns (DAMPs) and is proinflammatory. Efferocytosis of apoptotic cells by macrophages results in a pro-resolving switch of macrophages polarization and is required to induce resolution of inflammation. This impact of apoptotic cells on macrophages is a non-desired consequence of cell death in tumors, which are often characterized by an overshooting wound healing response. Moreover, apoptosis resistance is frequently observed in cancer cells. To overcome apoptosis resistance in cancer cells, necroptosis can be induced as an alternative mechanism for cancer treatment. Interferons (IFNs) play an important role in tumor immune responses and act by inducing the expression of IFN-stiumlated genes (ISGs). Furthermore, IFNs were shown to be able to induce necroptosis together with Smacmimetics when caspases are inhibited in different cancer cell lines. Necroptosis is induced by phosphorylation and activation of receptor-interacting serine/threonine-protein kinase 1 (RIPK1), RIPK3 and pseudokinase mixed lineage kinase domain-like (MLKL).

In my thesis, we first identified MLKL as an ISG in various cancer cell lines. MLKL upregulation was found to be a general feature of IFN signaling since both type I and type II IFNs increase the expression of MLKL. IFN $\gamma$ was able to upregulate MLKL at messenger ribonucleic acid (mRNA) and protein level indicating that MLKL is elevated transcriptionally. Indeed, Actinomycin D chase experiments showed that inhibition of transcription abolished MLKL upregulation upon IFN $\gamma$ treatment. Both, knockdown of the IFN $\gamma$-activated transcription factors interferon regulatory factor 1 (IRF1) and signal transducer and activator of transcription 1 (STAT1) as well as knockout of IRF1 significantly dampened MLKL mRNA upregulation, demonstrating that STAT1 and especially IRF1 are necessary to induce MLKL expression. This first part of the study highlights the upregulation of MLKL by IFN $\gamma$ as valuable tool to sensitize cells towards necroptosis and by that overcome apoptosis resistance in cancers.

When compared to apoptosis, the immune response to necroptotic cells and the polarization of macrophages phagocytosing necroptotic cells is not well studied. In most 
studies, cell death was induced by biological or chemical compounds, which may lead to artifacts by affecting the macrophages and triggering of unrelated signaling pathways. Therefore, in the second part of my thesis we used a pure cell death system of NIH 3T3 cells expressing either dimerizable caspase 8 or oligomerizable RIPK3 to induce cell death. Addition of B/B-Homodimerizer (dimerizer) to the cells resulted in apoptosis or necroptosis, which was confirmed by caspase 3/7 activation, phosphorylation of MLKL and inhibitor experiments, respectively. We analyzed the effect of dying cells on peritoneal macrophages by establishing a co-culture in a transwell system. The genetic profile of macrophages co-cultured with dying cells was evaluated by whole transcriptome RNA sequencing. In macrophages co-cultured with necroptotic cells genes corresponding to chemotaxis and hypoxia pathways were upregulated. A significant proportion of hypoxia-related pathways are mediated by hypoxia-inducible factor 1-alpha (HIF-1 $\alpha$ ), which also induces metabolic changes in polarized macrophages. We could show that macrophages co-cultured with necroptotic cells showed a decreased mitochondrial respiration, indicating an inflammatory (M1) polarization. Protein levels of chemokine C-X-C motif ligand 1 (CXCL1), which was increased in the RNA sequencing data, were also upregulated in supernatant of co-cultured macrophages and of necroptotic cells, demonstrating that necroptotic cells both secrete CXCL1 and induce gene expression of CXCL1 in peritoneal macrophages. This may influence the recruitment of neutrophils as inhibition of necroptosis during Zymosan-A-induced peritonits in mice decreased the levels of neutrophils at day 1 of this model of selfresolving inflammation.

Furthermore, RNA sequencing revealed an unexpected impact of apoptotic cells on macrophage biology as cell cycle and cell division pathways were increased. Enhanced proliferation of macrophages was confirmed by two functional assay with peritoneal macrophages isolated from mice and IC-21 macrophages. Inhibition of apoptosis during Zymosan-A-induced peritonits in mice demonstrated decreased mRNA levels of cell cycle mediators in peritoneal macrophages. Simultaneously with cell cycle activation, gene sets of prostaglandin $\mathrm{E}_{2}\left(\mathrm{PGE}_{2}\right)$ signaling were upregulated during RNA sequencing. In the second part of my thesis we could demonstrate, that apoptotic cells induce transcription of cell cycle genes and proliferation of macrophages and necroptotic cells are able to influence the chemokine profile of macrophages and thereby the recruitment of neutrophils. 


\section{Introduction}

\subsection{Programmed cell death}

Cell death can be found in almost every multicellular life form in the animal kingdom. It is important to maintain a homeostatic balance between proliferation and death of cells as uncontrolled cellular proliferation can lead to cancer and excessive cell death can be found in diseases like Alzheimer's disease, Parkinson's disease or rheumatoid arthritis [1-3]. Several different types of programmed cell death have been described in the past including apoptosis, necroptosis, pyroptosis, autophagy-dependent cell death, ferroptosis and more. The focus in this study lies on apoptosis and necroptosis.

\subsubsection{Apoptosis}

Apoptosis is the most studied pathway of cell death and escape of apoptosis is a hallmark of cancer. This cell death pathway was first described in Caenorhabitis elegans and distinct morphological changes can be observed [3]. First, cells shrink and chromatin in the nucleus condensates, followed by membrane blebbing and fragmentation of the nucleus and in the end formation of apoptotic bodies, whereas the plasma membrane stays intact and no cytosolic material is released into the surrounding tissue $[2,4]$. Furthermore, apoptotic cells present phosphatidylserine (PS) on their outer membrane, which functions as an "Eat-me" signal for macrophages (see section 1.2.). That is why apoptosis is believed to be immunologically silent [5]. To avoid secondary necrosis and inflammation in vivo, macrophages phagocytose apoptotic cells before they expose cytosolic material [6]. Apoptosis plays an important role in development, aging and homeostasis as around one million cells undergo apoptosis every second in adult humans [2, 4, 7]. Induction of apoptosis leads always to activation of caspases. Eleven different Cysteine proteases are known in humans. Caspases cleave their substrates after Asparagin residues and are activated by proteolytic cleavage of their zymogens [2]. Two main apoptotic pathways can be distinguished: intrinsic and extrinsic apoptosis (Figure 1). 


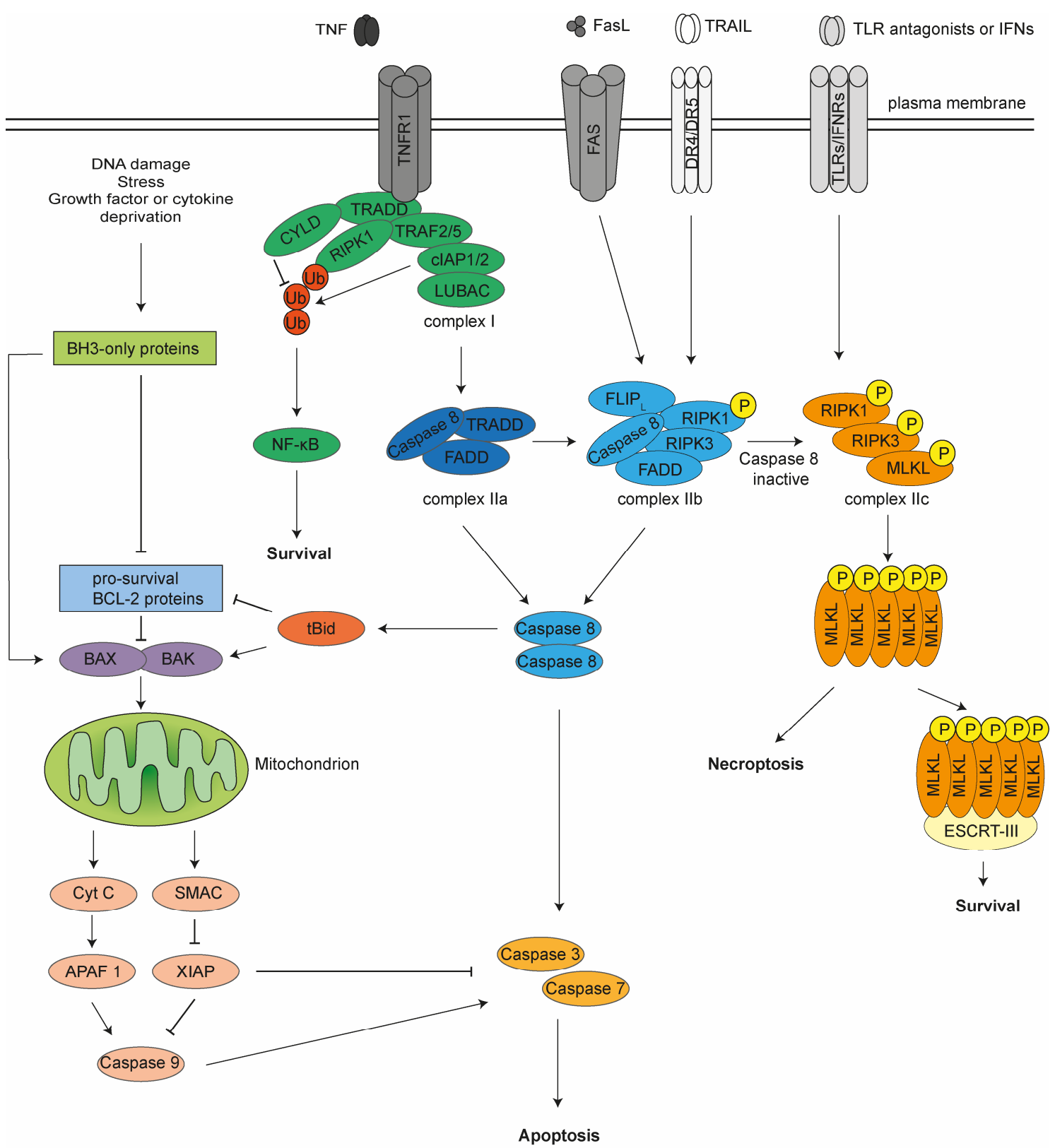

Figure 1. Programmed cell death pathways. Detailed description of intrinsic and extrinsic apoptosis and necroptosis can be found in the text below. Figure modified after $[8,9]$.

\subsubsection{Intrinsic apoptosis}

Intrinsic or mitochondrial apoptosis can be induced by positive or negative signals [1]. Positive signals, like hypoxia, radiation, toxins, reactive oxygen species (ROS), endoplasmic reticulum (ER) stress or viruses as well as negative signals, like absence of cytokines, hormones or growth factors are able to induce apoptosis by an irreversible mitochondrial outer membrane permeabilization (MOMP) [1,5]. MOMP is controlled and mediated by pro- and anti-apoptotic members of the B-cell lymphoma 2 (BCL2) protein family [5]. Bcl-2-associated $\mathrm{X}$ protein (BAX) and Bcl-2 homologous 
antagonist/killer (BAK) are transcriptionally or post-translationally activated upon the factors described above and assemle to oligomers in the mitochondrial outer membrane. This leads to MOMP and release of intermembrane proteins like cytochrome c $[5,10]$. Under normal conditions pore formation is prevented by binding of anti-apoptotic/prosurvival BCL2 family members, like BCL2, B-cell lymphoma-extra large (BCL-X $)$ or myeloid cell leukemia 1 (MCL-1) to BAX and BAK [5]. Cytochrome $\mathrm{c}$ binds to apoptotic protease activating factor 1 (APAF1) and pro-caspase 9 building the so-called apoptosome and leading to caspase 9 activation [5]. Activated initiator caspase 9 is able to induce a proteolytic cascade and catalyzes the activation of executioner caspases 3 and $7[1,5,10]$. The activation of caspases is promoted by binding of the mitochondrial protein second mitochondria-derived activator of caspases (Smac) to inhibitors of apoptosis (IAPs), which otherwise bind and inhibit caspases [1, 5, 10]. In cancer therapy, Smac-mimetics, like BV6, have been developed to induce apoptosis in cancer cells [10].

\subsubsection{Extrinsic apoptosis}

Extrinsic apoptosis is induced by binding of death ligands to surface-associated death receptors (DRs) [8]. Death ligands are produced by immune cells like natural killer (NK) cells or macrophages, which detected damaged cells $[1,5]$. DRs belong to the tumor necrosis factor (TNF) superfamily, which includes TNF receptor-1 (TNFR1), Fas receptor (FAS), TNF-related apoptosis-inducing ligand (TRAIL) receptors-1 and -2, DR3 and DR6 [1, 11].

Depending on the activated DR a different complex is formed, which activates caspase 8 . Binding of TNF to its receptor (TNFR1) leads to trimerization of the receptor and formation of complex I, which consists of TNF receptor type1-associated death domain (TRADD), TNF receptor associated factor 2 (TRAF2), TRAF5, cIAP1, cIAP2, RIPK1, cylindromatosis (CYLD) and linear ubiquitin chain assembly complex (LUBAC; consisting of haem-oxidized IRP2 ubiquitin ligase-1, HOIL-1L; HOIL-1-interacting protein, HOIP; and SHANK-associated RH domain-interacting protein, SHARPIN) [5, $8,12]$. Depending on the stress level, stimuli, cell type, disease or pharmacological agents applied, complex I can lead to cell survival, apoptosis or necroptosis [8]. Upon polyubiquitination of RIPK1 by cIAPs, nuclear factor kappa-light-chain-enhancer of activated B-cells (NF-kB) and mitogen-activated protein kinase (MAPK) pathways can be activated, which favors a pro-inflammatory signaling and survival of the cell. 
When cIAPs are inhibited (for example by Smac-mimetic) or RIPK1 is de-ubiquitinated by CYLD, RIPK1 and TRADD dissociate from complex I and complex IIa (or deathinducing signaling complex, DISC) is formed $[1,5,8]$. Two different forms of complex II are known: complex IIa and complex IIb. Complex IIa consists of TRADD, Fasassociated protein with death domain (FADD) and pro-caspase 8 and is able to induce RIPK1-independent apoptosis [8]. Complex IIb is composed of RIPK1, RIPK3, FADD, FLICE-like inhibitory protein large $\left(\right.$ FLIP $\left._{\mathrm{L}}\right)$ and caspase 8 and favours RIPK1-kinaseactivity-dependent apoptosis [12]. Caspase 8 is activated by dimerization and autoproteolytic cleavage of pro-caspase 8 monomers at the complex $[1,5]$, which leads to activation of executioner caspases 3 and 7 and cell death or cleavage of BH3 interactingdomain death agonist (BID), which as a link to intrinsic apoptosis causes mitochondrial damage and cell death [2]. At complex IIb caspase 8 is able to cleave pro-necroptotic proteins (RIPK1, RIPK3 and CYLD) to inhibit necroptosis and induce apoptosis $[1,5,8]$.

\subsubsection{Necroptosis}

Different types of regulated necrosis were described with the same morphology of organelle swelling, plasma membrane rupture, cell lysis and release of intracellular components into the evironment [8]. Necroptosis, among others like parthanatos, ferroptosis, pyroptosis and NETosis, is the best decribed pathway of regulated necrosis $[8,12]$. Extrinsic apoptosis and necroptosis start both with the ligation of DRs. As a backup mechanism for apoptosis, necroptosis is only activated when caspase 8 is inhibited or absent $[8,13]$. Caspase 8 can be inhibited for example by cFLIP or pharmacological and genetic ablations can lead to absence of caspase 8. Furthermore, caspase 8 can be repressed by a high expression of necroptotic or virally-expressed proteins [8].

Upon caspase 8 inhibition, RIPK1 is free to form complex IIc and RIPK1 and RIPK3 are activated by auto- and trans-phosphorylation. This RIP homotypic interaction motif (RHIM)-depentend oligomerization and phosphorylation of RIPK3 (human RIPK3 at Ser227 or mouse RIPK3 at Ser232) leads to recruitment and phosphorylation of MLKL (human MLKL at Thr357 and Ser358 or mouse MLKL at Ser345, Ser347, Ser352 and Thr349) $[8,12,13]$. Phoshorylated MLKL is able to oligomerize and translocate to the plasma membrane, which leads to influx of calcium ions, ROS production and rupture of the plasma membrane $[8,13]$. The deatiled mechanism how MLKL is able to execute 
necroptosis are not fully understood yet. MLKL may be able to form pores itself in the plasma membrane or interacts with and activates ion channels [14-19]. The following death of the cell by necroptosis can be delayed by binding of endosomal sorting complexes required for transport III (ESCRT-III) to MLKL oligomers at the plasma membrane $[8,20]$. Due to the release of intracellular material into the environment necroptosis is an inflammatory cell death pathway [8].

Additionally to TNFR1, FAS and DR4/5, necroptosis can be induced by ligation of tolllike receptors (TLRs) or interferon receptors (IFNRs) [8]. Binding of IFNs to IFNR results in the activation of the Janus kinase (JAK)/STAT pathway and in the end activation of complex IIc in a transcription-dependent pathway [12, 21].

\subsubsection{Dimerizer-induced cell death}

Chemical induction of proximity of proteins is an established method to activate one specific biological process in cells without side-effects of the inducer [22]. These inducible protein interactions have provided fundamental insight into many biological signaling pathways including apoptosis and necroptosis. The chemical induction is based on the binding of ligands (FK-506 or Rapamycin) to FK-506 binding proteins (FKBPs). FKBPs are cis-trans peptidyl-prolyl isomerases (PPIase), belong to the immunophilin family and are expressed in all eukaryotes [23]. Binding of FK-506 or Rapamycin leads to immunosuppressive effects and therefore these drugs are used as therpeutic agents to stop allograft rejection in post-transplantation patients [23]. The chemical induction of proximity can be used as a tool to induce dimerization or oligomerization of targeted proteins by fusing the protein of interest to one or more modified FKBP domains. Symmetrical dimerization can be achieved by treatment with a bivalent ligand like synthetic dimers of FK-506 or Rapamycin [23]. These ligands are specific for modified FKBP domains e.g. FKBP F36V mutant (FV) and do not bind to endogenous FKBP. Andrew Oberst and colleagues developed inducible, pure cell death systems of apoptosis and necroptosis. They showed that apoptosis can be induced by caspase 8 dimerization and necroptosis by RIPK3 oligomerization [24, 25]. In their system, the death effector proteins caspase 8 and RIPK3 were fused to FV domains. Dimerization was induced by AP20187 [26]. AP20187 (B/B Homodimerizer, dimerizer) consists of two derivate molecules of FK-506, which are chemically linked (Figure 2). This bivalent ligand is 
selevtive for FV and has increased solubility in aqueous solutions compared to other derivates of FK-506 [23].<smiles>CC[C@H](C(=O)N1CCCC[C@H]1C(=O)O[C@H](CCc1ccc(OC)c(OC)c1)c1cccc(OCC(=O)NCC(CNC(=O)COc2cccc([C@@H](CCc3ccc(OC)c(OC)c3)OC(=O)[C@@H]3CCCCN3C(=O)[C@@H](CC)c3cc(OC)c(OC)c(OC)c3)c2)CNC(=O)CC(C)C)c1)c1cc(OC)c(OC)c(OC)c1</smiles>

Figure 2. Chemical structure of the homodimerizer AP20187 (B/B homodimerizer). Adapted from [23].

\subsection{Clearance of dead cells}

Removal of dying and dead cells is not only necessary to prevent release of proinflammatory cellular content like DAMPs and thereby the induction of chronic inflammation and autoimmune disease, but contributes also to tissue homeostasis [27, 28]. Dead cells are removed by either professional phagocytes like macrophages and dendritic cells or neigbouring non-professional phagocytes. The process of phagocytosis of dead cells is called efferocytosis and several factors are needed for its success [7, 29]: Dead cells have to release "Find-me" signals, express "Eat-me" signals on their surface and decrease the expression of "Don't-eat-me" signals (Figure 3). Apoptotic cells release "Find-me" signals induced by caspase activation such as membrane lipids (lysophosphatidylcholine, LPC and sphingosine-1-phosphate, S1P), nucleotids (Adenosine triphosphate, ATP and Uridine-triphosphate, UTP) and chemokines (CX3CL1, Fractalkine) to recruit phagocytes [7, 27-29].

Clearance of necrotic or necroptotic cells is not studied completely but these cells or cells in their environment release "Find-me" signals, too. DAMPs like deoxyribonucleic acid (DNA), RNA, high mobility group box 1 (HMGB1), histones, actin, calcium-binding S100 proteins, heat-shock proteins, ATP and uric acid can be released by necroptotic cells 
as well as formyl-peptides, chemokines or leukotriene B4 (LTB4), which are powerful chemoattractants to leukocytes [30].
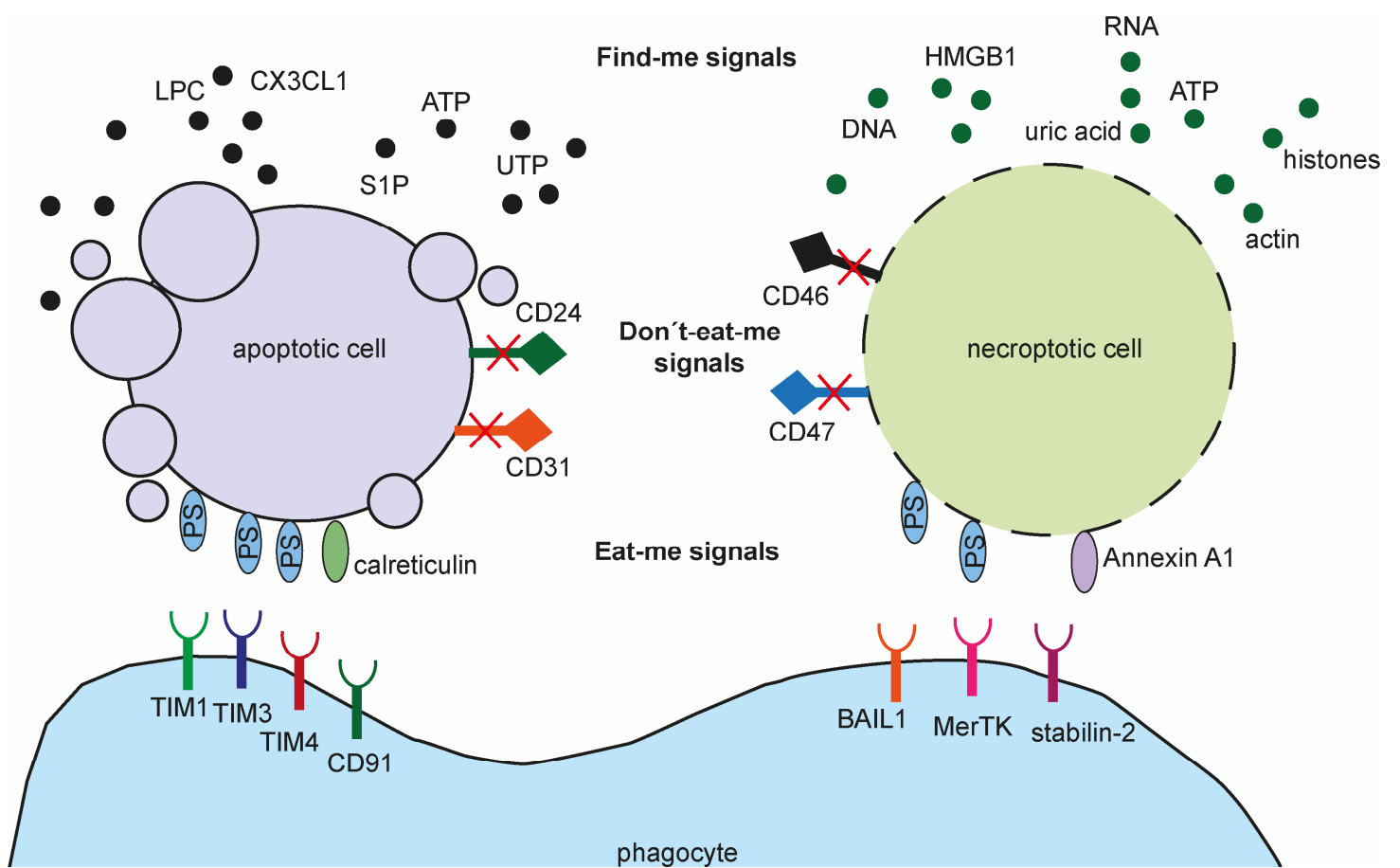

Figure 3. Factors mediating clearance of dying cells. Figure modified after [27, 30].

To engulf apoptotic cells they have to display "Eat-me" signals on their surface. The most prominent "Eat-me" signal of apoptotic cells is PS [27]. In the plasma membrane of living cells, PS is exclusively found in the inner leaflet. Upon activation of caspase 3 , the flippase ATP11 is cleaved and inactivated and the scramblase XKR8 is activated, which results in exposure of PS on the surface of apoptotic cells [7, 27]. The exposure of PS is not limited to apoptotic cells and was also seen on necroptotic cells. Here, activation of MLKL during necroptosis leads to the loss of phospholipid asymmetry [27]. As the mechanisms of PS exposure differ between apoptotic and necroptotic cells, this may be the reason why apoptotic and necroptotic cells are cleared with different efficacies [30]. PS is recognized by phagocytes through the surface receptors T-cell immunoglobulin and mucin-domain containing-1 (TIM1), TIM3 and TIM4, Bail1, tyrosine-protein kinase Mer (MerTK), stabilin-2 and members of the CD300 family [7, 27, 30]. Moreover, bridging molecules like MFG-E8, Del-1, Gas6, protein S and C1q are able to mediate the binding of PS to phagocytes $[28,30]$. Binding of PS by surface receptors results in secretion of anti-inflammatory cytokines interleukin 10 (IL-10), transforming growth factor beta (TGF- $\beta$ ), and prostaglandins and inhibition of the production of pro-inflammatory 
cytokines TNF $\alpha$ and IL-1 $\beta$ [27]. Other "Eat-me" signals of apoptotic cells are LPC and calreticulin [7, 27, 28]. Further "Eat-me" signals of necrotic or necroptotic cells are Annexin A1 and factors of the complement system. Uptake of necrotic cells by Annexin A1 recognition is able to dampen the secretion of pro-inflammatory cytokines, which indicates that necrosis can have anti-inflammatory effects [30].

To be effectively cleared, dying cells have to lose "Don't eat me" signals such as CD24, CD31, CD46 and CD47 [7, 27, 28]. Another reason why necroptotic cells are cleared less efficiently than apoptotic cells may be that they do not downregulate "Don't eat me" signals and show increased surface expression of CD47 [30]. In summary, dying cells must present a sufficient amount of "Eat-me" signals and decrease the expression of "Don't-eat-me" signals on their surface in order to induce their removal by phagocytes [7].

Upon recognition of dying cells, reorganization of the plasma membrane and actin remodeling are required to engulf the cell and form the phagosome. Phagosomes fuse with lysosomes and the cargo of phagosomes is digested by proteases, nucleases and lipases. Expression of the transcription factor peroxisome proliferator-activated receptor gamma (PPAR $\gamma$ ) is increased in response to phagocytosis of apoptotic cells and enhances efferocytosis [27]. Furthermore, macrophages which phagocytosed apoptotic cells change their polarization towards a pro-resolving phenotype $[28,29]$. This switch of polarization is described further in section 1.3.3.

As many autoimmune and inflammatory diseases, like systemic lupus erythematosus, multiple sclerosis, Alzheimer's disease, Parkinson's disease, Huntington's disease, atherosclerosis and more, are associated with defective clearance of dead cells, it will be of great benefit to gain new insights into the regulation of efferocytosis and develop new treatment strategies [27]. One possibility will be the administration of apoptotic cells as therapy together with stimulation of efferocytosis. It was shown before that apoptotic cells are able to control inflammatory or autoimmune disorders in experimental models by inducting resolution of inflammation [29]. Moreover, apoptotic cells are an already established treatment for cutaneous T cell lymphoma in humans [7]. 


\subsection{Inflammation}

Inflammation is a biological process, which is necessary to eliminate bacterial or viral infections, e.g. after injuries, and promotes tissue repair as well as establishing an immune memory [31-33]. The five cardinal signs of inflammation were described by Celsus and Virchow and are color (heat), dolor (pain), rubor (redness), tumor (swelling) and functio laesa (loss of tissue function) [32]. An uncontrolled and unresolved inflammation may become chronic due to prolonged exposure to inflammatory stimuli or an inappropriate reaction to self-molecules and may lead to diseases like rheumatoid arthritis, systemic lupus erythematosus, chronic granulomatous disease, asthma, atherosclerosis, autoimmune disease, diabetes and cancer $[31,32,34]$.

\subsubsection{Onset of inflammation}

During the onset of inflammation, pathogens, infected cells and cells dying by necroptosis or pyroptosis release DAMPs or pathogen-associated molecular patterns (PAMPs), which are recognized by pattern recognition receptors (PRRs) on resident cells [35]. This results in the production of pro-inflammatory mediators like cytokines and chemokines, which are able to induce pro-inflammatory signaling pathways [31,32]. Next, granulocytes like neutrophils and mononuclear phagocytes are recruited from the blood by upregulation of cell adhesion molecules on circulating leukocytes and endothelial cells [31]. Monocytes and macrophages are recruited to the site of inflammation through the action of chemokines like chemokine (C-C motif) ligand 2 (CCL2), IL-8 and CXCL-8. Neutrophils are able to destroy, phagocytose and thereby eliminate microorganisms through production of ROS and formation of neutrophil extracellular traps, which is a prerequisite to proceed towards resolution of inflammation [31, 32] (Figure 4). 


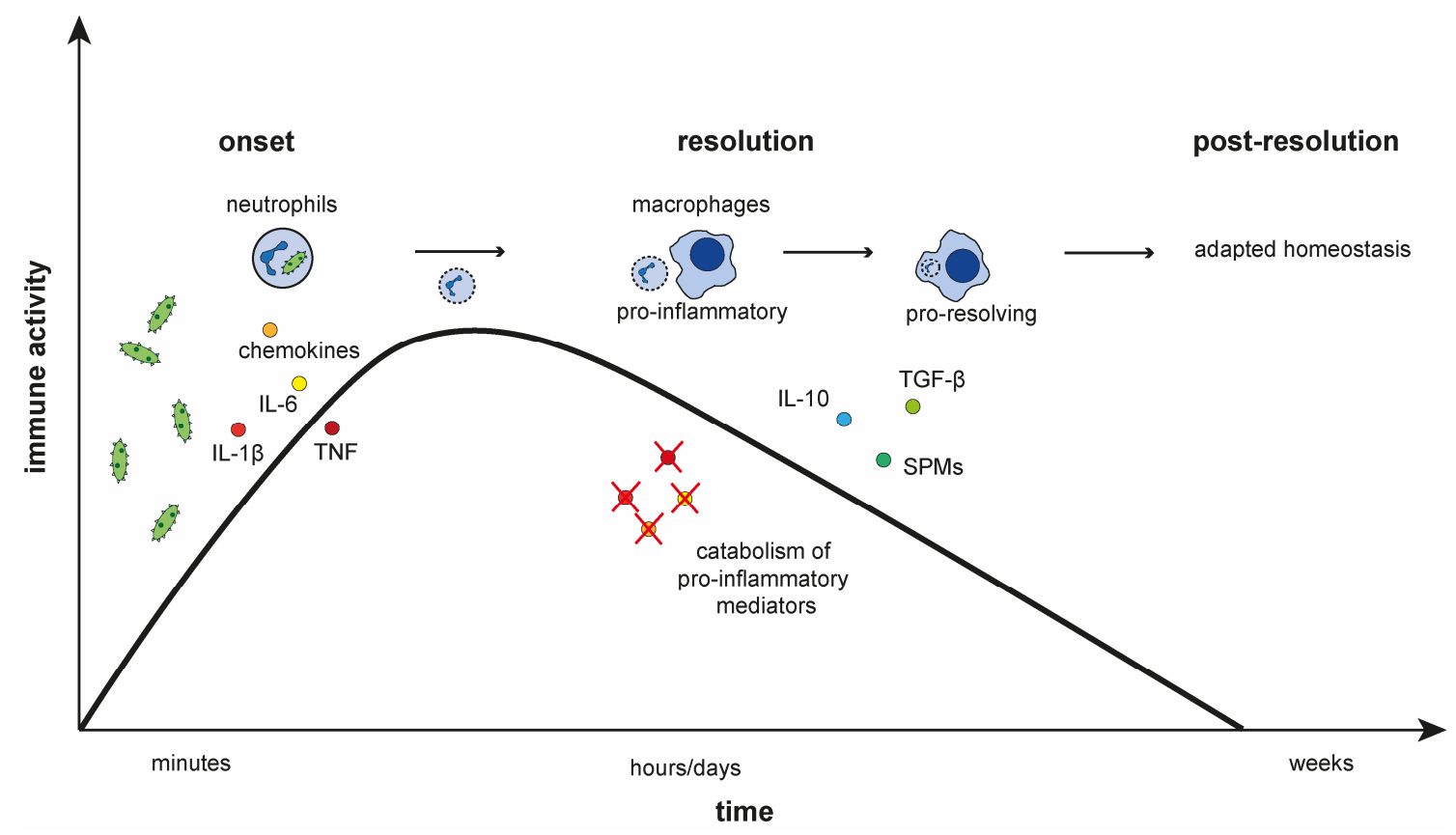

Figure 4. Time line of inflammation and its resolution. Figure modified after [31, 36].

\subsubsection{Resolution of inflammation}

Resolution of inflammation is necessary to prevent the development of chronic inflammations and induce restoration of homeostasis. Historically it was considered a passive process but is now proven to be an active and regulated pathway [31, 32]. Resolution of inflammation consists of several steps: elimination of inducing stimulus, dampening of pro-inflammatory signals, catabolism of pro-inflammatory mediators, elimination of inflammatory cells and efferocytosis of apoptotic neutrophils [31, 32].

After elimination of inducing stimulus like bacteria or viruses by neutrophils as described above, production of pro-inflammatory signals has to be stopped. Several different mediators like e.g. RP105, CYLD, $\mathrm{PGE}_{2}$ and microRNAs or regulation of mRNA levels of pro-inflammatory cytokines are able to reduce production of pro-inflammatory signals. Next, pro-inflammatory mediators are removed by e.g. catabolizing of prostaglandins or binding of chemokines without initiation of signaling pathways. Furthermore, inflammatory cells have to leave the site of inflammation. They can migrate back into the systemic circulation, exit the tissue through lymphatic drainage or undergo cell death. This step is tightly regulated but can also be disturbed. For example, soluble mediators including IL-6, IL-8, granulocyte-macrophage colony-stimulating factor (GM-CSF) and bacterial products can prolong the survival of e.g. granulocytes. 
Apoptotic cells are phagocytosed by resident macrophages or monocyte-derived macrophages (MDMs), which results in changes in the polarization of the macrophages from a pro-inflammatory to a pro-resolution phenotype (further described in section 1.4.). Pro-resolution macrophages are decribed to be the main producer of specialized proresolving mediators (SPMs) like resolvins, protectins and maresins, which contribute to resolution of inflammation.

Once macrophages have left the site of inflammation through lymphatic-drainage or local apoptosis, the resolution of the inflammation is complete. Recently, an alternative model was suggested, in which resolution is not the end of the immune response. It was shown that resolution results in a state of adapted homeostasis, where a second influx of leukocytes occurs, which persits longer in the tissue and helps to connect innate and adaptive immune systems [31, 32].

As an anti-inflammatory therapy, influx of neutrophils is often targeted by non-steroidal anti-inflammatory drugs (NSAIDs), anti-inflammatory recombinant cytokines or neutralizing blocking antibodies $[31,32]$. Inducing resolution of inflammation alone or in combination with anti-inflammatory drugs could be more successful [32]. As apoptosis of inflammatory cells results in resolution of inflammation, inducing of apoptosis or application of apoptotic cells could be a good target for treatment of inflammations (see also section 1.2.).

\subsection{Macrophage polarization}

Macrophages are part of the innate immune system and are present in every tissue. They have several important functions such as phagocytosis of pathogens, infected or dead cells and presentation of antigens. In response to different environmental stimuli, macrophages are able to express distinct proteins and cytokines, which is called activation or polarization of macrophages [37]. This shows the high plasticity of macrophages, with the notion that polarization can also be re-programmed. Historically, two different polarization states of macrophages with different functions are distinguished: Classically activated or pro-inflammatory M1 macrophages and alternatively or anti-inflammatory M2 macrophages [37]. Whereas M1 macrophage polarization is induced by IFN $\gamma$, TNF $\alpha$ or LPS, polarization of M2 macrophages is induced by IL-4, IL-10 or IL-13. M1 macrophages produce higher levels of pro-inflammatory cytokines (TNF- $\alpha$, IL-1 $\alpha$, IL-1 $\beta$, IL-6, IL-12, IL-23) and chemokines (CXCL9, CXCL10, CXCL11) and are able to remove 
pathogens by ROS and nitric oxide (NO) production [29, 37, 38]. M2 macrophages show a potent phagocytosis phenotype, have an anti-inflammatory cytokine profile (expression of IL-10 and TGF- $\beta$ ) and recruit T helper cells, regulatory T cells, eosinophils and basophils by release of CCL17, CCL18, CCL22 and CCL24 [37]. M2 macrophages help to dampen inflammations, promote tissue remodeling and immunoregulation. Furthermore, they contribute to resolution of inflammation by production of lipid mediators like SPMs (resolvins, maresins and protectins) [35]. Generation of NO in M2 macrophages is inhibited by enhanced expression of arginase-1 and ROS production is reduced by activation of PPAR $\gamma$ [39]. The metabolism of macrophages changes also during polarization. M1 macrophages show an enhanced glycolysis by stabilization of the transcription factor HIF-1 $\alpha$ and reduced mitochondrial activity. M2 macrophages switch to high mitochondrial activity and arginine metabolism [38]. As described above, efferocytosis of apoptotic cells activates macrophages and leads to an anti-inflammatory M2 polarization [29, 39]. Nevertheless, the M1/M2 classification of macrophages does not reflect all different subsets of macrophages, especially in vivo, where macrophages exist in a spectrum of populations depending on their microenvironment in the tissue [ 35 , $37,38]$.

\subsection{Proliferation of macrophages and activation of the cell cycle}

Macrophages originate from monocytes, which are produced in the bone marrow, enter the peripheral blood and differentiate into macrophages in the tissue. This historical view has changed since macrophages were found to derive also from the yolk sac during embryonic development and have self-renewal capacities [35, 37]. The loss of macrophages during inflammation can be compensated by local proliferation and additionally in diseases like atherosclerosis, kidney disease, acetaminophen-induced liver failure, and chronic lung inflammation proliferation of macrophages was observed [40]. Macrophage proliferation can be initiated by extrinsic factors like macrophage colonystimulating factor (M-CSF), GM-CSF, and IL-4, and intrinsic factors such as transcription factors [40]. To proliferate, cells have to enter the cell cycle, which can be divided into four phases: Gap $1\left(\mathrm{G}_{1}\right)$, synthetic $(\mathrm{S}), \mathrm{G}_{2}$ and mitotic $(\mathrm{M})$ phase [41] (Figure 5). 


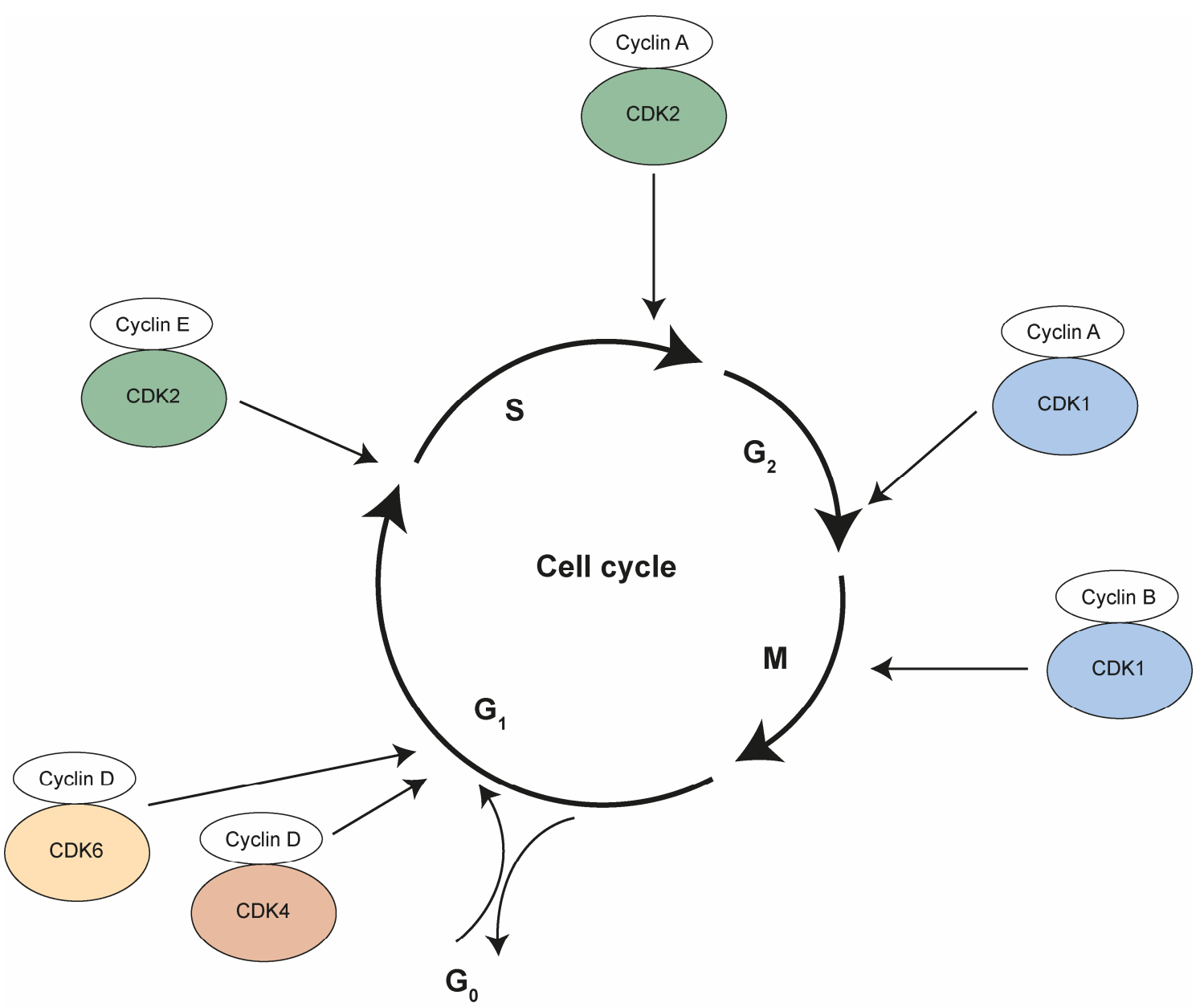

Figure 5. Cell cycle progression. Figure modified after [42].

Most of the non-growing cells in the mammalian body are in the resting $\mathrm{G}_{0}$ phase as they are not proliferating and exit the cell cycle. The cell cycle is tightly regulated by serine/theronine protein kinases called cyclin-dependent kinases (CDKs) and cyclin proteins $[7,41]$. CDKs are activated by phosphorylation and cyclin binding at specific points in the cell cycle and activate cell cycle processes by phosphorylation of proteins. Most cyclins are expressed and degradated periodically during the cell cycle. Further regulators of the cell cycle are CDK inhibitors, DNA damage checkpoints and spindle checkpoints. During $\mathrm{G}_{1}$ phase, the cell prepares DNA synthesis by production of proteins, RNA and membrane components [7]. Entry into $\mathrm{G}_{1}$ is regulated by CDK4 or 6-cyclin D complexes, whereas progression from $\mathrm{G}_{1}$ to $\mathrm{S}$ phase is mediated by CDK2-cyclin $\mathrm{E}$ interaction [42]. The complex of cyclin $\mathrm{A}$ and $\mathrm{CDK} 2$ is required during $\mathrm{S}$ phase, in which DNA is replicated. After the $S$ phase cells are growing again in the $\mathrm{G}_{2}$ phase to prepare for mitosis [42]. The complex of CDK1 with cyclin A promotes the entry into mitosis in the late $\mathrm{G}_{2}$ phase. Mitosis is then regulated by interaction of CDK1 with cyclin B [42]. 
During mitosis (M phase) DNA is divided equally into two daughter cells. This phase can be divided into prophase, metaphase, anaphase and telophase [7].

\subsection{Interferon signaling}

Interferons (IFNs) are cytokines that are produced by immune cells upon virus infections and are able to induce expression of IFN-stimulated genes (ISGs) [43]. One study even described $10 \%$ of the human genome are ISGs [44]. IFNs are subdivided into three groups: type I (IFN $\alpha, \beta, \varepsilon, \kappa$ and $\varpi)$, type II (IFN $\gamma$ ) and type III (IFN $\lambda 1, \lambda 2, \lambda 3$ and $\lambda 4$ ) IFNs [43]. Type I IFNs are released shortly after recognition of PAMPs by PRRs [45, 46]. Type II IFN is mainly produced by NK cells during viral infections and is able to enhance the adaptive antiviral response. Furthermore, IFN $\gamma$ induces M1 polarization of macrophages and inhibits M2 polarization [45]. Upon binding of IFNs to their receptors (type I: IFNAR1/IFNAR2, type II: IFNGR1/IFNGR2 and type III: IFNLR1/IL10R2) the JAK/STAT pathway is activated (Figure 6) [43, 45]. As type III IFNs are not studied in this thesis, we will focus on type I and II IFN-induced pathways. 


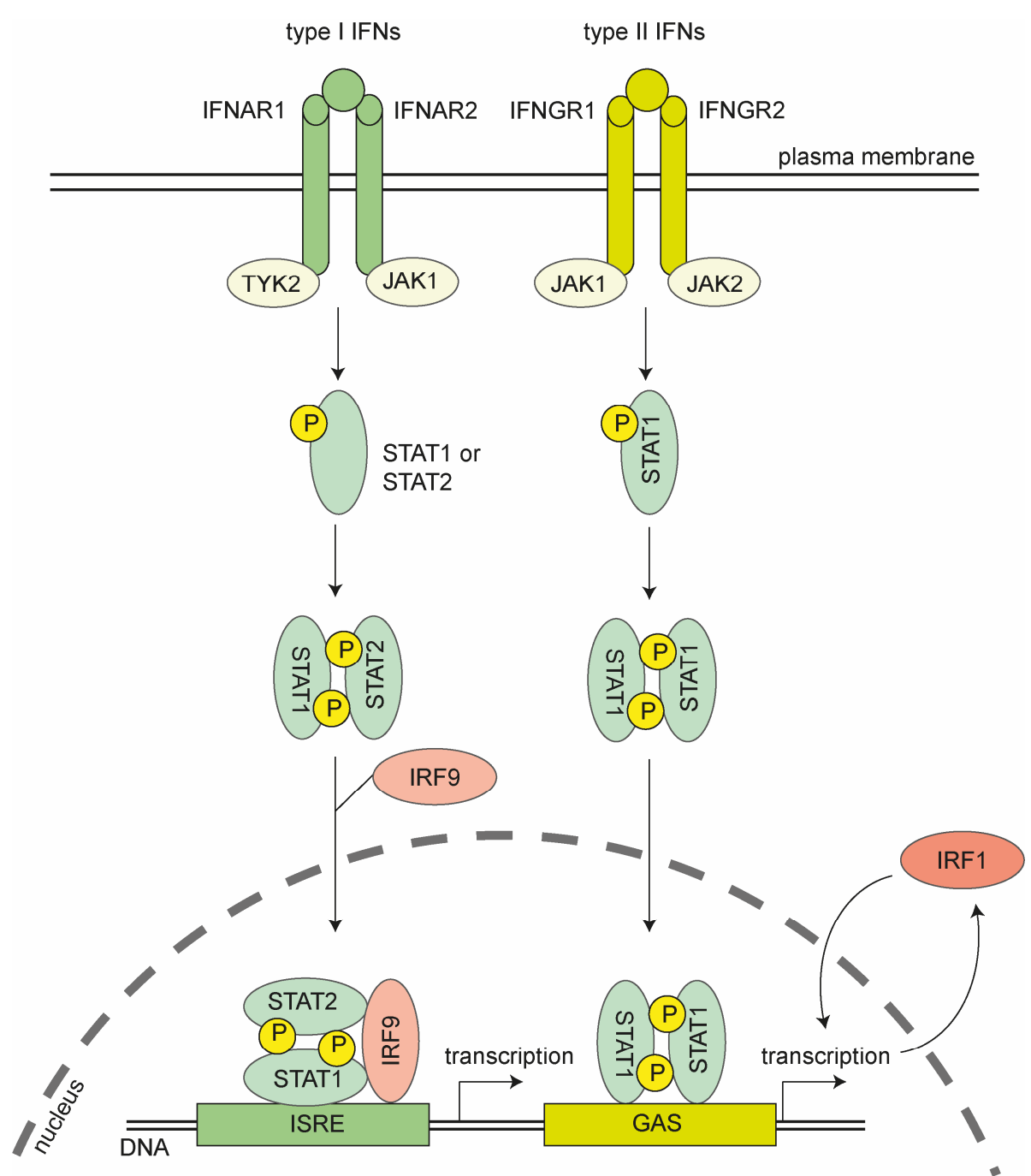

Figure 6. IFN-induced signaling pathways. The detailed mechanisms of type I and type II IFN signaling are described below. Figure modified after [43, 47].

The IFNR subunits bind different members of the Janus activated kinase (JAK) familiy (IFNAR1 interacts with tyrosine kinase 2 (TYK2), IFNAR2 and IFNGR1 with JAK1 and IFNGR2 with JAK2), which are activated by autophosphorylation. Afterwards, STATs are phosphorylated and activated by JAKs and form homo- or heterodimers [47]. Whereas type I IFNs induce activation of the transcription factor complex ISG factor 3 (ISGF3) consisting of phosphorylated STAT1 (pSTAT1), pSTAT2 and IRF9, type II IFN leads to phosphorylation and homodimerization of STAT1 and formation of IFN $\gamma$-activated factor (GAF) [43, 47]. These activated complexes bind either IFN-stimulated response elements (IRE) or gamma-activated sequences (GAS) in upstream promoter regions of ISGs and induce their transcription [43]. ISGs play a role in antiviral defense, stimulation of adaptive immunity and have growth-inhibitory effects [43, 45, 47]. Some of the ISGs like IRF1 and IRF7 are able to induce ISGs themselves and are also targets of other pathways 
like NF-kB or IL-1 signaling [43]. Moreover, expression of many genes is downregulated upon IFN stimulation, for example type I IFNs are able to induce antiproliferative elements and suppress innate immune cell function [43, 45].

IRF1 is a short-lived protein induced by IFN or NF-kB stimulation but is also expressed at low levels at basal conditions, where it can be found in the nucleus [46]. This basal expression is necesarry for the constitutive defense against viruses. IRF1 consists of three functional domains: N-terminal DNA binding domain (DBD), nuclear localization signal (NLS) and a C-terminal IRF-association domain (IAD2). To activate the evolutionary conserved transcriptional activity of IRF1, it binds IRF-binding elements (IRF-R) containing GAAA sequences at the promoter. IRF1 expression is more potently induced by type II IFN as the promoter has a single GAS element. Through its IAD2 domain IRF1 can interact with other transcription factors, for example with STAT to induce expression of ISGs. Thereby, IRF1 expression is induced by IFNs in a positive feedback loop (Figure 2) $[46]$.

Cytokines like IFNs have been studied as anti-cancer drugs for several years [48, 49]. Their ability to activate the immune system, which is able to recognize and destroy cancer cells, has turned them into potential cancer drugs. Indeed, preclinical experiments in murine models of cancer showed benefical effects. IFN $\alpha$ was the first cytokine approved for treatment of cancer (hairy cell leukemia in 1986) [48]. Meanwhile, IFN $\alpha$ is approved for several other cancer types (as adjuvant treatment for patients with high-risk melanoma, as first-line treatment for patients with AIDS-related Kaposi's sarcoma, follicular lymphoma, chronic myelogenous leukemia, condyloma acuminata, and cervical intraperitoneal neoplasms) [48]. IFN $\gamma$ is not approved as cancer drug yet, but showed promising effects in preclinical studies. Treatment of cancer with IFNs is a double-edged sword as IFNs are able to induce immune reactions but are also able to induce production of inhibitory mediators [49]. Therefore, the high expectations of IFNs as cancer treatment have not been fullfilled yet. With the development of for example, antibody-IFN fusion proteins, researchers want to overcome the disadvantages of IFNs [48]. 


\section{Aims of the study}

Necroptosis is a promising cell death pathway to overcome apoptosis resistance in cancers. It was shown before that IFNs are able to induce necroptosis by activation of the JAK-STAT signaling pathway and that IRF1 contributes to Smac-mimetic/IFN $\gamma$-induced necroptosis in cancer cell lines. Upregulation of MLKL in IFN $\gamma$ treated cells was seen as a secondary finding. Therefore, the aim of the first part of this study was to analyze the IFN-induced upregulation of MLKL expression in cancer cells to better understand how IFNs regulate necroptosis.

First, we wanted to analyze whether MLKL upregulation was limited to specific cancer cell lines or to type I or type II IFN signaling. Next, we aimed to further understand the mechanisms underlying IFN-induced upregulation of MLKL by analyzing mRNA and protein level of MLKL and regulators of the IFN $\gamma$ pathway. We were especially interested whether MLKL is upregulated transcriptionally or due to caspase activation. In the end, we wanted to analyze the role of the IFN-induced transcription factors IRF1 and STAT1 in upregulation of MLKL by performing knockdown and knockout experiments.

In the second part, we focussed on the immune response to necroptotic cells in comparison to apoptotic cells. Whereas efferocytosis of apoptotic cells and polarization of macrophages in response to apoptotic cells is studied in detail, the effects of necroptotic cells on immune cells and especially macrophages are not well studied yet.

To exclude induction of other signaling pathways either in dying cells or immune cells, we used pure cell death systems of necroptosis and apoptosis. We aimed to analyze the genetic profile of macrophages after contact with dying cells in an unbiased approach using RNA sequencing. For this purpose, we established a co-culture system of macrophages and apoptotic or necroptotic cells. Finally, we confirmed the results of the RNA sequencing in vitro and in an in vivo model of self-resolving inflammation. Hereby, modulation of cell death during the Zymosan-A-induced peritonits by either injection of dying cells or cell death inhibitors was performed. 


\section{Material and Methods}

\subsection{Material}

\subsubsection{Cell lines}

Table 1. Human cell lines.

\begin{tabular}{lll}
\hline Cell line & Type & Source \\
EFM-192A & Breast tumor & DSMZ \\
HEK 293T & $\begin{array}{l}\text { Embryonic kidney cells } \\
\text { with SV40 T-antigen }\end{array}$ & ATCC \\
& Cervical cancer & DSMZ \\
HeLa & $\begin{array}{l}\text { Colorectal } \\
\text { adenocarcinoma }\end{array}$ & DSMZ \\
HT-29 & Adenocarcinoma & ATCC \\
MDA-MB-231 & $\begin{array}{l}\text { Biphenotypic B } \\
\text { myelomonocytic leukemia }\end{array}$ & DSMZ \\
MV4-11 & & \\
\hline
\end{tabular}

Table 2. Murine cell lines.

\begin{tabular}{lll}
\hline Cell line & Type & Source \\
IC-21 & Peritoneal macrophages & ATCC \\
NIH 3T3 & Embryonic fibroblasts & DSMZ \\
\hline
\end{tabular}

\subsubsection{Cell culture reagents}

Table 3. Cell culture reagents.

\begin{tabular}{ll}
\hline Reagent & Supplier \\
$\begin{array}{l}\text { Dulbecco's Modified Eagle Medium } \\
(\text { DMEM) }\end{array}$ & Life Technologies, Darmstadt, Germany \\
$\begin{array}{l}\text { Dulbecco's phosphate-buffered saline } \\
\text { (DPBS) }\end{array}$ & Life Technologies, Darmstadt, Germany \\
\hline
\end{tabular}


Fetal calf serum (FCS)

Penicillin/Streptomycin (10000 U/ml)

Puromycin

Roswell Park Memorial Institute Medium Life Technologies, Darmstadt, Germany (RPMI) 1640

RPMI 1640 [-] Phenol Red

Sodium Pyruvate

Trypan blue solution, $0.4 \%$

Trypsin/EDTA solution (0.05\%), phenol red
Life Technologies, Darmstadt, Germany

Life Technologies, Darmstadt, Germany

TaKaRa Bio/Clontech, Mountain View, CA, USA

Life Technologies, Darmstadt, Germany

Life Technologies, Darmstadt, Germany

Life Technologies, Darmstadt, Germany

Life Technologies, Darmstadt, Germany

\subsubsection{Drugs and inhibitors}

Table 4. List of drugs.

\begin{tabular}{ll}
\hline Reagent & Supplier \\
B/B Homodimerizer (dimerizer) & $\begin{array}{l}\text { TaKaRa Bio/Clontech, Mountain View, } \\
\text { CA, USA }\end{array}$ \\
Interferon $\alpha($ IFN $\alpha)$ & Sigma/Merck, Darmstadt, Germany \\
Interferon $\beta($ IFN $\beta)$ & Sigma/Merck, Darmstadt, Germany \\
Interferon $\gamma($ IFN $\gamma)$ & Merck/Biochrome, Darmstadt, Germany \\
BV6 & Genentech, San Francisco, CA, USA \\
\hline
\end{tabular}

Table 5. List of inhibitors.

\begin{tabular}{lll}
\hline Inhibitor & Target & Supplier \\
N-benzyloxycarbonyl-Val- & Caspases & Bachem, Heidelberg, \\
Ala-Asp (O-Me) & & Germany \\
fluoromethylketone & & \\
(zVAD.fmk) & & \\
GSK'872 (GSK) & RIPK3 & Merck, Darmstadt, \\
& & Germany \\
\hline
\end{tabular}




\begin{tabular}{lll}
\hline Necrostatin-1s (Nec-1s) & RIPK1 & $\begin{array}{l}\text { Calbiochem, Darmstadt, } \\
\text { Germany }\end{array}$ \\
Necrosulfonamid (NSA) & MLKL & $\begin{array}{l}\text { Calbiochem, Darmstadt, } \\
\text { Germany }\end{array}$ \\
Actinomycin-D & transcription & Sigma-Aldrich, Darmstadt, \\
& & Germany \\
\hline
\end{tabular}

\subsubsection{Fluorecent dyes}

Table 6. List of fluorescent dyes.

\begin{tabular}{ll}
\hline Reagent & Supplier \\
eFluor 670 & Invitrogen, Carlsbad, CA, USA \\
Hoechst 33342 & Thermo Fisher Scientific, Waltham, MA, \\
& USA \\
Propidium iodide (PI) & Sigma-Aldrich, Darmstadt, Germany \\
\hline
\end{tabular}

\subsubsection{Antibodies}

Table 7. Primary antibodies for Western Blotting.

\begin{tabular}{|c|c|c|c|}
\hline Antibody & Species & Dilution & Supplier \\
\hline Anti- $\beta$-Actin & Mouse & $1: 5000$ & Sigma-Aldrich, Darmstadt, Germany \\
\hline Anti-FLAG & Mouse & $1: 1000$ & Sigma-Aldrich, Darmstadt, Germany \\
\hline Anti-GAPDH & Mouse & $1: 5000$ & Hytest, Turku, Finland \\
\hline Anti-GFP & Rabbit & $1: 5000$ & $\begin{array}{l}\text { TaKaRa Bio/Clontech, Mountain } \\
\text { View, CA, USA }\end{array}$ \\
\hline $\begin{array}{l}\text { Anti-human } \\
\text { Caspase } 8\end{array}$ & Mouse & $1: 2000$ & Enzo, Lörrach, Germany \\
\hline Anti-IRF1 & Rabbit & $1: 1000$ & Abcam, Cambridge, UK \\
\hline Anti-MLKL & Rabbit & $1: 5000$ & Cell Signaling, Beverly, MA, USA \\
\hline $\begin{array}{l}\text { Anti-murine } \\
\text { RIPK3 }\end{array}$ & Rabbit & $1: 1000$ & Axxora, Lörrach, Germany \\
\hline Anti-pMLKL & Rabbit & $1: 1000$ & Cell Signaling, Beverly, MA, USA \\
\hline
\end{tabular}




\begin{tabular}{llll}
\hline Anti-pSTAT1 & Rabbit & $1: 1000$ & Cell Signaling, Beverly, MA, USA \\
Anti-STAT1 & Mouse & $1: 1000$ & Cell Signaling, Beverly, MA, USA \\
Anti-Vinculin & Mouse & $1: 2000$ & Merck, Darmstadt, Germany \\
\hline
\end{tabular}

Table 8 . Secondary antibodies for Western Blotting.

\begin{tabular}{|c|c|c|c|}
\hline Antibody & Species & Dilution & Supplier \\
\hline Anti-Mouse IRDye 680LT & Donkey & $1: 10000$ & $\begin{array}{l}\text { LI-COR Biosciences, } \\
\text { Lincoln, NE, USA }\end{array}$ \\
\hline Anti-Mouse IRDye $800 \mathrm{CW}$ & Donkey & $1: 10000$ & $\begin{array}{l}\text { LI-COR Biosciences, } \\
\text { Lincoln, NE, USA }\end{array}$ \\
\hline Anti-Rabbit IRDye 680LT & Donkey & $1: 10000$ & $\begin{array}{l}\text { LI-COR Biosciences, } \\
\text { Lincoln, NE, USA }\end{array}$ \\
\hline Anti-Rabbit IRDye $800 \mathrm{CW}$ & Donkey & $1: 10000$ & $\begin{array}{l}\text { LI-COR Biosciences, } \\
\text { Lincoln, NE, USA }\end{array}$ \\
\hline HRP-conjugated anti-mouse-IgG & Goat & $1: 10000$ & Abcam, Cambridge, UK \\
\hline HRP-conjugated anti-rabbit-IgG & Goat & $1: 10000$ & Abcam, Cambridge, UK \\
\hline
\end{tabular}

Table 9. Antibodies for flow cytometry.

\begin{tabular}{llll}
\hline Marker & Dye & $\begin{array}{l}\text { Amount per } \\
\text { sample }\end{array}$ & Supplier \\
Annexin-V & APC & $2 \mu 1$ & $\begin{array}{l}\text { Biolegend, San Diego, CA, } \\
\text { USA }\end{array}$ \\
Annexin-V & FITC & $0.2 \mu 1$ & $\begin{array}{l}\text { In-house production } \\
\text { part I }\end{array}$ \\
$\begin{array}{llll}\text { Annexin-V } \\
\text { part II }\end{array}$ & FITC & $2 \mu 1$ & $\begin{array}{l}\text { ImmunoTools, Friesoythe; Ger- } \\
\text { many }\end{array}$ \\
CD117 & FITC & $0.5 \mu 1$ & $\begin{array}{l}\text { BD Bioscience, Heidelberg, } \\
\text { Germany }\end{array}$ \\
CD11b & BV605 & $0.5 \mu 1$ & $\begin{array}{l}\text { BD Bioscience, Heidelberg, } \\
\text { Germany }\end{array}$ \\
& & & $\begin{array}{l}\text { BD Bioscience, Heidelberg, } \\
\text { Germany }\end{array}$ \\
CD11c & AlexaFluor & $1.5 \mu 1$ & \\
& 700 & &
\end{tabular}




\begin{tabular}{|c|c|c|c|}
\hline CD140a & $\mathrm{PE}$ & $2 \mu \mathrm{l}$ & $\begin{array}{l}\text { BD Bioscience, Heidelberg, } \\
\text { Germany }\end{array}$ \\
\hline CD16/32 & none & $2 \mu 1$ & $\begin{array}{l}\text { BD Bioscience, Heidelberg, } \\
\text { Germany }\end{array}$ \\
\hline CD19 & APC-H7 & $1 \mu \mathrm{l}$ & $\begin{array}{l}\text { Biolegend, San Diego, CA, } \\
\text { USA }\end{array}$ \\
\hline CD3 & PE-CF594 & $1.5 \mu \mathrm{l}$ & $\begin{array}{l}\text { BD Bioscience, Heidelberg, } \\
\text { Germany }\end{array}$ \\
\hline CD34 & FITC & $1 \mu 1$ & $\begin{array}{l}\text { BD Bioscience, Heidelberg, } \\
\text { Germany }\end{array}$ \\
\hline $\mathrm{CD} 4$ & BV711 & $1 \mu 1$ & $\begin{array}{l}\text { Biolegend, San Diego, CA, } \\
\text { USA }\end{array}$ \\
\hline CD45 & VioBlue & $2 \mu 1$ & $\begin{array}{l}\text { Miltenyi Biotech, Bergisch- } \\
\text { Gladbach, Germany }\end{array}$ \\
\hline CD8 & BV650 & $0.5 \mu 1$ & $\begin{array}{l}\text { Biolegend, San Diego, CA, } \\
\text { USA }\end{array}$ \\
\hline CD90.2 & $\mathrm{PE}$ & $1 \mu \mathrm{l}$ & $\begin{array}{l}\text { Miltenyi Biotech, Bergisch- } \\
\text { Gladbach, Germany }\end{array}$ \\
\hline $\mathrm{F} 4 / 80$ & PE-Cy7 & $1 \mu 1$ & $\begin{array}{l}\text { Biolegend, San Diego, CA, } \\
\text { USA }\end{array}$ \\
\hline GITR & FITC & $1 \mu 1$ & $\begin{array}{l}\text { Biolegend, San Diego, CA, } \\
\text { USA }\end{array}$ \\
\hline $\begin{array}{l}\text { HLA-DR } \\
(\mathrm{MHC} \text { II) }\end{array}$ & APC & $1 \mu \mathrm{l}$ & $\begin{array}{l}\text { Biolegend, San Diego, CA, } \\
\text { USA }\end{array}$ \\
\hline Ly-6C & PerCP-Сy5.5 & $1 \mu 1$ & $\begin{array}{l}\text { Biolegend, San Diego, CA, } \\
\text { USA }\end{array}$ \\
\hline Ly-6G & APC-Cy7 & $1.5 \mu \mathrm{l}$ & $\begin{array}{l}\text { Biolegend, San Diego, CA, } \\
\text { USA }\end{array}$ \\
\hline NK1.1 & $\begin{array}{l}\text { AlexaFluor } \\
700\end{array}$ & $1.5 \mu \mathrm{l}$ & $\begin{array}{l}\text { BD Bioscience, Heidelberg, } \\
\text { Germany }\end{array}$ \\
\hline
\end{tabular}




\subsubsection{Plasmids}

Table 10. List of plasmids.

\begin{tabular}{lll}
\hline Vector backbone & Transgene/Content & Source \\
pBabe & Empty & $\begin{array}{l}\text { Kindly provided by A. } \\
\text { Oberst [24] }\end{array}$ \\
pBabe & FV-hCasp8-2A-GFP & \\
pBabe & FLAG-mRIPK3-dC- & \\
& 2xFV-2A-GFP & \\
pLentiCRISPRv2 & lentiviral backbone & Addgene plasmid \# 52961 \\
psPAX2 & $\begin{array}{l}\text { lentiviral packaging } \\
\text { plasmid }\end{array}$ & Addgene plasmid \# 12260 \\
& VSV-G envelope & Addgene plasmid \# 12259 \\
pMD2.G & expressing plasmid & \\
\hline
\end{tabular}

\subsubsection{Primer}

Table 11. List of human RT-PCR primer.

\begin{tabular}{ll}
\hline Primer & Sequence \\
IRF-1-fwd & ACAGCACCAGTGATCTGTACAAC \\
IRF-1 rev & TTCCCTTCCTCATCCTCATCT \\
MLKL-fwd & CCCATGATCCCTCTGTGC \\
MLKL-rev & GACTCCTTGGTTTAGATTTTGATACAC \\
RIPK1-fwd & GTGTACAAGGGGCCCAACT \\
RIPK1-rev & CGGCTGTGTCTCAGTCTGTT \\
RIPK3-fwd & GCCTCCACAGCCAGTGAC \\
RIPK3-rev & TCGGTTGGCAACTCAACTT \\
\hline
\end{tabular}

Table 12. List of murine RT-PCR primer.

\section{Primer}

Alox-15-fwd

Alox-15-rev
Sequence

AGAGTGGCCACACCAAGATG

AAGTAGACCGCTTCAGCACC 


\begin{tabular}{ll}
\hline Arg-1-fwd & ACGGCAGTGGCTTTAACCTT \\
Arg-1-rev & AGGTAGTCAGTCCCTGGCTT \\
Aurka-fwd & AGACCACTGTTCCCTTCGGTCC \\
Aurka-rev & CTGGCCACTGCTAGCAGATCCT \\
Aurkb-fwd & GTCTGGCCTGAACACGTTGTCC \\
Aurkb-rev & GGACTGGCTGTTGAACCGGTTC \\
Ccnb1-fwd & CTGAGCCTGAGCCTGAACCTGA \\
Ccnb1-rev & CCATCGGGCTTGGAGAGGGATT \\
CD206-fwd & CCATCTCAGTTCAGACGGCA \\
CD206-rev & ACGGAAGCCCAGTCAGTTTT \\
Cdk1-fwd & CGAGGAAGAAGGAGTGCCCAGT \\
Cdk1-rev & AGCACATCCTGCAGGCTGACTA \\
IL-6-fwd & CCGGAGAGGAGACTTCACAG \\
IL-6-rev & TTCTGCAAGTGCATCATCGT \\
Mki67-fwd & AGGAATCGCGGGAGACACAGCT \\
Mki67-rev & CCATTCCACCGCGCCATCTCTC \\
TNF-fwd & GCTGAGCTCAAACCCTGGTA \\
TNF-rev & CGGACTCCGCAAAGTCTAAG \\
Top2a-fwd & \\
\hline Top2a-rev & \\
\hline
\end{tabular}

\subsubsection{Small-interfering RNAs (siRNAs)}

siRNAs were purchased from Invitrogen (Karlsruhe, Germany).

Table 13. List of si-RNAs.

\begin{tabular}{llll}
\hline siRNA & Sequence code & Target & Concentration \\
IRF1 \#1 & s7501 & IRF1 & $20-40 \mathrm{nM}$ \\
IRF1 \#2 & s7502 & IRF1 & $20-40 \mathrm{nM}$ \\
siCtrl & 4390844 & $\begin{array}{l}\text { Non-targeting } \\
\text { control }\end{array}$ & $20-40 \mathrm{nM}$ \\
& & & \\
\hline
\end{tabular}




\begin{tabular}{llll}
\hline STAT1 \#1 & s277 & STAT1 & $20-40 \mathrm{nM}$ \\
STAT1 \#2 & $\mathrm{s} 278$ & STAT1 & $20-40 \mathrm{nM}$ \\
\hline
\end{tabular}

3.1.9. Guide RNAs (gRNAs)

Table 14. List of gRNAs.

\begin{tabular}{lll}
\hline gRNA & Target & Sequence \\
IRF1 gRNA1 & IRF1 & TTAATTCCAACCAAATCCCG \\
IRF1 gRNA2 & IRF1 & TCTAGGCCGATACAAAGCAG \\
IRF1 gRNA3 & IRF1 & GAACTCCCTGCCAGATATCG \\
\hline
\end{tabular}

3.1.10. Reagents and kits

Table 15. List of reagents and kits.

\begin{tabular}{ll}
\hline Reagent/kit & Supplier \\
Agilent DNA High Sensitivity DNA & $\begin{array}{l}\text { Agilent Technologies, Waldbronn, } \\
\text { Chip }\end{array}$ \\
Germany \\
Brilliant Stain Buffer & BD Bioscience, Heidelberg, Germany \\
CellEvent Caspase 3/7 Green Detection & Thermo Fisher Scientific, Waltham, MA, \\
Reagent & USA \\
cOmplete Protease Inhibitor Cocktail & Roche Diagnostics, Mannheim, Germany \\
(PIC), EDTA free (25x) & \\
FACS Clean/Rinse Solution & BD Bioscience, Heidelberg, Germany \\
FACS Flow Sheath Fluid & BD Bioscience, Heidelberg, Germany \\
FACS Shutdown Solution & BD Bioscience, Heidelberg, Germany \\
FcR Blocking Reagent mouse & Miltenyi Biotech, Bergisch-Gladbach, \\
& Germany \\
Flow Cytometry Absolute Count & Bangs Laboratories, INC., Polysciences \\
Standard & Europe GMBH, Hirschberg an der \\
& Bergstrasse, Germany \\
FuGene HD Transfections Reagent & Promega, Mannheim, Germany \\
\hline
\end{tabular}


GeneJet Plasmid Miniprep Kit

High Output Kit v2

Lipofectamine RNAi MAX

Nuclease-free water

One Shot Top 10 chemically competent E.coli

OptiMEM transfection medium

PageRulerTM Plus Prestained Protein

Ladder

peqGOLD Total RNA kit

Pierce $^{\mathrm{TM}}$ BCA Protein Assay

Pierce $^{\mathrm{TM}}$ ECL Western Blotting Substrate

Pierce ${ }^{\mathrm{TM}}$ Universal Nuclease

PureLink $^{\mathrm{TM}}$ HiPure Plasmid Maxiprep

Kit

Qiagen RNeasy Micro Kit

QuantSeq 3' mRNA-Seq Library Prep Kit with the UMI Second Strand Synthesis

Module

Qubit dsDNA HS Assay Kit

Qubit HS RNA Assay Kit

RevertAid H Minus First Stand cDNA Synthesis Kit

RNA 6000 Pico chip

ROENTEROLL HC X-Ray Developer
Thermo Fisher Scientfic, Waltham, MA, USA

Illumina, San Diego, CA, USA

Invitrogen, Carlsbad, CA, USA

Thermo Fisher Scientfic, Waltham, MA, USA

Thermo Fisher Scientfic, Waltham, MA, USA

Thermo Fisher Scientfic, Waltham, MA, USA

Thermo Fisher Scientfic, Waltham, MA, USA

Peqlab, Erlangen, Germany

Thermo Fisher Scientfic, Waltham, MA, USA

Thermo Fisher Scientfic, Waltham, MA, USA

Thermo Fisher Scientfic, Waltham, MA, USA

Thermo Fisher Scientfic, Waltham, MA, USA

Qiagen, Hilden, Germany

Lexogen, Vienna, Austria

Thermo Fisher Scientfic, Waltham, MA, USA

Thermo Fisher Scientfic, Waltham, MA, USA

MBI Fermentas GmbH, St. Leon-Rot, Germany

Agilent Technologies, Waldbronn, Germany

Tetenal, Norderstadt, Germany 
S.O.C Medium

SUPERFIX MRP X-Ray Fixer

SYBR-green based quantitative real-time PCR

Zymosan-A
Life Technologies, Darmstadt, Germany

Tetenal, Norderstadt, Germany

Applied Biosystems, Darmstadt, Germany

Sigma-Aldrich, Darmstadt, Germany

\subsubsection{Chemicals}

Table 16. List of chemicals.

\section{Chemical}

2-propanol

Acetone

Acrylamide mix, $30 \%$ (Rotiphorese)

Agar

Albumin fraction V (BSA)

Ammonium chloride $\left(\mathrm{NH}_{4} \mathrm{Cl}\right)$

Ammonium persulfate (APS)

Antimycin A

Bromophenolblue

Calcium chloride $\left(\mathrm{CaCl}_{2}\right)$

Carbonyl cyanide 4-

(trifluoromethoxy)phenylhydrazone (FCCP)

Dimethyl sulfoxide (DMSO)

Disodium phosphate $\left(\mathrm{Na}_{2} \mathrm{HPO}_{4}\right)$

Dithiothreitol (DTT)

Ethanol

Ethylene diamine tetraacetic acid (EDTA)

Glycerol

Glycine

\section{Supplier}

Carl Roth, Karlsruhe, Germany

Carl Roth, Karlsruhe, Germany

Carl Roth, Karlsruhe, Germany

Carl Roth, Karlsruhe, Germany

Carl Roth, Karlsruhe, Germany

Carl Roth, Karlsruhe, Germany

Carl Roth, Karlsruhe, Germany

Sigma-Aldrich, Darmstadt, Germany

Carl Roth, Karlsruhe, Germany

Carl Roth, Karlsruhe, Germany

Cayman, Ann Arbor, MI, USA

Sigma-Aldrich, Darmstadt, Germany

Carl Roth, Karlsruhe, Germany

Millipore, Darmstadt, Germany

Carl Roth, Karlsruhe, Germany

Carl Roth, Karlsruhe, Germany

Carl Roth, Karlsruhe, Germany

Carl Roth, Karlsruhe, Germany 


\section{HEPES}

Hexadimethrine bromide (polybrene)

Hydrochloric acid ( $\mathrm{HCl})$

LB medium

Magnesium chloride $\left(\mathrm{MgCl}_{2}\right)$

Magnesium sulfate $\left(\mathrm{MgSO}_{4}\right)$

Methanol

Milk powder

Oligomycin

Phenylmethylsulfonyl fluoride (PMSF)

Potassium chloride $(\mathrm{KCl})$

Potassium dihydrogen phosphate

$\left(\mathrm{KH}_{2} \mathrm{PO}_{4}\right)$

Rotenone

Sodium bicarbonate $\left(\mathrm{NaHCO}_{3}\right)$

Sodium chloride $(\mathrm{NaCl})$

Sodium deoxycholate

Sodium dodecyl sulfate (SDS)

Sodium fluoride

Sodium hydroxide $(\mathrm{NaOH})$

Sodium orthovanadate

Tetramethylethylendiamine (TEMED)

Thiazolyl Blue Tetrazolium Bromide (MTT)

Tris Base

Tris $\mathrm{HCl}$

Triton X-100

Tween20

$\beta$-Glycerophosphate
Carl Roth, Karlsruhe, Germany

Carl Roth, Karlsruhe, Germany

Carl Roth, Karlsruhe, Germany

Carl Roth, Karlsruhe, Germany

Carl Roth, Karlsruhe, Germany

Carl Roth, Karlsruhe, Germany

Carl Roth, Karlsruhe, Germany

Carl Roth, Karlsruhe, Germany

Sigma-Aldrich, Darmstadt, Germany

Carl Roth, Karlsruhe, Germany

Carl Roth, Karlsruhe, Germany

Carl Roth, Karlsruhe, Germany

Sigma-Aldrich, Darmstadt, Germany

Carl Roth, Karlsruhe, Germany

Carl Roth, Karlsruhe, Germany

Thermo Fisher Scientific, Waltham, MA, USA

Carl Roth, Karlsruhe, Germany

Sigma-Aldrich, Darmstadt, Germany

Carl Roth, Karlsruhe, Germany

Sigma-Aldrich, Darmstadt, Germany

Carl Roth, Karlsruhe, Germany

Sigma-Aldrich, Darmstadt, Germany

Carl Roth, Karlsruhe, Germany

Carl Roth, Karlsruhe, Germany

Carl Roth, Karlsruhe, Germany

Carl Roth, Karlsruhe, Germany

Sigma-Aldrich, Darmstadt, Germany 


\subsubsection{Buffers}

Table 17. List of buffers and ingredients.

\begin{tabular}{|c|c|}
\hline Buffer & Ingredients \\
\hline \multirow[t]{5}{*}{ Annexin buffer } & 10 mM HEPES pH 7.4 \\
\hline & $150 \mathrm{mM} \mathrm{NaCl}$ \\
\hline & $5 \mathrm{mM} \mathrm{KCl}$ \\
\hline & $1 \mathrm{mM} \mathrm{MgCl}_{2}$ \\
\hline & $1.8 \mathrm{mM} \mathrm{CaCl}_{2}$ \\
\hline Blocking buffer & $5 \%$ milk powder in PBS-T \\
\hline \multirow[t]{4}{*}{ Blotting buffer } & $5.8 \mathrm{~g} / 1$ Tris Base \\
\hline & $2.9 \mathrm{~g} / \mathrm{l}$ glycine \\
\hline & $0.04 \% \mathrm{SDS}$ \\
\hline & $20 \%$ methanol \\
\hline \multirow[t]{3}{*}{ Erythrocyte lysis buffer ( $\mathrm{pH} 7.4$ ) } & $155 \mathrm{mM} \mathrm{NH}_{4} \mathrm{Cl}$ \\
\hline & $10 \mathrm{mM} \mathrm{NaHCO} 3$ \\
\hline & $0.1 \mathrm{mM}$ EDTA \\
\hline \multirow[t]{5}{*}{ Krebs Henseleit buffer } & $111 \mathrm{mM} \mathrm{NaCl}$ \\
\hline & $4.7 \mathrm{mM} \mathrm{KCl}$ \\
\hline & $1.25 \mathrm{mM} \mathrm{CaCl}_{2}$ \\
\hline & $2 \mathrm{mM} \mathrm{MgSO}_{4}$ \\
\hline & $1.2 \mathrm{mM} \mathrm{Na}_{2} \mathrm{HPO}_{4}$ \\
\hline PBS-Tween (PBS-T) & $1 x$ PBS, $0.1 \%$ Tween 20 \\
\hline \multirow{4}{*}{$\begin{array}{l}\text { Phosphate buffered saline (PBS, } 10 \mathrm{x}, \mathrm{pH} \\
\text { 7.4) }\end{array}$} & $80 \mathrm{~g} / 1 \mathrm{NaCl}$ \\
\hline & $2 \mathrm{~g} / 1 \mathrm{KCl}$ \\
\hline & $2 \mathrm{~g} / 1 \mathrm{KH}_{2} \mathrm{PO}_{4}$ \\
\hline & $14.4 \mathrm{~g} / 1 \mathrm{Na}_{2} \mathrm{HPO}_{4}$ \\
\hline \multirow[t]{6}{*}{ RIPA lysis buffer } & $50 \mathrm{mM}$ Tris $\mathrm{HCl} \mathrm{pH} 8.0$ \\
\hline & $1 \%$ Triton-X-100 \\
\hline & $0.5 \%$ Na-deoxycholate \\
\hline & $150 \mathrm{mM} \mathrm{NaCl}$ \\
\hline & $2 \mathrm{mM} \mathrm{MgCl}_{2}$ \\
\hline & $10 \% \mathrm{SDS}$ \\
\hline
\end{tabular}




\begin{tabular}{|c|c|}
\hline & Protease Inhibitor Cocktails (PIC) \\
\hline & $250 \mathrm{U} / \mathrm{mL}$ Pierce Universal Nuclease \\
\hline & $5 \mathrm{mM}$ Sodium fluoride \\
\hline & $1 \mathrm{mM} \beta$-glycerophosphate \\
\hline & $0.5 \mathrm{mM}$ PMSF \\
\hline & $1 \mathrm{mM}$ Sodium orthovanadate \\
\hline \multirow[t]{5}{*}{ SDS Loading buffer (6x) (pH 6.8) } & $350 \mathrm{mM}$ Tris Base \\
\hline & $3.8 \%$ glycerol \\
\hline & $10 \% \mathrm{SDS}$ \\
\hline & $0.093 \mathrm{~g} / \mathrm{ml}$ DTT \\
\hline & $0.12 \mathrm{mg} / \mathrm{ml}$ bromophenolblue \\
\hline \multirow[t]{3}{*}{ SDS Running buffer $(5 x)$} & $15.1 \mathrm{~g} / 1$ Tris Base \\
\hline & $94 \mathrm{~g} / 1$ glycine \\
\hline & $0.5 \% \mathrm{SDS}$ \\
\hline \multirow[t]{10}{*}{ Triton X-100 lysis buffer } & $30 \mathrm{mM}$ Tris $\mathrm{HCl}$ \\
\hline & $150 \mathrm{mM} \mathrm{NaCl}$ \\
\hline & $10 \%$ glycerol \\
\hline & $1 \%$ Triton $\mathrm{X}-100$ \\
\hline & $1 \times \mathrm{PIC}$ \\
\hline & $0.5 \mathrm{mM}$ PMSF \\
\hline & $2 \mathrm{mM}$ DTT \\
\hline & $1 \mathrm{mM}$ Sodium orthovanadate \\
\hline & $1 \mathrm{mM} \beta$-glycerophosphate \\
\hline & $5 \mathrm{mM}$ Sodium fluoride \\
\hline
\end{tabular}

\subsubsection{Consumables}

Table 18. List of consumables.

\begin{tabular}{ll}
\hline Consumable & Supplier \\
Aluminum foil & Carl Roth, Karlsruhe, Germany \\
Cell culture dishes $(6 \mathrm{~cm}, 10 \mathrm{~cm}, 14.5$ & $\begin{array}{l}\text { Greiner Bio-One, Frickenhausen, Ger- } \\
\mathrm{cm})\end{array}$ \\
\hline
\end{tabular}


Cell culture flasks $\left(25 \mathrm{~cm}^{2}, 75 \mathrm{~cm}^{2}, 175\right.$ $\mathrm{cm}^{2}$ )

Cell culture plates, transparent (96-well, 48-well, 24-well, 6-well)

Cell culture plates, white (96-well)

Cell scraper

Centrifuge tubes $(15 \mathrm{ml}, 50 \mathrm{ml})$

Combitips, all sizes

Cryogenic vials $(1.8 \mathrm{ml})$

Disposal bags

Filter tips, all sizes

Filters, sterile $(0.45 \mu \mathrm{M})$

Hybond ECL nitrocellulose membrane

Hyperfilm ECL

Microcentrifuge tubes $(0.5 \mathrm{ml}, 1.5 \mathrm{ml}, 2$ $\mathrm{ml})$

Nitrile gloves

Parafilm

Pasteur pipettes $(15 \mathrm{~cm}, 30 \mathrm{~cm})$

PCR tubes

Pipette tips, all sizes

Round-bottom tubes

Scalpels

Seahorse 96-well cell culture plate

Syringes ( $2 \mathrm{ml}, 5 \mathrm{ml}, 10 \mathrm{ml})$

Transwell system $(6.5 \mathrm{~mm}$ transwell with $5 \mu \mathrm{m}$ pore size)

Whatman paper
Greiner Bio-One, Frickenhausen, Germany

Greiner Bio-One, Frickenhausen, Germany

Greiner Bio-One, Frickenhausen, Germany

BD Bioscience, Heidelberg, Germany

Greiner Bio-One, Frickenhausen, Germany

Eppendorf, Hamburg, Germany

Starlab, Hamburg, Germany

Carl Roth, Karlsruhe, Germany

Starlab, Hamburg, Germany

Merck Millipore, Darmstadt, Germany

GE Healthcare, Buckinghamshire, UK

GE Healthcare, Buckinghamshire, UK

Starlab, Hamburg, Germany

Starlab, Hamburg, Germany

VWE, Deisenhofen, Germany

Carl Roth, Karlsruhe, Germany

Starlab, Hamburg, Germany

Starlab, Hamburg, Germany

BD Bioscience, Heidelberg, Germany

B. Braun, Melsungen, Germany

Agilent Technologies, Waldbronn, Germany

B. Braun, Melsungen, Germany

Corning, NY, USA

Thermo Fisher Scientific, Waltham, MA, USA 


\subsubsection{Equipment}

Table 19. List of equipment.

\begin{tabular}{|c|c|}
\hline Equipment & Supplier \\
\hline Autoclave V-15 & Systec, Pforzheim, Germany \\
\hline Centrifuge MIKRO $200 \mathrm{R}$ & Hettich, Baden-Baden, Germany \\
\hline Centrifuge ROTANTA $460 \mathrm{R}$ & Hettich, Baden-Baden, Germany \\
\hline Centrifuge ROTIXA 50 RS & Hettich, Baden-Baden, Germany \\
\hline $\mathrm{CO} 2$ incubator MCO-19AIC & Sanyo, Wehr, Germany \\
\hline Easypet@ 3 & Eppendorf, Hamburg, Germany \\
\hline Electronic analytical balance 770 & Kern, Balingen, Germany \\
\hline Electronic analytical balance EW & Kern, Balingen, Germany \\
\hline FACS Canto II & BD Bioscience, Heidelberg, Germany \\
\hline FACS LSRFortessa & BD Bioscience, Heidelberg, Germany \\
\hline Freezer $\left(-20^{\circ} \mathrm{C}\right)$ & $\begin{array}{l}\text { Ewald Innovationstechnik, Bad Nenn- } \\
\text { dorf, Germany }\end{array}$ \\
\hline Freezer $\left(-80^{\circ} \mathrm{C}\right)$ & Sanyo, Wehr, Germany \\
\hline Fridge $\left(4^{\circ} \mathrm{C}\right)$ & $\begin{array}{l}\text { Ewald Innovationstechnik, Bad Nenn- } \\
\text { dorf, Germany }\end{array}$ \\
\hline Glass plates for SDS-PAGE & Bio-Rad, München, Germany \\
\hline Heating block & Eppendorf, Hamburg, Germany \\
\hline Heating magnetic stirrer ARE & VELP scientifica, Usmate, Italy \\
\hline $\begin{array}{l}\text { HeraSafe class II biological safety cabi- } \\
\text { net }\end{array}$ & Kendro, Langenselbold, Germany \\
\hline ImageXpress micro XLS system & Molecular devices, Sunnyvale, CA, USA \\
\hline IncuCyte imaging system & Essen BioScience, Ann Arbor, MI, USA \\
\hline Infinite M100 microplate reader & Tecan, Crailsheim, Germany \\
\hline Innova 4230 bacteria shaker & $\begin{array}{l}\text { New Bruinswick Scientific, Edison, NJ, } \\
\text { USA }\end{array}$ \\
\hline Inolab@ $\mathrm{pH} 7310 \mathrm{pH}$ meter & WTW, Weilheim, Germany \\
\hline Magnetic rack & AMS Biotechnology, Abington, UK \\
\hline
\end{tabular}




\begin{tabular}{|c|c|}
\hline Mastercycler ${ }^{\circledR}$ pro & Eppendorf, Hamburg, Germany \\
\hline Microcentrifuge & Benning, Bocholt, Germany \\
\hline Microscope CKX41 & Olympus, Hamburg, Germany \\
\hline Multipipette $\mathbb{C}$ plus & Eppendorf, Hamburg, Germany \\
\hline Nalgene ${ }^{\circledR}$ Mr Frosty freezing container & Sigma-Aldrich, Darmstadt, Germany \\
\hline NanoDrop 1000 spectrophotometer & Peqlab, Erlangen, Germany \\
\hline Neubauer improved counting chamber & Carl Roth, Karlsruhe, Germany \\
\hline NextSeq 500 sequencer & Illumina, San Diego, CA, USA \\
\hline Odyssey Imaging System & $\begin{array}{l}\text { LI-COR Bioscience, Bad Homburg, } \\
\text { Germany }\end{array}$ \\
\hline $\begin{array}{l}\text { PerfectBlue }{ }^{\mathrm{TM}} \text { Dual Gel Twin L electro- } \\
\text { phoresis system }\end{array}$ & Peqlab, Erlangen, Germany \\
\hline $\begin{array}{l}\text { Pipette Research plus }(2.5 \mu 1,10 \mu 1,100 \\
\mu 1,200 \mu 1,1000 \mu \mathrm{l})\end{array}$ & Eppendorf, Hamburg, Germany \\
\hline PowerPac ${ }^{\mathrm{TM}}$ Universal power supply & Bio-Rad, München, Germany \\
\hline Quantstudio 7 flex real-time PCR system & $\begin{array}{l}\text { Applied Biosystems, Darmstadt, } \\
\text { Germany }\end{array}$ \\
\hline Rocking Shaker & MS-L, Wiesloch, Germany \\
\hline Roller mixer & Ratek, Victoria, Australia \\
\hline Seahorse 96 extracellular flux analyzer & $\begin{array}{l}\text { Agilent Technologies, Waldbronn, Ger- } \\
\text { many }\end{array}$ \\
\hline Thermomixer comfort & Eppendorf, Hamburg, Germany \\
\hline Trans-Blot $\subset$ SD Semi-Dry Transfer Cell & Bio-Rad, München, Germany \\
\hline Vacuum Pump HLC & Ditabis, Pforzheim, Germany \\
\hline Vortex mixer ZX classic & VELP scientifica, Usmate, Italy \\
\hline Water bath SWB20 & Medingen, Arnsdorf, Germany \\
\hline X-Ray cassette type G & Rego X-Ray, Augsburg, Germany \\
\hline
\end{tabular}




\subsubsection{Software}

Table 20. List of software.

\begin{tabular}{|c|c|}
\hline Software & Supplier \\
\hline Adobe Illustrator (25.2.1) & $\begin{array}{l}\text { Adobe Systems Software, Dublin, } \\
\text { Republic of Ireland }\end{array}$ \\
\hline Benchling & Benchling, San Francisco, CA, USA \\
\hline Bluebee Data Analysis Platform & Lexogen GmbH, Vienna, Austria \\
\hline EndNote $(\mathrm{X} 7.8)$ & Thomson Reuters, Toronto, Canada \\
\hline FACSDiva $^{\mathrm{TM}}(6.1 .3)$ & BD Bioscience, Heidelberg, Germany \\
\hline FlowJo (10.6.2.) & BD Bioscience, Heidelberg, Germany \\
\hline $\begin{array}{l}\text { Gene Ontology enrichment analysis and } \\
\text { visualization tool (GOrilla) }\end{array}$ & $\begin{array}{l}\text { Multi Knowledge Project, http://cbl- } \\
\text { gorilla.cs.technion.ac.il/ }[50,51]\end{array}$ \\
\hline GraphPad Prism ${ }^{\circledR}(7)$ & $\begin{array}{l}\text { GraphPad Software, San Diego, CA, } \\
\text { USA }\end{array}$ \\
\hline Gene set enrichment analysis (GSEA) & $\begin{array}{l}\text { project of UC San Diego and Broad } \\
\text { Institute, https://www.gsea- } \\
\text { msigdb.org/gsea/index.jsp }[52,53]\end{array}$ \\
\hline ImageJ $(1.52 \mathrm{e})$ & $\begin{array}{l}\text { National Institute of Health, Bethesda, } \\
\text { MD, USA }\end{array}$ \\
\hline IncuCyte $2020 \mathrm{~B}$ software & Essen BioScience, Ann Arbor, MI, USA \\
\hline Magellan Data Analysis (7.2) & Tecan, Crailsheim, Germany \\
\hline $\operatorname{MetaXpress}^{\mathcal{O}}(6 \cdot 5 \cdot 1.347)$ & Molecular Devices, Sunnyvale, CA, USA \\
\hline Microsoft Office 2013 & Microsoft, Redmond, WA, USA \\
\hline $\begin{array}{l}\text { Morpheus matrix visualization and } \\
\text { analysis software }\end{array}$ & $\begin{array}{l}\text { https://software.broadinstitute.org/mor- } \\
\text { pheus, } 20 \text { August } 2020\end{array}$ \\
\hline NanoDrop Software (3.8.1) & Peqlab, Erlangen, Germany \\
\hline Tecan i-control $^{\mathrm{TM}}(1.10)$ & Tecan, Crailsheim, Germany \\
\hline
\end{tabular}




\subsection{Methods}

\subsubsection{Cell culture}

Cell culture work was performed using a Laminar flow workbench providing sterile conditions. Cells were cultivated in a humidified atmosphere at $37{ }^{\circ} \mathrm{C}$ with $5 \% \mathrm{CO}_{2}$. EFM192A, MV4-11 cells, peritoneal macrophages and IC-21 cells were cultured in RPMI Medium 1640 and HeLa, HEK 293T, MDA-MB-231 and NIH 3T3 cells were cultured in DMEM Medium, each supplemented with 10-20\% fetal calf serum, $1 \%$ penicillin/streptomycin and $1 \%$ sodium pyruvate. Cell lines were authenticated by STR profiles and negatively tested for mycoplasma contamination.

For passaging, adherent cells (except for IC-21 cells) were washed with PBS and trypsin was added for 5-10 minutes to detach cells. Medium was applied to stop trypsinization and cells were transferred to a new cell culture flask (splitting ratio of 1:3-1:10, once or twice per week, depending on the cell line). IC-21 cells were washed twice with PBS, new medium was added and cells were incubated 10 minutes at RT. Cells were scraped carefully with a flexible cell scraper and splitted in a ratio of 1:2 once a week. Three times a week medium was exchanged to prevent acidification of IC-21 cells. The suspension cell line MV4-11 was passaged 1:3 twice per week by transferring cell suspension to a new cell culture flask with fresh medium.

\subsubsection{Freezing and thawing of cells}

For storage, one confluent cell culture flask was washed with PBS. Cells were trysinized, centrifuged (1800 rpm, 5 min at RT) and solved in $1 \mathrm{ml}$ medium containing 10\% DMSO. Cells were frozen in cryogenic vials using $\mathrm{Mr}$ Frosty freezing container at $-80^{\circ} \mathrm{C}$. Cells were stored for long term in a liquid nitrogen tank. Cells were thawed at $37^{\circ} \mathrm{C}$ in a waterbath and the cell suspension was added dropwise to $7 \mathrm{ml}$ of pre-warmed medium. Cells were centrifuged for $5 \mathrm{~min}$ at $1800 \mathrm{rpm}$ and RT, resuspended in medium and cultured as described above.

\subsubsection{Cell counting and seeding}

After trypsinization, $20 \mu \mathrm{l}$ of the cell suspension was mixed with $60 \mu 1$ of $0.4 \%(\mathrm{v} / \mathrm{v})$ trypan blue and counted using a Neubauer counting chamber. Cells were seeded in the following concentrations or densities for experiments: 
Table 21. Concentrations or densities used to seed cell lines.

\section{Cells/cell line}

EFM-192A

HEK 293T

HeLa

HT-29

IC-21

MDA-MB-231

MV4-11

NIH 3 T3

Peritoneal macrophages

\section{Concentration or density}

$0.3 \times 10^{5}$ cells $/ \mathrm{cm}^{2}$

$0.2 \times 10^{5}$ cells $/ \mathrm{cm}^{2}$

$0.2 \times 10^{5}$ cells $/ \mathrm{cm}^{2}$

$0.2 \times 10^{5}$ cells $/ \mathrm{cm}^{2}$

$0.5 \times 10^{5}$ cells $/ \mathrm{cm}^{2}$

$0.25 \times 10^{5}$ cells $/ \mathrm{cm}^{2}$

$1 \times 10^{5}$ cells $/ \mathrm{ml}$

$0.2 \times 10^{5}$ cells $/ \mathrm{cm}^{2}$

$0.5 \times 10^{5}$ cells $/ \mathrm{cm}^{2}$

\subsubsection{Treatment of cells in vitro}

Adherent cells were seeded as described above one day prior to the experiment. Treatment was administered by adding small amounts of substances to the medium or by replacing medium with fresh medium containg drugs. Suspension cells were treated on the day of beginning of the experiment by addition of substances to the medium. For co-culture of NIH 3T3 cells and peritoneal macrophages, macrophages were seeded in 24-well plates and NIH 3T3 cells in the inserts of transwell plates.

\subsubsection{Isolation of peritoneal macrophages}

Peritoneal macrophages were isolated from wild type C57-BL/6 mice by peritoneal lavage. Mice were killed via cervical dislocation. The skin of the abomen was cut open and $5 \mathrm{ml}$ of ice-cold PBS were injected slowly with a $27 \mathrm{G}$ needle into the peritoneum. To distribute the fluid, mice were rolled 10 times from side to side. PBS was extraced from the peritoneum with a $20 \mathrm{G}$ needle. Lavage was transferred to a $15 \mathrm{ml}$ falcon on ice followed by centrifugation for $10 \mathrm{~min}$ at $500 \mathrm{~g}$ and $4{ }^{\circ} \mathrm{C}$. During in vivo experiments, supernatant was aliquoted and frozen at $-80{ }^{\circ} \mathrm{C}$ for cytokine and chemokine analysis. The pellet was resuspended in $3 \mathrm{ml}$ erythrocyte lysis buffer. Lysis of erythrocytes was

performed for $5 \mathrm{~min}$ on ice. Reaction was stopped by filling the falcon up with ice-cold PBS and followed by centrifugation $\left(500 \mathrm{~g}, 5 \mathrm{~min}, 4^{\circ} \mathrm{C}\right)$. Cells were seeded at indicated 
densities for 1 to 6 hours in RPMI medium. To select macrophages, non-adherent cells were washed away with PBS (2-3 times) and RPMI medium was added.

\subsubsection{Transient transfections using siRNA}

Cells were transfected using reverse transfection protocol with 20 to $40 \mathrm{nM}$ Silencer Select siRNA, i.e. non-silencing siRNA or targeting siRNA (listed in Table 13) using Lipofectamine RNAi MAX and OptiMEM.

Lipofectamine and siRNAs were diluted in appropriate volumes of OptiMEM. Lipofectamine mix was added to siRNA mix and incubated 5 min at RT. The transfection mix was distributed to each well and cells were added. Cells were seeded twice as dence as described above for reverse transfection. Knockdown of proteins was monitored 48 or 72 hours after transfection via Western Blotting.

3.2.5. Generation of genetically modified cell lines

3.2.5.1. Generation of CRISPR/Cas9-mediated IRF-1 knockout MDA-MB-231 cells

MDA-MB-231 IRF1 knockout cells were generated using CRISPR/Cas9 system. Three IRF1 guide RNAs were designed with 5' and 3' BsmB1 restriction site overhangs (listed in Table 14), annealed and ligated into pLentiCRISPRv2. Lentiviral particles were generated by co-transfecting $1.1 \mu \mathrm{g}$ of each pLentiCRISPRv2 IRF1 gRNA, $2.7 \mu \mathrm{g}$ psPAX2 and $1 \mu \mathrm{g}$ pMD2.G in HEK293T cells using FuGENE transfection reagent and OptiMEM.

24 and 48 hours after transfection, supernatant was harvested and filtrated using a $0.45 \mu \mathrm{m}$ filter. $500 \mu \mathrm{l}$ of lentivial supernatant were added to a 6 well of MDA-MB-231 cells and incubated for 72 hours. Selection with $12 \mu \mathrm{g} / \mathrm{ml}$ puromycin was conducted for one week (medium exchange every 48 hours). IRF1 knockout efficiency was confirmed using Western blot analysis.

\subsubsection{Generation of mRIPK3-FV/FV-hCasp-8 expressing NIH 3 T3 cells}

Murine RIPK3 (mRIPK3-FV) or human caspase 8 (FV-hCasp8) expressing NIH 3T3 cells were generated via retroviral transfection using plasmids listed in Table 10. To 
increase the amount of plasmids, chemically competent TOP10 bacteria were transformed, expanded and plasmids were isolated. For transformation, bacteria were thawed on ice for 5 to $10 \mathrm{~min}$. $1 \mu$ of plasmid was added directly to bacteria and mixed gently. Vials were incubated on ice for $30 \mathrm{~min}$ and heatshock was performed for $30 \mathrm{sec}$ at $42^{\circ} \mathrm{C}$. Vials were placed on ice, $125 \mu$ pre-warmed S.O.C. medium was added and samples were shaked at $37^{\circ} \mathrm{C}$ for one hour at $225 \mathrm{rpm} .50 \mu \mathrm{l}$ of transformation were spread on a LB agar plate and plates were incubated at $37^{\circ} \mathrm{C}$ overnight. One clone of every plate/construct was picked and $300 \mathrm{ml}$ LB medium inoculated. Bacteria were cultivated overnight at $37^{\circ} \mathrm{C}$ and shaking followed by plasmid isolation using using Maxiprep according to the manufacturer's instructions.

HEK293T cells were transfected with $2 \mu$ g plasmid DNA per 6-well. For this purpose, $100 \mu \mathrm{l}$ OptiMEM were mixed with $6 \mu \mathrm{l} \mathrm{FuGENE}$ and incubated for $5 \mathrm{~min}$ at RT. This mixture was added to plasmid DNA and incubated for $15 \mathrm{~min}$ at RT. Transfection mixture was added dropwise to HEK293T cells. 24 hours after transfection, medium was exchanged. 48 and 72 hours after transfection, supernatant was collected, pooled and filtered through a $0.45 \mu \mathrm{m}$ filter. NIH $3 \mathrm{~T} 3$ cells were transduced with lentiviral supernatant by adding supernatant and $10 \mu \mathrm{g} / \mathrm{ml}$ polybrene for 72 hours. Selection was performed using $1 \mu \mathrm{g} / \mathrm{ml}$ puromycin for at least one week.

To generate single clones, cells were diluted and seeded in 96-well plates (1.2 cells per well). Cell colonies derived from a single cell were selected and expanded. From each construct 12 single clone derived cell lines were analyzed and two of each construct were choosen and used for later experiments.

\subsubsection{In vivo experiments}

\subsubsection{Animals}

Male and female C57-BL/6 mice (8 to 12 weeks old) were obtained from Charles River (Wilmington, Massachusetts, USA) and kept at ZFE (Zentrale Forschungseinrichtung, Johann-Wolfgang Goethe University, Frankfurt) under specific-pathogen-free (SPF) conditions on a constant $12 \mathrm{~h} \mathrm{light/dark}$ cycle with temperature at $20 \pm 1^{\circ} \mathrm{C}$. Food and water were given ad libitum. In vivo experiments were approved by the Regierungspräsidium Darmstadt with the approval number FU/1244 (22. July 2019). To 
reduce stress after transportation, mice were kept one week at ZFE before killing or starting the experiments.

\subsubsection{Zymosan-A-induced peritonitis}

Female or male C57-BL/6 mice, which were older than 2 month, younger than 3 month and weight more than $18 \mathrm{~g}$, were injected i.p. with $10 \mathrm{mg} / \mathrm{kg}$ of Zymosan-A from Saccharomyces cerevisiae to induce a self-solving peritonitis. Zymosan-A was solved in steril cold PBS and injected in $200 \mu \mathrm{l} /$ per mouse. Weight and health conditions of mice were controlled daily.

\subsubsection{Injection of dying cells and cell death inhibitors}

To study the influence of cell death on the resolution of inflammation, dying cells or cell death inhibitors were injected just before the peak of inflammation. NIH 3 T3 mRIPK3FV or FV-hCasp 8 cells were treated for 4 or 8 hours with $10 \mathrm{nM}$ dimerizer. 8 hours after injection of Zymosan-A, dying cells were injected i.p.. Therefore, cells were washed with PBS, trypsinzed, centriuged for $5 \mathrm{~min}$ at $1800 \mathrm{rpm}$ and resuspended in cold PBS. $2 \times 10^{6}$ cells, solved in $200 \mu \mathrm{l}$ PBS were injected per mouse. $200 \mu \mathrm{PBS}+5 \%$ DMSO was used as vehicle control. $1 \mathrm{mg} / \mathrm{kg}$ GSK' 872 and/or $10 \mathrm{mg} / \mathrm{kg}$ zVAD.fmk were solved in $200 \mu \mathrm{PBS}+5 \%$ DMSO and injected i.p. 8 hours after Zymosan-A injection. At day 1, 3 or 6 after Zyosan-A injection mice were killed via cervixal dislocation and cells and cytokines were extracted via peritoneal lavage as described above with $3 \mathrm{ml}$ ice-cold PBS. One tenth of the peritoneal cells was used for analysis of the immune cell profil, remaining cells were seeded to analyze the mRNA of macrophages.

\subsubsection{Analysis of immune cells in lavage}

To study the immune cell profile in the lavage, peritoneal cells were stained with different antibodies (listed in Table 9) and analyzed using flow cytometry (BD, LSRFortessa).

Cells, isolated from the peritoneum of mice as described in section 3.2.3., were centrifuged $\left(500 \mathrm{~g}, 5 \mathrm{~min}, 4^{\circ} \mathrm{C}\right)$, re-suspended in $50 \mu \mathrm{PBS}+0.5 \% \mathrm{BSA}+2 \mu \mathrm{FcR}$ blocking reagent per sample, vortexed and incubated for $10 \mathrm{~min}$ at $4{ }^{\circ} \mathrm{C}$. Antibodies were diluted in Brilliant Violet staining buffer (see Table 9). $50 \mu 1$ per sample were added to the cells and incubated for $25 \mathrm{~min}$ at $4{ }^{\circ} \mathrm{C}$ in the dark. Cells were washed using $600 \mu \mathrm{l}$ PBS $+0.5 \%$ BSA, centrifuged $\left(500 \mathrm{~g}, 5 \mathrm{~min}, 4{ }^{\circ} \mathrm{C}\right)$ and resuspended in $350 \mu \mathrm{FACS}$ Flow in FACS tubes. $20 \mu \mathrm{l}$ of count standard beads were added per sample and cells were 
measured using flow cytometry. Data were analyzed using FlowJo version 10.6.2. The gating strategy is depicted in Figure 7:
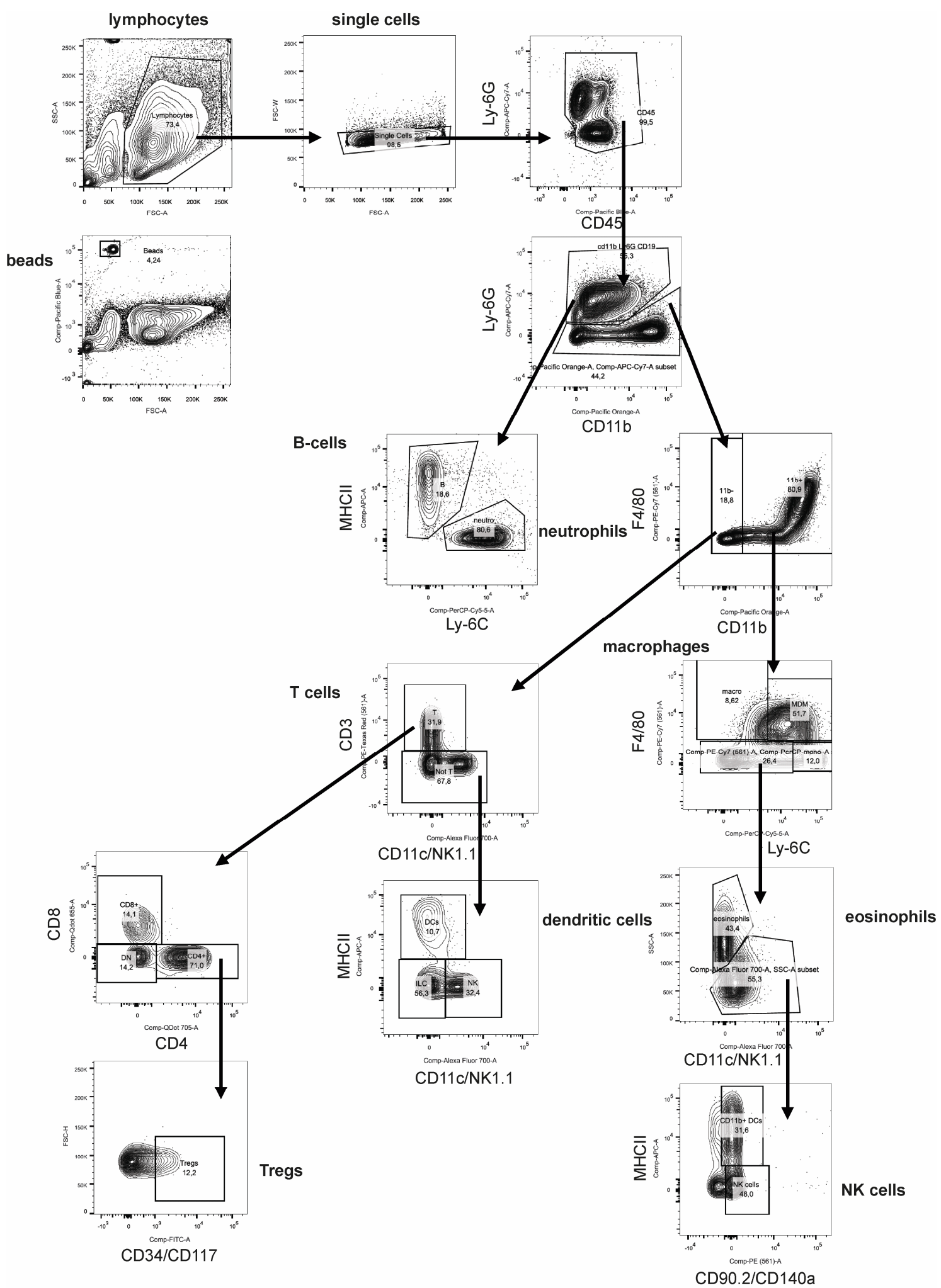

Figure 7. Gating strategy of immune cells isolated from mice. 


\subsubsection{Analysis of dead cells in lavage}

To analyze the percentage of dead cells in the lavage, peritoneal cells were stained with CD11b, CD45, MHC II, Ly-6G, PI and Annexin-V (ImmunoTools) antibodies and measured by flow cytometry (BD, LSRFortessa). Staining was performed as described in section 3.2.6.5. with the exception of using Annexin Buffer instead of Brilliant Violet staining buffer and washing with Annexin Buffer. Data were analyzed using FlowJo version 10.6.2.

\subsubsection{Cell death and cell viability assays}

\subsubsection{Determination of cell death using PI/Hoechst-staining}

Cell death was assessed by propidium iodide (PI)/Hoechst 33342 staining to determine plasma membrane permeability. Cells were seeded and treated in a 96 well plate as described above. After indicated time points $1 \mu \mathrm{g} / \mathrm{ml}$ PI and $10 \mu \mathrm{g} / \mathrm{ml}$ Hoechst 33342 were added and incubated for 5 to $10 \mathrm{~min}$. Fluorescence of PI (TRITC channel) and Hoechst (DAPI channel) was analyzed using ImageXpress Micro XLS system and MetaXpress software.

\subsubsection{Determination of cell death using Annexin V/PI co-staining}

Cell death were studied using Annexin V/PI co-staining and flow cytometry. Cells were seeded in 12- or 24-well plates as described above. After the indicated time points, cells were trypsinized and washed with PBS followed by centrifugation (1800 rpm, $5 \mathrm{~min}$, $4{ }^{\circ} \mathrm{C}$ ). Cells were resuspended in Annexin buffer containing $2 \mu$ Annexin-APC or $0.2 \mu \mathrm{l}$ Annexin-FITC (Inhouse production) and $1 \mu \mathrm{g} / \mathrm{ml} \mathrm{PI}$ and incubated for $10 \mathrm{~min}$ at RT. Cells were analyzed using FACS Canto II flow cytometer. Data were analyzed using FlowJo version 10.6.2.

\subsubsection{Determination of cell viability using MTT assay}

To study cell viability, a MTT assay was performed. Cells were seeded and treated in a 96-well plate as described above. At indicated timepoints, medium was removed using a vacuum pump. MTT (3-(4,5-dimethylthiazol-2-yl)-2,5-diphenyltetrazolium bromide) was mixed in a 1:10 dilution with RPMI without phenolred indicator and 100 $\mu$ MTT solution were added to each well. Plates were incubated for $3 \mathrm{~h}$ at $37^{\circ} \mathrm{C}$. MTT solution 
was removed and precipitated formazan was solved in $100 \mu 1$ isopropanol per well. Further solving was acessed by incubation for $30 \mathrm{~min}$ on a shaking table. Optical density was measured at $550 \mathrm{~nm}$ using a microplate reader and was normalized to control.

3.2.7.4. Determination of oxygen consumption rate and extracellular acidification rate

The cellular oxygen consumption rate (OCR) and extracellular acidification rate (ECAR) were analyzed using a Seahorse 96 extracellular flux analyzer. Peritoneal macrophages were co-cultured with NIH 3T3 cells for 48 hours and plated on a Seahorse 96-well cell culture plate at 100.000 cells/well at the day of measurement. $30 \mathrm{~min}$ prior to the measurement, macrophages were equilibrated in Krebs Henseleit buffer supplemented with $20 \mathrm{mM}$ L-glucose and $4 \mathrm{mM}$ L-glutamine. During measurement, cells were treated with $2.5 \mu \mathrm{M}$ oligomycin to block ATP-coupled respiration, $0.4 \mu \mathrm{M}$ Carbonyl cyanide 4(trifluoromethoxy)phenylhydrazone (FCCP) to uncouple the respiratory chain, and $1 \mu \mathrm{g} / \mathrm{ml}$ antimycin A together with $1 \mu \mathrm{M}$ rotenone to block mitochondrial respiration.

\subsubsection{Measurement of confluency using Incucyte}

To analyze proliferation of peritoneal macrophages after co-culture with NIH 3T3 FVhCasp 8 cells, confluency was measured every $4 \mathrm{~h}$ for $48 \mathrm{~h}$ using 10x objective in IncuCyte imaging system. Confluence was calculated using IncuCyte 2020B software.

\subsubsection{7. eFuor 670 proliferation assay}

Proliferation of IC-21 cells after co-culture with NIH 3T3 FV-hCasp8 cells was measured using eFluor 670 assay. IC-21 cells were stained with $5 \mu \mathrm{M}$ eFluor 670 according to the manufacturer's instructions before seeding and co-cultured with NIH 3T3 FV-hCasp8 cells as described above. After indicated time points, fluorescence intensity of IC-21 cells was measured using flow cytometry (FACS Canto II). Data were analyzed using FlowJo.

\subsubsection{Actinomycin D chase experiments}

To inhibit transcription, EFM-192A cells were pre-treated with $100 \mathrm{nM}$ of Actinomycin D. After 2 hours medium was exchanged and cells were treated with IFN $\gamma$ and Actinomycin D together for indicated time points. 


\subsubsection{RNA analysis}

\subsubsection{RT-PCR}

RNA was isolated using peqGOLD Total RNA kit according to the manufacturer's instructions. RNA concentration was determined using NanoDrop 1000 spectrophotometer. 0.5 to $1 \mu \mathrm{g}$ of total RNA were used for cDNA synthesis using the RevertAid H Minus First Stand cDNA Synthesis Kit according to the manufacturer's protocol with the use of random primers. $1 \mu \mathrm{l}$ of complementary DNA (cDNA) was used for quantification of gene expression levels. SYBR-green based quantitative real-time polymerase chain reaction (RT-PCR) was performed in duplicates using the 7900GR fast real-time PCR system. Data were normalized to house keeping gene mRNA expression. Relative expression levels of the target transcript were calculated compared to the reference transcript by using the $\Delta \Delta \mathrm{CT}$ method [54].

\subsubsection{RNA Sequencing}

All kits were used according to the manufacturer's instructions unless indicated otherwise. mRNA of macrophages was isolated using Qiagen RNeasy Micro Kit. RNA concentration was determined using Qubit HS RNA Assay Kit. Quality of isolated RNA was analyzed with RNA 6000 Pico chip. Library for RNA sequencing was prepared using QuantSeq 3' mRNA-Seq Library Prep Kit with the UMI Second Strand Synthesis Module for QuantSeq. Quality and concentration of DNA was checked using Agilent DNA High Sensitivity DNA Chip and Qubit dsDNA HS Assay Kit, respectively. Average size of each library was determined and the Library Quantifivation Calculation File from Lexogen used to calculate volumina. High Output Kit v2 was used to perform RNA sequencing on a NextSeq 500 sequencer. Data were analyzed using Bluebee Data Analysis Platform and mapped to mus musculus genome GRCm38 using Lexogen Quantseq 2.3.1. FWD UMI pipeline. Lexogen QuantSeq DE 1.3.1 pipeline was used to analyze differential gene expression. Gene ontology analysis (GOrilla [50, 51], base mean $>30$, p-value $<0.01, \operatorname{logfold}>2$ ) and gene set enrichment analysis (GSEA $[52,53]$ ) were used to identify differentially regulated pathways. Heatmaps were generated using Morpheus matrix visualization and analysis software. 


\subsubsection{Western Blot analysis}

To anaylze protein expression via Western Blotting, cells were seeded and treated as described above. Supernatant was collected, cells were scraped in PBS and suspension was transferred to falcons. After centrifugation for $5 \mathrm{~min}$ at $1800 \mathrm{rpm}$ and $4^{\circ} \mathrm{C}$, the cell pellet was washed once with PBS, centrifuged again and resolved in Triton X-100 lysis buffer or RIPA buffer. Cells were lysed for 25 to $35 \mathrm{~min}$ on ice and cell debris was removed by centrifugation $\left(20 \mathrm{~min}, 14000 \mathrm{rpm}, 4{ }^{\circ} \mathrm{C}\right.$ ). Protein concentration was determined using BCA kit using manufacturer's instructions. 50 to $100 \mu \mathrm{g}$ protein were filled up with $\mathrm{H}_{2} \mathrm{O}$ to an equal volume, loading dye was added and mixture was denatured for $5 \mathrm{~min}$ at $96{ }^{\circ} \mathrm{C}$. Samples and protein ladder were loaded onto homemade 10 to $12 \%$ SDS gels (5 \% stacking gel: $5 \%$ acrylamide, $125 \mathrm{mM}$ TrisHCl (pH 6.8), 0.1\% SDS, $0.1 \%$ APS, $0.1 \%$ TEMED; resolving gel: $10 \%$ or $12 \%$ acrylamide, $250 \mathrm{mM}$ TrisHCl (pH 8.8), $0.1 \%$ SDS, $0.1 \%$ APS, $0.04 \%$ TEMED).

To concentrate samples at the stacking gel, gels run slowly at $80 \mathrm{~V}$ for $15 \mathrm{~min}$ in running buffer. Separation was performed at $130 \mathrm{~V}$. A semi-dry blotting system was used to transfer proteins on nitrocellulose membranes in blotting buffer. Membranes were blocked with blocking buffer for 1 hour at RT.

Membranes were incubated with first antibodies in $2 \%$ BSA in PBS-T (except for antiIRF1 was incubated in $5 \%$ milk in PBS-T) over night at $4{ }^{\circ} \mathrm{C}$. Secondary antibodies conjugated to horseradish peroxidase or labeled with infrared dye were incubated in $5 \%$ milk in PBS-T or $2 \%$ BSA in PBS-T at RT for 1 hour and detected using enhanced chemiluminescence or infrared imaging. Representative blots of at least two independent experiments are shown.

\subsubsection{Cytokine and chemokine analysis}

\subsubsection{Cytometric bead array}

Concentrations of the cytokines IL-6, IL-10, TNF- $\alpha$ and IL-1 $\beta$ were analyzed with Cytometric bead array (CBA) and flow cytometry (BD, LSRFortessa) according to the manufacturer's instructions. $25 \mu \mathrm{l}$ of lavage or cell culture supernatant were centrifuged and used for CBA. 


\subsubsection{Legendplex assay}

Concentrations of 13 chemokines (CCL11, CCL17, CCL20, CXCL1, RANTES, CCL4, CCL22, CXCL5, CXCL9, CXCL10 and CXCL13) were analyzed using Legendplex assay and flow cytometry (BD, FACSCanto II) according to the manufacturer's instructions. $25 \mu \mathrm{l}$ of lavage or cell culture supernatant were centrifuged and used for Legendplex assay.

\subsubsection{Caspase $3 / 7$ activation assay}

Activation of caspase 3/7 was analyzed using CellEvent Caspase 3/7 Green Detection Reagent and Hoechst 33342 co-staining. Cells were seeded as decribed above and $2 \mu \mathrm{M}$ of caspase 3/7 detection reagent was added simultaneously. Cells were co-stained with $1 \mu \mathrm{g} / \mathrm{ml}$ Hoechst 33342 for 5 to $10 \mathrm{~min}$. Fluorescence was measured using ImageXpress Micro XLS system.

\subsubsection{Statistical analysis}

Statistical significance was assessed by Student's t-test (Two-tailed distribution, twosample, equal variance) using Microsoft Excel (Microsoft Deutschland GmbH, Unterschleißheim, Germany) or Annova using GraphPad Prism; ${ }^{*} \mathrm{p}<0.05 ; * * \mathrm{p}<0.01$; $* * * \mathrm{p}<0.001$. 


\section{Results}

\subsection{Part I: IFNs induce MLKL expression in cancer cells}

4.1.1. IFN $\gamma$ together with pan-caspase inhibitor $\mathrm{zVAD}$.fmk induced necroptosis and reduced cell viability of cancer cells

It was shown previously, that IFNs are able to induce necroptosis by activating the JAKSTAT pathway [8]. To confirm this finding, we treated EFM-192A and HeLa with different concentrations of IFN $\gamma$ and pan-caspase inhibitor zVAD.fmk (Figure 8). Cell death was induced in EFM-192A starting at $1 \mathrm{ng} / \mathrm{ml}$ IFN $\gamma$, but reached only 10 to $20 \%$ of all cells (Figure $8 \mathrm{~A}$ ). IFN $\gamma$ did not induce cell death in HeLa cells (Figure 8 B). This could be explained by the fact that HeLa cells do not express the necroptosis protein RIPK3. For later studies we used $1.5 \mathrm{ng} / \mathrm{ml} \mathrm{IFN} \gamma$ for EFM-192A and $10 \mathrm{ng} / \mathrm{ml} \mathrm{IFN} \gamma$ for HeLa cells.
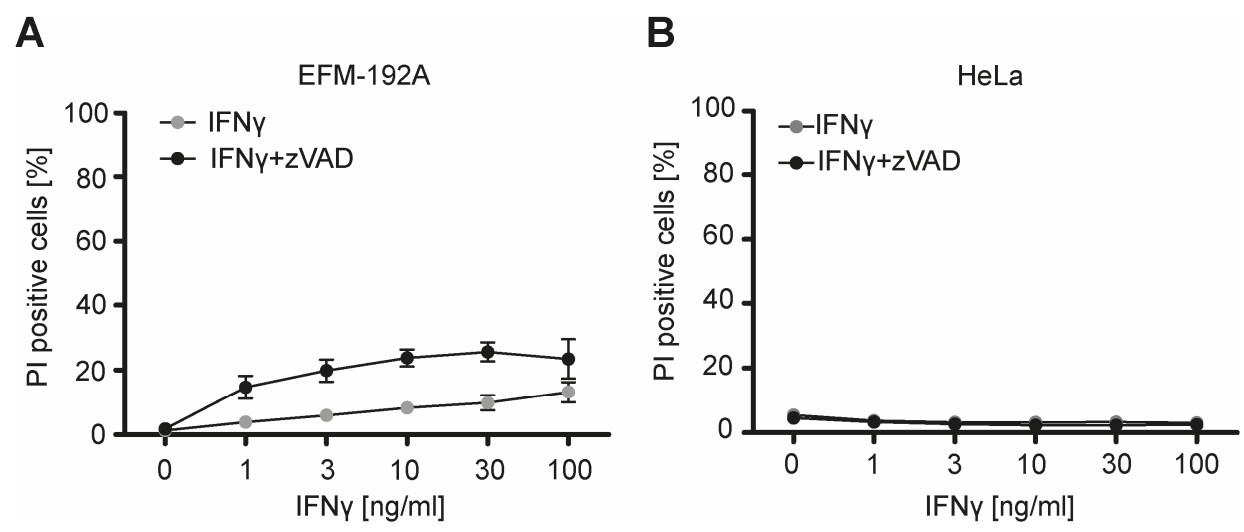

Figure 8. Treatment with IFN $\gamma$ and zVAD.fmk induced cell death in EFM-192A cells but not in HeLa cells. EFM-192A (A) and HeLa (B) cells were treated with different concentrations of IFN $\gamma$ and $20 \mu \mathrm{m}$ zVAD.fmk for 72 hours. Cell death was observed by counting PI positive cells. Data are shown as mean \pm standard error of the mean (SEM). $n=3-4$.

To test whether higher levels of cell death could be induced at other time points, EFM192A and HeLa cells were treated with IFN $\gamma$ and zVAD.fmk for different periods of time (Figure 9). Only the combination treatment of IFN $\gamma$ and zVAD.fmk could induce cell death in EFM-192A cells after 72 hours (Figure 9 A). This treatment did not induce cell death in HeLa cells (Figure 9 B). 

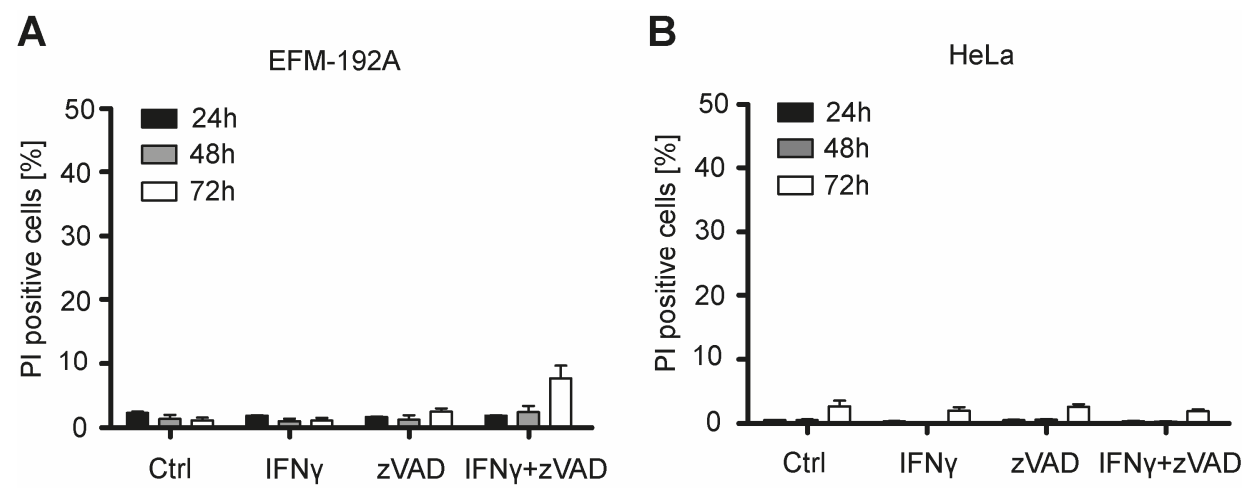

Figure 9. IFN $\gamma$ and $\mathrm{zVAD}$.fmk treatment for different periods of time led to cell death in EFM-192A cell. EFM-192 (A) or HeLa (B) cells were treated with IFN $\gamma$ and/or zVAD.fmk for 24, 48 or 72 hours. Cell death was observed by counting PI positive cells. Data are shown as mean + SEM. $n=3$.

Since IFNs are able to reduce cell viablitiy, we treated EFM-192A and HeLa cells with IFN $\gamma$ and zVAD.fmk for 24, 48 and 72 hours and performed MTT assay (Figure 10). The MTT assay is a colorimetric assay to determine cell meatbolism activity, which is an indicator for cell viability, proliferation and cytotoxicity. During this assay a yellow tetrazolium salt (MTT) is added to the cells and NAD(P)H-dependent oxidoreductase enzymes of viable cells reduce the salt to purple formazan crystals, which is determined by measuring the absorbance [55]. The combination treatment reduced significantly the cell viability of EFM-192A cells at all time points (Figure 10 A). In HeLa cells, both IFN $\gamma, z V A D$.fmk and the combination reduced cell viability at 48 and 72 hours (Figure $10 \mathrm{~B})$.

A

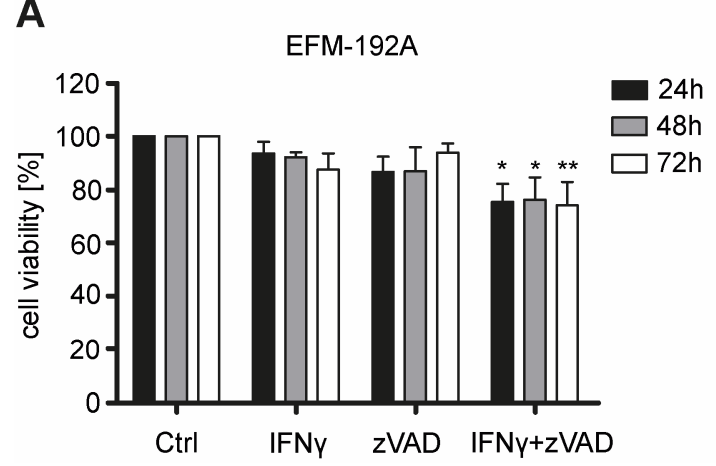

B

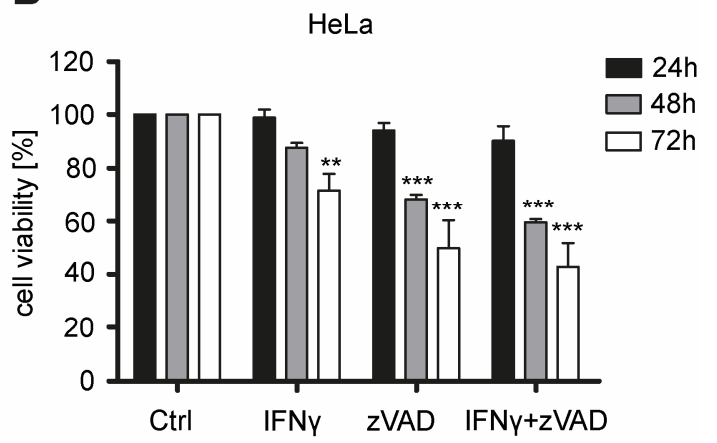

Figure 10. IFN $\gamma$ and zVAD.fmk reduced cell viability in EFM-192A and HeLa cells. EFM-192A (A) or HeLa (B) cells were treated with IFN $\gamma$ and/or zVAD.fmk for 24,47 or 72 hours and cell viability was analyzed via MTT assay. Data are normalized to untreated control (Ctrl) and are shown as mean + SEM. $n=3-4$. 
To get more insights into the type of cell death IFN $\gamma$ is inducing, phosphorylation of MLKL and inhibition of MLKL by NSA were studied (Figure 11). IFN $\gamma$ was able to induce slighlty phosphorylation of MLKL (pMLKL; Figure 11 A) and cell death was slighlty inhibited by NSA (Figure 11 B). This indicated that IFN $\gamma$ together with zVAD.fmk induced necroptosis in EFM-192A cells. Interestingly, treatment with IFN $\gamma$ induced higher protein levels of MLKL (Figure $11 \mathrm{~A}$ ). This upregulation of MLKL after IFN $\gamma$ treatment was a completely new finding and was studied further.

A

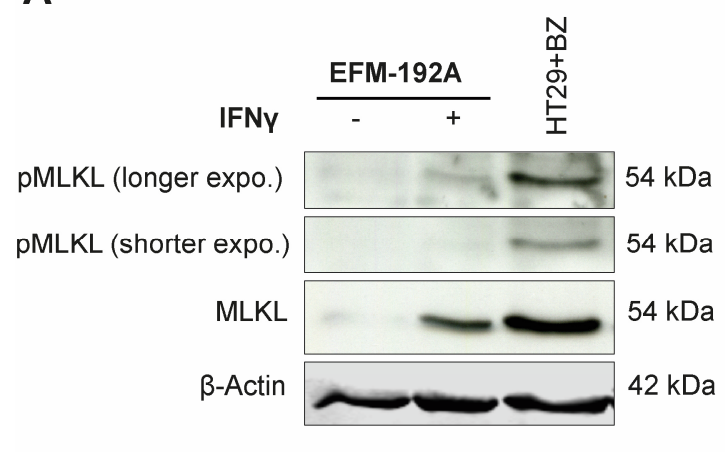

B

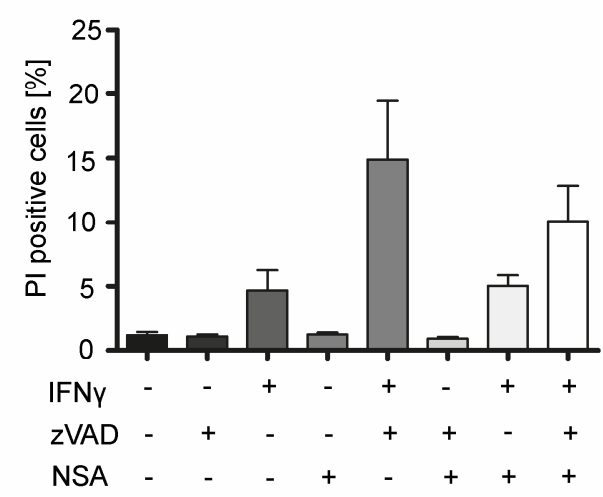

Figure 11. IFN $\gamma$ induced necroptosis in EFM-192A cells. A. EFM-192A cells were treated with IFN $\gamma$ for 72 hours and protein levels of pMLKL, MLKL and $\beta$-Actin were analyzed via Western Blotting. As control for phosphorylation of MLKL, HT29 cells were treated with the Smac-mimetic BV6 and zVAD.fmk (BZ). B. EFM-192A cells were treated with IFN $\beta$, zVAD.fmk and/or NSA for 72 hours and cell death was observed by counting PI positive cells. Data are shown as mean + SEM. $n=3$.

4.1.2. IFNs increase MLKL mRNA and protein levels in different cancer cell lines over time

To study the mechanisms underlying the IFN $\gamma$-induced upregulation of MLKL, EMF192A and HeLa cells were treated with IFN $\gamma$ for different time points and mRNA and protein levels of the necroptosis signaling protein MLKL and IRF1 were anaylzed. IFN $\gamma$ treatment increased mRNA level of MLKL and IRF1 in EFM-192A and HeLa cells significantly (Figure 12 A-D). MLKL protein level was increased in both cell lines, too (Figure $12 \mathrm{E}$ and F). Simultaneously with MLKL, protein levels of IRF1 and STAT1 were elevated, as well as STAT1 was phosphorylated. In summary, activation of the IFN $\gamma$ pathway and increased levels of MLKL were shown at the same time. 
A

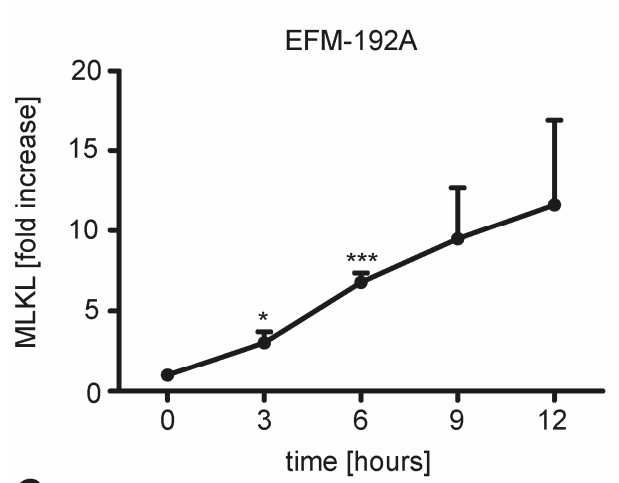

C

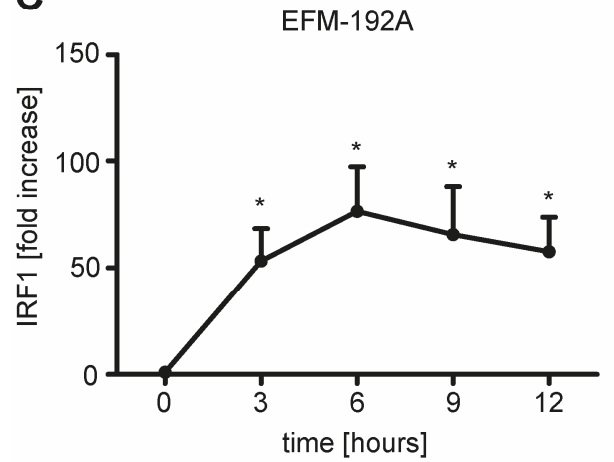

E

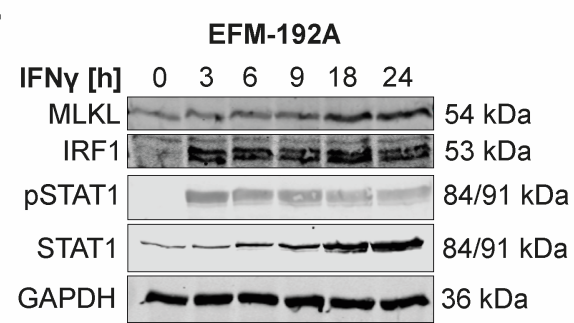

B

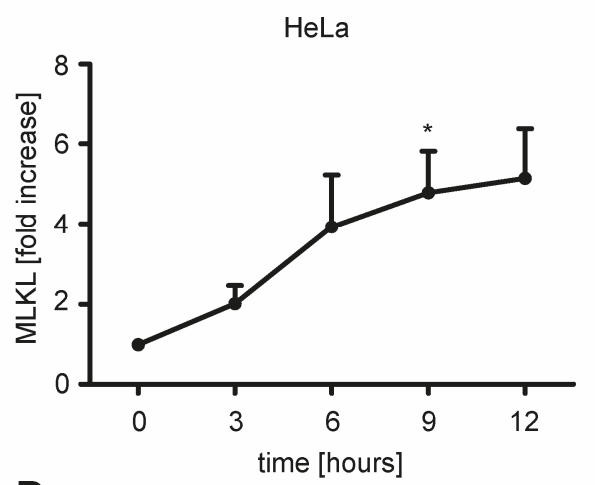

D

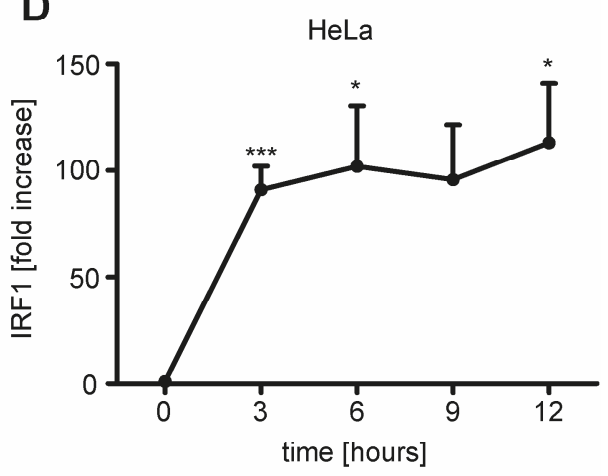

$\mathbf{F}$

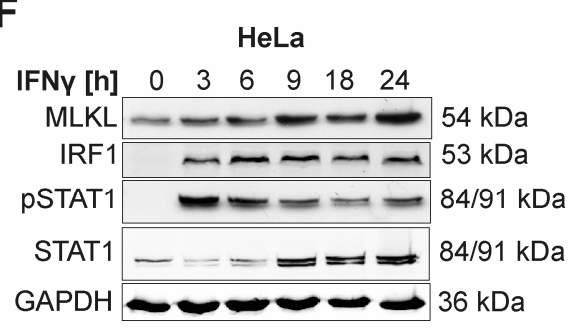

Figure 12. IFN $\gamma$ treatment increased MLKL and IRF1 mRNA and protein level over time. Cells were treated with $1.5 \mathrm{ng} / \mathrm{ml}$ (EFM-192A) or $10 \mathrm{ng} / \mathrm{ml} \mathrm{IFN \gamma}$ (HeLa) for different periods of time. A.-D. mRNA was isolated and fold increase of MLKL or IRF1 relative to untreated control are shown as mean + SEM. $\mathrm{n}=3-5$. E. + F. Protein expression of MLKL, IRF1, pSTAT1, STAT1 and GAPDH were anaylzed via Western blotting.

Next, we wanted to know wether other types of IFNs could also induce MLKL expression and this could be reproduced in EFM-192A, HeLa and other cell lines. Therefore, EFM192A, HeLa, MDA-MB 231 and MV4-11 cells were treated with IFN $\alpha$, IFN $\beta$ or IFN $\gamma$ (Figure 13). Similar to treatment with IFN $\gamma$, treatment with type 1 IFNs $\alpha$ and $\beta$, respectively, lead to increased MLKL protein level in all tested cell lines (Figure 13 A, C, E and F). Densitometric analysis of three different Western Blots of EFM-192A and HeLa cells revealed significantly increased MLKL protein level after treatment with all IFNs (Figure $13 \mathrm{~B}$ and D). 
A

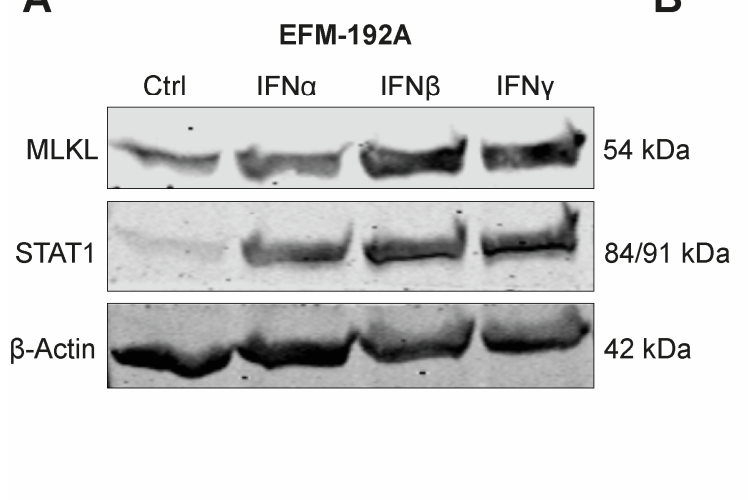

C

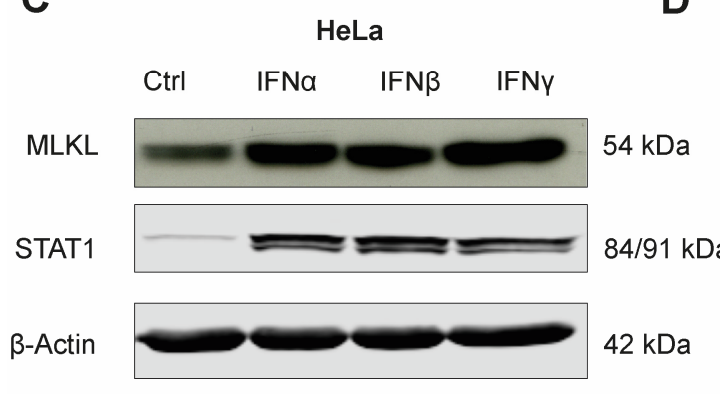

E

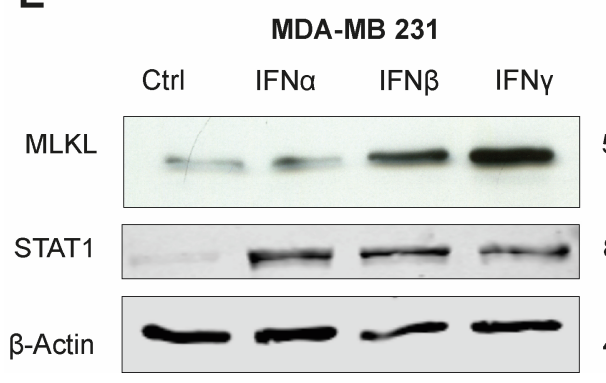

B

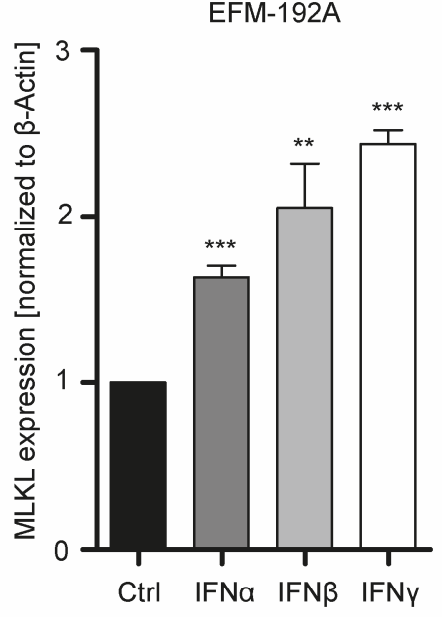

D

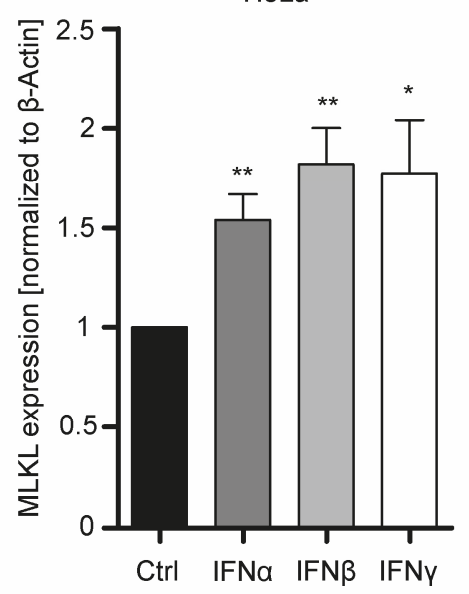

Figure 13. Type 1 and 2 IFNs increased expression of MLKL in different cell lines. A, C, E and $F$ EFM-192A, HeLa, MDA-MB 231 or MV4-11 cells were treated with IFN $\alpha, \beta$ or $\gamma$ for 72 hours and protein expression of MLKL, STAT1 and $\beta$-Actin were analyzed via Western Blotting. B+D Three Western Blots were analyzed via densitometry and MLKL expression normalized to unstimulated control is shown as mean + SEM. $n=3$.

To analyze if other members of the necroptosis pathway are regulated by IFN treatments as well, mRNA levels of RIPK1 and RIPK3 were studied in EFM-192A and HeLa cells (Figure 14). Treatment with IFN $\gamma$ led to slightly increased mRNA levels of RIPK1 and RIPK3 in EFM-192A cells and RIPK1 in HeLa cells, which do not express RIPK3 due to genomic methylation near the RIPK3 transcription start site [56]. Upregulation of RIPK1 
and RIPK 3 could not be confirmed on protein level (data not shown), indicating that IFNs do not regulate expression of other necroptotic proteins than MLKL.
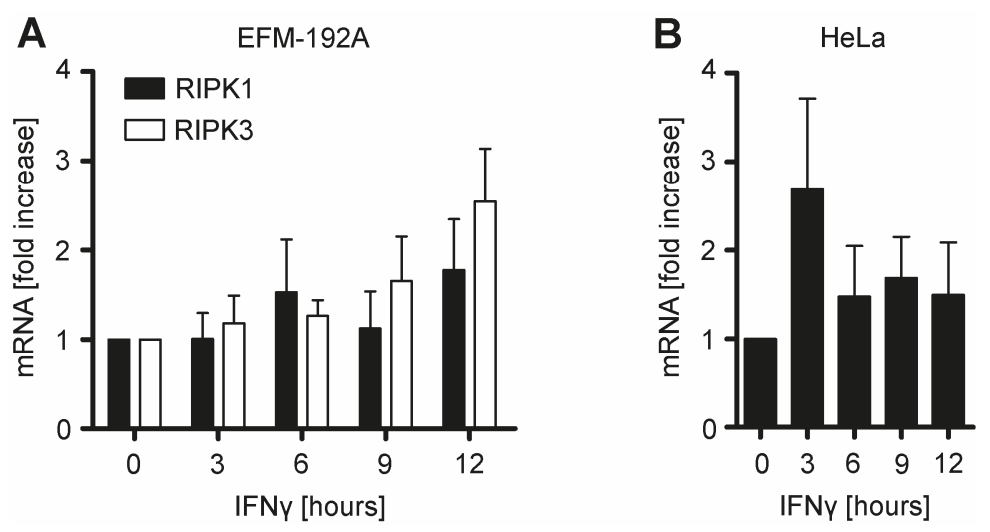

Figure 14. mRNA level of RIPK1 and RIPK3 after IFN $\gamma$ treatment. A. EFM-192A cells were treated with IFN $\gamma$ for indicated time points and mRNA of RIPK1 and RIPK3 fold increase normalized to untreated control are shown as mean \pm SEM. $n=3-4$. B. HeLa cells were treated with IFN $\gamma$ for indicated time points and mRNA of RIPK1 fold increase normalized to untreated control are shown as mean + SEM. n=4-5.

\subsubsection{Inhibition of transcription prevents IFN $\gamma$-induced MLKL expression}

To analyze if IFN-mediated MLKL upregulation is caused by increased transcription, an Actinomycin D chase experiment was performed (Figure 15). Actinomycin D is able to inhibit transcription by binding to DNA and thereby preventing the unwinding of the DNA double helix. As a result, the activity of the DNA-dependent RNA polymerase is blocked [57]. Inhibition of mRNA synthesis through Actinomycin D prevented the increase of MLKL protein level (Figure $15 \mathrm{~A}$ ) and significantly inhibited the increase of MLKL and IRF1 mRNA levels (Figure $15 \mathrm{~B}$ and $\mathrm{C}$ ), indicating that transcription of MLKL is induced by IFN $\gamma$ treatment. 

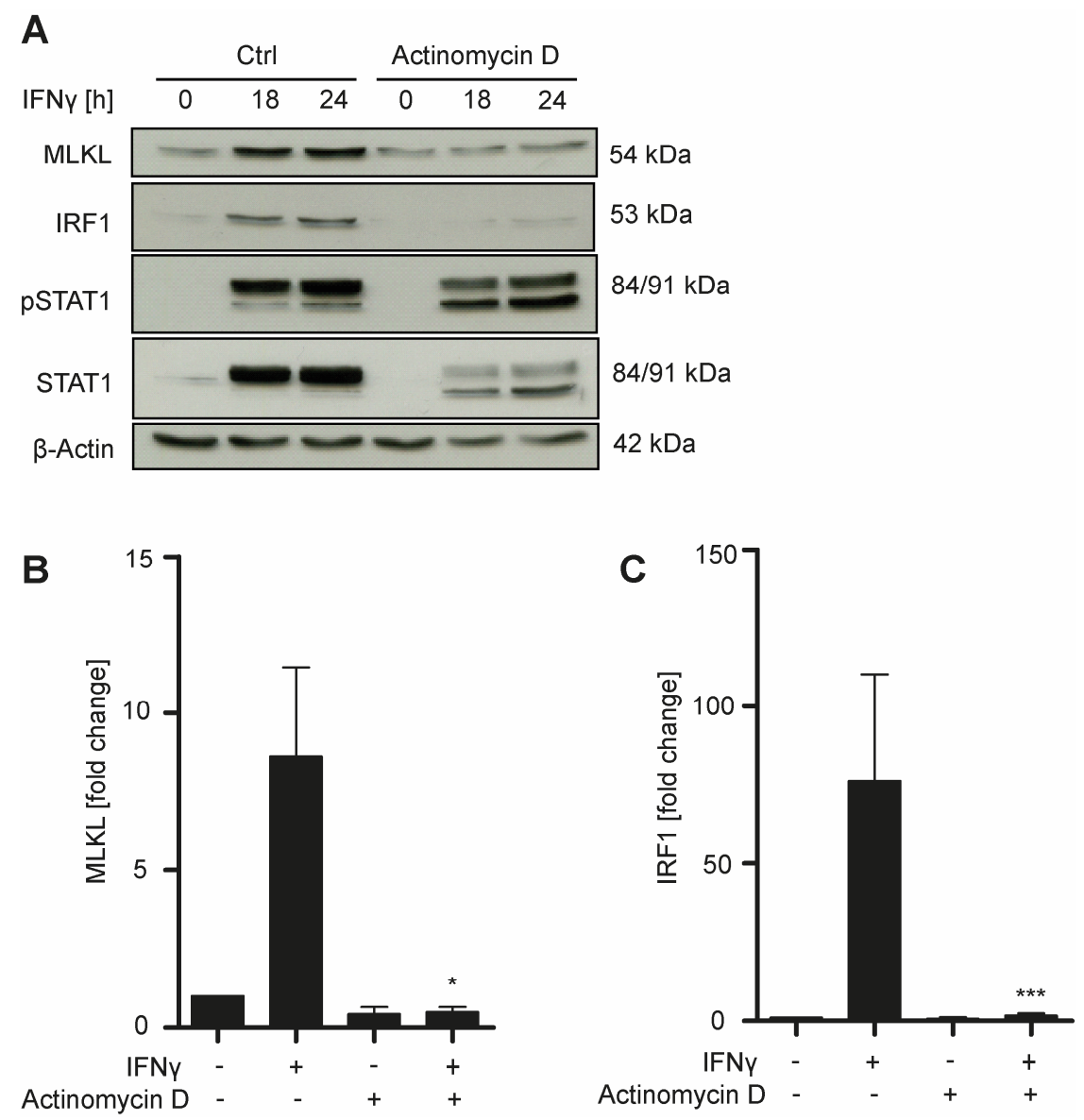

Figure 15. Inhibition of transcription by Actinomycin D inhibts MLKL upregulation. EFM-192A cells were treated for indicated time points with IFN $\gamma$ after 2 hours of Actinomycin D pre-treatment. A. Protein level of MLKL, IRF1, pSTAT1, STAT1 and $\beta$-Actin were analyzed via Western blotting. B.+C. Fold change of mRNA of MLKL and IRF1 normalized to untreated control are shown as mean + SEM. $n=4$.

\subsubsection{MLKL upregulation is indepent of activation of caspases}

IFNs are also known to induce and activate caspases [58]. To varify that IFN $\gamma$-dependent upregulation of MLKL was not influenced by elevated expression and activation of caspases, EFM-192A cells were treated with the pan-caspase inhibitor zVAD.fmk and IFN $\gamma$ (Figure 16). Inhibition of caspases did not effect the IFN $\gamma$-induced upregulation of MLKL. 


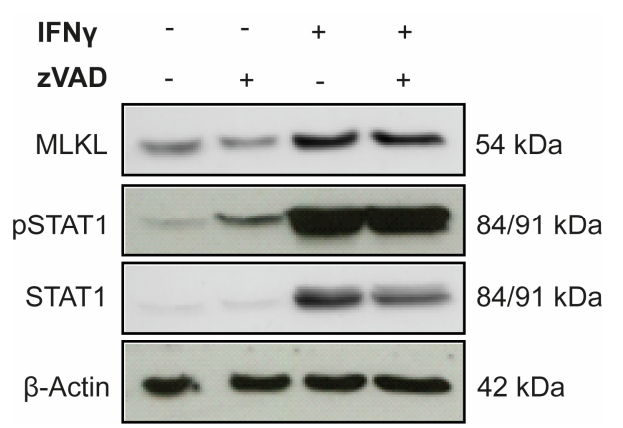

Figure 16. Inhibition of caspases had no effect on IFN $\gamma$-dependent upregulation of MLKL. EFM192A cells were treated with IFN $\gamma$ and zVAD.fmk. 24 hours after treatment, protein level of MLKL, pSTAT1, STAT1 and $\beta$-Actin were analyzed via Western Blotting.

\subsubsection{IRF1 and STAT1 contribute to MLKL up-regulation by IFN $\gamma$}

Since IFN $\gamma$ induced also IRF1 upregulation on mRNA and protein level, we wanted to confirm the contribution of the transcription factor IRF1 in upregulating MLKL. We performed a prediction analysis using the Eukaryotic Promoter Database and could confirm, that the promoter of MLKL contains STAT1 and IRF1 binding sites. Additionally, the platform Interferome was used to search for published datasets of IFNregulated gene sets. MLKL was found to be upregulated more than 2-fold in 71 datasets (Table 23 in appendix).

Furthermore, EFM-192A cells were transiently transfected with two distinct siRNAs targeting IRF1 or non-silencing siRNA (Figure 17). 72 hours after knockdown, cells were treated with IFN $\gamma$ for 24 hours, as IRF1 protein level were only detectable after IFN pathway activation. Efficient knockdown of IRF1 was confirmed on protein and mRNA level (Figure 17 A and C). Knockdown of IRF1 did not effect the upregulation of MLKL on protein and mRNA level (Figure $17 \mathrm{~A}$ and $\mathrm{B}$ ). 

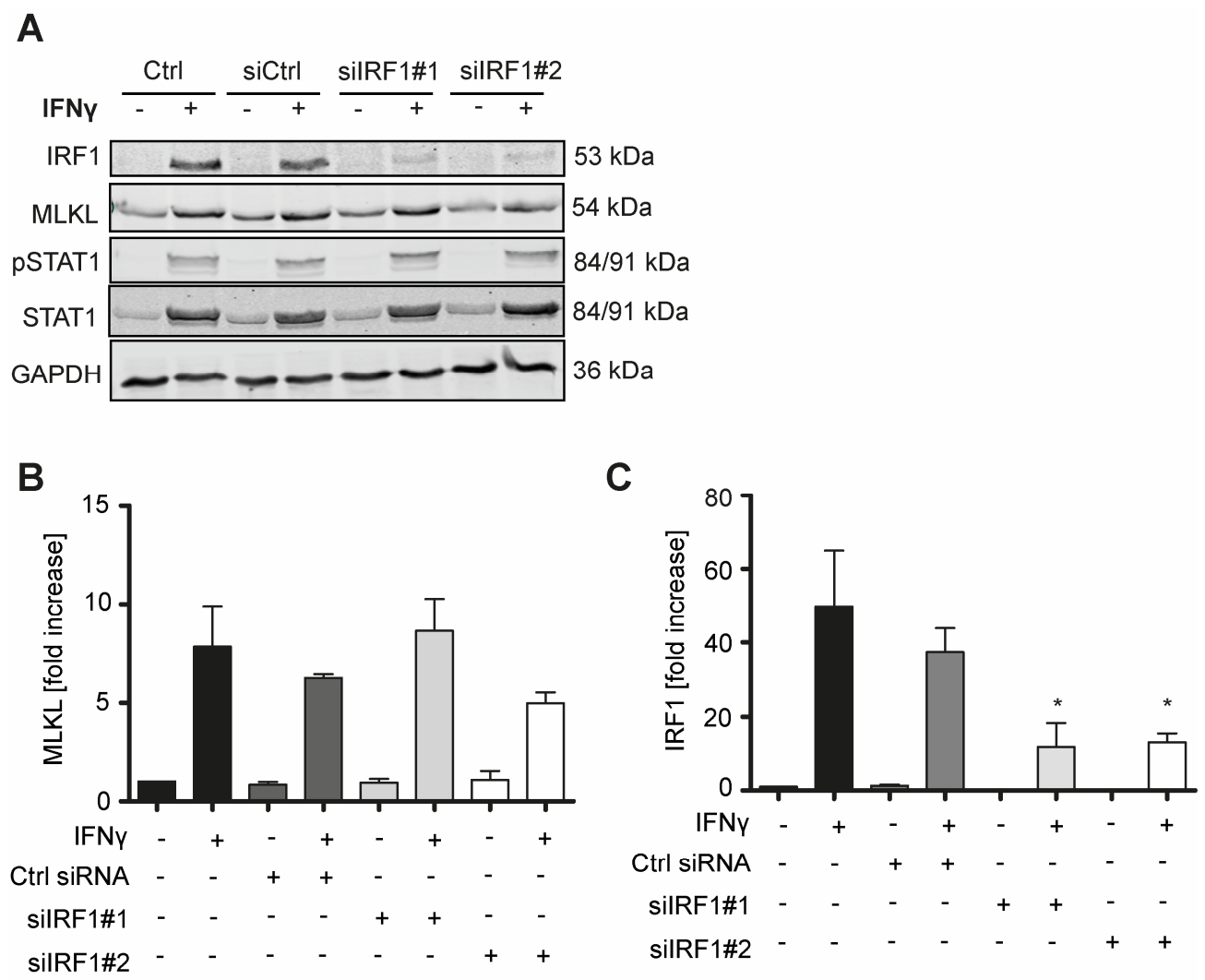

Figure 17. Single knockdown of IRF1 had no effect on MLKL upregulation. EFM-192A cells were transciently transfected with two distinct siRNAs targeting IRF1. 72 hours after knockdown cells were treated with IFN $\gamma$ for 24 (A) or 9 hours $(B+C)$. A. Protein expression of IRF1, MLKL, pSTAT1, STAT1 and GAPDH were analyzed using Western blotting. B.+C. Fold increase of MLKL and IRF1 mRNA levels are shown as mean + SEM. $n=4$.

Next, we wanted to interrupt the IFN pathway at an earlier step and performed a STAT1 knockdown by transiently transfection of EFM-192A cells with two distinct siRNAs targeting STAT1 (Figure 18). To analyze the effect on MLKL upregulation, cells were again treated with IFN $\gamma$ after knockdown. The single STAT1 knockdown was able to reduce STAT protein (Figure $18 \mathrm{~A}$ ) and IRF1 on mRNA level (Figure $18 \mathrm{C}$ ), but had no significant effect on MLKL upregulation, neither on protein nor at mRNA level (Figure $18 \mathrm{~A}$ and $\mathrm{B})$. 

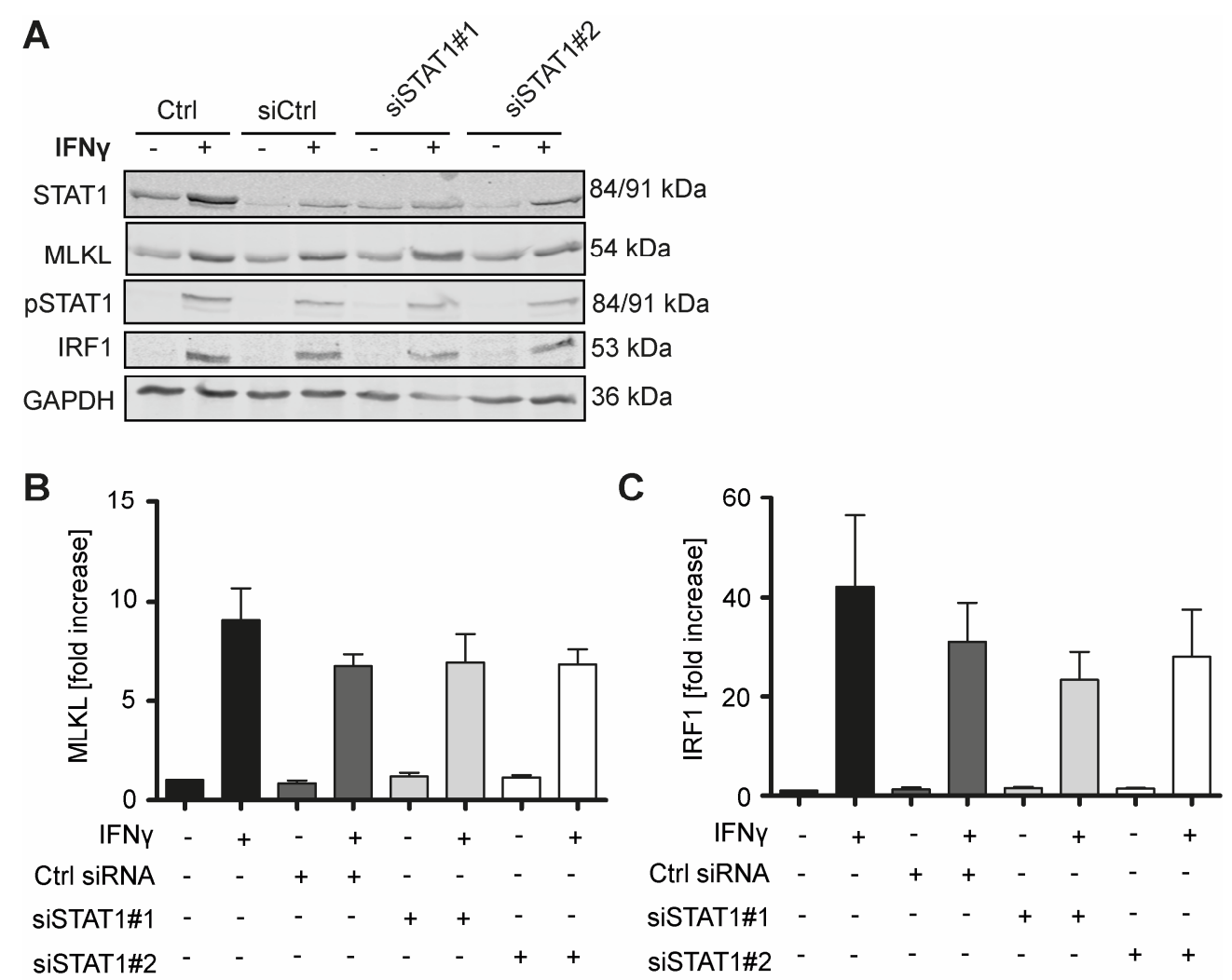

Figure 18. Single knockdown of STAT1 had no effect on MLKL upregulation. EFM-192A cells were transciently transfected with two distinct siRNAs targeting STAT1. 72 hours after knockdown cells were treated with IFN $\gamma$ for 24 (A) or 9 hours $(B+C)$. A. Protein expression of STAT1, MLKL, pSTAT1, IRF1 and GAPDH were analyzed using Western blotting. B.+C. Fold increase of MLKL and IRF1 mRNA levels are shown as mean + SEM. $n=5$.

As both single knockdowns of IRF1 and STAT1 had only slightly effects on the upregulation of MLKL, we knocked down IRF1 and STAT1 simultaneously by transiently transfecting EFM-192A cells with siRNA targeting IRF1 and siRNA targeting STAT1 (Figure 19). The double knockdown prevented the IFN $\gamma$-induced upregulation of MLKL on protein level (Figure 19 A) and significantly on mRNA level (Figure 19 B) for all different siRNAs. Efficient knockdown was shown by significantly reduced mRNA level of IRF1 (Figure 19 C) and reduced protein levels of IRF1 and STAT1 (Figure 19 A). These findings indicate that transcription factors IRF1 and STAT1 are necessary to upregulate MLKL expression after stimulus with IFN $\gamma$. 


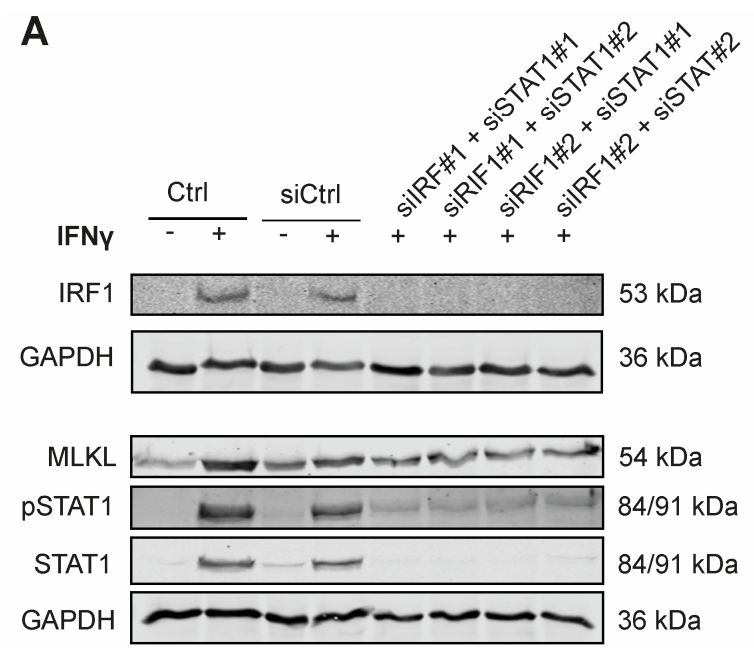

B

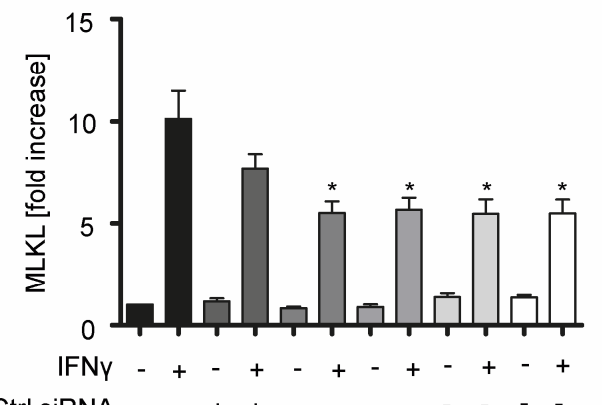

C

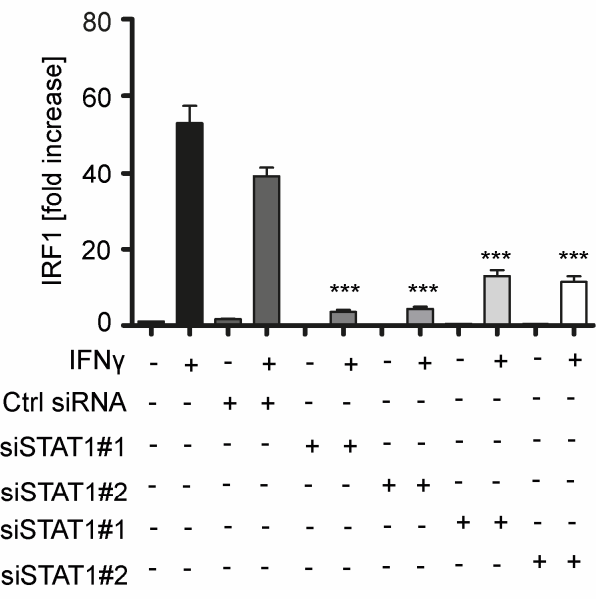

Figure 19. Double knockdown of IRF1 and STAT1 abolished MLKL upregulation. EFM-192A cells were transciently transfected with two distinct siRNAs targeting IRF1 and STAT1, respectively. 72 hours after knockdown cells were treated with IFN $\gamma$ for 24 (A) or 9 hours $(B+C)$. A. Protein expression of IRF1, MLKL, pSTAT1, STAT1 and GAPDH were analyzed using Western blotting. B.+C. Fold increase of MLKL and IRF1 mRNA levels are shown as mean + SEM. $\mathrm{n}=7$.

Finally, we used CRISPR/Cas9 technology to create IRF1 knockout MBA-MD-231 cells. Efficient IRF1 knockout was confirmed by Western Blotting (Figure 20). IFN $\gamma$ treatment in IRF1 knockout cells did not lead to MLKL upregulation, suggesting that IRF1 is important for MLKL upregulation. 


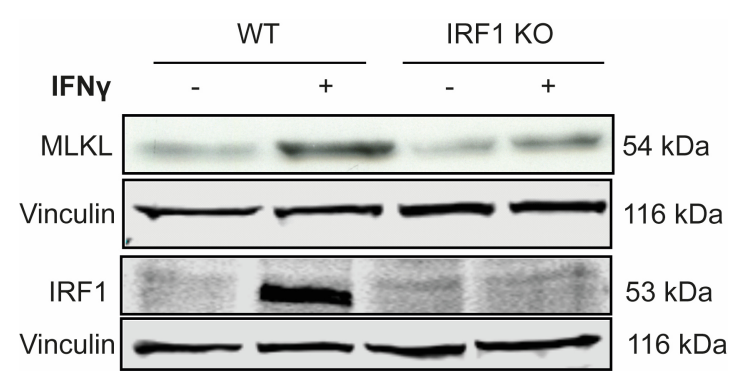

Figure 20. Knockout of IRF1 prevented IFN $\boldsymbol{\gamma}$-induced MLKL upregulation. MBA-MD-231 IRF1 knockout cells were generated using CRISPR/Cas9. Protein expression of MLKL, IRF1, MLKL and Vinculin were analyzed using Western blotting. 


\subsection{Part II: Effect of dying cells on immune cells and resolution of inflammation}

Most of the current studies use biological or chemical substances to induce and study cell death. Thereby, also other pathways than programmed cell death are activated and could compromise the results. Especially the effect on studied immune cells could be influenced. Therefore, we used an inducible cell death system exclusively activating either apoptosis or necroptosis to study the effect of dying cells on immune cells with a focus on macrophages.

\subsubsection{Generation of inducible cell death systems}

We generated inducible systems of cell death, where only apoptosis or necroptosis are activated. To achieve this, a murine fibroblast cell line (NIH 3 T3 cells) stabil expressing either hCasp8 or mRIPK3 was created. Both hCasp8 and mRIPK3 were genetically modified and fused to one or two dimerizer domains (FV; Figure $21 \mathrm{~A}$ ). In the case of hCasp8, the N-terminal DEAD domain was replaced by the dimerizer domain. Two dimerizer domains were fused C-terminally to mRIPK3. The dimerizer will bind to these domains and is able to bring two proteins in close proximity, which leads to autophosphorylation and activation of hCasp8 or mRIPK3. Green fluorescent protein (GFP) was co-expressed in these cell as selection marker.

After transduction, single cells were seeded in 96 well plates to grow single clones. Protein expression of hCasp8 or mRIPK3 and GFP were analyzed via Western Blotting (Figure $21 \mathrm{~B}$ and C). Cells, which express both hCasp8 and GFP or mRIPK3 and GFP were selected (indicated with arrows) and treated with dimerizer to induce cell death (Table 22). Additionally, GFP-fluroescence intensity was measured using flow cytometry (Table 22). 
A

$\triangle \mathrm{N}-\mathrm{FV}$-hCaspase-8:

\begin{tabular}{|c|c|c|}
\hline FKBP & Large subunit & Small subunit \\
\hline mRIPK3-2xFV: & & \\
\hline kinase domaine & RHIM FKBP & FKBP \\
\hline
\end{tabular}

B

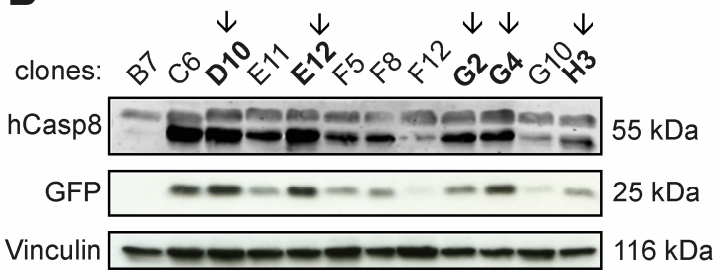

C

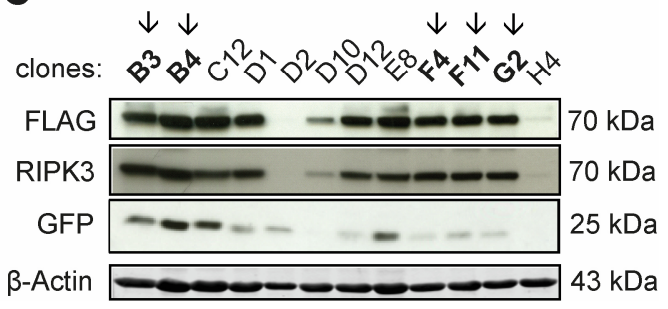

Figure 21. NIH 3T3 cells stabily expressing FV-hCasp8 or mRIPK3-FV were generated. A. To create a inducible apoptotic system, the DEAD domain of human caspase 8 was replaced by a dimerizer domain (FKBP or FV). An inducible necroptotic cell death system was created by fusing two dimerizer domains to the C-terminus of murine RIPK3. Single clones were generated afer successful transduction. B. Protein expression of hCasp, GFP and Vinculin of NIH 3T3 cells after transduction with plasmids containg hCasp8FV was analyzed using Western Blotting. C. Protein expression of FLAG, RIPK3, GFP and $\beta$-Actin of NIH 3 T3 cells after transduction with plasmid containing mRIPK3-FV were analyzed via Western Blotting.

Table 22. Dimerizer-induced cell death and GFP fluorencence intensity of NIH 3T3 single clones were analyzed. NIH 3T3 FV-hCasp8 or mRIPK3-FV cells were treated with dimerizer for 18 or 4 hours and PI positive cells were counted. Data are shown as mean. GFP fluorescence intensity was measured using flow cytometry and is shown as relative fluorescence unit (RFU).

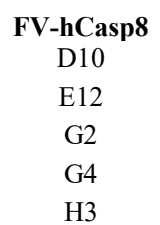

mRIPK3-FV

B3

B4

F4

F11

G2

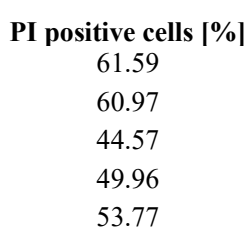

PI positive cells [\%]

63.86

60.34

58.84

53.73

67.44
GFP fluorescence (RFU)

3707

3637

3243

5315

3006

GFP fluorescence (RFU)

4427

2922

3176

1493

1391

For the apoptotic contruct, we selected clone D10 and E12 based on the hCasp8 and GFP protein expression, GFP fluroescence intensity and induction of cell death at around $60 \%$ after 18 hours. Clone B3 and F11 were selected for the necroptotic construct, again based on mRIPK3 and GFP protein expression, GFP fluroescence intensity and cell death induction measured after 4 hours (between 50 and $60 \%$ ). Protein expression was also confirmed at later passages (Figure 22). 


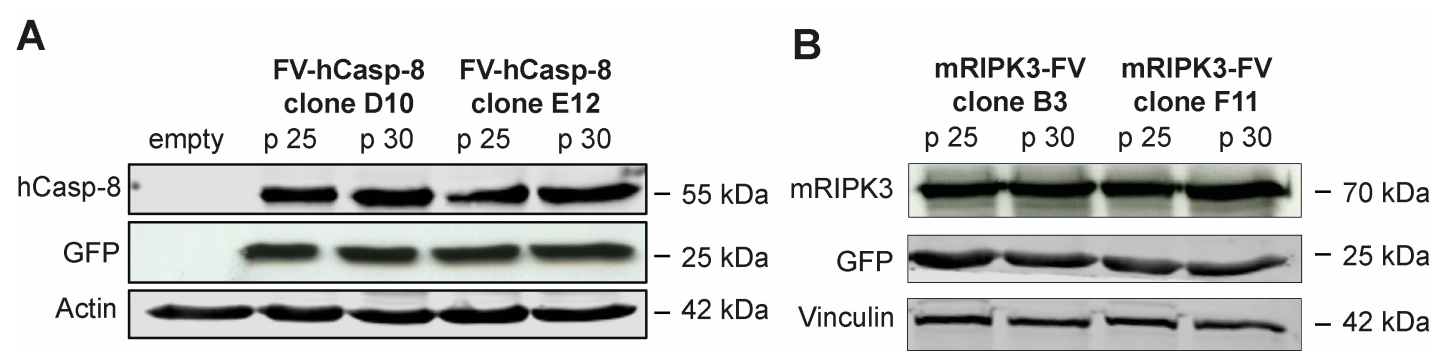

Figure 22. Expression of FV-hCasp8 and mRIPK3-FV was confirmed at higher passages. A. Protein expression of hCasp8, GFP and $\beta$-Actin in NIH 3T3 empty cells and FV-hCasp8 clones D10 and E12 were analyzed via Western Blotting after 25 and 30 passages (p). B. Protein expression of mRIPK3, GFP and Vinculin in NIH 3T3 mRIPK3-FV clones B3 and F11 were analyzed via Western Blotting after 25 and 30 passages (p).

To determine the optimal concentration of dimerizer, which induces the highest percentage of dying cells, a dose-response experiment was performed (Figure 23). Cells were treated with 0 to $250 \mathrm{nM}$ dimerizer for 18 (FV-hCasp8, Figure $23 \mathrm{~A}$ ) or 4 hours (mRIPK3-FV, Figure 23 B). In both cell lines, concentrations starting from $5 \mathrm{nM}$ dimerizer induced high levels of cell death and could be inhibited by either pan-caspase inhibitor zVAD.fmk or RIPK3 inhibitor GSK'872, respectively, indicating that dimerizer treatment induced apoptosis in FV-hCasp8 and necroptosis in mRIPK3-FV cells, as expected. For further experiments we chose a concentration of $10 \mathrm{nM}$ dimerizer.

A

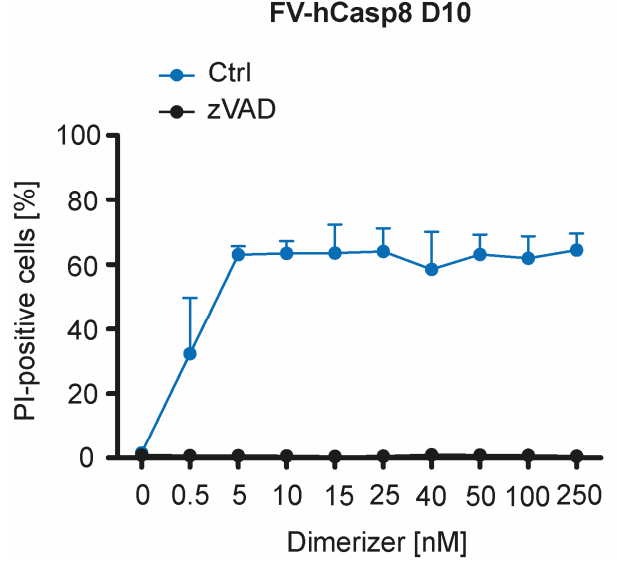

B

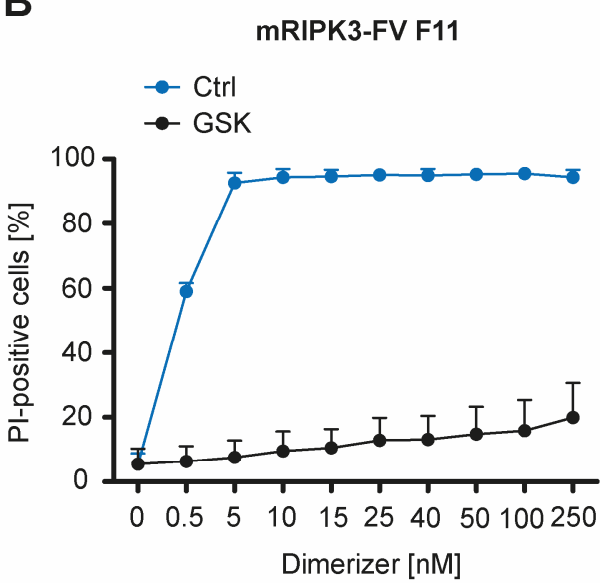

Figure 23. Optimal concentration of dimerizer was tested by measuring cell death in a dose range of dimerizer. A. FV-hCasp8 cells were treated with zVAD.fmk and/or indicated concentrations of dimerizer for 18 hours and PI positive cells were counted. B. mRIPK3-FV cells were treated with GSK' 872 and/or indicated concentrations of dimerizer for 4 hours and PI positive cells were counted. Data are shown as mean + SEM. $n=3$. 
To determine the optimal treatment length, cells were treated for different periods of time with $10 \mathrm{nM}$ dimerizer (Figure 24). High percentages of PI-positive FV-hCasp8 cells were observed between 18 and 48 hours (Figure 24 A), whereas mRIPK3-FV cells started to reach high percentages after 4 hours (Figure 24 B). Wild type (empty) NIH 3T3 cells were not dying upon dimerizer treatment. For further experiments FV-hCasp8 cells were treated for 18 hours and mRIPK-FV cells for 4 hours with dimerizer.
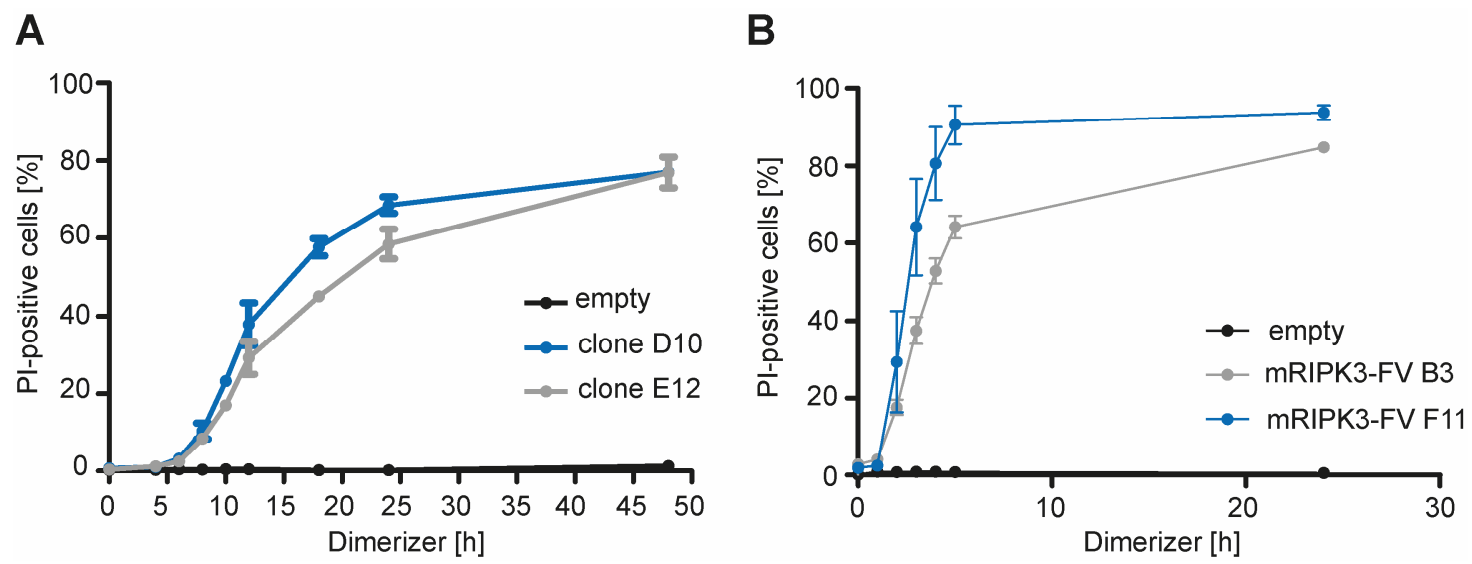

Figure 24. Optimal time point to measure dimerizer-induced cell death was determined. FV-hCasp8 (A.) or mRIPK3-FV cells (B.) were treated for indicated periods of time with $10 \mathrm{nM}$ dimerizer and PI positive cells were counted. Data are shown as mean \pm SEM. $n=3$.

During apoptosis, activation of caspase 8 leads to activation of executioner caspase 3 and 7 and phosphatidylserine exposure at the cell surface. To confirm induction of apoptosis after dimerizer treatment in FV-hCasp8 cells, caspases were inhibited with pan-caspase inhibitor zVAD.fmk and exposure of PS was measured by staining of PS with Annexin$\mathrm{V}$ and in an additional experiment caspase 3 and 7 positive cells were counted (Figure 25). zVAD.fmk was able to inhibit the significant increase of PI-positive cells after dimerizer treatment (Figure $25 \mathrm{~A}$ ). After 8 hours most of the dying cells were Annexin$\mathrm{V}$ positive, meaning they exposed PS before overall cell membrane integrity was lost (indicated by PI staining; Figure 25 B). Finally, activation of caspase 3 and 7 could be blocked completely by zVAD.fmk (Figure $25 \mathrm{C}$ ). These experiments confirmed activation of apoptosis after dimerizer treatment in FV-hCasp8 cells. 
A

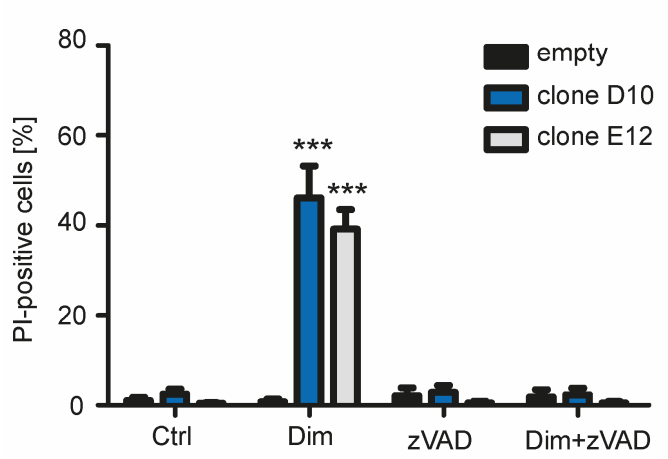

C

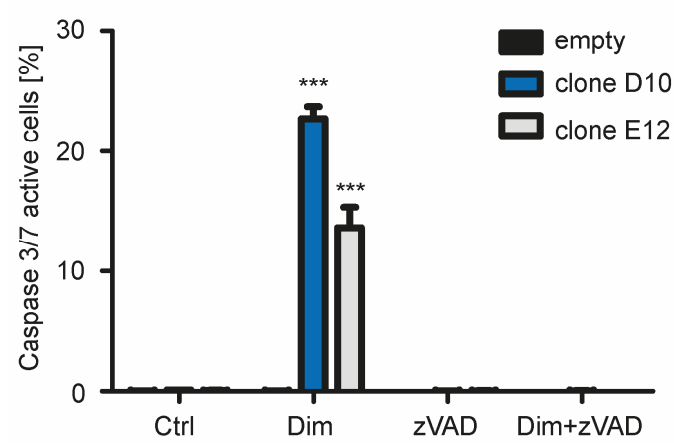

B

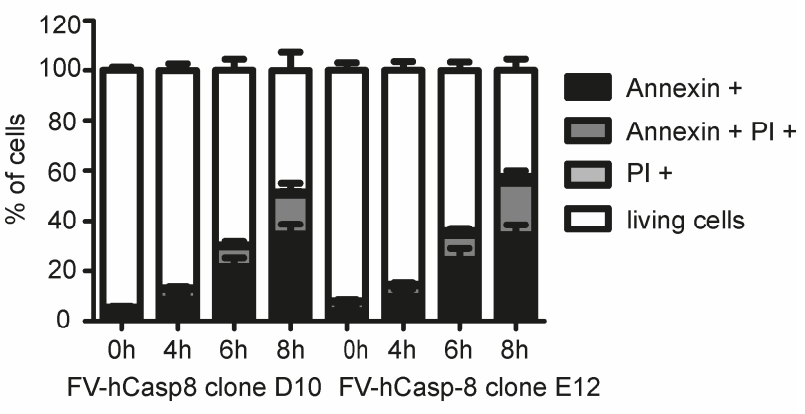

Figure 25. Dimerizer treatment induced apoptosis in FV-hCasp8 cells. A. FV-hCasp8 D10 or E12 or empty cells were treated with dimerizer and/or zVAD.fmk for 18 hours and PI positive cells were counted. $\mathrm{n}=3$. B. FV-hCasp8 D10 or E12 cells were treated with $10 \mathrm{nM}$ dimerizer for indicated time points. Annexin$\mathrm{V}$ and PI positive cells were measured using flow cytometry. $\mathrm{n}=4$. C. FV-hCasp8 D10 or E12 cells were treated with dimerizer and/or zVAD.fmk for 10 hours and caspase 3/7 active cells were counted. $n=3$. Data are shown as mean + SEM.

During necroptosis, RIPK1 and RIPK3 are activated and MLKL is phosphorylated. To confirm that dimerizer treatment of mRIPK3-FV cells leads to necroptosis, these cells were treated with dimerizer, RIPK3 inhibitor GSK'872 and/or RIPK1 inhibitor Nec-1s (Figure 26 A). Inhibiton of RIPK3 could completely block activation of cell death, whereas inhibition of RIPK1 only slightly reduced the amount of PI-positive cells. Furthermore, dimerizer treatment led to phosphorylation of MLKL within short time frames of about 30 minutes (Figure $26 \mathrm{~B}$ ). Taken together, dimerizer treatment induced the activation of RIPK3-FV, which leads to RIPK1-independent phosphorylation of MLKL. 
A

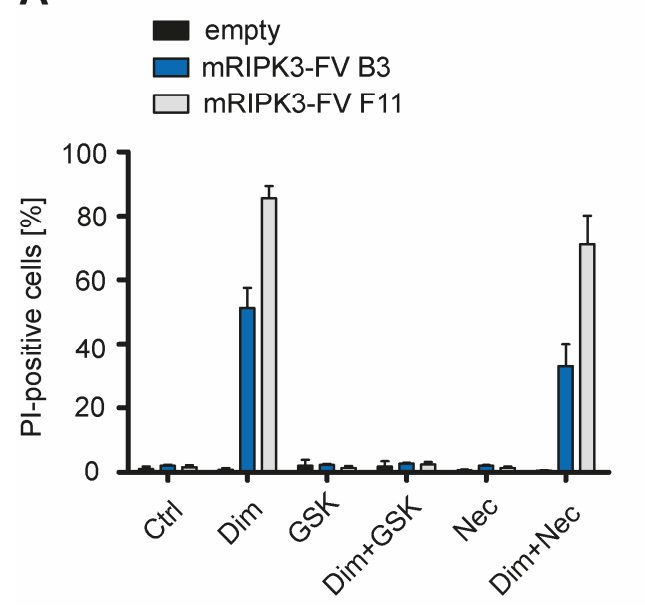

B

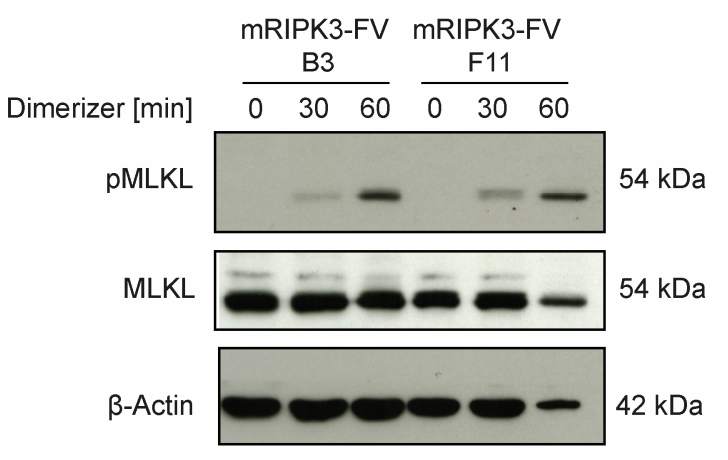

Figure 26. Dimerizer treatment induced necroptosis in mRIPK3-FV cells. A. mRIPK3-FV B3 or F11 cells were treated with dimerizer, GSK' 872 and/or Nec-1s for 4 hours and PI positive cells were counted. Data are shown as mean + SEM. $n=3$. B. mRIPK3-FV B3 or F11 cells were treated with dimerizer for 30 or 60 minutes and expression of pMLKL, MLKL and $\beta$-Actin was analyzed by Western Blotting.

4.2.2. Establishing of a co-culture system of dying cells and peritoneal macrophages

Next, we sutdied the effect of apoptotic vs. necroptotic cells on peritoneal macrophages. To achieve this, we estabilshed a co-culture of peritoneal macrophages and NIH 3T3 cells using a transwell system (Figure 27). Cells were isolated from the peritoneum of mice and seeded in 24 well plates, with adherent cells being defined as peritoneal macrophages. NIH 3 T3 cells were seeded in transwell inserts and co-culture was started 1 to 4 hours after seeding. After an over night co-culture, cell death was induced with dimerizer and 48 hours later cytokines and chemokines in the supernatant, and mRNA in macrophages were analyzed. 


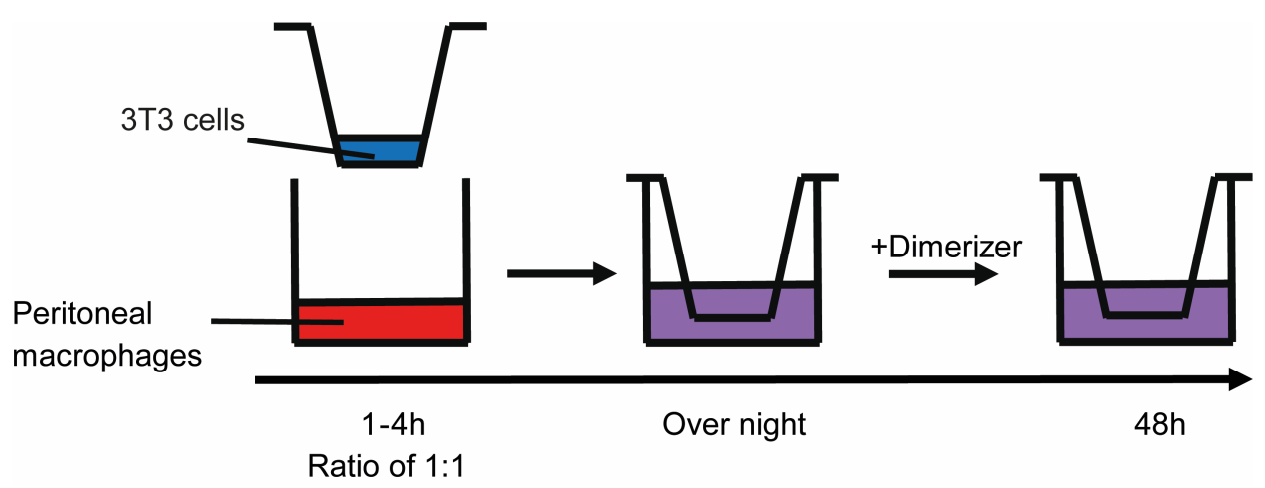

Figure 27. A co-culture system was estabilshed to study the effect of dying cells on peritoneal macrophages. Macrophages isolated from the peritoneum of mice were seeded in the bottom of 24 well plates and NIH 3T3 cells were seed in transwell inserts. 1 to 4 hours after seeding, cells were co-cultivated over night before dimerizer was added to induce cell death of the NIH 3 T3 cells. After 48 hours of coculture mRNA of macrophages and cytokines and chemokines in the supernatant were analyzed.

To test, whether macrophages are activated by dying cells in the co-culture system, expression of known macrophage polarization markers and released cytokines and chemokines by macrophages as response to dying cells were analyzed. The proinflammatory cytokine IL-6 is known to be released in response to necroptotic cells. In our setting, expression of IL-6 was significantly decreased in apoptotic FV-hCasp8 cells (Figure $28 \mathrm{~A}$ ) and increased in necroptotic mRIPK3-FV cells (Figure $28 \mathrm{~B}$ ). In both cell lines, inhibition of caspases or RIPK3 reversed the effect. IL-6 mRNA was also increased in macrophages after co-culture with necroptotic cells (Figure $28 \mathrm{C}$ ). IL-6 protein was released into the supernatant of necroptotic cells, but significantly higher levels of IL-6 were released during the co-culture of macrophages with necroptotic cells (Figure $28 \mathrm{D}$ ), indicating that both necroptotic cells and macrophages release IL-6 in the co-culture. 

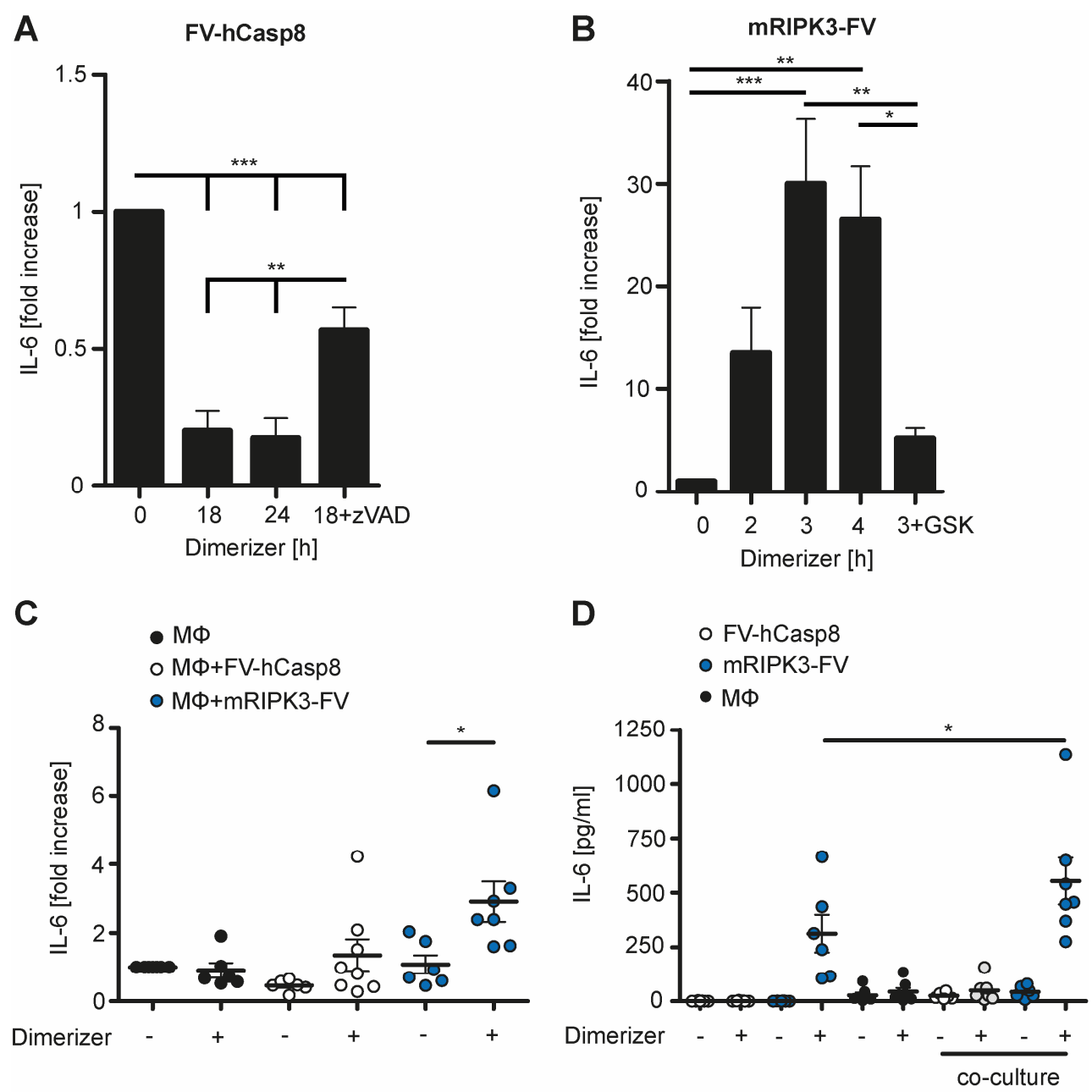

Figure 28. IL-6 is increased on mRNA and protein level in necroptotic cells and in macrophages cocultured with necroptotic cells. A. FV-hCasp8 D10 or E12 cells were treated with dimerizer and zVAD.fmk for indicated time points. mRNA was isolated and fold increase of IL- 6 normalized to control is shown as mean + SEM. $n=6-7$. B. mRIPK3-FV B3 or F11 cells were treated with dimerizer and GSK'872 for indicated time points. mRNA was isolated and fold increase of IL-6 normalized to control is shown as mean + SEM. $n=4-8$. C. Macrophages were co-cultured with FV-hCasp8 or mRIPK3-FV cells and cell death of NIH 3 T3 cells was induced with dimerizer. 48 hours after dimerizer treatment, mRNA of macrophages was isolated. Fold increase of IL-6 normalized to control is shown as mean \pm SEM. $\mathrm{n}=6-8$. D. NIH 3T3 cells and macrophages were cultured alone or in co-culture and cell death of NIH 3T3 cells was induced with dimerizer treatment. After 48 hours, IL-6 concentration in the supernatant was analyzed. Data are shown as mean \pm SEM. $n=6-7$.

To validate our co-culture system further, release of CXCL5 as response to apoptotic cells was analyzed as described previously [59]. Indeed, macrophages co-cultured with apoptotic cells showed increased concentrations of CXCL5 in the supernatant compared to untreated macrophages (Figure 29). 


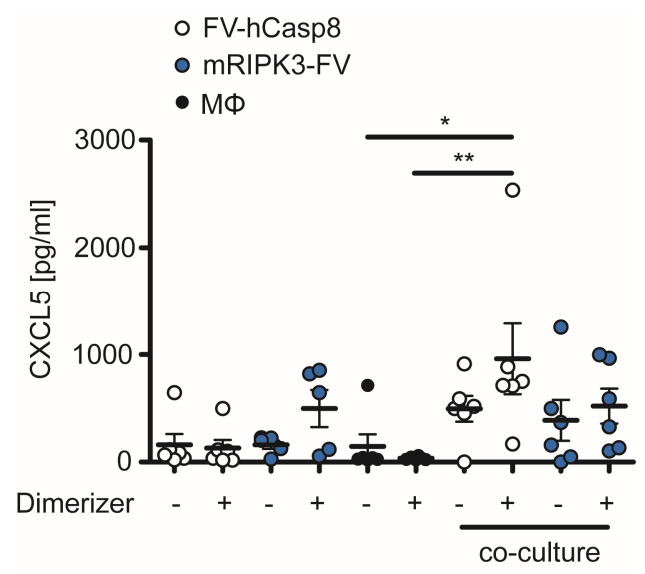

Figure 29. Co-culture of macrophages and apoptotic cells led to elevated levels of CXCL5 in the supernatant. NIH 3T3 cells and macrophages (MФ) were cultured alone or together and cell death of NIH 3T3 cells was induced with dimerizer. After 48 hours, $\mathrm{CXCl} 5$ concentration in the supernatant was analyzed. Data are shown as mean \pm SEM. $n=5-6$.

Finally, we analyzed mRNA levels of eight different pro- and anti-inflammatory macrophage polarization markers (TNF- $\alpha$, IL-1 $\beta$, IL-23, CCL2, CD206, ALOX15, Arg1, Ym-1). Differences in mRNA levels were seen for TNF- $\alpha$, Arg-1, CD206 and ALOX15 (Figure 24). mRNA levell of pro-inflammatory TNF- $\alpha$ were significantly reduced in macrophages co-cultured with necroptotic cells (Figure $30 \mathrm{~A}$ ), whereas anti-inflammatory Arg-1 mRNA level were slightly increased (Figure 30 B). Both mRNA level of antiinflammatory CD206 and ALOX15 were increased in macrophages co-cultured with NIH 3T3 cells, significantly increased in macrophages co-cultured with apoptotic cells and decreased again in macrophages co-cultured with necroptotic cells (Figure $30 \mathrm{C}$ and D). In summary, co-culture of dying NIH $3 \mathrm{~T} 3$ cells with macrophages using a transwell system was able to induce macrophage polarization, confirming that in this system macrophages are able to interact with dying cells. 

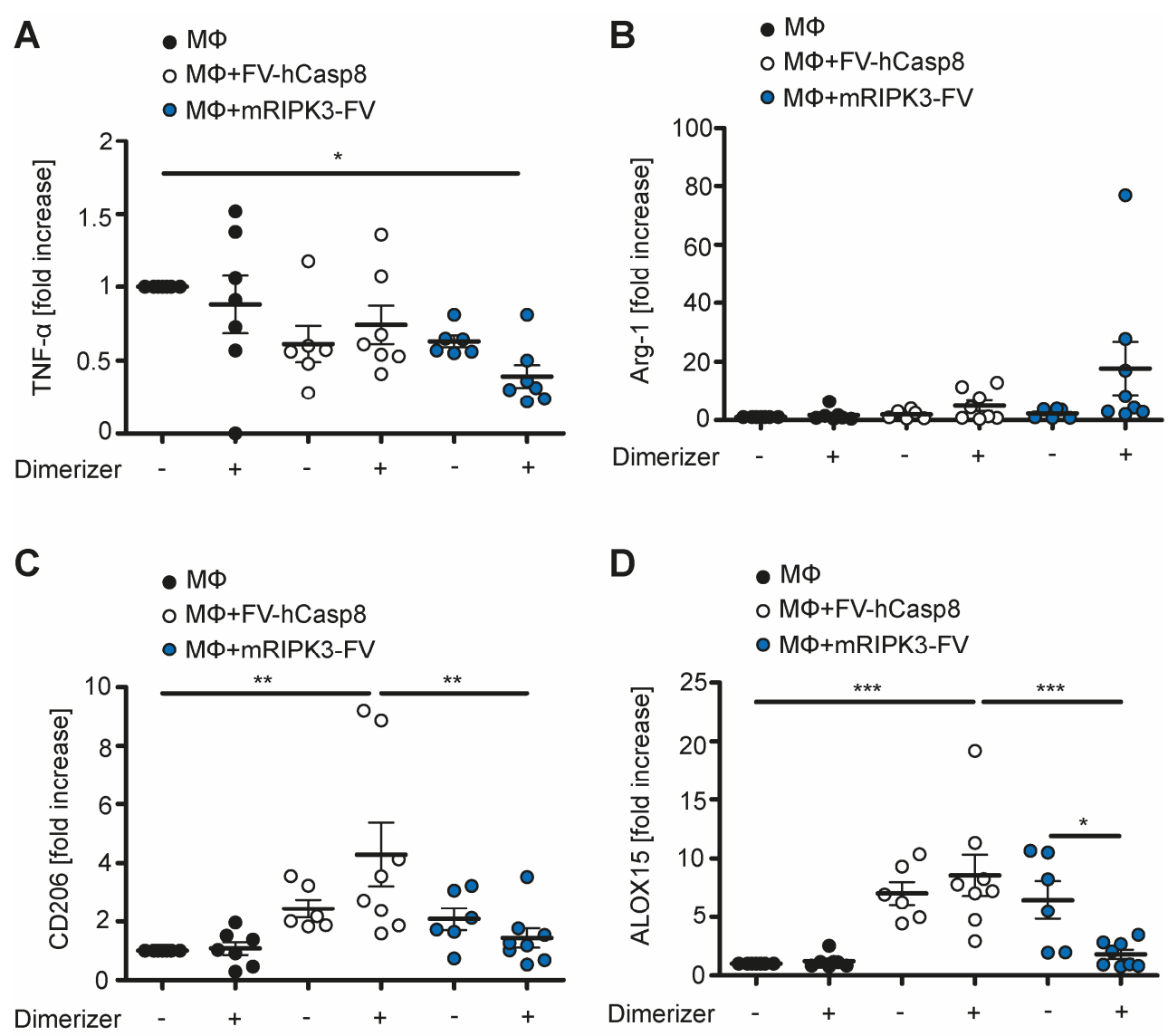

Figure 30. mRNA level of four different macrophage polarization marker were changed after coculture with dying NIH 3 T3 cells. A.-D. Macrophages were co-cultured with FV-hCasp8 or mRIPK3-FV cells and 48 hours after cell death induction through dimerizer treatment, mRNA of macrophages was analyzed. Fold increase of TNF- $\alpha$, Arg-1, CD206 and ALOX15 normalized to control are shown as mean \pm SEM. $\mathrm{n}=6-8$.

\subsubsection{RNA sequencing of macrophages co-cultured with dying cells}

To gain new insights into expression profiles of macrophages co-cultured with apoptotic or necroptotic cells, we used our estabilshed co-culture system and analyzed the macrophage transcriptome by whole genome mRNA sequencing. We selected clone D10 of FV-hCasp8 and clone F11 of mRIPK3-FV cells for further analysis. Peritoneal macrophages were either co-cultured with NIH $3 \mathrm{~T} 3$ cells and treated with dimerizer or were cultured with dimerizer alone as control. 48 hours after cultivation, mRNA of the macrophages was isolated and analyzed via mRNA sequencing (Figure 31). 


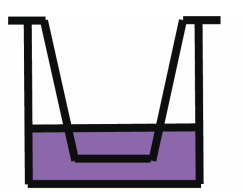

\section{$48 \mathrm{~h}$}

\section{1. $M \Phi+$ Dimerizer \\ 2. $M Ф+F V-C a s p 8+D i m e r i z e r$ \\ 3. MФ+mRIPK3-FV+Dimerizer}

Figure 31. The treatment procedure before RNA sequencing is depicted. Peritoneal macrophages (MФ) were co-cultivated with NIH $3 \mathrm{~T} 3$ cells and either apoptosis or necroptosis were induced by treatment with dimerizer. As control, macrophages were cultivated alone and treated with dimerizer. After 48 hours, mRNA of macrophages was isolated and mRNA sequencing was performed.

Differential gene expression from RNA sequencing data was analyzed by comparing all three treatment groups with each other. Principal component analysis (PCA) was performed to determine if the treatments were the major source of variation in gene expression. PCA plots showed a clear separation between all three sample groups, whereas the varitation between the four biological replicates was small (Figure 32).

A

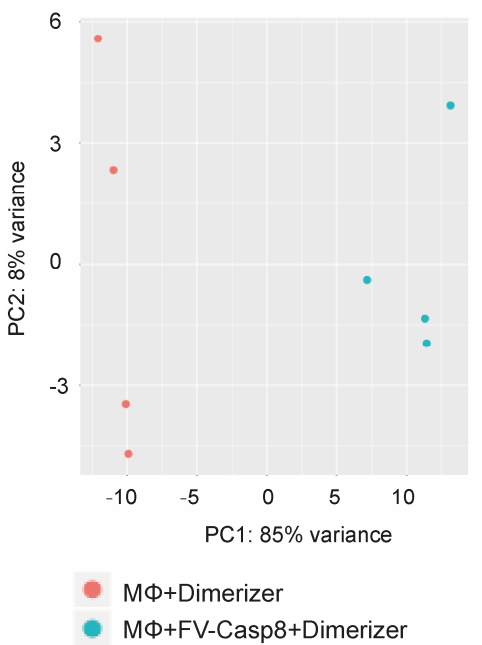

B

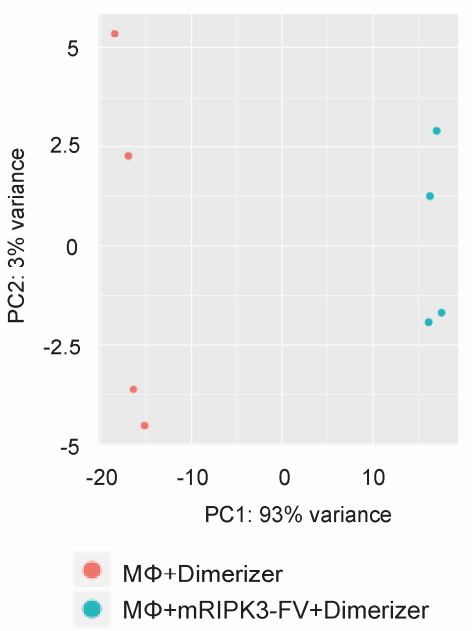

C

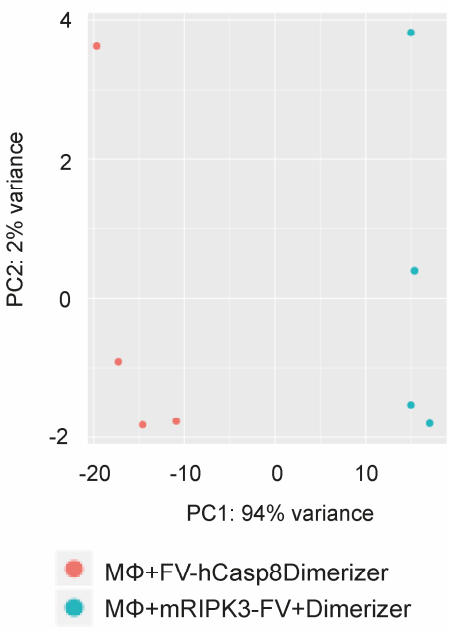

Figure 32. PCA plots demonstrated clear separation of treatment groups after RNA sequencing. PCA plots show variance of gene expression between sample groups. A. MФ+dimerizer vs $M \Phi+$ apoptotic cells. B. $M \Phi+$ dimerizer vs $M \Phi+$ apoptotic cells. C. $M \Phi+$ apoptotic vs $M \Phi+$ necroptotic cells.

During MA plotting, fold change of genes against the mean of normalized counts of all samples were compared. A large number of genes showed significant changes in gene expression for each comparison of treatment groups (Figure 33). Differential expressed genes were selected based on a base mean $>0$ and a p-value of $<0.05$. Up- and downregulated genes were counted: Compared to control, 2410 genes were upregulated and 2359 genes downregulated in macrophages co-cultured with apoptotic cells. In 
macrophages co-cultured with necroptotic cells 2965 genes were upregulated and 3029 genes were downregulated compared to control. Compared to macrophages co-cultured with apoptotic cells, 2780 genes were upregulated and 2934 genes were downregulated in macrophages co-cultured with necroptotic cells.

A

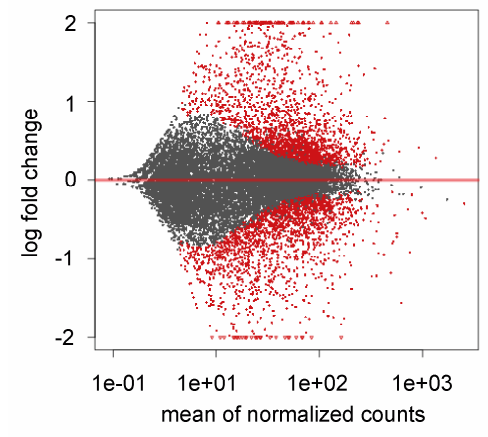

B

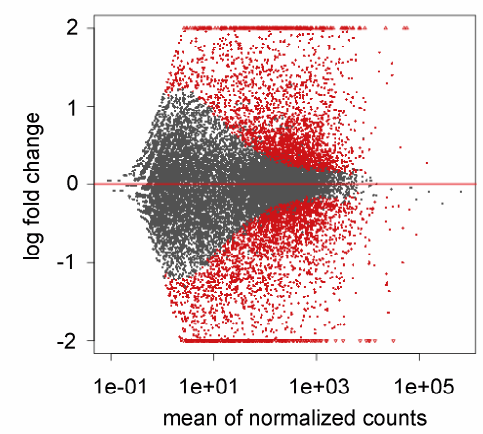

C

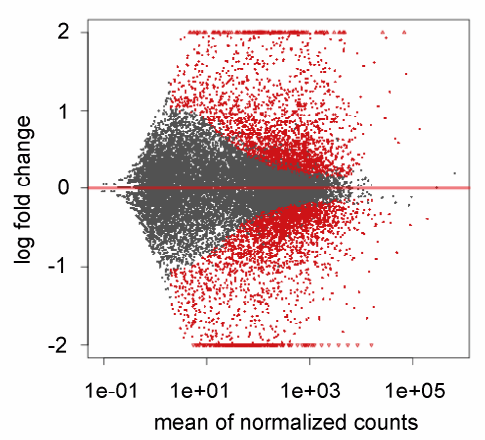

Figure 33. MA plots show the log fold change relative to mean expression. Differentially expressed genes are depicted in red. A. MФ+dimerizer vs $M \Phi+$ apoptotic cells. B. $M \Phi+$ dimerizer vs $M \Phi+$ apoptotic cells. C. MФ+apoptotic vs MФ+necroptotic cells.

Heat maps of normalized counts with a base mean of higher than 30 and p-value lower than 0.05 were created using Morpheus matrix visualization and analysis software. Subsequently, hierarchical clustering was performed (Figure 34). Reproducibility of biological replicates could be confirmed as replicates clustered together. Taken together, these analyses could verify that co-culture with dying cells led to significant changes in gene expression of peritoneal macrophages. 
A

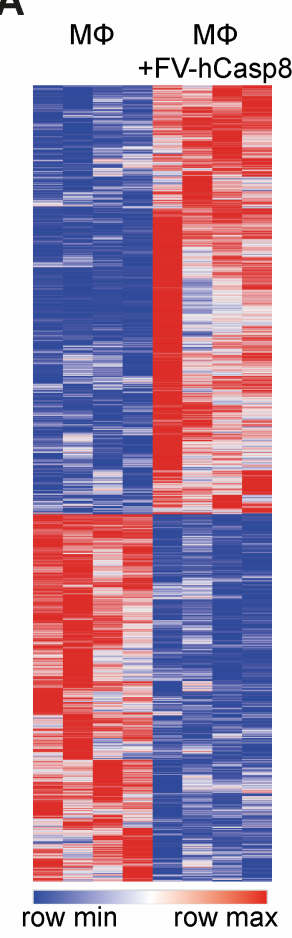

B
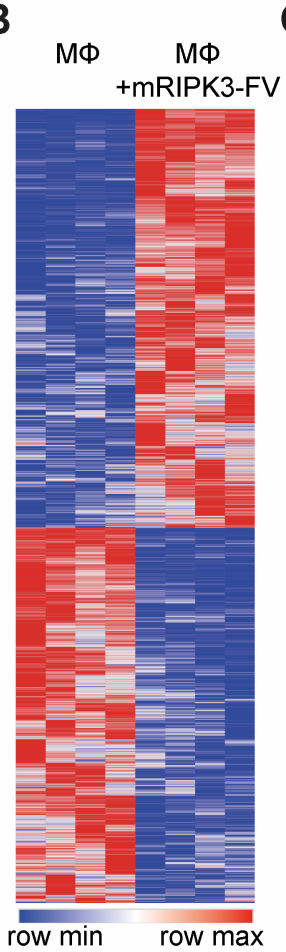

C
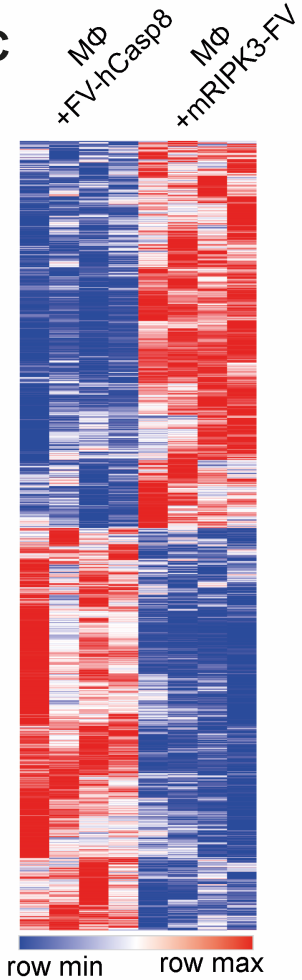

Figure 34. Heat maps and hierarchical clustering of normalized counts with base mean $>30$ and $p$ value $<\mathbf{0 . 0 5}$ was performed using Morpheus. A. Comparing $М \Phi$ and $M \Phi+$ apoptotic cells, 2905 genes were differentially expressed. B. Comparing $M \Phi$ and $M \Phi+$ necroptotic cells, 3905 genes were differentially expressed. C. Comparing $\mathrm{M} \Phi+$ apoptotic cells and $\mathrm{M} \Phi+$ necroptotic cells, 4822 genes were differentially expressed.

Next, we analyzed the signaling pathways leading to the alternated gene expression profiles in macrophages co-cultured with dying cells by performing gene set enrichment analysis (GSEA) and gene ontology analysis (GO). GSEA showed enriched gene sets of $\mathrm{PGE}_{2}$ signaling in macrophages co-cultured with apoptotic cells, both in comparison to control and macrophages co-cultured with necroptotic cells (Figure $35 \mathrm{~A}$ and $37 \mathrm{C}$ ). Interestingly, cell cycle and replication gene sets were enriched in these cells as well (Figure $35 \mathrm{~B}-\mathrm{D}$ and $37 \mathrm{~A}, \mathrm{~B}, \mathrm{D}$ ), indicating that macrophages are proliferating after contact with apoptotic cells. 

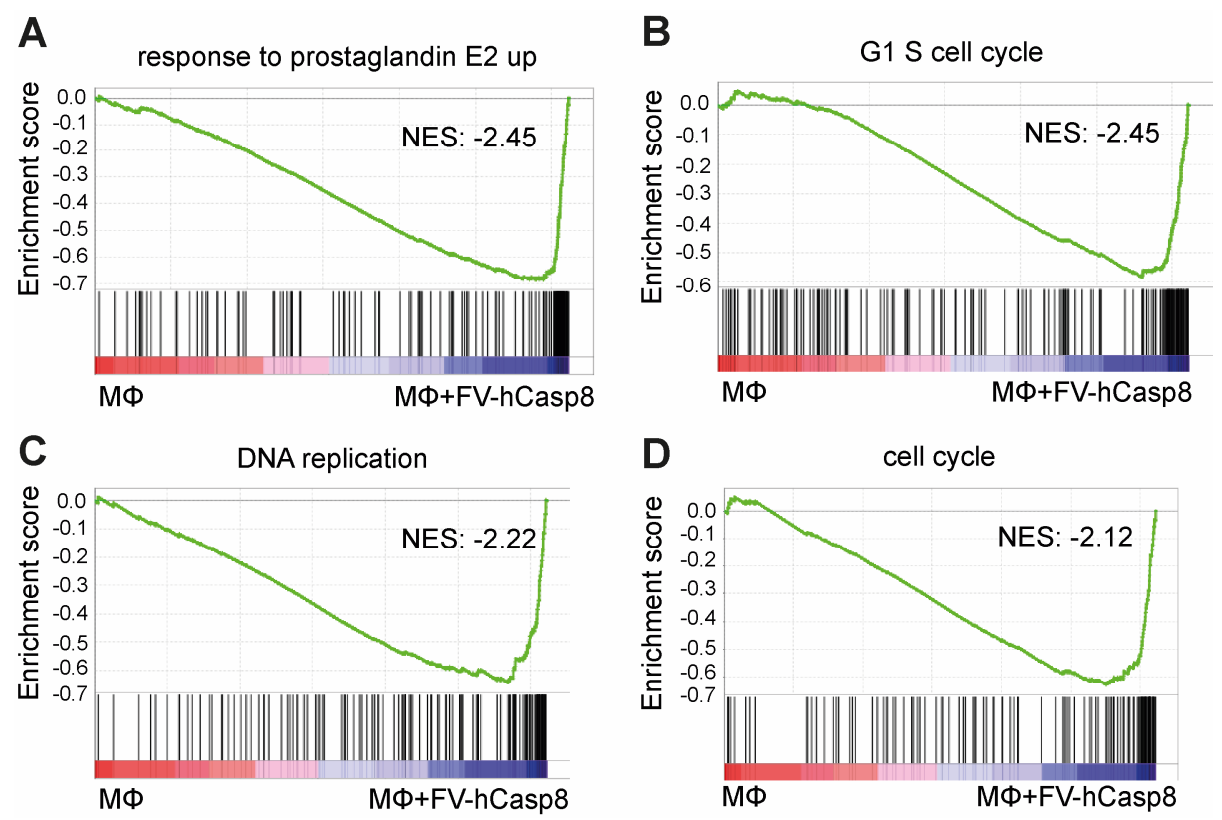

Figure 35. In macrophages co-cultured with apoptotic cells compared to control gene sets of cell cycle and replication were enriched after GSEA. Einrichment blots of respone of prostaglanding (A), G1 S cell cycle (B), DNA replication (C) and cell cycle (D) and corresponding normalized enrichment scores (NES) are shown.

Co-culture with necroptotic cells led to enrichment of hypoxia and HIF-1 $\alpha$ pathway gene sets compared to control and macrophages co-cultured with apoptotic cells (Figure 36 and $37 \mathrm{E}-\mathrm{H})$. This indicated that co-culture with necroptotic cells might influence the metabolism of macrophages as activation of the HIF-1 $\alpha$ pathway is activated in M1 macrophages, which show enhanced glycolysis and reduced levels of mitochondrial activity [38]. 

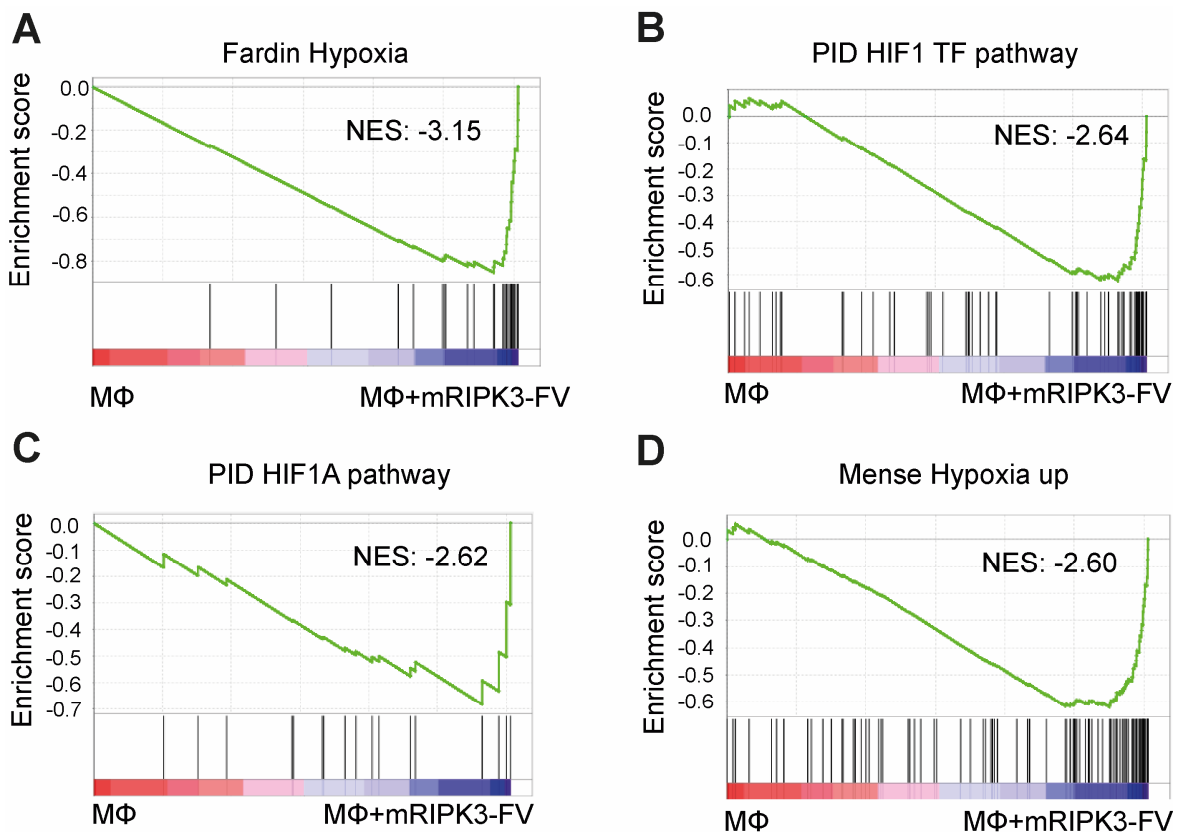

Figure 36. In macrophages co-cultured with necroptotic cells compared to control gene sets of hypoxia and the HIF1 pathway were enriched. Einrichment blots of respone of Fardin hypoxia (A), PID HIF1 TF pathway (B), PID HIF1A pathway (C) and Mense hypoxia (D) and corresponding normalized enrichment scores (NES) are shown. 
A

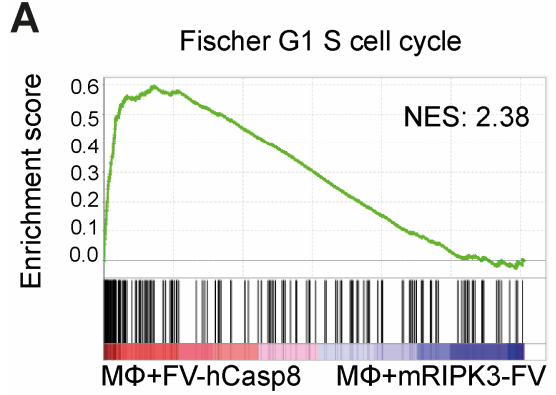

C Chemnitz response to prostaglandin E2

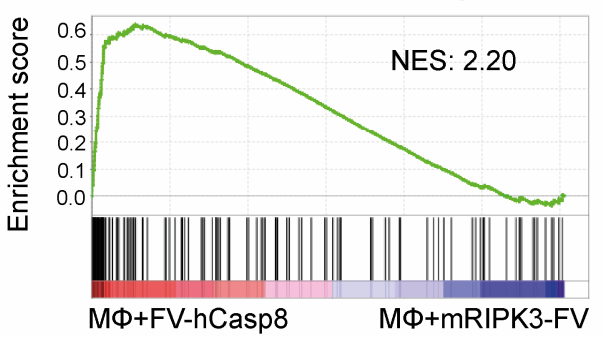

E

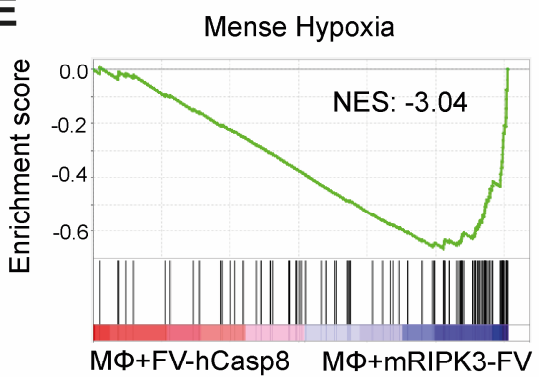

G

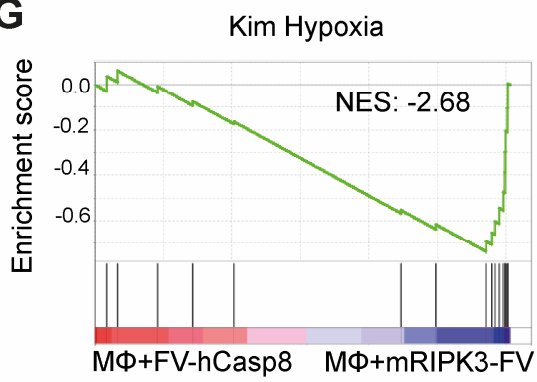

B

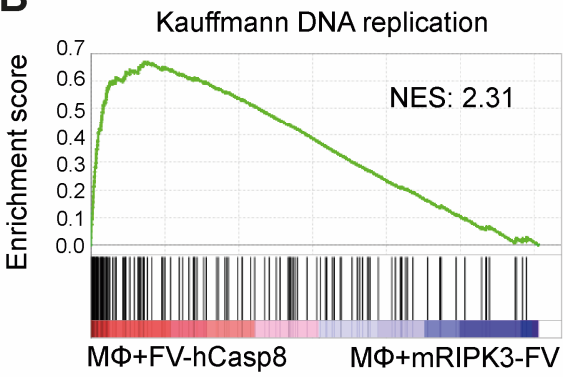

D

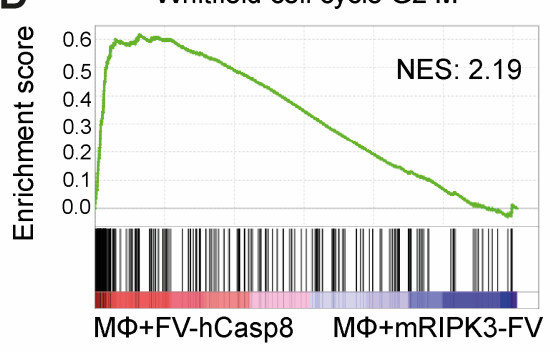

F PID HIF1 TF pathway

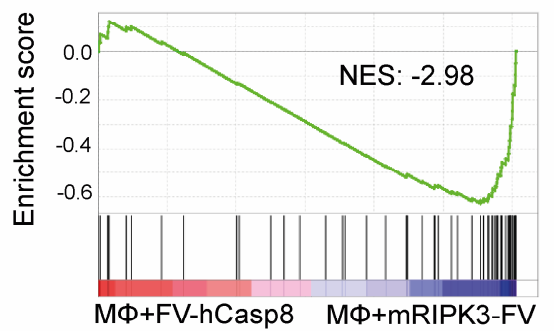

H

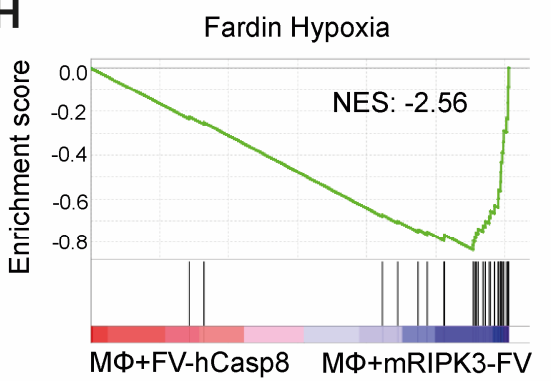

Figure 37. Gene sets enriched in macrophages co-cultured with necroptotic cells compared to macrophages co-cultured with apoptotic cells are shown. Einrichment blots of Fischer G1 S cell cycle (A), Kauffmann DNA replication (B), Chemnitz response to prostaglandin E2 (C), Whitfield cell cycle G2 M (D), Mense Hypoxia (E), PID HIF1 TF pathway (F), Kim hypoxia (G) and Fardin hypoxia (H) and corresponding normalized enrichment scores (NES) are depicted.

Consistent with GSEA analysis, GO analysis showed mainly upregulation of cell cycle and cell division pathways in macrophages co-cultured with apoptotic cells (Figure $38 \mathrm{~A}$ ). Interestingly, in macrophages co-cultured with necroptotic cells pathways indicating chemotaxis and leukocyte migration were upregulated (Figure $38 \mathrm{~B}$ and C). 
A

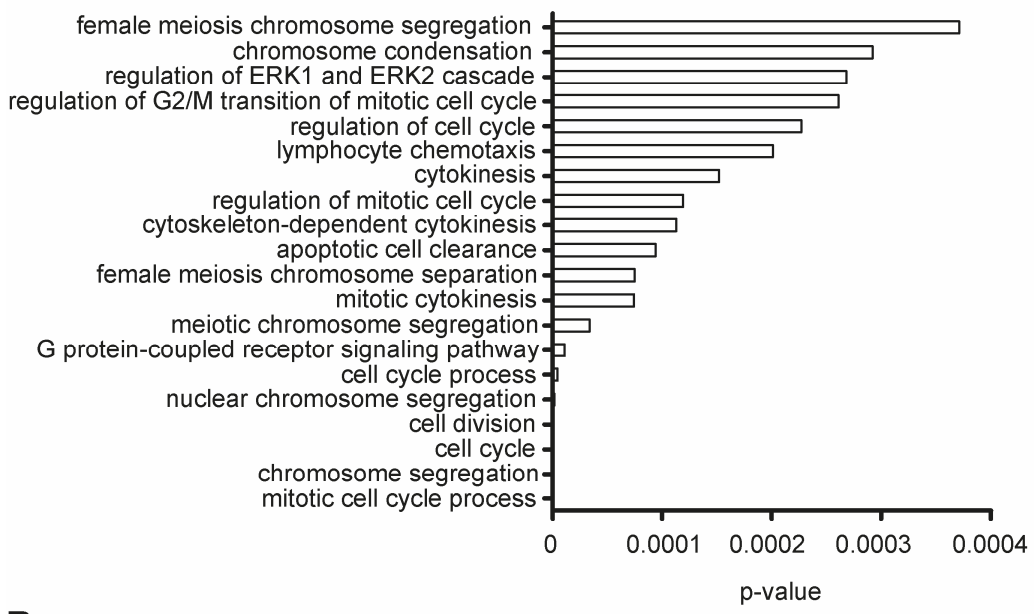

B

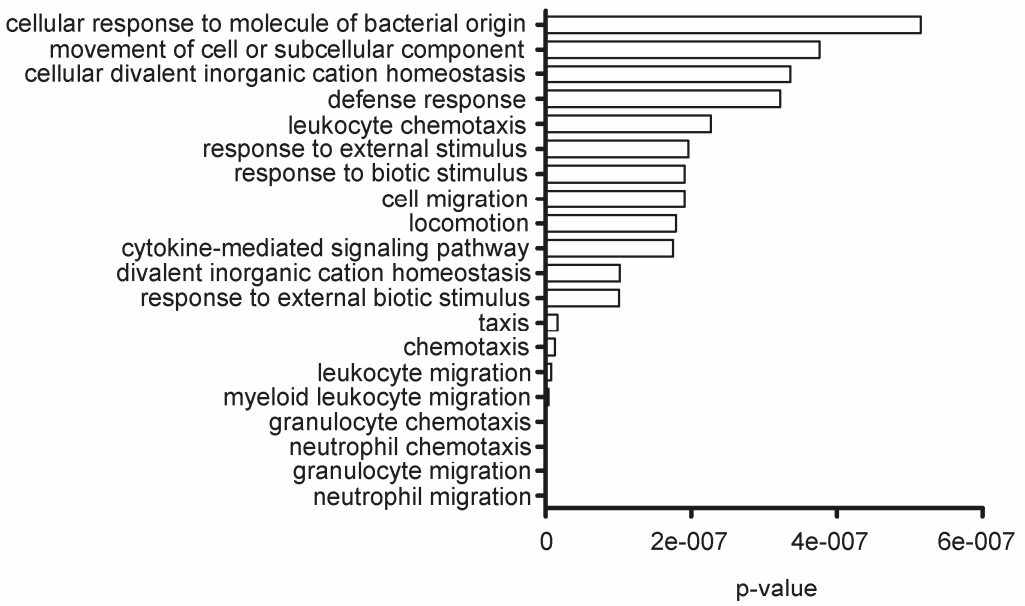

C

leukocyte chemotaxis cellular response to molecule of bacterial origin negative regulation of biological process negative regulation of biological process
negative regulation of mulitcellular organismal process
regulation of signal transduction
cellular response to lipopolysaccharide cellular response to lipopolysaccharide granulocyte chemotaxis neutrophil chemotaxis immune system process leukocyte migration cellular response to lipid regulation of response to stimulus granulocyte migration myeloid leukocyte migration neutrophil migration cytokine-mediated signaling pathway positive regulation of developmental process chemotaxis taxis -1

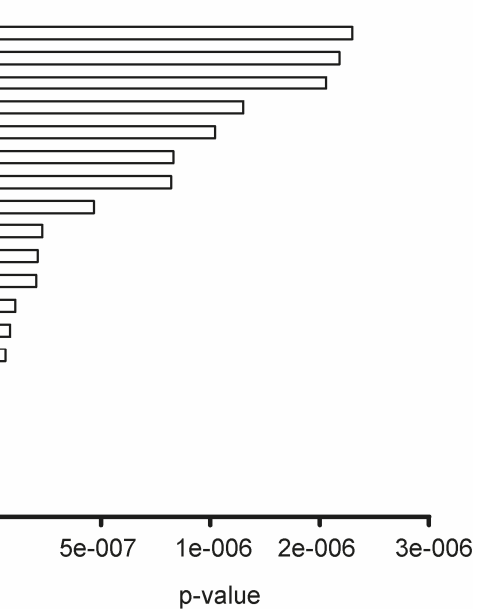

Figure 38. Gene ontology analysis using GOrilla confirmed results of the GSEA analysis. A. Top 20 upregulated pathways of macrophages co-cultured with apoptotic cells compared to control after GO analysis are shown. B. Top 20 upregulated pathways of macrophages co-cultured with necroptotic cells compared to control after GO analysis are shown. C. Top 20 upregulated pathways of macrophages cocultured with necroptotic cells compared to macrophages co-cultured with apoptotic cells after GO analysis are shown. 


\subsubsection{Apoptotic cells induce proliferation of macrophages}

RNA sequencing revealed upregulation of cell cycle pathways in macrophages cocultured with apoptotic cells. Therefore, we validated these finding by analyzing mRNA level of cell cycle genes and the proliferation of macrophages during co-culture with apoptotic cells. Expression of MKi67, Top2a, Aurkb and Cdk1 at mRNA level were significantly upregulated in macrophages co-cultured with apoptotic cells compared to macrophages co-cultured with dimerizer alone (Figure 39 A).

Next, we wanted to show if these macrophages are proliferating. Macrophages were cocultured alone or with FV-hCasp8 cells and cell death of FV-hCasp8 cells was induced by dimerizer treatment. Confluence was measured every 4 hours for 48 hours as indicator for proliferation (Figure 39 B and Figure 40). Co-culture with NIH 3 T3 cells seemed to have a beneficial effect for macrophages as macrophages co-cultured alone started to die shortly after beginning of the measurement, whereas confluence in wells with macrophages co-cultured with living FV-hCasp8 increased slightly. Confluence in wells with macrophages co-cultured with apoptotic cells significantly inceased after 48 hours compared to the co-culture with living NIH $3 \mathrm{~T} 3$ cells. This findings indicated that coculture of macrophages with apoptotic cells resulted in proliferation of macrophages.
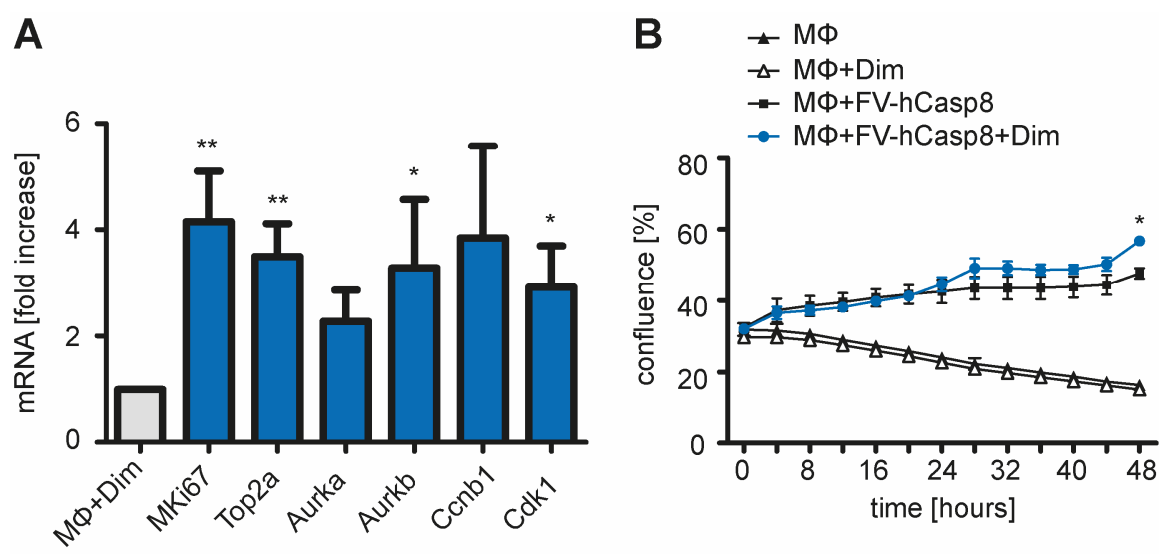

Figure 39. Co-culture with apoptotic cells leads to proliferation of primary peritoneal macrophages. A. Macrophages were co-cultured with apoptotic cells and mRNA of macrophages was isolated. Fold change of mRNA is shown as mean + SEM. $n=4$. B. Macrophages were co-cultured with FV-hCasp8 cells and apoptosis was induced by dimerizer treatment. Confluence was measured every 4 hours for 48 hours. Experiments were performed with the help of Zumer Naeem. Data are shown as mean \pm SEM. $n=3$. 
MФ
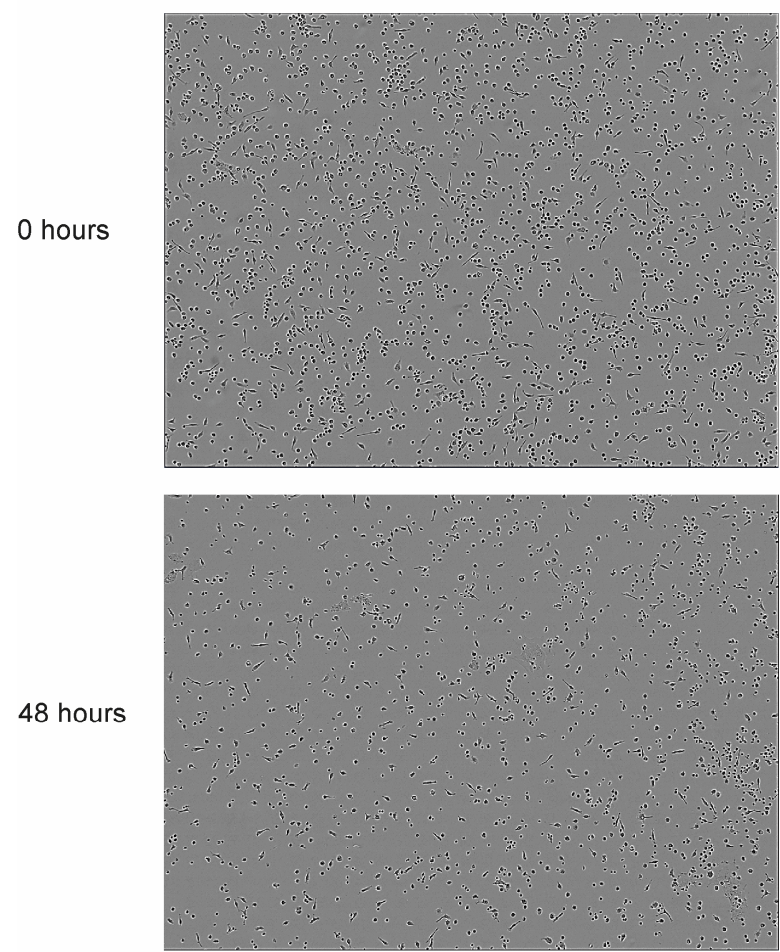

MФ+FV-hCasp8

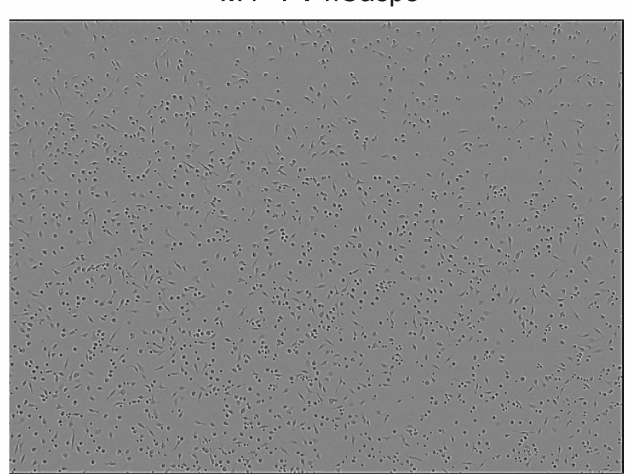

0 hours

48 hours

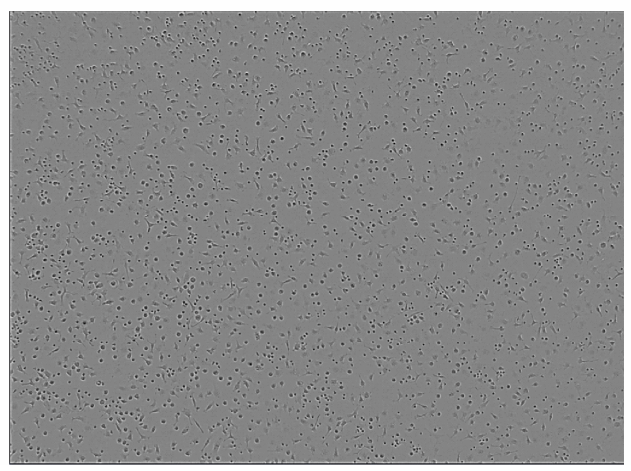

МФ+dimerizer
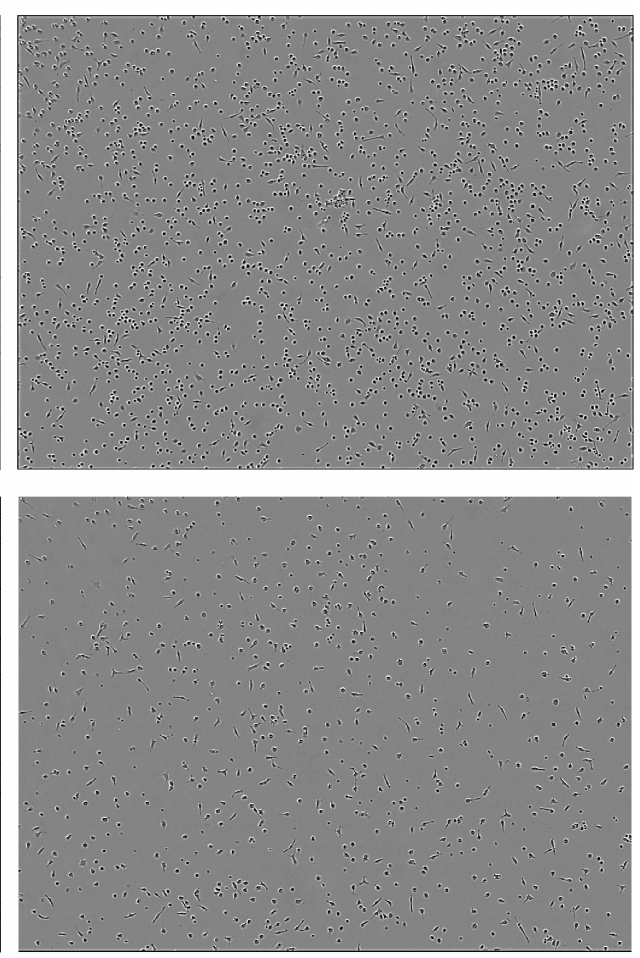

MФ+FV-hCasp8+dimerizer
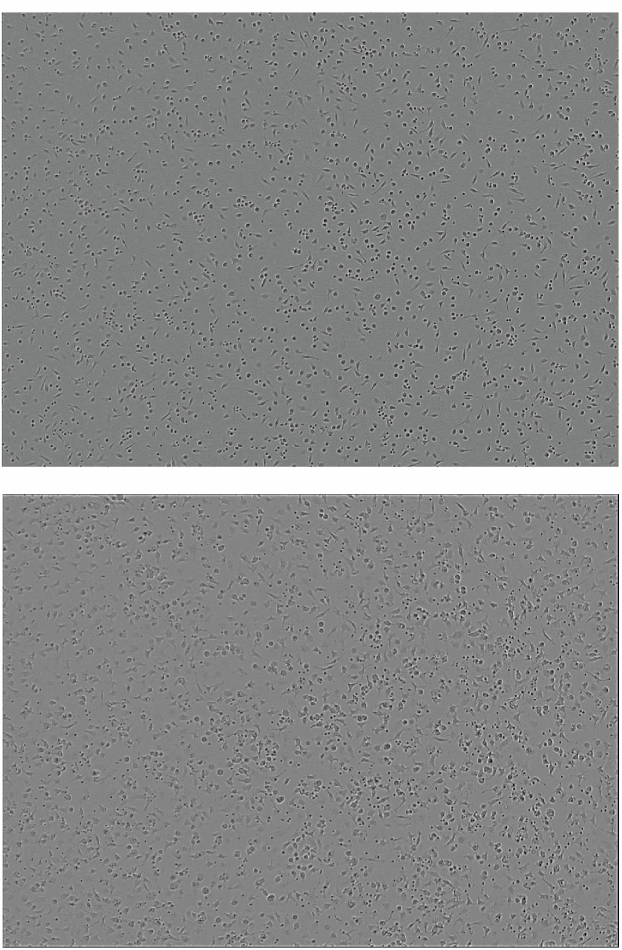

Figure 40. Images of macrophages before and after co-cultivation with apoptotic cells. Macrophages were co-cultured with FV-hCasp8 cells and apoptosis was induced by dimerizer treatment. Images were taken during measurement of confluence for 48 hours. Experiments were performed with the help of Zumer Naeem. One representative well per condition is shown. 
To confirm this finding in another system, we used the IC-21 peritoneal macrophage cell line. IC-21 cells were stained with eFluor 670 and co-cultured with FV-hCasp8 or mRIPK3-FV cells. Cell death was induced with dimerizer and mean fluorescence intensity of eFluor 670 was analyzed (Figure 41). eFluor 670 is a fluorescent dye, which binds to any protein in the cell containing primary amines and is diluted during proliferation of cells. The histogram in Figure 41 A shows in grey IC-21 control cells, which are stained with eFluor 670 and do not proliferate. Most of the cells have a high fluorescence intensity. IC-21 cells co-cultured with apoptotic cells (in blue) showed a shift of fluorescence intesity to the left, indicating that these cells proliferated. Indeed, analyzing the mean fluorencence intensity of the cells after co-culture showed a significant decrease in cells co-cultured with apoptotic cells (Figure $41 \mathrm{~B}$ ). Co-culture with necroptotic cells did not significantly change fluorescence intensitiy and did not lead to proliferation of IC-21 cells.
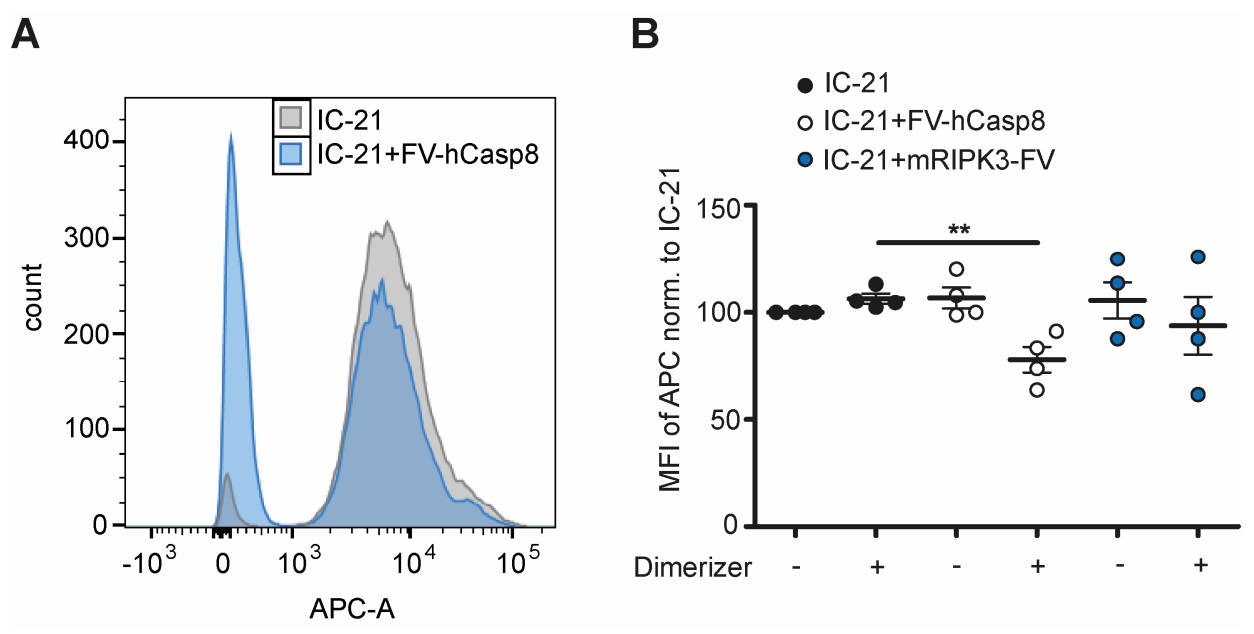

Figure 41. Co-culture with apoptotic cells leads to proliferation of IC-21 cells. A. IC-21 cells were stained with eFluor 670 and co-cultured with apoptotic cells for 24 hours. The histogram shows fluorescence intensity of IC-21 control cells or IC-21 cells co-cultured with apoptotic cells. B. Mean fluorescence intensity (MFI) of IC-21 cells stained with eFluor 670 after co-culture was measured using flow cytometry. Data are shown as mean \pm SEM. $n=4$.

\subsubsection{Necroptotic cells affect chemokine profil in macrophages}

During RNA sequencing of macrophages co-cultured with necroptotic cells, hypoxia and chemotaxis pathways were upregulated. Activation of the hypoxia pathway indicates changes in the metabolism of macrophages. Metabolic changes are part of macrophage polarization. M1 macrophages tend to be more glycolytic active and M2 macrophages depend more on mitochondrial respiration. 
Therefore, we analyzed changes in the energy metabolism of macrophages co-cultured with necroptotic cells using a Seahorse assay (Figure 42). Here, in an automated measurement cellular oxygen consumption (oxygen consumption rate, OCR) as an indicator for respiration and proton secretion (extracellular acidification rate, ECAR) as an indicator for glycolysis can be measured in real time [60]. To determine the OCR and test key parameters of mitochondrial respiration, a Cell Mito Stress test was performed. During this test, different modulators of respiration are added and OCR is measured (Figure 42). Oligomycin, which inhibits the ATP synthase, is added first and the reduction in mitochondrial respiration linked to cellular ATP production can be measured. Next, the uncoupling agent FCCP is added. FCCP disruptes the mitochondiral membrane potential and the oxygen consumption by complex 4 will reach its maximum, which allows to calculate the spare respiratory capacity. In the end, Rotenone, which is a complex I inhibitor and Antimycin A, which is a complex III inhibitor are added completely shutting down mitochondrial respiration to calculate nonmitochondrial respiration.

A

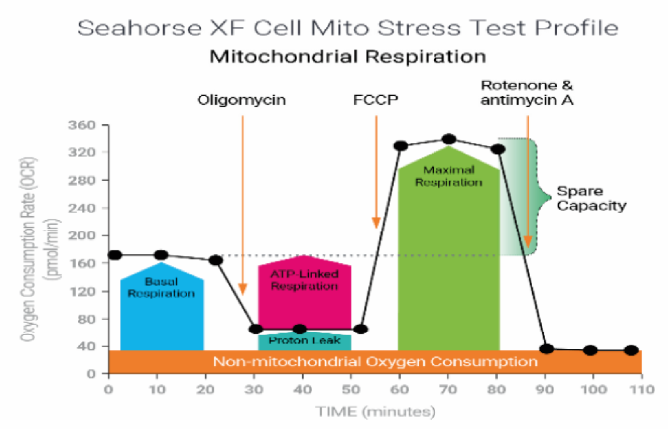

B

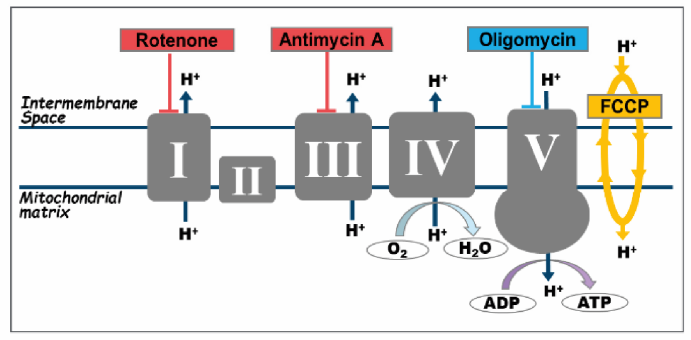

Figure 42. The oxygen consumption rate can be measured by a Cell Mito Stress test. A. The timeline of the Seahorse XF Cell Mito Stress test is depicted. B. Different respiratory modulators are added during the stress test, which inhibit different parts of the electron transport chain. A.+B. Modified after Agilent Seahorse XF Cell Mito Stress Test Kit User Guide.

The maximal mitochondrial respiration (calculated by addition of FCCP) was significantly reduced in macrophages co-cultured with necroptotic cells compared to macrophages co-cultured with living fibroblasts, whereas macrophages co-cultured with living cells showed a significantly higher maximal mitochondrial respiration compared to control cells (Figure $43 \mathrm{~A}$ ). This indicated that macrophages are polarized towards M1 phenotype, when co-cultured with necroptotic cells. The extracellular acidification rate was not changed during co-culture, indicating that glycolysis rates were not changed 
(Figure 43 B). mRNA expression levels of HIF-1 $\alpha$ target genes were not changed (data not shown).
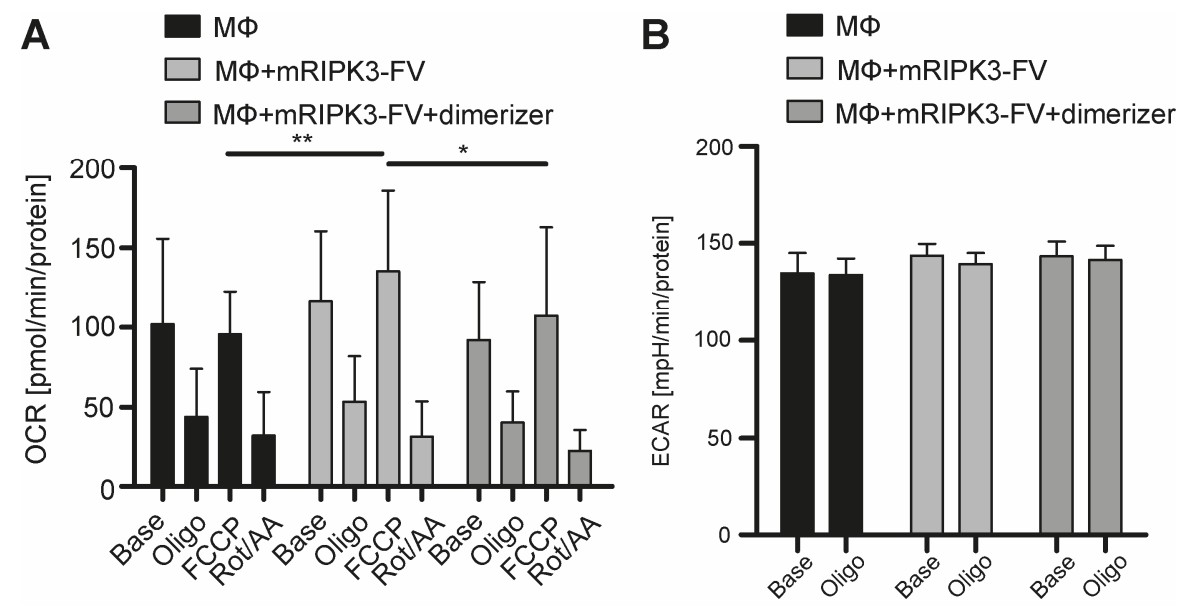

Figure 43. Maximal mitochondrial respiration in macrophages co-cultured with necroptotic cells is significantly reduced. A. Macrophages were co-cultured with living or necroptotic cells and a Cell Mito Stress test was performed to anaylze the oxygen consumption rate (OCR). Data are shown as mean + SEM. $\mathrm{n}=3$. B. Macrophages were co-cultured with necroptotic cells and extracellular acidification rate (ECAR) was analyzed. Experiments were performed with the help of Dominik Fuhrmann. Data are shown as mean + SEM. $\mathrm{n}=3$.

Chemotaxis pathways were also upregulated during RNA sequencing in macrophages cocultured with necroptotic cells. Therefore, we analyzed chemokine levels in the supernatant after co-culture of macrophages with necroptotic cells. Among 13 different measured chemokines, CXCL1 and CXCL13 protein levels were regulated significantly (Figure 44). Levels of the neutrophil chemotractant CXCL1 were significant upregulated in the supernatant of the co-culutre of macrophages and necroptotic cells (Figure 44 A), which matches the RNA squencing data. However, necroptotic cells alone are secreting high levels of CXCL1, indicating, that CXCL1 in the supernatant of the co-culture is secreted by necroptotic cells.

Levels of CXCL13, which is able to attract B cells, were upregulated in the co-culture of macrophages with living cells and significantly reduced in the co-culture with necroptotic cells (Figure 44 B). In the RNA sequencing, CXCL13 was downregulated in macrophages co-cultured with necroptotic cells compared to control and compared to macrophages cocultured with apoptotic cells. Taken together, these experiments show, that necroptotic cells change the chemokine profile of macrophages. 

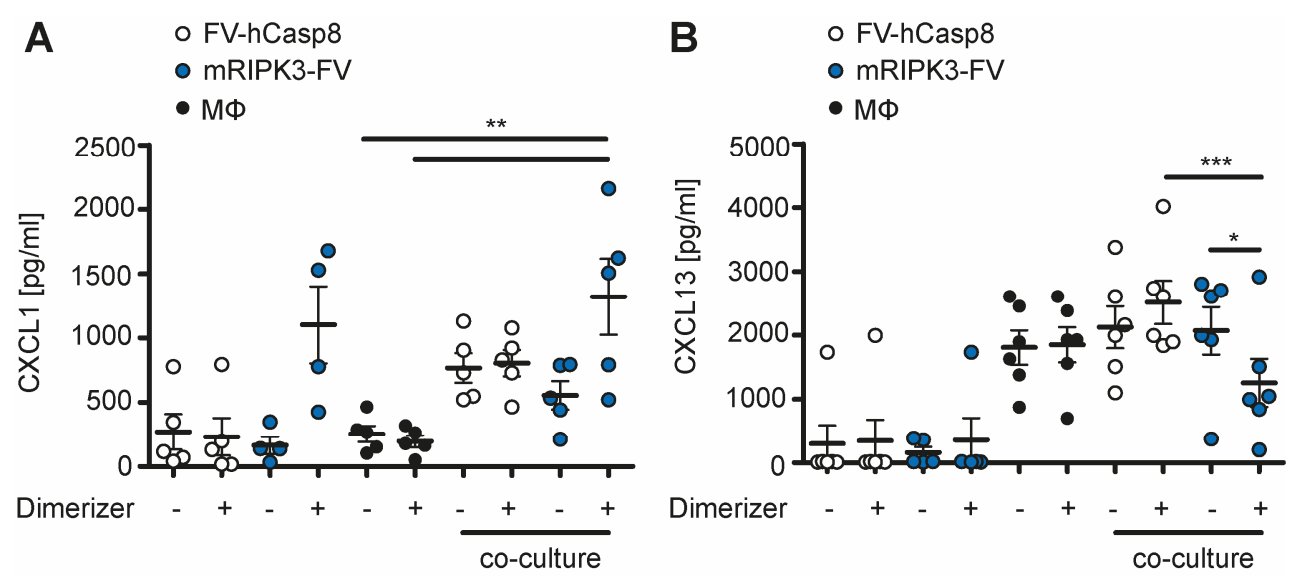

Figure 44. CXCL1 and CXCL13 levels were significantly regualted in macrophages co-cultured with necroptotic cells. NIH $3 \mathrm{~T} 3$ cells or peritoneal macrophages (MФ) were either cultured alone or together and cell death of NIH 3T3 cells was induced by dimerizer treatment. 48 hours after co-culture, protein levels of CXCL1 (A) and CXCL13 (B) were measured. Data are shown as mean \pm SEM. n=5-6.

\subsubsection{Effect of dying cells on resolution of inflammation in vivo}

Next, we wanted to further validate our findings in an in vivo model of inflammation and its resolution. We chose the model of Zymosan-A-induced peritonits in mice. Here, injection of $10 \mathrm{mg} / \mathrm{kg}$ Zymosan-A into the peritoneum of C57-BL/6 mice leads to a selfresolving acute inflammation. Zymosan-A is a yeast component, which is recognized by dectin-1 and toll-like receptor-2 (TLR2) on phagocytes. Phagocytosis of Zymosan-A leads to activation of nuclear factor $\kappa-\mathrm{B}$ and release of pro-inflammatory cytokines and chemokines [61].

During Zymosan-A-induced peritontis polymorphonuclear leukocytes like neutrophils infiltrate the tissue. Influx of neutrophils peaks around 4 hours and the inflammational response peaks at day 1 (Figure 45). With recruitment of macrophages and phagocytosis of neutrophils, resolution of inflammation starts at day 3. In the end, pro-inflammatory macrophages become anti-inflammatory and the inflammation is resolved after 6 days [62]. 


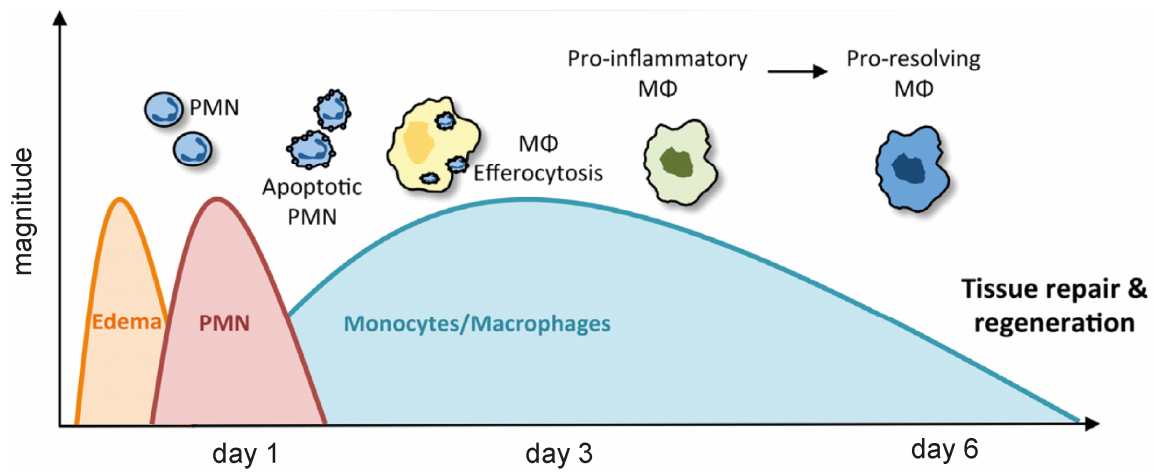

Figure 45. The time line of Zymosan-A-induced peritonitis is depicted. Influx of neutrophils peaks at day 1. Macrophages inflitrate the tissue and with this resolution starts at day 3. After 6 days resolution is complete. Figure modified after [36].

To analyze the role of apoptosis and necroptosis in resolution of inflammation, ZymosanA was injected into the peritoneum of C57-BL/6 mice and after 8 hours, right before the peak of inflammation, either dying cells or cell death inhibitors were injected (Figure 46). In the control group (Figure $46 \mathrm{~A}$ ) PBS with 1\% DMSO were injected. Apoptotic or necroptotic cells were produced in vitro using FV-hCasp8 or mRIPK3-FV dimerizer systems described above (Figure $46 \mathrm{~B}$ ). Inhibition of apoptosis or necroptosis was achieved by injection of the pan-caspase inhibitor zVAD.fmk or the RIPK3 inhibitor GSK'872, respectively (Figure 46 C). Cells, cytokines and chemokines were isolated by lavage of the peritoneum and analyzed 1, 3 and 6 days after Zymosan-A injection. 


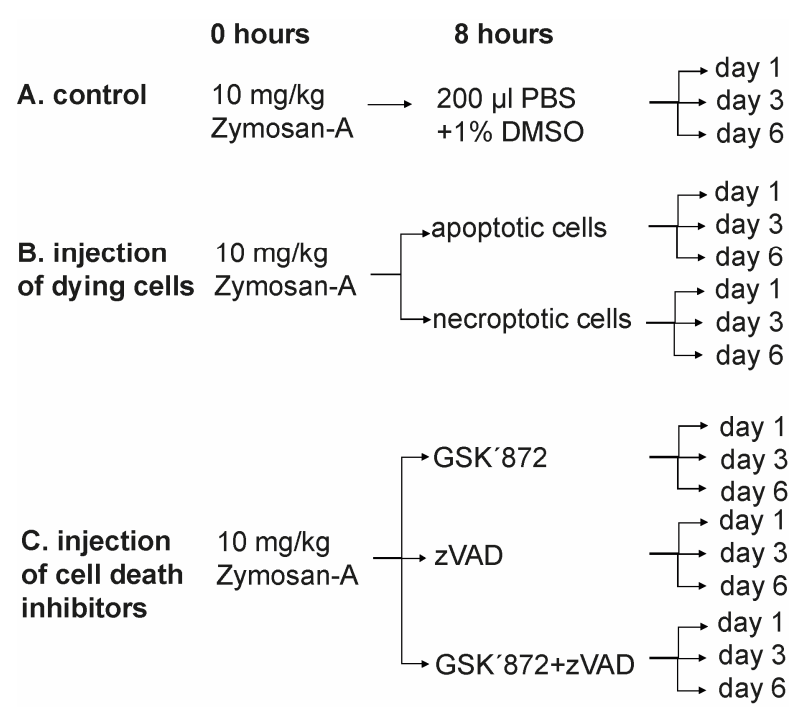

Figure 46. Treatment scheme of in vivo experiments is shown. A. Control animals were treated with $10 \mathrm{mg} / \mathrm{kg}$ Zymosan-A and after 8 hours PBS and 1\% DMSO were injected i.p. B. Apoptotic or necroptotic cells were injected 8 hours after Zymosan-A injection. C. Cell death was inhibited by GSK' 872 and/or zVAD.fmk injection 8 hours after Zymosan-A injection. Cells, cytokines and chemokines were analyzed at day 1,3 and 6.

Before dying cells were injected into mice, cell death was confirmed by Annexin-PI staining (Figure 47). FV-hCasp8 cells were treated with dimerizer for 8 hours when they started to become Annexin positive, indicating that cells show PS on their surface and are dying via apoptosis. mRIPK3 cells were treated for 4 hours with dimerizer and $80 \%$ of the cells are dying at this time point as they are PI positive.

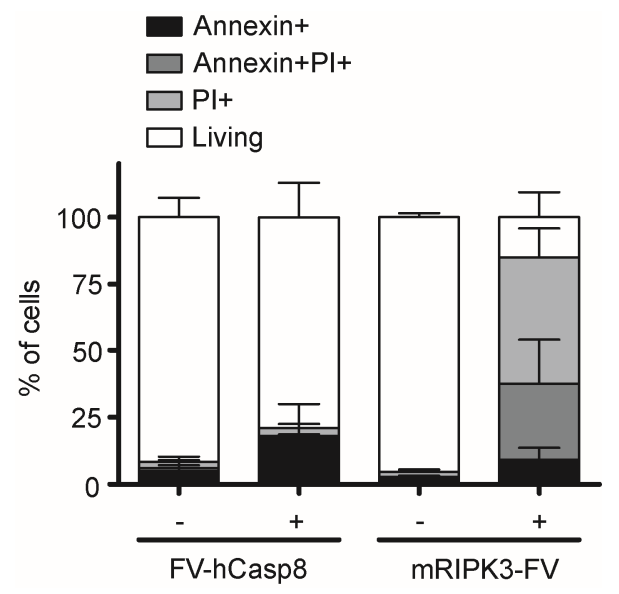

Figure 47. Cell death of FV-hCasp8 and mRIPK3-FV cells was confirmed before injection into mice. Cells were treated for 4 (mRIPK3-FV) or 8 hours (FV-hCasp8) with dimerizer and cell death was confirmed by Annexin-PI staining. Data are shown as mean + SEM. $n=3$. 
During Zymosan-A-induced peritonitis, weight of the mice was monitored daily (Figure 48). Successful induction of inflammation led to a weight loss of around $5 \%$ of the body weight (DMSO and Ctrl). On the one hand, injection of GSK'872 increased the weight at day 2 and 3 significantly (day 2:**, day $3:^{*}$ compared to DMSO), indicating that inhibition of necroptosis induced a faster resolution of inflammation (Figure $48 \mathrm{~A}$ ). On the other hand, injection of necroptotic cells significantly increased the weight at day 3 compared to control animals (**, Figure 48 B).
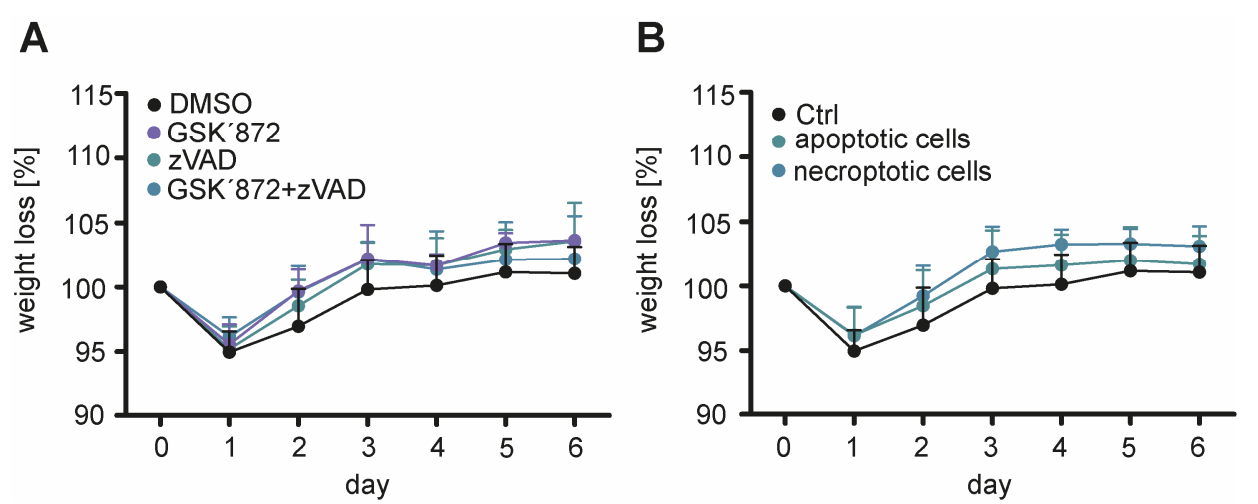

Figure 48. Weight loss of mice was monitored daily. A. Cell death inhibitors were injected 8 hours after Zymosan-A injection and mice were weight every day. $n=4-19$. B. Dying cells were injected 8 hours after Zymosan-A injection and mice were weight every day. $n=4-19$. Data are shown as mean + SEM.

Cells of the peritoneum were harvested at day 1, 3 and 6 and dead cells and immune cell profiles were analyzed. Injection of cell death inhibitors decreased the percentage of Annexin and PI positive cells at day 1 and 3, indicating, that the inhibtion of cell death was successful (Figure 49 A). Injection of dying cells did not alter the percentage of dead cells in the peritoneal lavage (Figure 49 B). 
A

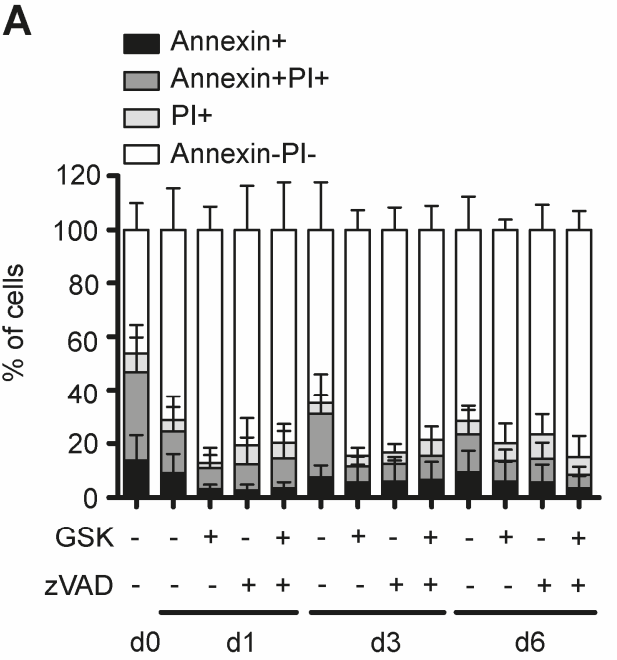

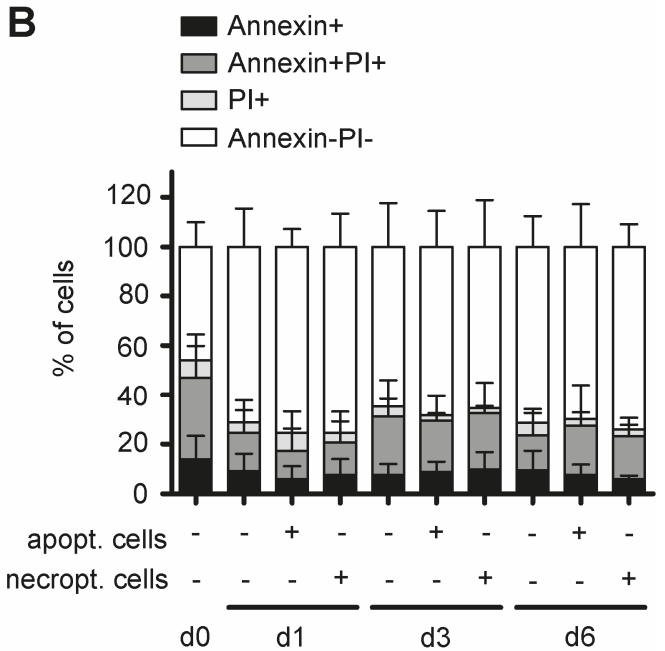

Figure 49. Death of cells in the lavage was analyzed at day 1, 3 and 6 via Annexin PI staining. A. Cell death inhibitors were injected 8 hours after Zymosan-A injection and dead cells in the lavage were analyzed. $\mathrm{n}=5-10$. B. Dying cells were injected 8 hours after $-\mathrm{A}$ injection and and dead cells in the lavage were analyzed. $n=6-10$. Data are shown as mean \pm SEM.

Next, the immune cell profile of the lavage was analyzed by staining cells with antibodies against different immune cell markers using flow cytometry. The gating strategy to identify different immune cells in the peritoneum is shown in the methods (see section 3.2.6.4). To better visualize these high-dimensional data, $\mathrm{t}$-distributed stochastic neighbor embedding (t-SNE) analysis was performed. In this statistical method cells with similar protein expressions are located close together in the scatter plot, allowing to observe cell clusters and their changes during treatment [63]. Figure 50 shows t-SNE maps of control animals. Zymosan-A was injected and cells of the lavage were analyzed at day 1, 3 and 6. Before induction of inflammation (at day 0 ) the peritoneum consisted mostly of resident macrophages and B cells (Figure 50 A). One day after Zymosan-A injection, at the peak of inflammation, neutrophils infiltrated the peritoneum and monocyte-derived macrophages were recruited (Figure $50 \mathrm{~B}$ ). At day 3, when resolution of inflammation starts, the number of neutrophils was decreased, clusters of resident macrophages and monocyte-derived macrophages increased and a distinct cluster of NK cells had formed (Figure $50 \mathrm{C}$ ). At day 6 the peritoneum consisted again mostly of macrophages, monocytes and B cells. We could also observe a cluster of CD8+ memory T-cells (Figure $50 \mathrm{D})$. 
A

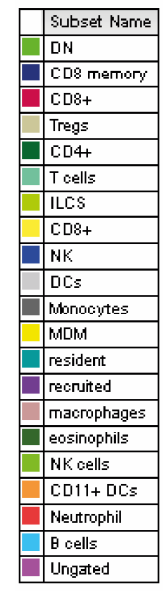

C
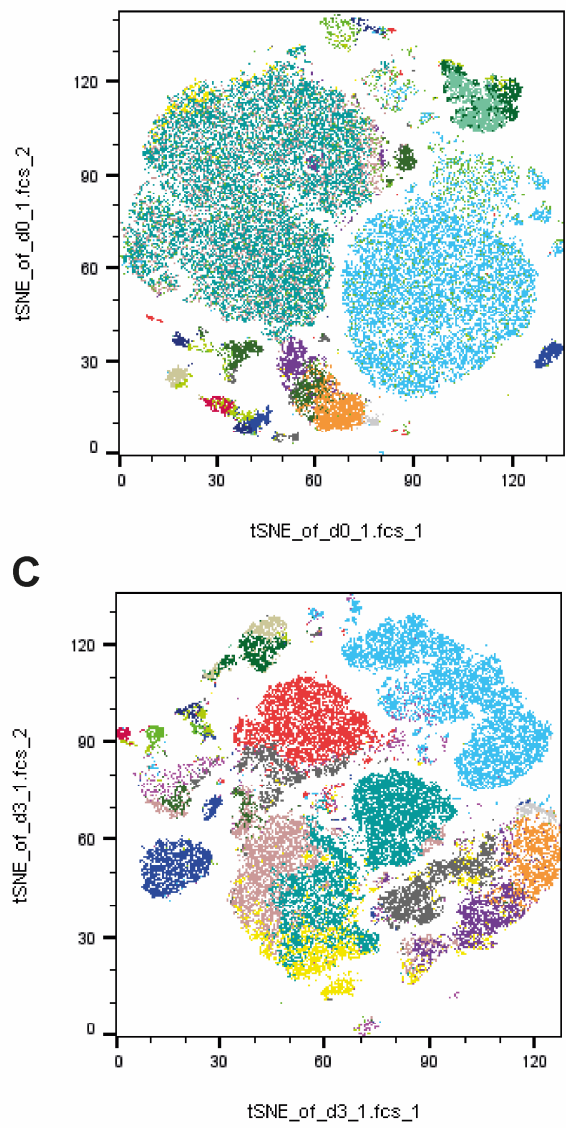

B

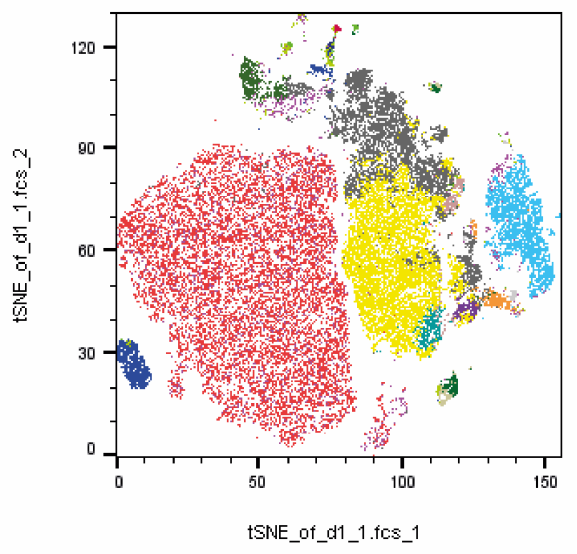

D

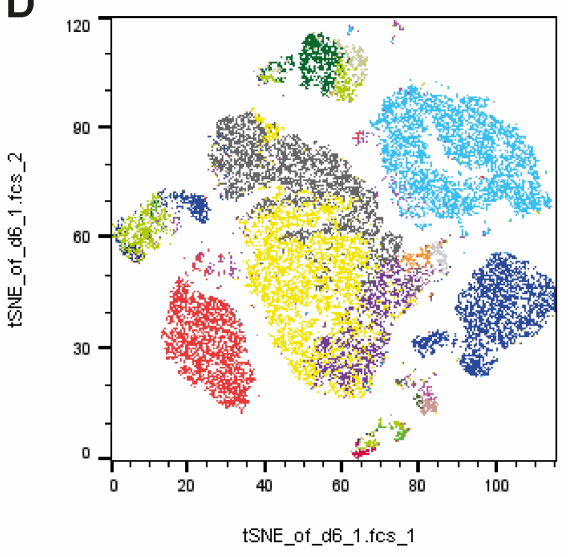

Figure 50. t-SNE maps of control animals show immune cell profiles during Zymosan-A-induced peritonitis. Cells of the peritoneum at day 0 (A) and 1 (B), 3 (C) and 6 days (D) after Zymosan-A-injection were isolated and analyzed by staining with different immune cell markers and flow cytometry. After gating, $\mathrm{t}$-SNE analysis was performed. $\mathrm{n}=3-7$.

Injection of cell death inhibitors 8 hours after Zymosan-A injection led only to small changes in the composition of immune cells (Figures 51 to 53). At day 1 the cluster of neutrophils was decreased in animals treated with GSK'872 (Figure 51 A) compared to animals treated with zVAD.fmk or both (Figure 51 B and C). Slightly more B cells were present in animals treated with zVAD.fmk at day 3 (Figure 52 B). At day 6 no big differences were observed in mice treated with cell death inhibitors (Figure 53). 
A

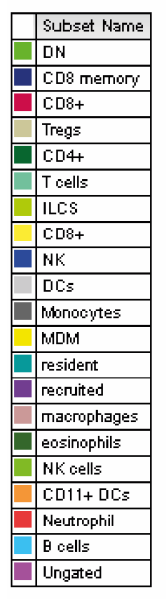

C
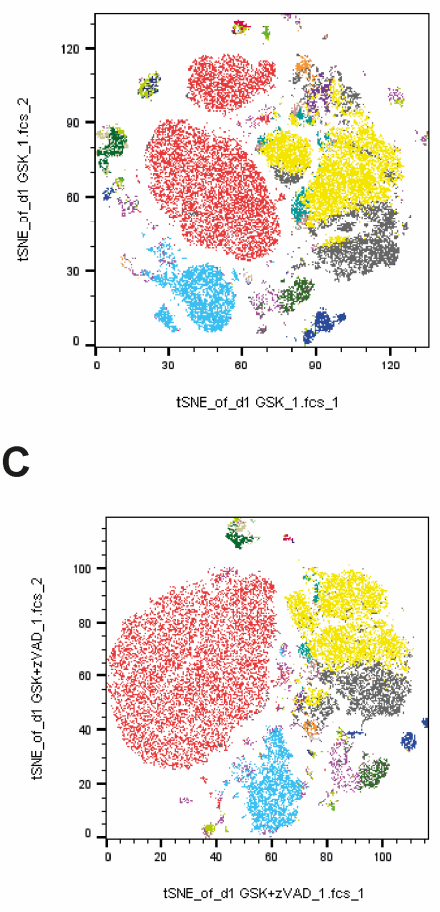

B

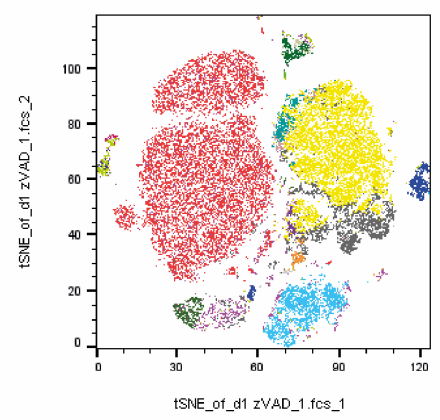

Figure 51. t-SNE maps of animals treated with cell death inhibitors show immune cell profiles during Zymosan-A-induced peritonitis at day 1. Cells of the peritoneum after Zymosan-A and GSK'872 (A), zVAD.fmk (B) injection or injection of both inhibitors (C) were isolated at day 1 and analyzed by staining with different immune cell markers and flow cytometry. After gating, t-SNE analysis was performed. $n=3$ 7. 
A

\begin{tabular}{|c|c|}
\hline & Subset Name \\
\hline & DN \\
\hline & CD8 memory \\
\hline & $\mathrm{CD} 8+$ \\
\hline & Tregs \\
\hline & $\mathrm{CD} 4+$ \\
\hline & Teells \\
\hline & ILCS \\
\hline & $\mathrm{CD} 8+$ \\
\hline & NKK \\
\hline & $\overline{D C s}$ \\
\hline & Monocytes \\
\hline & $M D M$ \\
\hline & resident \\
\hline & recrited \\
\hline & macrophages \\
\hline & eosinophils \\
\hline & NK cells \\
\hline & $\mathrm{CD} 11+\mathrm{DCs}$ \\
\hline & Neutrophil \\
\hline & B cells \\
\hline & Unigated \\
\hline
\end{tabular}

C

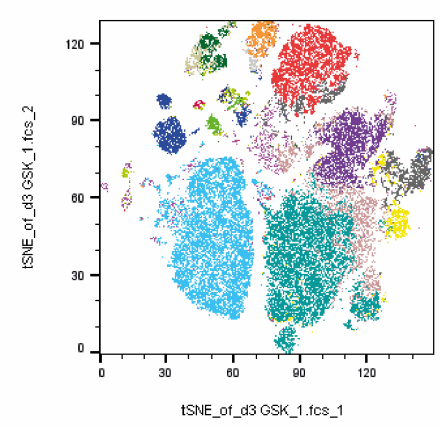

B

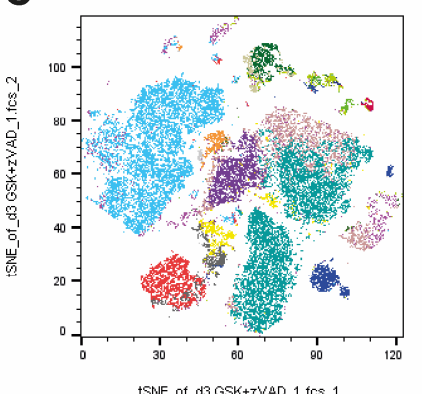

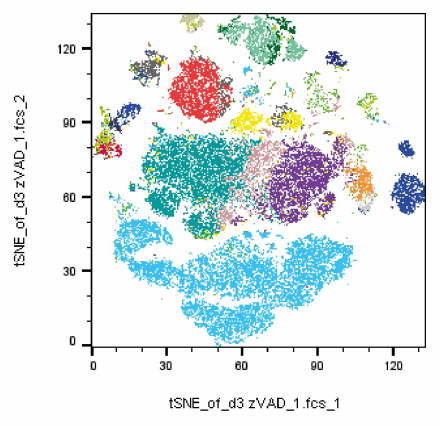

Figure 52. t-SNE maps of animals treated with cell death inhibitors show immune cell profiles during Zymosan-A-induced peritonitis at day 3. Cells of the peritoneum after Zymosan-A and GSK'872 (A), zVAD.fmk (B) injection or injection of both inhibitors (C) were isolated at day 3 and analyzed by staining with different immune cell markers and flow cytometry. After gating, t-SNE analysis was performed. $n=3-$ 7. 


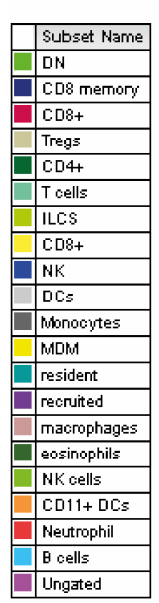

A

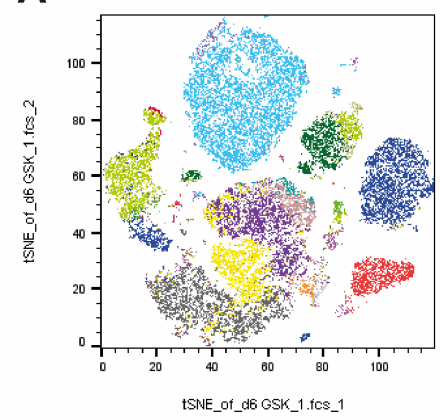

C

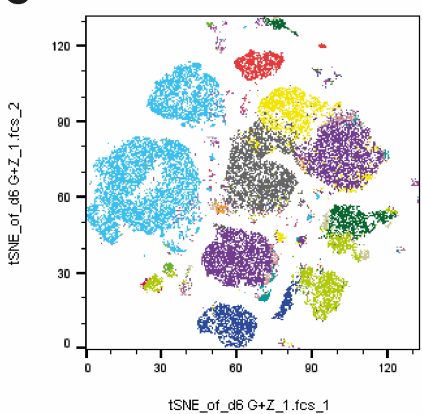

B

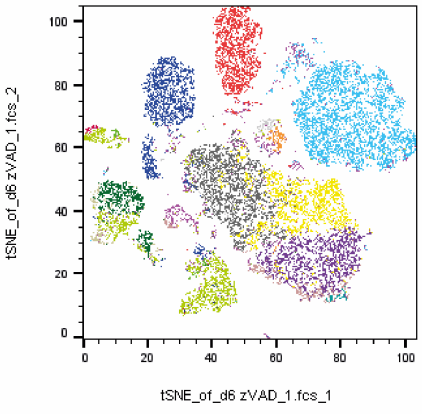

Figure 53. t-SNE maps of animals treated with cell death inhibitors show immune cell profiles during Zymosan-A-induced peritonitis at day 6. Cells of the peritoneum after Zymosan-A and GSK'872 (A), zVAD.fmk (B) injection or injection of both inhibitors (C) were isolated at day 6 and analyzed by staining with different immune cell markers and flow cytometry. After gating, t-SNE analysis was performed. $\mathrm{n}=3$ 7.

Immune cells were analyzed as percentage of cells or cell numbers were counted using an internal counting standard (beads). Inhibition of necroptosis during Zymosan-Ainduced peritonitis resulted in signficantly decreased percentages and numbers of neutrophils in the peritoneum at day 1 (Figure $54 \mathrm{~A}$ and B), indicating that reduced levels of necroptosis during inflammation led to reduced recruitment of neutrophils. Injection of zVAD.fmk and thereby inhibition of apoptosis increased percentages of B cells at day 3 (Figure $54 \mathrm{C}$ and D). Levels of macrophages were not significantly changed by injection of cell death inhibitors (Figure $54 \mathrm{E}$ and F). 
A

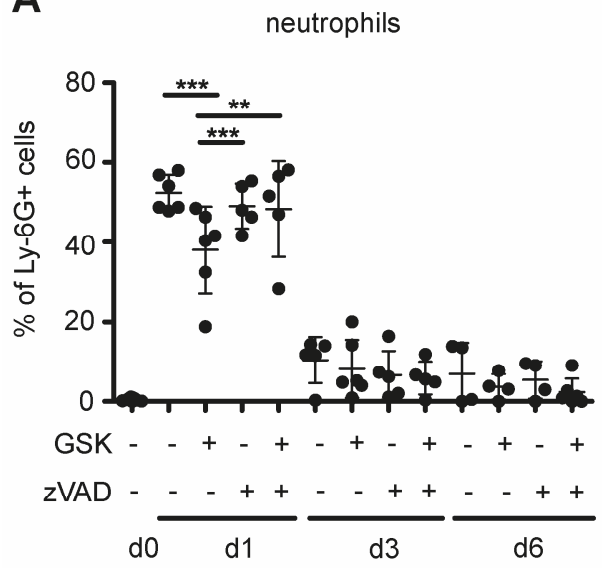

C

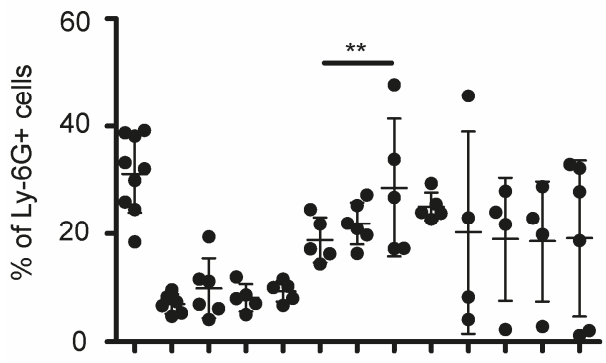

GSK - - + - + + - + + - +

zVAD -

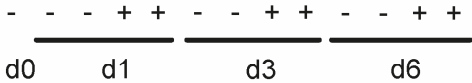

E

macrophages

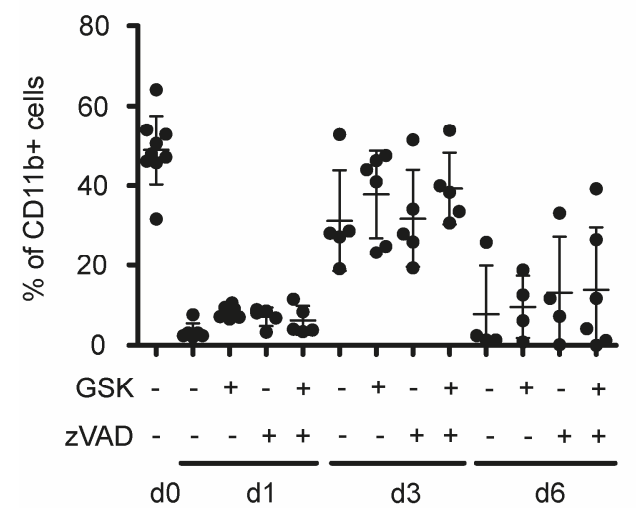

B

neutrophils

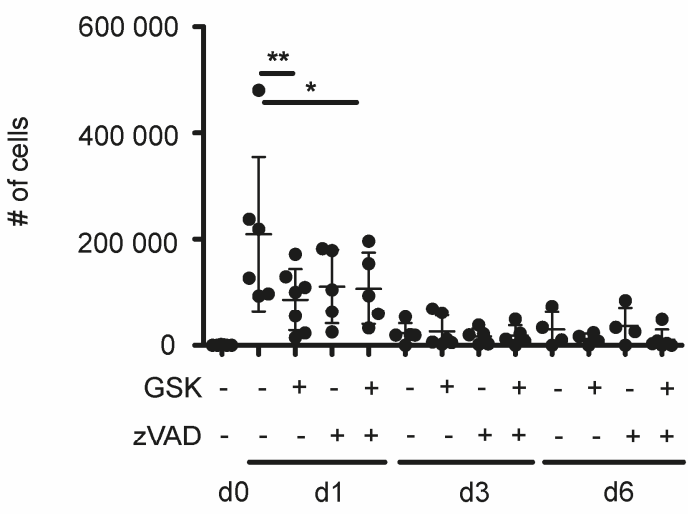

D

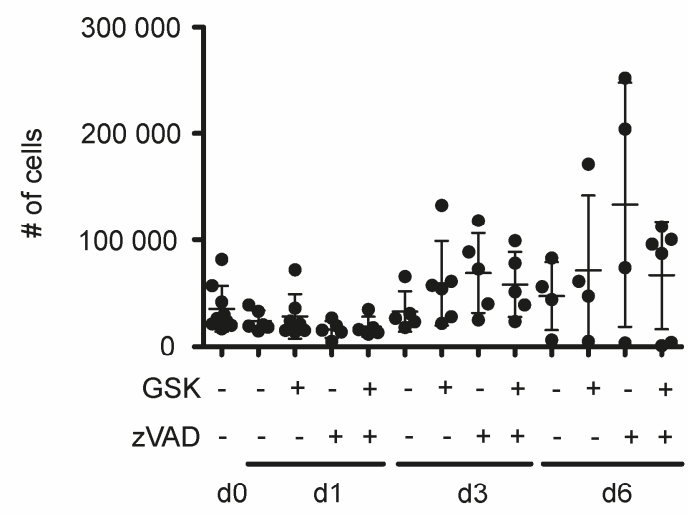

$\mathbf{F}$

macrophages

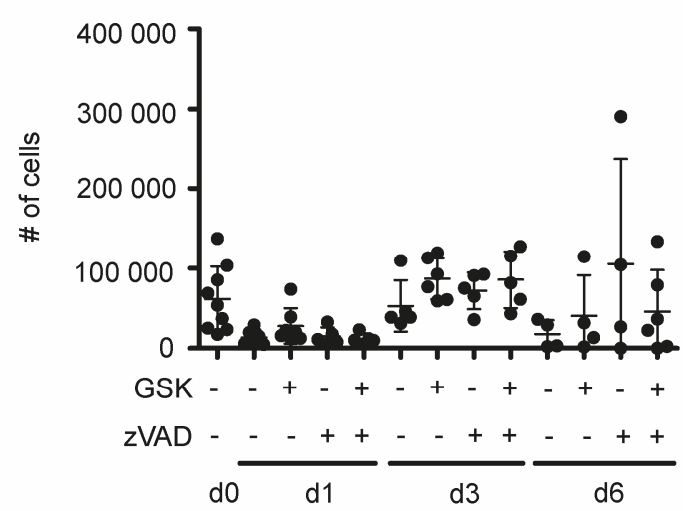

Figure 54. Injection of cell death inhibitors led to changes in immune cell levels. Mice were treated with zVAD.fmk or GSK'872 8 hours after induction of Zymosan-A-induced peritonits and cells of the peritoneum were isolated at day 1,3 and 6 . Either $\%$ of cells or number of cells were analyzed. A.+B. neutrophils, C.+D. B cells, E.+F. macrophages. Data are shown as mean \pm SEM. $n=4-9$.

Injection of dying cells had no significant effect on the composition of immune cells in the peritoneum after Zymosan-A injection compared to control animals (Figure 55). 
A

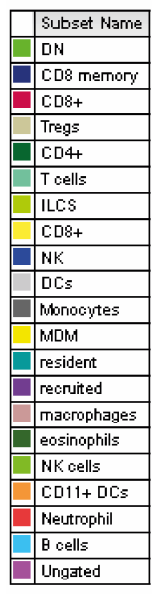

A

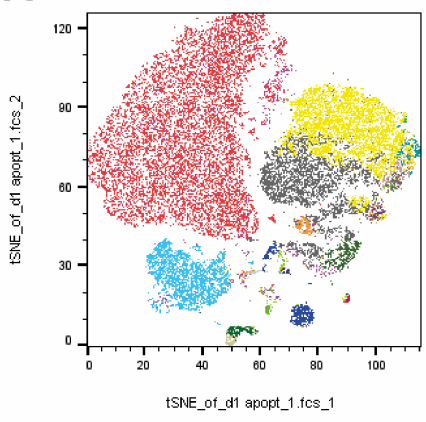

C

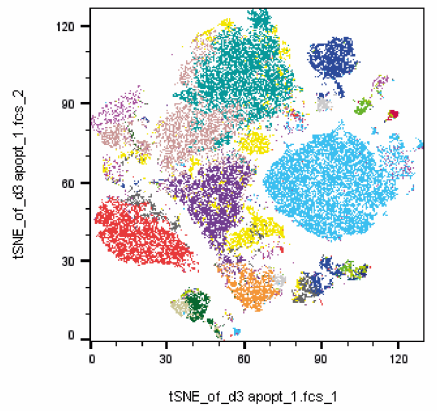

E

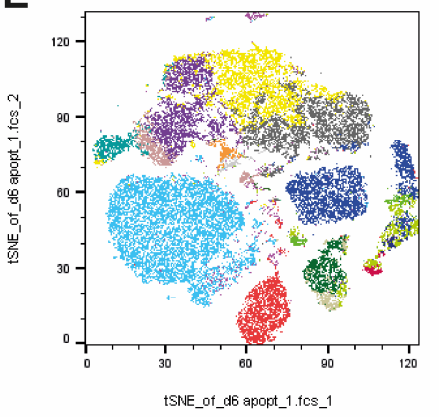

B

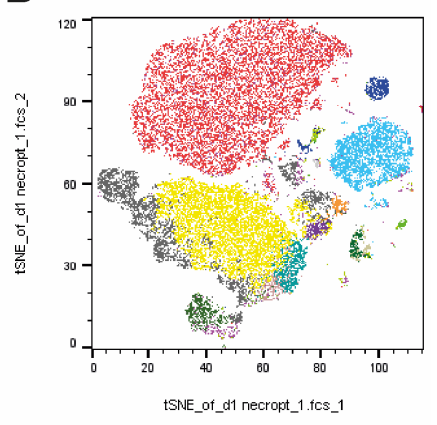

D

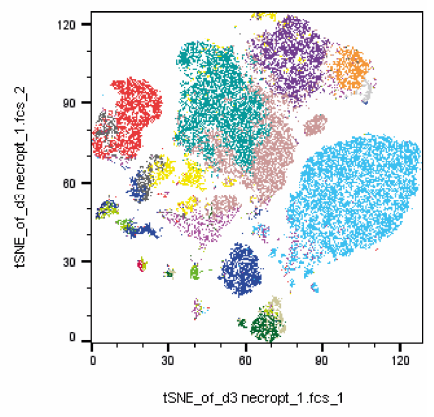

$\mathbf{F}$

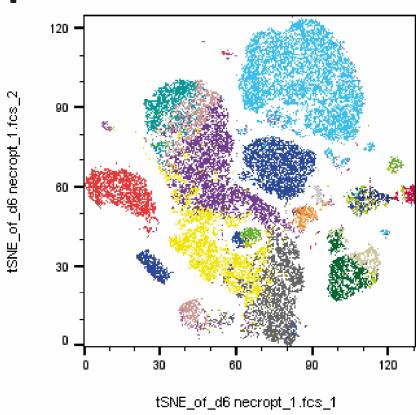

Figure 55. t-SNE maps of animals treated with dying cells show immune cell profiles during Zymosan-A-induced peritonitis. Cells of the peritoneum after injection of apoptotic or necroptotic cells after $1(\mathbf{A}+\mathbf{B}), 3(\mathbf{C}+\mathbf{D})$ or 6 days $(\mathbf{E}+\mathbf{F})$ were isolated and analyzed by staining with different immune cell markers and flow cytometry. After gating, t-SNE analysis was performed. n=3-7.

Further analysis of the immune cells revealed increased numbers of single and CD45 positive cells after injection of dying cells at day 3 (Figure $56 \mathrm{~A}$ and B). Moreover, percentage of neutrophils was significantly reduced after injection of necroptotic cells at day 1 (Figure $56 \mathrm{C}$ ), indicating that any modulation of necroptosis during inflammation reduces neutrophil levels. The numbers of neutrophils were not significantly changed (Figure 56 D). Injection of necroptotic cells led to increased percentage of macrophages in the peritoneum at day 6 (Figure 56 E). Numbers of macrophages were also increased, 
although not significantly (Figure 56 F). Higher levels of macrophages indicated a delayed resolution of inflammation after injection of necroptotic cells. As we saw only minor changes in the immune cell profil after injection of dying cells, we concentrated our research on the inhibition of apoptosis and necroptosis during Zymosan-A-induced peritonitis.

A

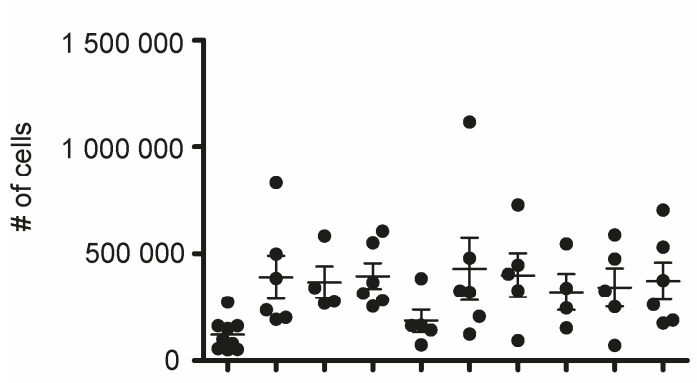

apopt. cells

necropt. cells -

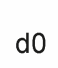

single cells

$+$

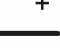

neutrophils

C

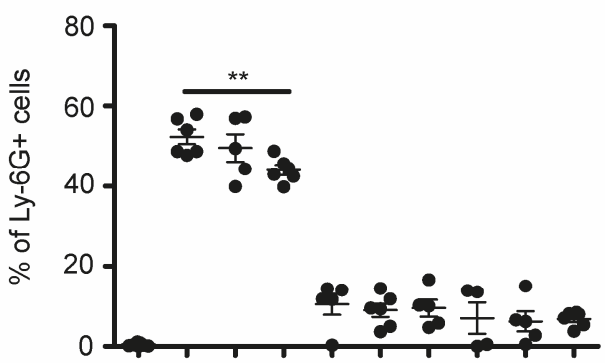

apopt. cells

necropt. cells

E
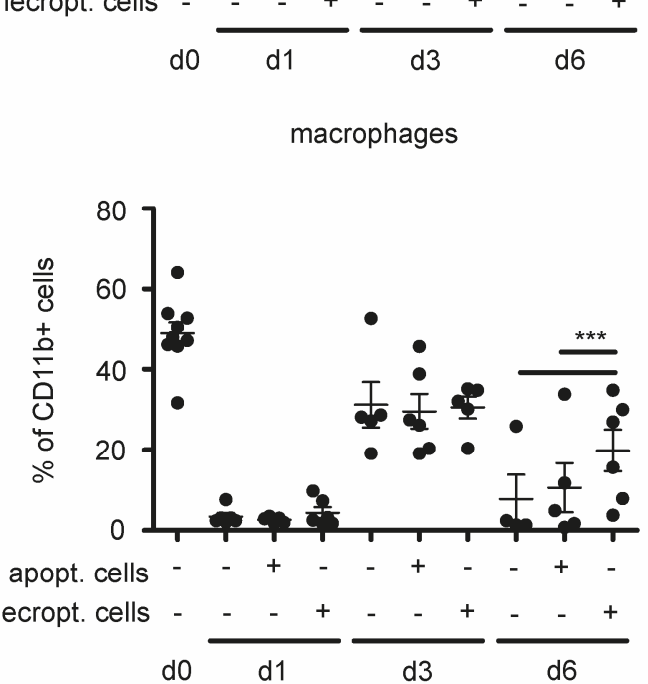

B

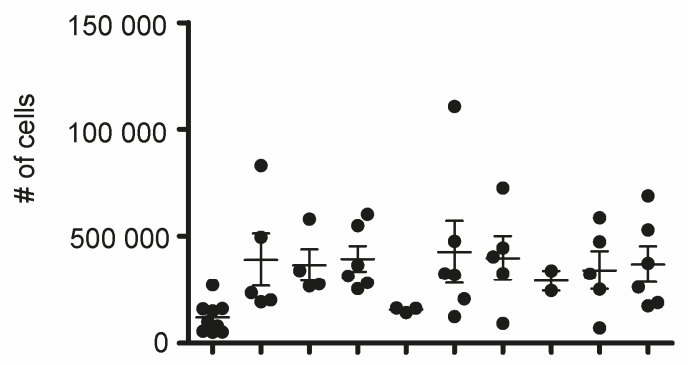

apopt. cells

necropt. cells
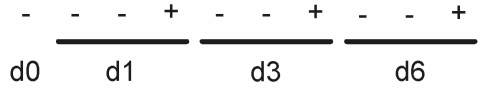

D

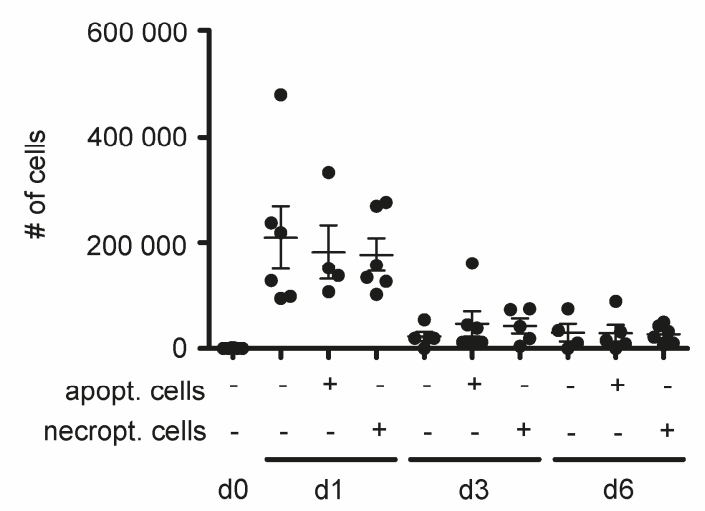

F

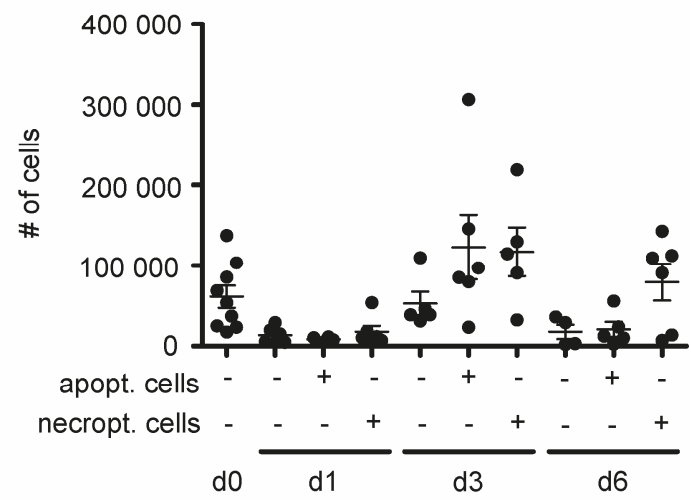

Figure 56. Injection of dying cells led to changes in immune cell levels. Mice were treated with apoptotic or necroptotic cells 8 hours after induction of peritonits and cells of the peritoneum were isolated at day 1 , 3 and 6 . Either $\%$ of cells or number of cells were analyzed. A. single cells, B. CD45+ cells, C.+D. neutrophils, E. $+\mathbf{F}$. macrophages. Data are shown as mean \pm SEM. $\mathrm{n}=4-9$. 
Next, we analyzed cytokines in the peritoneum after injection of cell death inhibitors (Figure 57). As expected, the pro-inflammatory cytokine IL-1 $\beta$ was increased at day 1 and decreased again at day 3 (Figure $57 \mathrm{~A}$ ). Inhibition of apoptosis and necroptosis slighlty increased IL-1 $\beta$ concentrations at day 1 compared to control animals. The antiinflammatory cytokine IL-10 was significantly increased after injection of zVAD.fmk and of zVAD.fmk together with GSK'872 (Figure 57 B). Levels of the pro-inflammatory cytokine TNF- $\alpha$ were increased at day 1 in animals treated with cell death inhibitors, indicating that inhibition of apoptosis and/or necroptosis led to a stronger induction of inflammation.

A

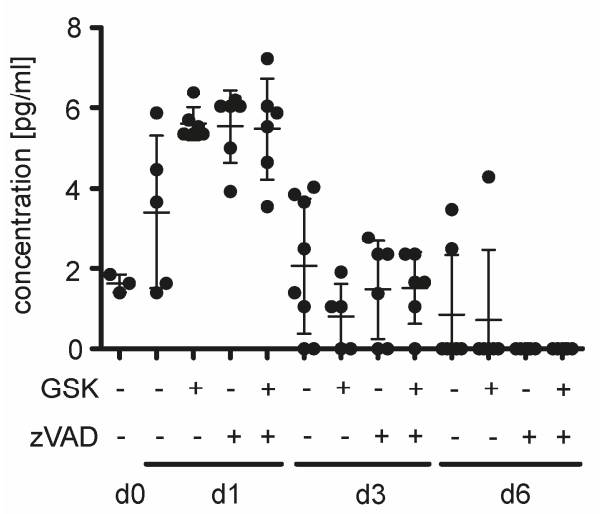

C

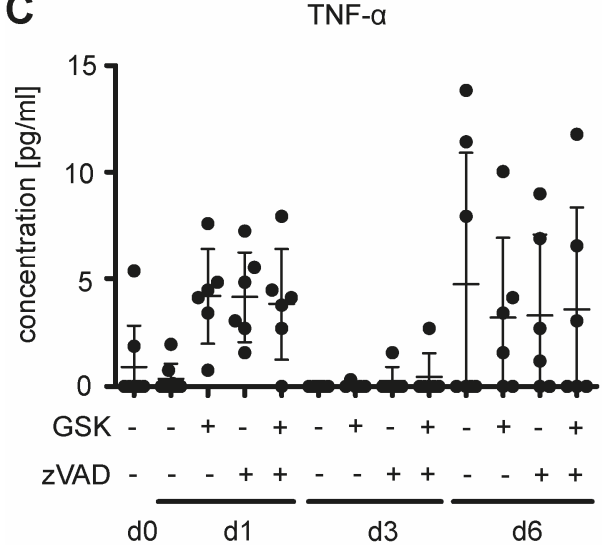

B IL-10

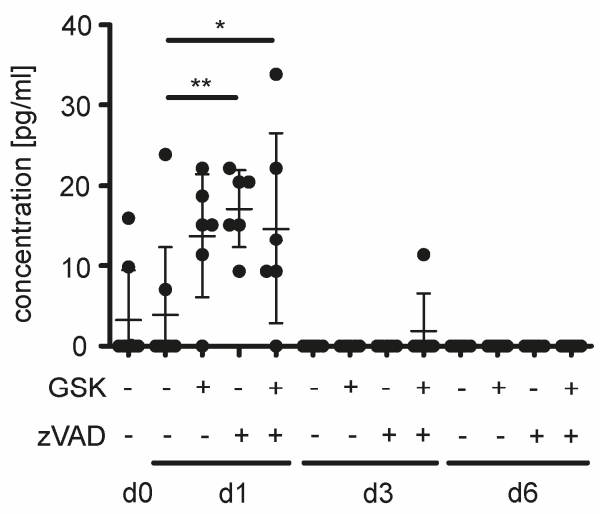

Figure 57. Concentrations of different cytokines in the lavage of mice treated with cell death inhibitors were analyzed. 8 hours after Zymosan-A injection, mice were treated with GSK' 872 and/or zVAD.fmk and after 1, 3 or 6 days conentrations of IL-1 $\beta$ (A.), IL-10 (B.) and TNF- $\alpha$ (C.) in the lavage were measured. Data are shown as mean \pm SEM. $\mathrm{N}=5-8$.

Inhibition of necroptosis led to decreased levels of neutrophils in the peritoneum at day 1 , indicating that the recruitment of neutrophils and therefore chemokine levels were altered. For this reason, 13 different pro-inflammatory chemokines in the lavage were 
analyzed (Figure 58). Only eight of the chemokines could be detected but showed no significant change due to treatment. 
A

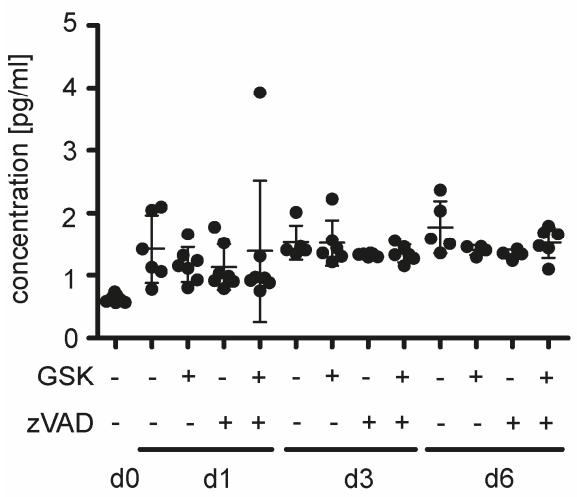

C

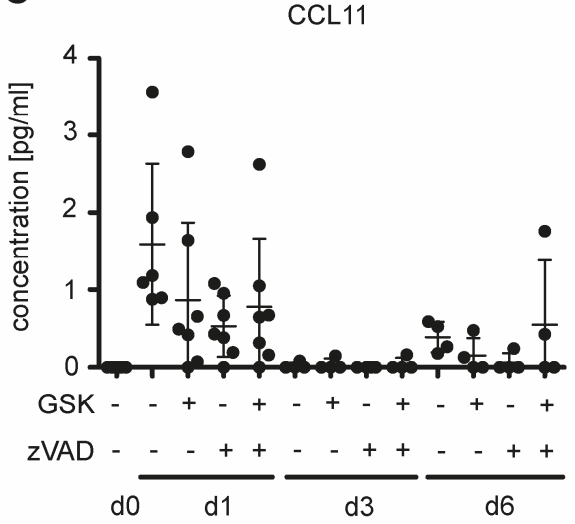

$E$

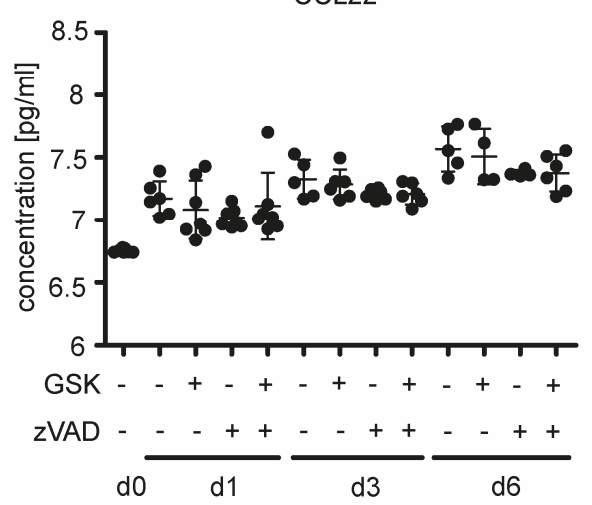

G

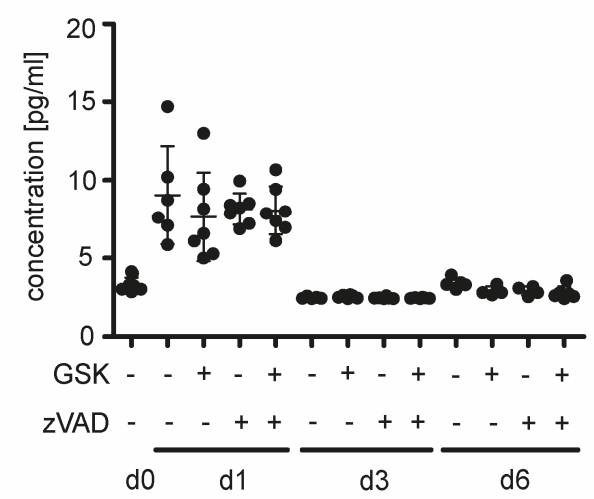

B CCL4

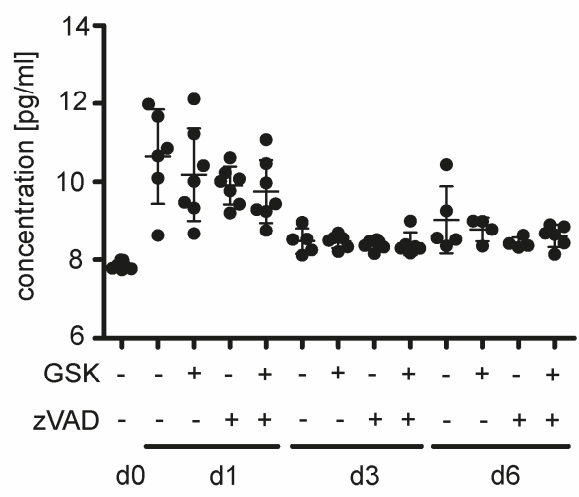

D

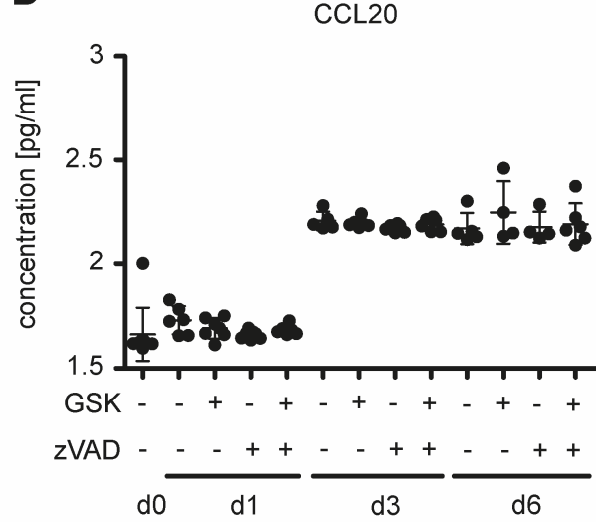

F

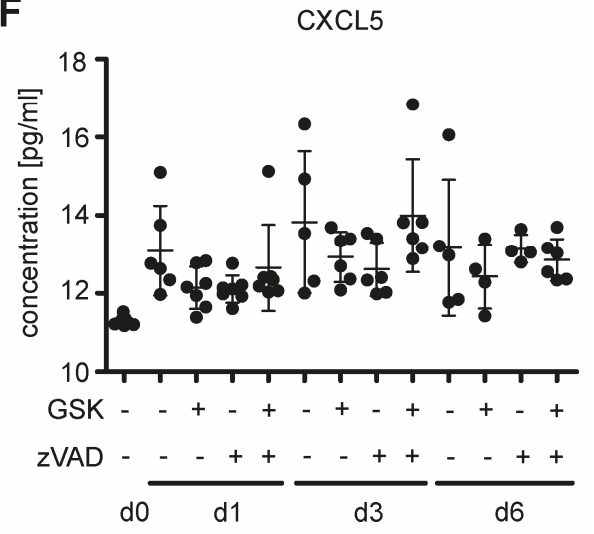

H

CXCL13

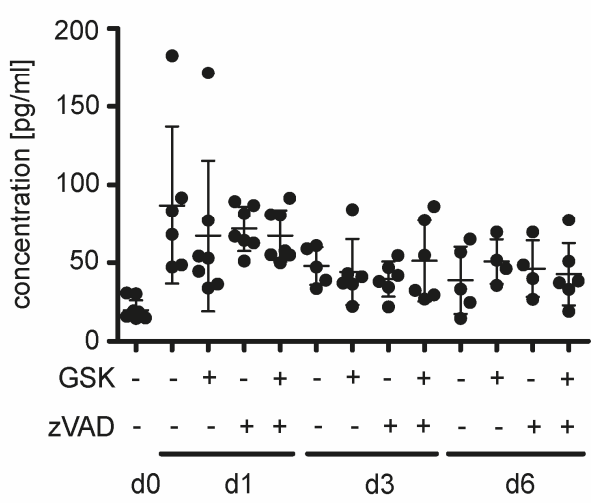

Figure 58. Legend can be found on the next page. 
Figure 58. Concentrations of different chemokines in the lavage of mice treated with cell death inhibitors were analyzed. 8 hours after Zymosan-A injection, mice were treated with GSK' 872 and/or zVAD.fmk and after 1, 3 or 6 days concentrations of CCL2(A.), CCL4 (B.), CCL11 (C.), CCL20(D.), CCL22 (E.), CXCL5 (F.), CXCL9 (G.) and CXCL13 (H.), in the lavage were measured. Data are shown as mean \pm SEM. $n=4-7$.

Concentrations of CXCL1 were below the detection limit. Nevertheless, the fluorescence intensity of CXCL1 detection antibodies is shown in Figure 59. Inhibition of apoptosis and necroptosis led to decreased fluorescence intensity, indicating that less CXCL1 was bound and was present in the peritoneum.

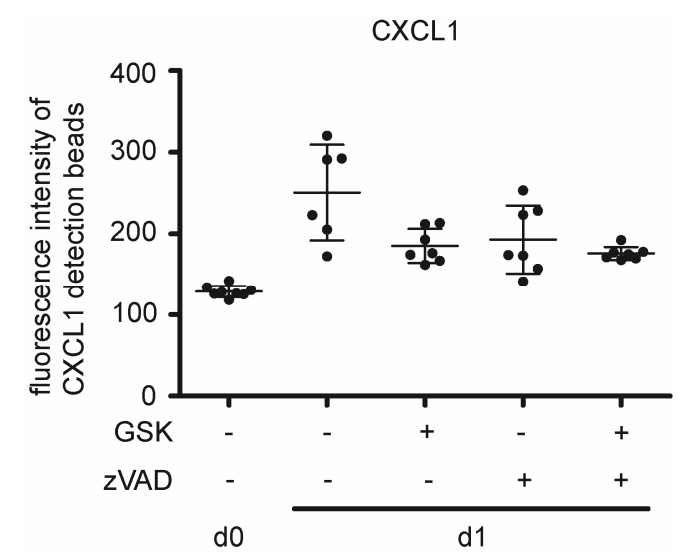

Figure 59. Levels of CXCL1 in the lavage of mice treated with cell death inhibitors were analyzed. 8 hours after Zymosan-A injection, mice were treated with GSK'872 and/or zVAD.fmk and after 1 day levels of CXCLl in the lavage were measured. Data are shown as mean \pm SEM. $n=6-7$.

RNA sequencing revealed proliferation of peritoneal macophages after co-culture with apoptotic cells in vitro. To confirm this finding in vivo, we isolated mRNA of peritoneal macrophages isolated from mice treated with Zymosan-A and zVAD.fmk and analyzed mRNA levels of cell cylce genes (Figure 60). mRNA of Aurka was decreased at day 1 in mice treated with zVAD.fmk (Figure $60 \mathrm{~A}$ ) and zVAD.fmk treatment reduced mRNA levels of Ccnb1 at day 1, 3 and 6 (Figure 60 B). mRNA level of Cdk1 were significantly reduced in mice treated with zVAD.fmk at day 3 (Figure $60 \mathrm{C}$ ). These findings give a first hint that proliferation of macrophages during Zymosan-A induced peritonitis was blocked by inhibition of apoptosis. 
A

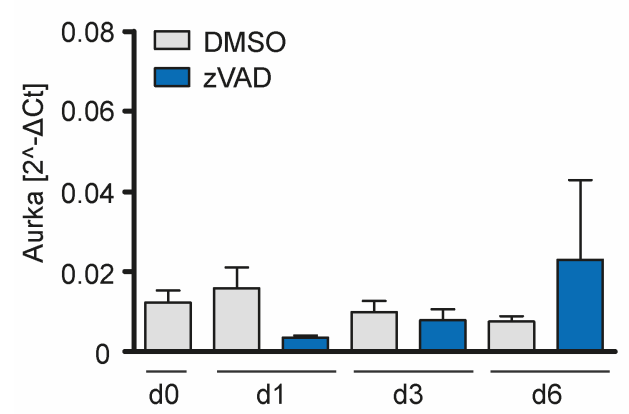

C

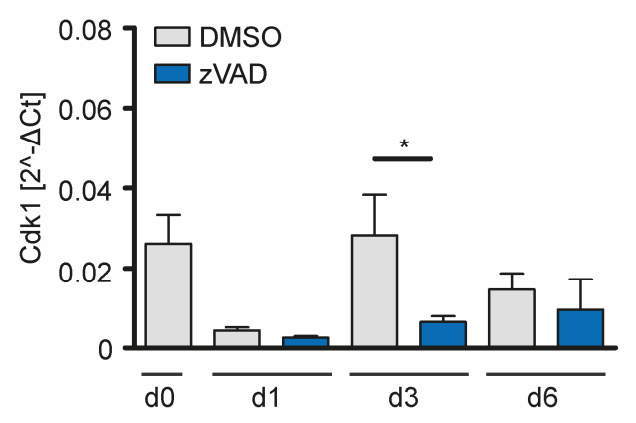

B

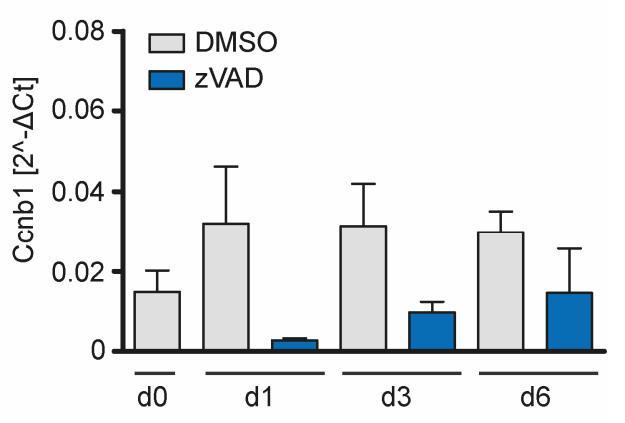

Figure 60. mRNA level of cell cycle genes in macrophages at day 1 of Zymosan-A-induced peritonitis and zVAD.fmk injection. 8 hours after Zymosan-A injection, mice were treated with zVAD.fmk. After 1 day macrophages were seeded in wells and mRNA was isolated. mRNA levels of Aurka (A.), Ccnb1 (B.) and Cdk1 (C.) were analyzed. Data are shown as mean + SEM. n=3-6. 


\section{Discussion}

\subsection{Part I: IFNs induce MLKL expression in cancer cells}

\subsubsection{MLKL is transcriptionally upregulated upon IFN signaling}

The aim of the first part was to study the IFN-induced upregulation of the necroptotic regulator MLKL in different cancer cells. We could show that MLKL is upregulated at mRNA and protein level upon IFN signaling via RT-PCR and Western Blot analysis. Increased levels of MLKL could be a general phenotype of IFN signaling as MLKL was upregulated in several different cancer cell lines and upon treatment with type I and type II IFNs. The transcription factor IRF1, which is activated by IFN signaling, was upregulated prior to MLKL on mRNA and protein level and we speculated that IRF1 drives MLKL expression. This hypothesis was strengthened by Actinomycin D chase experiments, which showed that the upregulation of MLKL is dependent on active transcription. Moreover, a prediction analysis confirmed binding sites of the transcription factor IRF1 and STAT1 in the promoter region of MLKL. Knockdown of IRF1 or STAT1 separately had no effect on MLKL expression after IFN treatment since IRF1 knockdown efficiency was poor, resulting in no loss in MLKL. Simultaneous knockdown of IRF1 and STAT1 inhibited MLKL upregulation. This might indicate that STAT1 and IRF1 work together in inducing MLKL expression after IFN stimulation and are both requiered for MLKL expression. However, IRF1 kockout showed significant decreased MLKL levels, proving that IRF1 is essential to induce MLKL expression. In summary, we could show for the first time, that IRF1 induced expression of MLKL by IFN signaling.

Increased expression of MLKL upon IFN signaling was shown before in several studies mostly as side-finding and in several published datasets of IFN-regulated genes. MLKL was found to be induced at least two-fold by IFN $\beta$ and IFN $\gamma$ together with other kinases like protein kinase $\mathrm{R}$ (PKR) in murine embryonic fibroblasts (MEFs) using DNA microarray analysis. In the same study, Actinomycin D chase experiments prevented IFN $\gamma$-driven RIPK1-RIPK3 formation. Thapa et al. suggested that IFN signaling induces expression of a mRNA by STAT1 promoter binding, which activates latent PKR and results in necroptosis [21]. This finding was confirmed in the setting of WT and RIPK1 knockout MEFs, which showed similar upregulation of MLKL upon IFN treatment but 
not upon TNF or poly I:C treatment [64]. Again in primary MEFs, MLKL protein level was increased after treatment with IFN $\beta$ or IFN $\gamma[65,66]$. In the context of Gaucher's disease, induced by conduritol B epoxide injection into mice, IFN signaling was upregulated. Knockout of IFNAR1 resulted in significant lower MLKL mRNA levels [67]. Furthermore, IFN $\gamma$ was able to induce the expression of MLKL in primary mouse dermal fibroblasts and HT29 colon carcinoma wild type and RIPK1 -/-, Casp8 -/- and TNFR -/- cells [68]. Günther et al. could show for the first time, that MLKL expression is induced by an IFN $\gamma / \mathrm{STAT} 1-$ dependent pathway and confirmed this with a promoter luciferase assay in primary hepatocytes and also provided similar results in human embryonic kidney cells, BNL cells and MEFs. They showed that IFN $\gamma$ is a key driver of liver pathology in a model of inflammation-dependent hepatitis caused by increased MLKL transcription and protein abundance [69]. Here, IRF1 was not studied as activator of MLKL expression. Either, IRF1 does not play a role in MLKL transcription in the context of hepatitis or STAT1 is requiered to activate IRF1 signaling. Taken together, these publications support our work and highlight the importance of IFN-induced upregulation of MLKL expression in several cell lines and upon type I and type II IFN treatment. In addition to the previously described IFN-dependent upregulation of MLKL, we could show for the first time, that increased MLKL expression is mediated by the transcription factor IRF1.

In our experiments, we could not show an influence of IFN signaling on the levels of the other necroptotic mediators RIPK1 and RIPK3. Although RIPK1 was described as an ISG in one study [70], our experiments do not support this finding leading us to the conclusion that IFN signaling regulates necroptosis only by upregulating MLKL expression.

\subsubsection{IFN-dependent upregulation of MLKL can be used to sensitize} apoptosis-resistant cancer cells towards necroptosis

Many cancers are able to block apoptosis by expression of anti-apoptotic proteins or inactivation of pro-apoptotic genes and develop treatment resistance [71]. To overcome this resistance other programmes of regulated cell death can be induced, for example necroptosis. It was shown before that IFN $\gamma$ induces necroptosis together with Smacmimetics, when caspases are inhibited (by zVAD.fmk) in various cancer cell lines [72]. In our setting, treatment of EFM-192A cells with IFN $\gamma$ and the caspase inhibitor zVAD.fmk induced cell death in around $20 \%$ of the cells after 72 hours. These low levels 
of cell death can be explained by the fact that no Smac-mimetic was used and IFN $\gamma$ alone is not able to induce high levels of cell death in these cells. Here, cells are dying via necroptosis upon treatment with IFN $\gamma$ and zVAD.fmk as MLKL was phosphorylated and cell death could be inhibited by the MLKL inhibitor NSA. Furthermore, IFN $\gamma$ was able to reduce cell viability in EFM-192A and HeLa cells. Indeed, IFNs have anti-proliferative effects depending on the context by e.g. targeting several proteins that are involved in cell cycle control [73-76].

The transcription factor IRF1 was shown to be required for Smac-mimetic- and IFN $\gamma$ induced necroptosis [72]. Together with our finding, that IRF1 activates MLKL expression, this suggests that IRF1-mediated upregulation of MLKL is able to promote necroptosis. Sensitizing cells towards necroptosis by upregulation of MLKL was shown in other settings, too. In bone marrow derived macrophages, IFN-dependent MLKL expression enables a rapid necroptotic response. In this setting, constitutive IFN signaling resulted in a critical threshold of MLKL, which helped to quickly induce MLKL oligomerization and necroptosis [77]. Furthermore, MEFs, which are relatively insensitive to TNF and zVAD.fmk-induced necroptosis, could be sensitized to cell death by pre-treatment with IFN $\beta$, showing that an increased expression of MLKL induced by IFNs may also contibute to necroptosis $[64,66]$. In mouse dermal fibroblasts and HT29 cells, MLKL upregulation primed cells for necroptosis by the co-treatment of IFN $\gamma$ and zVAD.fmk [68]. Muendlein et al. showed that type I IFNs downregulate translation of cytokines, which are expressed downstream of RIPK1/RIPK3. They suggested that together with IFN-induced upregulation of MLKL, IFN treatment switches RIPK1/RIPK3-activation from inflammatory towards necroptotic signaling [78].

Our findings in line with the discussed publications could show that upregulation of MLKL by IFNs provide a valuable tool to sensitize cells towards necroptosis. Additionally, this could be helpful to overcome apoptosis resistance in cancer cells. Indeed, IFNs are used as therapeutics to activate the immune system and induce apoptosis in certain cancers, which was described in section 1.6.

In summary, we could show that activation of the IFN pathway in cancer cells leads to upregulation of MLKL on mRNA and protein level. We propose that induction of the JAK-STAT1 pathway and enhanced IRF1 transcription is responsible for MLKL upregulation, which could lead to necroptosis if caspases are inhibited (Figure 61). 


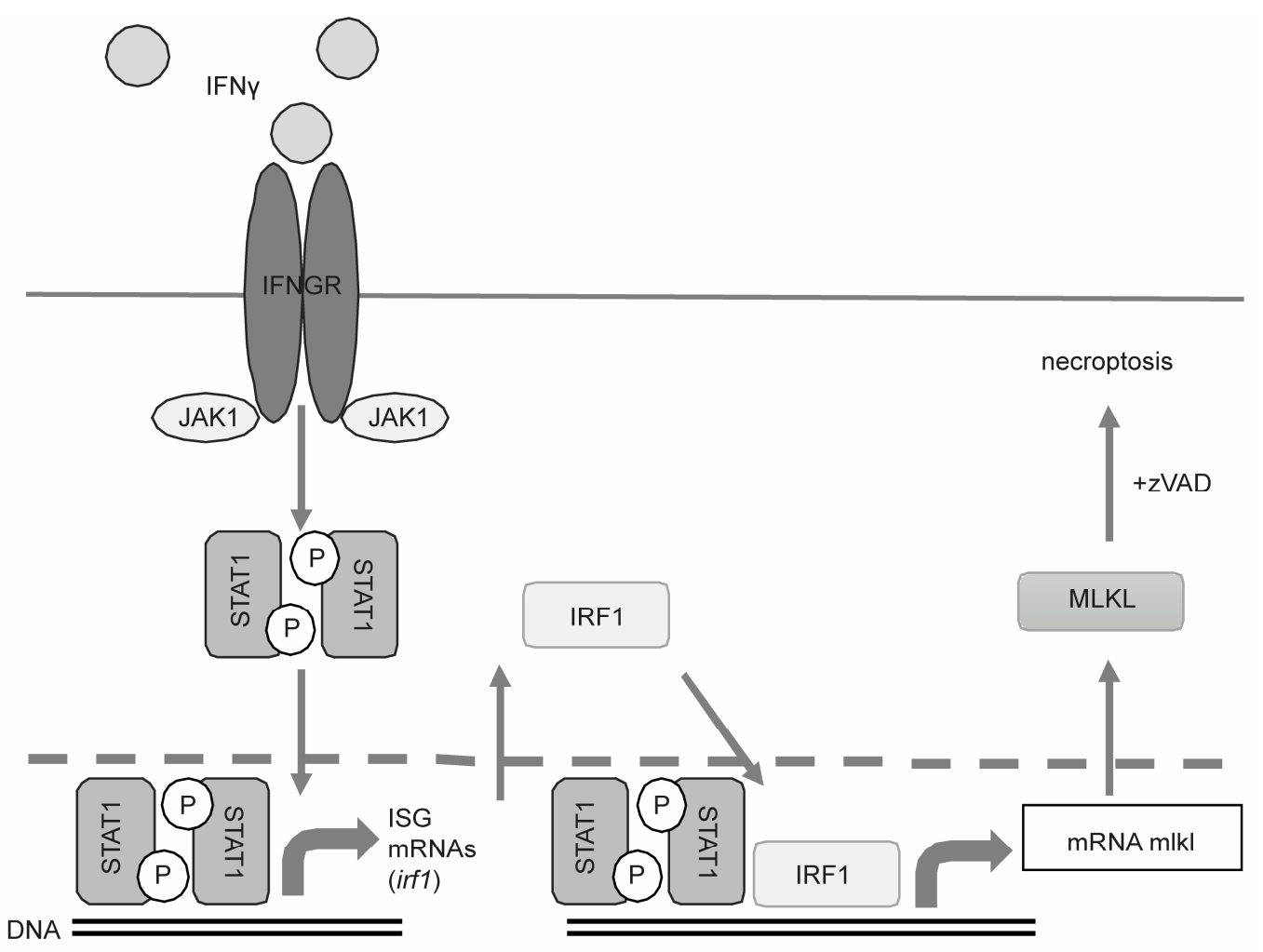

Figure 61. MLKL expression is regulated by IRF1 and STAT1. Binding of IFNs to their IFN receptor induces the activation and phosphorylation of STAT1, which leads to the expression of IRF1. Based on our results, we hypothesize that IRF1 and STAT1 upregulate MLKL transcriptionally. The higher protein expression of MLKL might be able to sensitize cells towards necroptosis.

\subsection{Part II: Effect of dying cells on immune cells and resolution of inflammation}

The aim of the second part was to analyze the effect of apoptotic and necroptotic cells on immune cells. For that we first generated pure cell death systems to avoid activation of signaling pathways other than apoptosis or necroptosis. Next, we established a co-culture system of dying cells and peritoneal macrophages and used this system to analyze the gene expression profile of peritoneal macrophages after co-culture in an unbiased approach. Finally, we studied the effect of dying cells or inhibition of cell death in an in vivo model of self-resolving inflammation.

\subsubsection{Pure cell death systems}

To analyze the effect of apoptosis or necroptosis on immune cells, we wanted to use a pure cell death system, in which only a specific type of programmed cell death is 
activated. In our system apoptosis or necroptosis is induced by transducing NIH 3T3 cells with plasmids containing either human caspase 8 or murine RIPK3 fused to dimerizer domains. Binding of a chemical dimerizer to these domains induces close proximity of two or more proteins leading to proteolytic activation (caspase 8) or auto-phosphorylation (RIPK3) and induction of cell death. Chemical induction of proximity was previously shown to be sufficient for activation of caspase 8 or RIPK3 by dimerization or oligomerization and induce apoptosis or necroptosis, respectively [24-26, 79, 80]. Furthermore, we verfied the induction of apoptosis by activation of caspase 3 or 7, inhibition of cell death with zVAD.fmk and detection of Annexin V positive cells. Induction of necroptosis was verified by detection of activated and phosphorylated MLKL on protein level and inhibition of cell death with GSK'872. The kinetics of dimerizer-induced apoptosis and necroptosis were comparable to the results gained by Yatim et al. [26]. NIH 3T3 cell were used as these cells are easy to handle, easy to transfect and reflect the in vivo situation as they are fibroblasts, which are omnipresent in tissues. Human caspase 8 was transduced into NIH 3T3 cells to distinguish modified and endogenous caspase 8 in these cells. Murine RIPK3 could be used as NIH 3 T3 cells do not express RIPK3 [24].

The used dimerizer is designed to be specific for the modified FKBP domain, FKBP F36V (FV), and does not bind to endogenous FKBP and thereby, does not induce other signaling pathways. The activated death effector proteins, caspase 8 and RIPK3, have also other functions besides activation of cell death. Caspase 8 was shown to be able to activate transcription of inflammatory cytokines without induction of apoptosis [81, 82]. Here, we did not see upregulation of inflammatory cytokines upon activation of caspase 8 . Originally, it was suggested that RIPK1 and RIPK3 cause inflammation by inducing necroptosis but they have additional functions beyond induction of cell death [83]. RIPK3 is important for the control of viral infections and RIPK1 has a crucial role in promoting NF- $\kappa \mathrm{B}$ activation $[82,83]$. Yatim and colleagues showed that activation of RIPK1 by dimerizer-induced oligomerization of RIPK3 results in NF- $\mathrm{BB}$ signaling, which is essential for the immunogenicity of necroptotic cells. Furthermore, they could show that dimerizer-induced necroptosis leads to active transcription and translation of inflammatory cytokines and chemokines like IL-6 and CXCL1, which is dependent on NF- $\kappa B$ signaling [26]. We could confirm this finding, as necroptotic cells showed higher mRNA levels of IL-6 and protein levels of IL-6 and CXCL1. It would be interesting to 
further analyze the necroptosis-independent functions of RIPK3 and elucidate, whether the shown effects of necroptotic cells are dependent only on RIPK3 activation.

\subsubsection{Co-culture of dying cells with peritoneal macrophages}

Next, we established a co-culture system of dying cells and peritoneal macrophages to analyze the effect of dying cells on immune cells. We first tried to use a direct co-culture as it would be the optimal situation to allow direct contact of dying cells and immune cells, but it was technically impossible to separate the cell types after the co-culture. Therefore, we used a transwell system with a pore size of $6.5 \mu \mathrm{m}$, which allowed simple separation of cell types and exchange of apoptotic bodies, cell fragments and mediators across the membrane. Functionality of the co-culture system was proven by analysis of released mediators and polarization markers of the macrophages. The pro-inflammatory chemokine CXCL5 was released in response to apoptotic cells, which was described before in response to apoptotic cancer cells [59]. In this study, bone marrow macrophages phagocytose apoptotic cancer cells, which activates STAT3 and NF- $\kappa$ B pathways. This results in the production of pro-inflammatory cytokines including CXCL5 promoting tumor growth [59]. They also observed an upregulation of other pro-inflammatory mediators like CXCL1 and IL-6 in response to apoptotic cells, which we did not find. We suggest that only CXCL5 is released as response to apoptotic cells in our setting. Furthermore, we used a different type of macrophage (peritoneal macrophages instead of bone marrow-derived macrophages) and used a pure apoptotic system instead of inducing apoptosis by UV light, which could lead to slightly different results.

Upon co-culture with necroptotic or apoptotic cells, polarization markers of macrophages were altered. Efferocytosis of apoptotic cells is believed to polarize macrophages towards an anti-inflammatory M2 phenotype, whereas necroptotic cells induce pro-inflammatory signaling [84]. On the one hand, we could confirm, that the co-culture of macrophages with necroptotic cells resulted in higher levels of the pro-inflammatory cytokine IL-6, but on the other hand, no clear M1 or M2 polarization could be concluded as mRNA levels of anti-inflammatory markers CD206 and ALOX15 were also upregulated in macrophages co-cultured with necroptotic cells. Yatim and colleagues could show that necroptotic cells induced maturation of dendritic cells by upregulation of activation markers, too [26]. This confirms that phagocytosis of necroptotic cells influences immune cells. As macrophages exist in a spectrum of populations, the M1/M2 classification does 
not reflect all different subsets of macrophages. Up- and down-regulation of polarization markers showed that dying cells and peritoneal macrophages are able to interact in the used transwell system.

\subsubsection{Apoptotic cells induce proliferation of peritoneal macrophages}

To gain new insights into the genetic profile of macrophages in response to dying cells, we used an unbiased approach by performing RNA sequencing of mRNA isolated from macrophages co-cultured with apoptotic or necroptotic cells. In most studies the direct comparison of apoptotic or necroptotic cells is missing or no pure cell death system is used, suggesting that with our results it will be possible to directly compare the effects of apoptotic or necroptotic cells on peritoneal macrophages for the first time.

Analysis of RNA sequencing data revealed significant differences in gene expression of macrophages co-cultured with apoptotic cells compared to macrophages co-cultured with necroptotic cells or compared to control cells. Interestingly, co-culture with apoptotic cells resulted in activation of the $\mathrm{PGE}_{2}$ pathway together with enrichment of cell cycle, cell division and replication gene sets. $\mathrm{PGE}_{2}$, which is synthesized from arachidonic acid by cyloocygenase enzymes, is the most abundant prostaglandin in the human body and its activation can have homeostatic, inflammatory or anti-inflammatory effects, depending on the context $[85,86]$. Moreover, $\mathrm{PGE}_{2}$ is decribed to be able to induce wound healing and proliferation of stem and progenitor cells. $\mathrm{PGE}_{2}$ can be synthesized in response to caspase 3 and 7 activation, whereas knockout of caspase 3 and 7 leads to deficient wound healing and liver regeneration in mice [87]. This indicates that $\mathrm{PGE}_{2}$ activation and signaling may be a promissing candidate to induce macrophage proliferation in response to apoptotic cells. Sergeeva and colleagues showed that murine splenocytes start to proliferate in response to low levels of $\mathrm{PGE}_{2}$, but $\mathrm{PGE}_{2}$ had suppressive effects at higher concentrations [88]. Furthermore, proliferation of IL-4activated macrophages was reduced by co-stimulation with $\mathrm{PGE}_{2}$, which regulated the transcription of cell cycle genes and changed the metabolism and polarization of the macrophages [89]. In addition, proliferation of alveolar macrophages was shown to be inhibited by $\mathrm{PGE}_{2}$ [90]. In summary, the effect of $\mathrm{PGE}_{2}$ on proliferation seems to be dependent on the context, polarization and concentration.

We validated the activation of the cell cycle in peritoneal macrohages upon co-culture with apoptotic cells by two different proliferations assays and upregulation of cell cycle 
mediators at mRNA level. Co-culture of peritoneal macrophages with living fibroblasts revealed a beneficial effect of living cells for peritoneal macrophages in the confluence assay as macrophages cultured alone died soon after starting measurements. This beneficial effect could not be shown in IC-21 cells using efluor 670 proliferation assay as this assay was measured using flow cytometry and dead cells were excluded from the analysis. Moreover, IC-21 cells are immortalized peritoneal macrophages indicating a higher replicative and survival potential. This indicates that living fibroblasts produce and release mediators that ensure the survival of peritoneal macrophages ex vivo. The significant increase in proliferation of peritoneal macrophages during co-culture with apoptotic cells could be seen in both experiments indicating that proliferation was induced by released mediators, apoptotic bodies or phagocytosis of apoptotic cells. Necroptotic cells did not influence the proliferation of peritoneal macrophages, demonstrating that this effect is specific for apoptotic cells.

Next, we modulated immune reactions during Zymosan-A-induced peritonits by injection of apoptotic or necroptotic cells or cell death inhibitors. The Zymosan-A-induced peritonitis is an established model to study self-resolving inflammations. Moreover, cells and mediators are easy to isolate at the end of the experiments. The injected cells were dying as we confirmed by Annexin-PI-stainings. Injection of dead cells did not change the ratio of dead cells in the peritoneum. This could be due to dying cells being phagocytosed quickly by macrophages and dendritic cells since phagocytosis of necroptotic cells was recently described to be as fast as phagocytosis of apoptotic cells [91]. Even though injection of dying cells showed promising results in previous studies $[26,29,92]$, we could not detect any significant changes in cytokine and chemokine levels or polarization of macrophages by injecting apoptotic or necroptotic cells during Zymosan-A-induced peritonitis (data not shown). It is possible that numbers of injected cells were too low or other time points would have better reflected the effect of dying cells on the resolution of inflammation.

Interestingly, mRNA levels of cell cycle mediators were reduced upon inhibition of apoptosis by zVAD.fmk in peritoneal macrophages during Zymosan-A-induced peritonitis. However, macrophage levels were not changed upon treatment with zVAD.fmk in vivo. A possible proliferation of macrophages could be overshadowed by the massive influx of monocytes, which occurs during inflammation (see Figure 50). Reduction of dead cells in the lavage of mice treated with cell death inhibitors revealed that inihibition of apoptosis and necroptosis was efficient. However, inhibition of 
apoptosis did not influence levels of neutrophils or macrophages and did not change the timeline of inflammation concluding it had no effect on resolution of inflammation, even though apoptosis of neutrophils is necessary to resolve inflammation. Probably, the chosen concentration of zVAD.fmk was not able to inhibit apoptosis completely. In addition, zVAD.fmk was injected 8 hours after initiation of inflammation and might not able to block apoptosis of neutrophils at that point, which are the main cell type undergoing apoptosis during resolution of inflammation. Interestingly, levels of B cells were increased upon inhibition of apoptosis at day 3 of the Zymosan-A-induced peritonitis. In vitro, in the co-culture of peritoneal macrophages with apoptotic cells, levels of CXCL13 were slightly increased indicating that apoptotic cells could induce secretion of CXCL13 and by that recruitment of B cells $[93,94]$.

Our observation that macrophages are able to proliferate is in line with recent publications. The historical view that macrophages only originate from infiltrating monocytes was refuted by lineage tracing studies showing that macrophages from embryonic sources are able to sustain their numbers by local proliferation [95-98]. Proliferation of macrophages was not only described under physiological conditions but also during inflammation, infection and in diseases like atherosclerosis, obesity, and cancer [99, 100]. Nevertheless, the deatiled molecular mechanisms leading to proliferation of macrophages are not known yet. The growth factor M-CSF could play an important role, as M-CSF was able to promote proliferation of peritoneal macrophages in steady state and during Zymosan-A-induced peritonits [101]. Furthermore, phagocytosis of apoptotic cells could also be an important factor in inducing proliferation of macrophages. Bosurgi and colleagues showed that the genetic ablation of phagocytotic receptors MerTK and AXL inhibited proliferation of macrophages and reduced the induction of anti-inflammatory and tissue repair genes together with IL-4 or IL-13 during helminth infection in lungs or during colitis in the gut of mice [102]. Phagocytosis of polystrene beads, live or heat-killed C. neoformans increased ratio of macrophages in the S phase of the cell cycle [103]. Despite the fact that mechanisms of bacterial phagocytosis and efferocytosis differ fundamentally, these results indicate that phagocytosis stimulates the progression of the cell cycle in macrophages. The phagocytosis-induced proliferation of macrophages may also be dependent on the context and tissue since phagocytosis of apoptotic cells inhibited the cell cycle entry in peritoneal macrophages isolated from thioglycolate challenged mice [104]. Here, apoptosis was induced by irradiation or 
staurosporine treatment and no pure cell death system was used, which is why our data cannot be compared with this publication. In summary, we propose that phagocytosis of apoptotic cells induces signaling pathways, which result in proliferation of macrophages. On the one hand, proliferation of macrophages may be a side effect of caspase-induced proliferation, which is necessary to regenerate tissue loss by division of surviving cells $[105,106]$. On the other hand, elevated numbers of macrophages may be benefical for the removal of dead cells, tissue regeneration and resolution of inflammation. Moreover, proliferation may help to ensure the local population of macrophages after inflammations or infections.

However, disregulation of macrophage proliferation or high numbers of macrophages can contribute to the development of diseases like chronic inflammation, atherosclerosis or fibrosis [107-109]. Additionally, in adipose tissue excess macrophages can induce inflammation [100]. Apoptotic cells are also present in tumors and are phagocytosed by tumor-associated macrophages (TAMs), which also showed self-renewal capacities [110]. Efferocytosis by TAMs can promote growth of the tumor by a pro-tumor polarization of TAMs. The effect of efferocytosis-induced proliferation of macrophages in these diseases needs to be studied further to develop new therapeutic strategies.

\subsubsection{Necroptotic cells change the chemokine profile of macrophages and} recruitment of neutrophils

RNA sequencing revealed upregulation of hypoxia and chemotaxis pathways in macrophages co-cultured with necroptotic cells. The hypoxia pathway is activated by stabilization of HIF-1 $\alpha$ under limited oxygen levels [111]. Stablization of HIF-1 $\alpha$ in macrophages results in M1 polarization, enhanced glycolysis and decreased mitochondrial respiration. To analyze metabolic changes in macrophages, we performed a Seahorse assay. Indeed, macrophages co-cultured with necroptotic cells showed decreased levels of maximal mitochondrial respiration compared to macrophages cocultured with living fibroblasts, indicating that these macrophages had a proinflammatory polarization. However, levels of glycolysis were not changed and mRNA expression of HIF-1 $\alpha$ target genes was not altered. Therefore, further studies are needed to draw an accurate conclusion.

To validate changes in chemotaxis we analyzed chemokine levels in the supernatant after co-culture with necroptotic cells and could show that levels of the neutrophil chemoattractant CXCL1 were increased, whereas levels of B cell chemoattractant 
CXCL13 were decreased. However, concentrations of CXCL13 and levels of B cells were not changed in vivo. The effect of necroptosis on CXCL13 expression or secretion by macrophages was maybe too small to influence recruitment of B cells or CXCL13 levels in the peritoneum of mice.

CXCL1 concentration was also elevated in the supernatant of necroptotic cells, indicating that necroptosis induces the secretion of CXCL1. As described above, Yatim and colleagues saw a similar increase of CXCL1 concentrations in the supernatant of necroptotic cells and concluded that necroptotic cells release pro-inflammatory CXCL1 [26]. However, in our experiments, mRNA level of CXCL1 was increased in macrophages co-cultured with necroptotic cells as well. Furthermore, during our in vivo model of Zymosan-A-induced peritonitis, injection of cell death inhibitors decreased levels of CXCL1. Simultaneously, levels of neutrophils were significantly decreased and weight of mice was faster increased after injection of the necroptosis inhibitor GSK'872. Taken together these results indicate that necroptotic cells secrete CXCL1, induce expression of CXCL1 in macrophages and influence the chemotaxis of neutrophils during inflammation.

In summary, we could demonstrate, that apoptotic cells induce transcription of cell cycle genes and proliferation of macrophages and necroptotic cells are able to influence the chemokine profile of macrophages and thereby the recruitment of neutrophils (Figure 62).

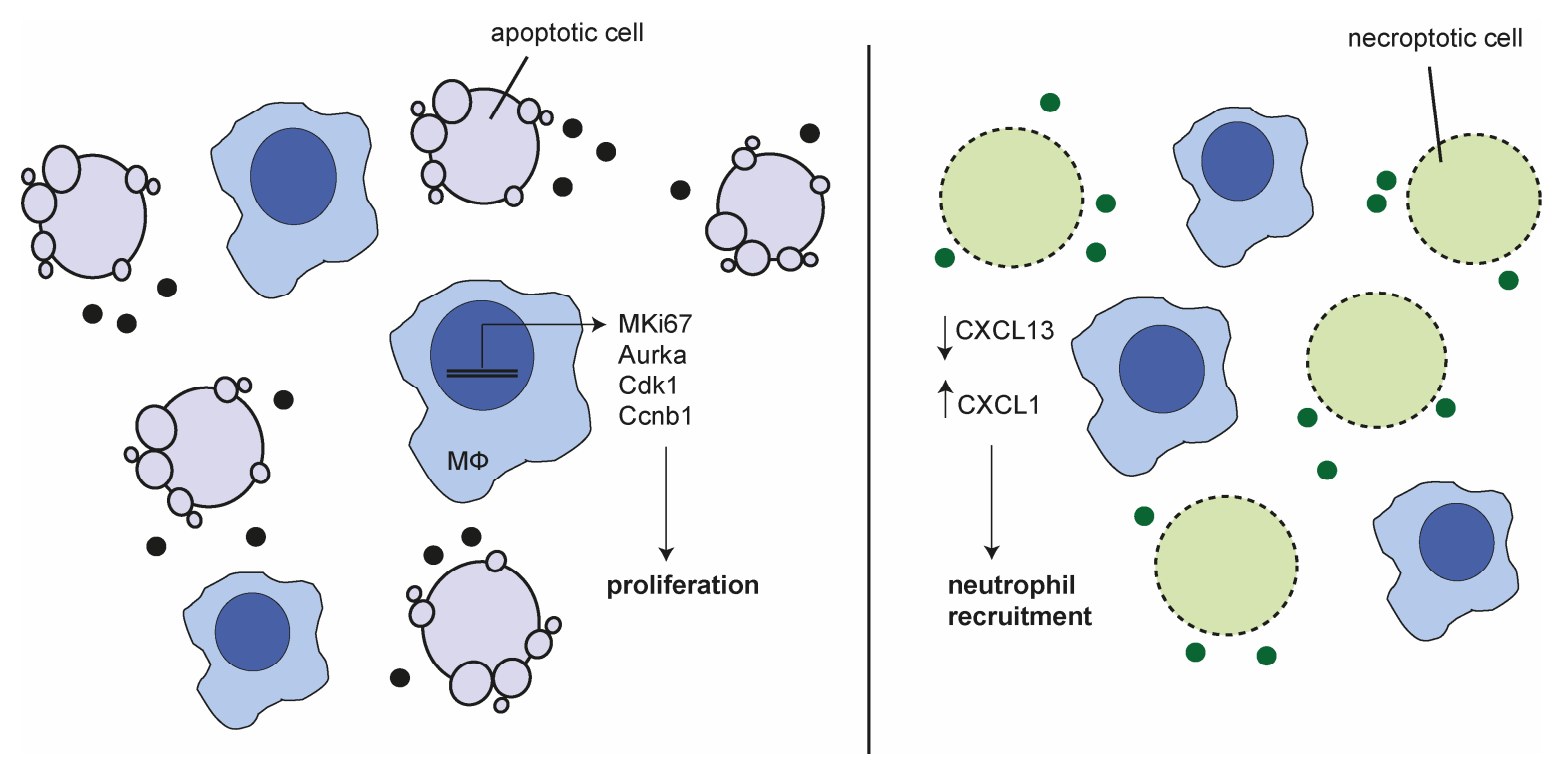

Figure 62. Apoptotic cells induce proliferation of macrophages, whereas necroptotic cells influence the recruitment of neutrophils. 


\subsection{Limitations and Outlook}

The first part of this study highlighted the IFN-dependent upregulation of MLKL. This upregulation was shown to be dependent on IRF1, however a promoter binding assay is missing. Therefore, we could perform a luciferase assay to prove binding of IRF1 to the promoter of MLKL. We demonstrated that MLKL was upregulated upon IFN treatment in various cancer cell lines. It would be useful to confirm MLKL upregulation on mRNA and protein level in healthy cells and in vivo, too. Furthermore, our studies were limited to type I and type II IFNs. Further experiments using treatment with IFN $\lambda$ will evaluate type III IFN dependent regulation of MLKL.

In the second part of this study, we analyzed the effect of apoptotic and necroptotic cells on peritoneal macrophages. As many studies compare apoptosis with necrosis, it would be interesting to analyze immune responses of macrophages to necrotic cells and compare the results to former studies. In the used co-culture system no direct contact of cells was enabled, so that observed effects are based on released mediators or phagocytosis of cell fragments and apoptotic bodies. To distinguish between mediator or phagocytosisinduced effects, macrophages may be directly co-cultured with dying cells.

The genetic profile of macrophages co-cultured with dying cells was analyzed in an unbiased approach using RNA sequencing. Additionally, RNA sequencing of macrophages co-cultured with living cells or using a second single clone of NIH 3 T3 cells or using more time points would affirm our findings. Validation experiments of the RNA sequencing results showed that apoptotic cells induce proliferation of macrophages. The molecular drivers of macrophages proliferation are still unclear and further functional studies are requiered. Knockdown and knockout of $\mathrm{PGE}_{2}$ may reveal that macrophages proliferation is induced by $\mathrm{PGE}_{2}$ activation and signaling.

In our pure cell death system necroptosis was induced by dimerization of RIPK3. It would be interesting to further analyze the necroptosis-independent functions of RIPK3 and if the shown effects of necroptotic cells could be dependent only on RIPK3 activation. Furthermore, RIPK1-independent effects of necroptosis could be analyzed by using RIPK3 with RHIM domain deletion. Necroptotic cells changed the chemokine profile and influenced the recruitment of neutrophils. It would be interesting to prove reduced recruitment of neutrophils upon inhibition of necroptosis during inflammations in vivo. 


\section{Summary (Deutsche Zusammenfassung)}

Programmierter Zelltod ist in fast jeder mehrzelligen Lebensform im Tierreich zu finden. Ein homöostatisches Gleichgewicht zwischen Proliferation und Zelltod muss aufrecht erhalten werden, da eine unkontrollierte Zellteilung $\mathrm{zu}$ Krebs führen kann und ein übermäßiger Zelltod bei Krankheiten wie Alzheimer, Parkinson oder rheumatischer Arthritis $\mathrm{zu}$ finden ist. In der Vergangenheit wurden verschiedene Arten des programmierten Zelltods beschrieben, wobei wir uns in dieser Arbeit auf Apoptose und Nekroptose fokussierten. Apoptose ist der am besten untersuchte Weg des Zelltods und Apoptoseresistenz ist charakteristisch für Krebszellen. Der apoptotische Signalweg wurde erstmals in Caenorhabitis elegans beschrieben und führt $\mathrm{zu}$ deutlichen morphologischen Veränderungen, wie dem Schrumpfen der Zelle, Kondensieren des Chromatins im Zellkern, Membran Blebbing und Fragmentierung des Zellkerns, während die Plasmamembran intakt bleibt und kein zytosolisches Material in das umgebende Gewebe abgegeben wird. Apoptotische Zellen werden von Makrophagen erkannt und phagozytiert, sodass sekundäre Nekrose und damit verbundene Entzündungsreaktionen vermieden werden. Die Induktion der Apoptose führt zur Aktivierung von Cysteinproteasen, welche Caspasen genannt werden. Die Caspasen können sowohl durch den intrinsischen und als auch den extrinsischen Apoptosesignalweg aktiviert werden.

Um die Apoptoseresistenz in Krebszellen zu überwinden, kann Nekroptose als alternativer Mechanismus des programmierten Zelltods induziert werden. Es gibt verschiedene Arten der regulierten Nekrose, welche zur Schwellung der Organellen, Platzen der Plasmamembran, Zelllyse und Freisetzung von intrazellulären Komponenten in die Umgebung führen. Nekroptose ist neben anderen Wegen der regulierten Nekrose wie Ferroptose, Pyroptose und NETose der am besten beschriebene Weg der regulierten Nekrose. Sowohl die extrinsische Apoptose als auch Nekroptose beginnen mit der Ligation von Todesrezeptoren. Als Backup-Mechanismus für die Apoptose wird die Nekroptose nur aktiviert, wenn Caspase 8 inhibiert ist oder nicht exprimiert wird. Nekroptose wird durch Phosphorylierung und Aktivierung der Rezeptor-interagierenden Serin/Threonin-Proteinkinase 1 (RIPK1), RIPK3 und der Pseudokinase mixed lineage kinase domain-like (MLKL) induziert. Phoshoryliertes MLKL oligomerisiert und transloziert zur Plasmamembran, was zum Einstrom von Kalziumionen, zur Produktion von reaktiven Sauerstoffspezies und zur Perforation der Plasmamembran führt. Durch die 
Freisetzung von intrazellulärem Material in die Umgebung ist Nekroptose ein entzündungsfördernder Zelltodweg.

Interferone (IFN) sind Zytokine, welche zusammen mit Smac-Mimetics unter Hemmung von Caspasen Nekroptose in Krebszelllinien auslösen können. Darüber hinaus spielen IFN eine wichtige Rolle bei der Immunantwort gegen Tumore. Nach Bindung von IFN an ihre Rezeptoren wird der JAK/STAT-Signalweg aktiviert, welcher die Expression von IFN-stiumlierten Genen (ISG) induziert. Der genaue Mechanismus wie Interferone in Krebszellen Nekroptose auslösen ist bisher noch nicht vollständig geklärt. Im ersten Teil dieser Arbeit konnten wir in verschiedenen Krebszelllinien MLKL als ISG identifizieren. Die Behandlung von Krebszelllinien verschiedener Tumorentitäten (Brustkrebs, Gebärmutterhalskrebs, kolorektales Adenokarzinom und Leukemie) mit IFN $\alpha,-\beta$ oder $\gamma$ führte zu erhöhten Proteinleveln von MLKL. Da sowohl Typ-I als auch Typ-II IFN die Expression von MLKL erhöhten, scheint es sich um ein generelles Merkmal des Interferonsignalwegs zu handeln. MLKL wurde durch IFN $\gamma$ nicht nur auf Proteinebene sondern auch auf mRNA Ebene hochreguliert, was durch RT-PCR Analysen gezeigt werden konnte. Dies beweist, dass MLKL transkriptionell erhöht wird. In der Tat wurde nach Hemmung der Transkription durch Zugabe von Actinomycin D die MLKLHochregulierung inhibiert. Sowohl der Knockdown der durch IFN aktivierten Transkriptionsfaktoren IRF1 und STAT1 als auch der Knockout von IRF1 verminderten die MLKL-mRNA Hochregulierung signifikant, was zeigt, dass STAT1 und insbesondere IRF1 notwendig sind, um die MLKL-Expression zu induzieren. Dieser erste Teil der Arbeit zeigt, dass die Hochregulierung von MLKL durch IFN in der Lage ist, Zellen für die Nekroptose zu sensibilisieren und es damit möglich sein kann die Apoptoseresistenz in Krebszellen zu überwinden.

Apoptose ist bekanntlich eine entzündungshemmende und anti-inflammatorische Art des programmierten Zelltods, während Nekroptose zur Freisetzung von danger-associated molecular patterns (DAMPs) führt und entzündungsfördernd ist. Die Efferozytose apoptotischer Zellen durch Makrophagen führt $\mathrm{zu}$ einem Umschalten der Makrophagenpolarisation, die die Auflösung der Entzündung begünstigt. Makrophagen sind ein Teil des angeborenen Immunsystems und kommen in jedem Gewebe vor. Sie haben mehrere wichtige Funktionen wie die Phagozytose von Pathogenen, infizierten oder toten Zellen und die Präsentation von Antigenen. Auf verschiedene Umweltreize hin sind Makrophagen dazu in der Lage, unterschiedliche Proteine und Zytokine zu 
exprimieren, was als Aktivierung oder Polarisierung von Makrophagen bezeichnet wird. Historisch werden klassisch aktivierte, pro-inflammatorische M1-Makrophagen und alternativ aktivierte, anti-inflammatorische M2-Makrophagen mit verschiedenen Funktionen unterschieden. M1-Makrophagen produzieren höhere Mengen an proinflammatorischen Zytokinen und Chemokinen. M2-Makrophagen sind potente Phagozyten, haben ein anti-inflammatorisches Zytokinprofil und rekrutieren Th2, regulatorische T-Zellen, Eosinophile und Basophile Granulozyten durch Freisetzung von Chemokinen. M2-Makrophagen tragen zur Dämpfung von Entzündungen bei, fördern den Gewebeumbau und die Immunregulation. Der Metabolismus von Makrophagen ändert sich zusätzlich während der Polarisierung so dass M1-Makrophagen eine erhöhte Glykolyse durch Stabilisierung des Transkriptionsfaktors HIF-1 $\alpha$ und eine reduzierte mitochondriale Aktivität zeigen. Im Unterschied dazu schalten M2-Makrophagen auf eine hohe mitochondriale Aktivität und Arginin-Metabolismus um. Die Immunantwort auf nekroptotische Zellen und die Polarisierung von Makrophagen, die nekroptotische Zellen phagozytieren, ist noch nicht gut untersucht.

In den meisten Studien wird der Zelltod durch biologische oder chemische Verbindungen induziert, was zu Artefakten führen kann, indem die Makrophagen direkt beeinflusst werden und andere Signalwege als der Zelltod ausgelöst werden. Daher war es das Ziel des zweiten Teils dieser Arbeit unter Verwendung eines reinen Zelltodsystems die Immunantwort auf sterbende Zellen zu untersuchen. Dabei verwendeten wir ein reines Zelltodsystem, bei dem Apoptose oder Nekroptose durch induzierbare Proteininteraktionen ausgelöst wurden. Die chemische Induktion der Annäherung von Proteinen ist eine etablierte Methode um einen bestimmten biologischen Prozess in Zellen zu aktivieren, ohne dass es zu Nebenwirkungen kommt. Dies basiert auf der Bindung von Liganden (FK-506 oder Rapamycin) an FK-506-bindende Proteine (FKBP). Die chemische Induktion der Annäherung kann als Werkzeug verwendet werden, um die Dimerisierung oder Oligomerisierung von Zielproteinen $\mathrm{zu}$ induzieren, indem das anvisierte Protein mit einer oder mehreren modifizierten FKBP-Domänen fusioniert wird. Symmetrische Dimerisierung kann durch Behandlung mit einem bivalenten Liganden wie synthetischen Dimeren von FK-506 oder Rapamycin erreicht werden. Diese Liganden, wie der hier verwendete B/B-Homodimerisierer (AP20187), sind spezifisch für modifizierte FKBP-Domänen wie z.B. die FKBP F36V-Mutante und binden nicht an endogenes FKBP. In unserem Zelltodsystem verwendeten wir NIH 3T3-Zellen, die entweder dimerisierbare Caspase 8 oder oligomerisierbare RIPK3 exprimierten. Die 
Zugabe des Dimerisierers zu den Zellen führte zu Apoptose oder Nekroptose, was durch Caspase 3/7-Aktivierung, Phosphorylierung von MLKL bzw. Inhibitor-Experimente bestätigt wurde. Wir analysierten den Effekt der sterbenden Zellen auf peritoneale Makrophagen indem wir eine Ko-Kultur in einem Transwell-System etablierten. Das genetische Profil von Makrophagen, die mit sterbenden Zellen ko-kultiviert wurden, wurde durch RNA-Sequenzierung analysiert. In Makrophagen, die mit nekroptotischen Zellen ko-kultiviert wurden, waren Gene des Chemotaxis- und Hypoxie-Signalwegs hochreguliert. Der Hypoxie-Signalweg wird durch HIF-1 $\alpha$ vermittelt, das auch metabolische Veränderungen in polarisierten Makrophagen induziert. Wir konnten zeigen, dass Makrophagen, die mit nekroptotischen Zellen ko-kultiviert wurden, eine verringerte mitochondriale Atmung aufwiesen, was auf eine M1-Polarisation hinweist. Die Konzentration von CXCL1, das in der RNA-Sequenzierung erhöht war, war auch im Überstand von ko-kultivierten Makrophagen und von nekroptotischen Zellen hochreguliert, was zeigt, dass nekroptotische Zellen CXCL1 sezernieren, aber zusätzlich auch die Genexpression von CXCL1 in peritonealen Makrophagen induzieren.

Als nächstes haben wir unsere Ergebnisse in einem in vivo Modell der selbst-auflösenden Entzündung validiert. Wir wählten das Modell der Zymosan-A-induzierten Peritonitis in Mäusen. Hier führte die Injektion von $10 \mathrm{mg} / \mathrm{kg}$ Zymosan-A in das Peritoneum von C57BL/6 Mäusen $\mathrm{zu}$ einer selbstauflösenden Entzündung. Zymosan-A ist ein Hefebestandteil, der von Phagozyten erkannt wird. Die Phagozytose von Zymosan-A führt zur Aktivierung des Transkriptionsfaktors NF- $\mathrm{BB}$ und zur Freisetzung von proinflammatorischen Zytokinen und Chemokinen. Während der Zymosan-A-induzierten Peritontitis infiltrieren Neutrophile Granulozyten das Gewebe. Der Zustrom von Neutrophilen Granulozyten erreicht seinen Höhepunkt etwa nach 4 Stunden und die Entzündungsreaktion gipfelt an Tag 1. Mit der Rekrutierung von Makrophagen und der Phagozytose von Neutrophilen Granuolozyten erreicht die Auflösung der Entzündung an Tag 3 ihren Höhepunkt. Am Ende werden die pro-inflammatorischen Makrophagen zu anti-inflammatorischen Zellen umprogrammiert und die Entzündung ist nach 6 Tagen abgeklungen. Um die Rolle von Apoptose und Nekroptose bei der Auflösung der Entzündung zu analysieren, wurde Zymosan-A in das Peritoneum von C57 BL/6 Mäusen injiziert und nach 8 Stunden (direkt vor dem Höhepunkt der Entzündung) wurden entweder sterbende Zellen oder Zelltod-Inhibitoren injiziert. Apoptose oder Nekroptose wurden durch Injektion des Caspase-Inhibitors zVAD.fmk bzw. des RIPK3-Inhibitors 
GSK'872 inhibiert. Zellen, Zytokine und Chemokine wurden 1, 3 und 6 Tage nach Zymosan-A-Injektion durch Lavage des Peritoneums isoliert und analysiert.

Die Hemmung der Nekroptose während der Zymosan-A-induzierten Peritonits verringerte die Anzahl der Neutrophilen Granulozyten an Tag 1. Die Rekrutierung von Neutrophilen Granulozyten könnte durch die Inhibierung der Nekroptose und damit verbundenen niedrigeren Konzentrationen von CXCL1 im Peritoneum beeinflusst sein. Hier sind weitere Experimente nötig, um zu beweisen, dass die Hemmung der Nekroptose $\mathrm{zu}$ verringerten Konzentrationen von CXCL1 in vivo führt und dies für die reduzierte Anzahl von Neutrophilen Granulozyten im Peritoneum verantwortlich ist.

Die RNA-Sequenzierung von Makrophagen, die mit apoptotischen Zellen ko-kultiviert wurden, zeigte eine unerwartete Auswirkung auf die Makrophagenpolarisierung, da Signalwege des Zellzyklus und der Zellteilung erhöht waren. Die gesteigerte Proliferation der Makrophagen wurde durch zwei funktionelle Assays mit aus Mäusen isolierten peritonealen Makrophagen und IC-21-Makrophagen bestätigt. Die Hemmung der Apoptose während der Zymosan-A-induzierten Peritonits bei Mäusen zeigte verminderte mRNA-Level von Zellzyklus-Mediatoren in peritonealen Makrophagen. Gleichzeitig mit der Zellzyklusaktivierung wiesen die Daten der RNA-Sequenzierung auf die Aktivierung von $\mathrm{PGE}_{2}$-Signalwegen hin, was darauf hindeutet, dass apoptotische Zellen oder die Efferozytose apoptotischer Zellen die Proliferation von Makrophagen, vermittelt durch $\mathrm{PGE}_{2}$, induzieren.

Zusammenfassend konnten wir im zweiten Teil dieser Arbeit feststellen, dass apoptotische Zellen die Transkription von Zellzyklusgenen und die Proliferation von Makrophagen induzieren und nekroptotische Zellen in der Lage sind, das Chemokinprofil von Makrophagen und damit die Rekrutierung von Neutrophilen Granulozyten zu beeinflussen. 


\section{Literature}

1. D'Arcy, M.S., Cell death: a review of the major forms of apoptosis, necrosis and autophagy. Cell Biol Int, 2019. 43(6): p. 582-592.

2. Degterev, A. and J. Yuan, Expansion and evolution of cell death programmes. Nat Rev Mol Cell Biol, 2008. 9(5): p. 378-90.

3. Yuan, J. and H.R. Horvitz, A first insight into the molecular mechanisms of apoptosis. Cell, 2004. 116(2 Suppl): p. S53-6, 1 p following S59.

4. Elmore, S., Apoptosis: a review of programmed cell death. Toxicol Pathol, 2007. 35(4): p. 495-516.

5. Galluzzi, L., et al., Molecular mechanisms of cell death: recommendations of the Nomenclature Committee on Cell Death 2018. Cell Death Differ, 2018. 25(3): p. 486-541.

6. Gordon, S. and A. Pluddemann, Macrophage Clearance of Apoptotic Cells: A Critical Assessment. Front Immunol, 2018. 9: p. 127.

7. Poon, I.K., et al., Apoptotic cell clearance: basic biology and therapeutic potential. Nat Rev Immunol, 2014. 14(3): p. 166-80.

8. Sprooten, J., et al., Necroptosis in Immuno-Oncology and Cancer Immunotherapy. Cells, 2020. 9(8).

9. Czabotar, P.E., et al., Control of apoptosis by the BCL-2 protein family: implications for physiology and therapy. Nat Rev Mol Cell Biol, 2014. 15(1): p. 49-63.

10. Fuchs, Y. and H. Steller, Live to die another way: modes of programmed cell death and the signals emanating from dying cells. Nat Rev Mol Cell Biol, 2015. 16(6): p. 329-44.

11. Tummers, B. and D.R. Green, Caspase-8: regulating life and death. Immunol Rev, 2017. 277(1): p. 76-89.

12. Pasparakis, M. and P. Vandenabeele, Necroptosis and its role in inflammation. Nature, 2015. 517(7534): p. 311-20.

13. Vanden Berghe, T., et al., Regulated necrosis: the expanding network of nonapoptotic cell death pathways. Nat Rev Mol Cell Biol, 2014. 15(2): p. 135-47.

14. Cai, Z., et al., Plasma membrane translocation of trimerized MLKL protein is required for TNF-induced necroptosis. Nat Cell Biol, 2014. 16(1): p. 55-65. 
15. Chen, X., et al., Translocation of mixed lineage kinase domain-like protein to plasma membrane leads to necrotic cell death. Cell Res, 2014. 24(1): p. 105-21.

16. Dondelinger, Y., et al., MLKL compromises plasma membrane integrity by binding to phosphatidylinositol phosphates. Cell Rep, 2014. 7(4): p. 971-81.

17. Quarato, G., et al., Sequential Engagement of Distinct MLKL

Phosphatidylinositol-Binding Sites Executes Necroptosis. Mol Cell, 2016. 61(4): p. 589-601.

18. Wang, H., et al., Mixed lineage kinase domain-like protein MLKL causes necrotic membrane disruption upon phosphorylation by RIP3. Mol Cell, 2014. 54(1): p. 133-146.

19. Xia, B., et al., MLKL forms cation channels. Cell Res, 2016. 26(5): p. 517-28.

20. Gong, Y.N., et al., ESCRT-III Acts Downstream of MLKL to Regulate Necroptotic Cell Death and Its Consequences. Cell, 2017. 169(2): p. 286-300 e16.

21. Thapa, R.J., et al., Interferon-induced RIP1/RIP3-mediated necrosis requires PKR and is licensed by FADD and caspases. Proc Natl Acad Sci U S A, 2013. 110(33): p. E3109-18.

22. Stanton, B.Z., E.J. Chory, and G.R. Crabtree, Chemically induced proximity in biology and medicine. Science, 2018. 359(6380).

23. Kolos, J.M., et al., FKBP Ligands-Where We Are and Where to Go? Front Pharmacol, 2018. 9: p. 1425.

24. Orozco, S., et al., RIPK1 both positively and negatively regulates RIPK3 oligomerization and necroptosis. Cell Death Differ, 2014. 21(10): p. 1511-21.

25. Oberst, A., et al., Inducible dimerization and inducible cleavage reveal a requirement for both processes in caspase-8 activation. J Biol Chem, 2010. 285(22): p. 16632-42.

26. Yatim, N., et al., RIPK1 and NF-kappaB signaling in dying cells determines cross-priming of CD8(+) T cells. Science, 2015. 350(6258): p. 328-34.

27. Boada-Romero, E., et al., The clearance of dead cells by efferocytosis. Nat Rev Mol Cell Biol, 2020. 21(7): p. 398-414.

28. Kourtzelis, I., G. Hajishengallis, and T. Chavakis, Phagocytosis of Apoptotic Cells in Resolution of Inflammation. Front Immunol, 2020. 11: p. 553. 
29. Saas, P., E. Daguindau, and S. Perruche, Concise Review: Apoptotic Cell-Based Therapies-Rationale, Preclinical Results and Future Clinical Developments. Stem Cells, 2016. 34(6): p. 1464-73.

30. Westman, J., S. Grinstein, and P.E. Marques, Phagocytosis of Necrotic Debris at Sites of Injury and Inflammation. Front Immunol, 2019. 10: p. 3030.

31. Feehan, K.T. and D.W. Gilroy, Is Resolution the End of Inflammation? Trends Mol Med, 2019. 25(3): p. 198-214.

32. Fullerton, J.N. and D.W. Gilroy, Resolution of inflammation: a new therapeutic frontier. Nat Rev Drug Discov, 2016. 15(8): p. 551-67.

33. Serhan, C.N. and J. Savill, Resolution of inflammation: the beginning programs the end. Nat Immunol, 2005. 6(12): p. 1191-7.

34. Germolec, D.R., et al., Markers of Inflammation. Methods Mol Biol, 2018. 1803: p. 57-79.

35. Watanabe, S., et al., The role of macrophages in the resolution of inflammation. J Clin Invest, 2019. 129(7): p. 2619-2628.

36. Sansbury, B.E. and M. Spite, Resolution of Acute Inflammation and the Role of Resolvins in Immunity, Thrombosis, and Vascular Biology. Circ Res, 2016. 119(1): p. 113-30.

37. Shapouri-Moghaddam, A., et al., Macrophage plasticity, polarization, and function in health and disease. J Cell Physiol, 2018. 233(9): p. 6425-6440.

38. Thapa, B. and K. Lee, Metabolic influence on macrophage polarization and pathogenesis. BMB Rep, 2019. 52(6): p. 360-372.

39. Weigert, A., C. Jennewein, and B. Brune, The liaison between apoptotic cells and macrophages--the end programs the beginning. Biol Chem, 2009. 390(5-6): p. 379-90.

40. Sieweke, M.H. and J.E. Allen, Beyond stem cells: self-renewal of differentiated macrophages. Science, 2013. 342(6161): p. 1242974.

41. Schafer, K.A., The cell cycle: a review. Vet Pathol, 1998. 35(6): p. 461-78.

42. Vermeulen, K., D.R. Van Bockstaele, and Z.N. Berneman, The cell cycle: a review of regulation, deregulation and therapeutic targets in cancer. Cell Prolif, 2003. 36(3): p. 131-49.

43. Schoggins, J.W., Interferon-Stimulated Genes: What Do They All Do? Annu Rev Virol, 2019. 6(1): p. 567-584. 
44. Shaw, A.E., et al., Fundamental properties of the mammalian innate immune system revealed by multispecies comparison of type I interferon responses. PLoS Biol, 2017. 15(12): p. e2004086.

45. Lee, A.J. and A.A. Ashkar, The Dual Nature of Type I and Type II Interferons. Front Immunol, 2018. 9: p. 2061.

46. Feng, H., et al., Interferon regulatory factor 1 (IRF1) and anti-pathogen innate immune responses. PLoS Pathog, 2021. 17(1): p. e1009220.

47. Platanias, L.C., Mechanisms of type-I- and type-II-interferon-mediated signalling. Nat Rev Immunol, 2005. 5(5): p. 375-86.

48. Conlon, K.C., M.D. Miljkovic, and T.A. Waldmann, Cytokines in the Treatment of Cancer. J Interferon Cytokine Res, 2019. 39(1): p. 6-21.

49. Zaidi, M.R., The Interferon-Gamma Paradox in Cancer. J Interferon Cytokine Res, 2019. 39(1): p. 30-38.

50. Eden, E., et al., Discovering motifs in ranked lists of DNA sequences. PLoS Comput Biol, 2007. 3(3): p. e39.

51. Eden, E., et al., GOrilla: a tool for discovery and visualization of enriched GO terms in ranked gene lists. BMC Bioinformatics, 2009. 10: p. 48.

52. Mootha, V.K., et al., PGC-1alpha-responsive genes involved in oxidative phosphorylation are coordinately downregulated in human diabetes. Nat Genet, 2003. 34(3): p. 267-73.

53. Subramanian, A., et al., Gene set enrichment analysis: a knowledge-based approach for interpreting genome-wide expression profiles. Proc Natl Acad Sci U S A, 2005. 102(43): p. 15545-50.

54. Livak, K.J. and T.D. Schmittgen, Analysis of relative gene expression data using real-time quantitative PCR and the 2(-Delta Delta $\mathrm{C}(\mathrm{T}))$ Method. Methods, 2001. 25(4): p. 402-8.

55. Vistica, D.T., et al., Tetrazolium-based assays for cellular viability: a critical examination of selected parameters affecting formazan production. Cancer Res, 1991. 51(10): p. 2515-20.

56. Koo, G.B., et al., Methylation-dependent loss of RIP3 expression in cancer represses programmed necrosis in response to chemotherapeutics. Cell Res, 2015. 25(6): p. 707-25.

57. Koba, M. and J. Konopa, [Actinomycin D and its mechanisms of action]. Postepy Hig Med Dosw (Online), 2005. 59: p. 290-8. 
58. Chawla-Sarkar, M., et al., Apoptosis and interferons: role of interferonstimulated genes as mediators of apoptosis. Apoptosis, 2003. 8(3): p. 237-49.

59. Roca, H., et al., Apoptosis-induced CXCL5 accelerates inflammation and growth of prostate tumor metastases in bone. J Clin Invest, 2018. 128(1): p. 248266.

60. Divakaruni, A.S., et al., Analysis and interpretation of microplate-based oxygen consumption and pH data. Methods Enzymol, 2014. 547: p. 309-54.

61. Goodridge, H.S. and D.M. Underhill, Fungal Recognition by TLR2 and Dectin1. Handb Exp Pharmacol, 2008(183): p. 87-109.

62. Cash, J.L., G.E. White, and D.R. Greaves, Chapter 17. Zymosan-induced peritonitis as a simple experimental system for the study of inflammation. Methods Enzymol, 2009. 461: p. 379-96.

63. Bruce Bagwell, C., High-Dimensional Modeling for Cytometry: Building Rock Solid Models Using GemStone and Verity Cen-se' High-Definition t-SNE Mapping. Methods Mol Biol, 2018. 1678: p. 11-36.

64. Dillon, C.P., et al., RIPK1 blocks early postnatal lethality mediated by caspase-8 and RIPK3. Cell, 2014. 157(5): p. 1189-202.

65. Chen, J., et al., Interferon-gamma induces the cell surface exposure of phosphatidylserine by activating the protein MLKL in the absence of caspase- 8 activity. J Biol Chem, 2019. 294(32): p. 11994-12006.

66. Rodriguez, D.A., et al., Characterization of RIPK3-mediated phosphorylation of the activation loop of MLKL during necroptosis. Cell Death Differ, 2016. 23(1): p. 76-88.

67. Vitner, E.B., et al., Induction of the type I interferon response in neurological forms of Gaucher disease. J Neuroinflammation, 2016. 13(1): p. 104.

68. Tanzer, M.C., et al., Combination of IAP antagonist and IFNgamma activates novel caspase-10- and RIPK1-dependent cell death pathways. Cell Death Differ, 2017. 24(3): p. 481-491.

69. Gunther, C., et al., The pseudokinase MLKL mediates programmed hepatocellular necrosis independently of RIPK3 during hepatitis. J Clin Invest, 2016. 126(11): p. 4346-4360.

70. Thapa, R.J., et al., NF-kappaB protects cells from gamma interferon-induced RIP1-dependent necroptosis. Mol Cell Biol, 2011. 31(14): p. 2934-46. 
71. Igney, F.H. and P.H. Krammer, Death and anti-death: tumour resistance to apoptosis. Nat Rev Cancer, 2002. 2(4): p. 277-88.

72. Cekay, M.J., et al., Smac mimetics and type II interferon synergistically induce necroptosis in various cancer cell lines. Cancer Lett, 2017. 410: p. 228-237.

73. Lee, S. and K. Margolin, Cytokines in cancer immunotherapy. Cancers (Basel), 2011. 3(4): p. 3856-93.

74. Maher, S.G., et al., Interferon: cellular executioner or white knight? Curr Med Chem, 2007. 14(12): p. 1279-89.

75. Parker, B.S., J. Rautela, and P.J. Hertzog, Antitumour actions of interferons: implications for cancer therapy. Nat Rev Cancer, 2016. 16(3): p. 131-44.

76. Zaidi, M.R. and G. Merlino, The two faces of interferon-gamma in cancer. Clin Cancer Res, 2011. 17(19): p. 6118-24.

77. Sarhan, J., et al., Constitutive interferon signaling maintains critical threshold of MLKL expression to license necroptosis. Cell Death Differ, 2019. 26(2): p. 332347.

78. Muendlein, H.I., et al., Constitutive Interferon Attenuates RIPK1/3-Mediated Cytokine Translation. Cell Rep, 2020. 30(3): p. 699-713 e4.

79. Muzio, M., et al., An induced proximity model for caspase-8 activation. J Biol Chem, 1998. 273(5): p. 2926-30.

80. Tait, S.W., et al., Widespread mitochondrial depletion via mitophagy does not compromise necroptosis. Cell Rep, 2013. 5(4): p. 878-85.

81. Orning, P. and E. Lien, Multiple roles of caspase-8 in cell death, inflammation, and innate immunity. J Leukoc Biol, 2021. 109(1): p. 121-141.

82. Orozco, S. and A. Oberst, RIPK3 in cell death and inflammation: the good, the bad, and the ugly. Immunol Rev, 2017. 277(1): p. 102-112.

83. Newton, K., RIPK1 and RIPK3: critical regulators of inflammation and cell death. Trends Cell Biol, 2015. 25(6): p. 347-53.

84. Dhuriya, Y.K. and D. Sharma, Necroptosis: a regulated inflammatory mode of cell death. J Neuroinflammation, 2018. 15(1): p. 199.

85. Nakanishi, M. and D.W. Rosenberg, Multifaceted roles of PGE2 in inflammation and cancer. Semin Immunopathol, 2013. 35(2): p. 123-37.

86. Park, J.Y., M.H. Pillinger, and S.B. Abramson, Prostaglandin E2 synthesis and secretion: the role of PGE2 synthases. Clin Immunol, 2006. 119(3): p. 229-40. 
87. Li, F., et al., Apoptotic cells activate the "phoenix rising" pathway to promote wound healing and tissue regeneration. Sci Signal, 2010. 3(110): p. ra13.

88. Sergeeva, M.G., et al., Prostaglandin E2 biphasic control of lymphocyte proliferation: inhibition by picomolar concentrations. FEBS Lett, 1997. 418(3): p. 235-8.

89. Sanin, D.E., et al., Mitochondrial Membrane Potential Regulates Nuclear Gene Expression in Macrophages Exposed to Prostaglandin E2. Immunity, 2018. 49(6): p. 1021-1033 e6.

90. Penke, L.R., et al., PGE2 accounts for bidirectional changes in alveolar macrophage self-renewal with aging and smoking. Life Sci Alliance, 2020. $3(11)$.

91. Brouckaert, G., et al., Phagocytosis of necrotic cells by macrophages is phosphatidylserine dependent and does not induce inflammatory cytokine production. Mol Biol Cell, 2004. 15(3): p. 1089-100.

92. Morelli, A.E. and A.T. Larregina, Apoptotic cell-based therapies against transplant rejection: role of recipient's dendritic cells. Apoptosis, 2010. 15(9): p. 1083-97.

93. Armas-Gonzalez, E., et al., Role of CXCL13 and CCL20 in the recruitment of B cells to inflammatory foci in chronic arthritis. Arthritis Res Ther, 2018. 20(1): p. 114.

94. Kazanietz, M.G., M. Durando, and M. Cooke, CXCL13 and Its Receptor CXCR5 in Cancer: Inflammation, Immune Response, and Beyond. Front Endocrinol (Lausanne), 2019. 10: p. 471.

95. Geissmann, F. and E. Mass, A stratified myeloid system, the challenge of understanding macrophage diversity. Semin Immunol, 2015. 27(6): p. 353-6.

96. Hashimoto, D., et al., Tissue-resident macrophages self-maintain locally throughout adult life with minimal contribution from circulating monocytes. Immunity, 2013. 38(4): p. 792-804.

97. Schulz, C., et al., A lineage of myeloid cells independent of Myb and hematopoietic stem cells. Science, 2012. 336(6077): p. 86-90.

98. Yona, S., et al., Fate mapping reveals origins and dynamics of monocytes and tissue macrophages under homeostasis. Immunity, 2013. 38(1): p. 79-91.

99. Gentek, R., K. Molawi, and M.H. Sieweke, Tissue macrophage identity and selfrenewal. Immunol Rev, 2014. 262(1): p. 56-73. 
100. Roszer, T., Understanding the Biology of Self-Renewing Macrophages. Cells, 2018. 7(8).

101. Davies, L.C., et al., Distinct bone marrow-derived and tissue-resident macrophage lineages proliferate at key stages during inflammation. Nat Commun, 2013. 4: p. 1886.

102. Bosurgi, L., et al., Macrophage function in tissue repair and remodeling requires IL-4 or IL-13 with apoptotic cells. Science, 2017. 356(6342): p. 1072-1076.

103. Luo, Y., S.C. Tucker, and A. Casadevall, Fc- and complement-receptor activation stimulates cell cycle progression of macrophage cells from G1 to S. J Immunol, 2005. 174(11): p. 7226-33.

104. Reddy, S.M., et al., Phagocytosis of apoptotic cells by macrophages induces novel signaling events leading to cytokine-independent survival and inhibition of proliferation: activation of Akt and inhibition of extracellular signal-regulated kinases 1 and 2. J Immunol, 2002. 169(2): p. 702-13.

105. Fogarty, C.E., et al., Extracellular Reactive Oxygen Species Drive ApoptosisInduced Proliferation via Drosophila Macrophages. Curr Biol, 2016. 26(5): p. 575-84.

106. Perez-Garijo, A., When dying is not the end: Apoptotic caspases as drivers of proliferation. Semin Cell Dev Biol, 2018. 82: p. 86-95.

107. Murray, L.A., et al., TGF-beta driven lung fibrosis is macrophage dependent and blocked by Serum amyloid P. Int J Biochem Cell Biol, 2011. 43(1): p. 154-62.

108. Robbins, C.S., et al., Local proliferation dominates lesional macrophage accumulation in atherosclerosis. Nat Med, 2013. 19(9): p. 1166-72.

109. Wynn, T.A. and K.M. Vannella, Macrophages in Tissue Repair, Regeneration, and Fibrosis. Immunity, 2016. 44(3): p. 450-462.

110. Weigert, A., et al., Killing Is Not Enough: How Apoptosis Hijacks TumorAssociated Macrophages to Promote Cancer Progression. Adv Exp Med Biol, 2016. 930: p. 205-39.

111. Lee, J.W., et al., Hypoxia signaling in human diseases and therapeutic targets. Exp Mol Med, 2019. 51(6): p. 1-13.

112. Knuth, A.K., et al., Interferons Transcriptionally Up-Regulate MLKL Expression in Cancer Cells. Neoplasia, 2019. 21(1): p. 74-81.

113. Knuth, A.K., et al., Apoptotic Cells induce Proliferation of Peritoneal Macrophages. Int J Mol Sci, 2021. 22(5). 


\section{Appendix}

Table 23. Results for upregulation of MLKL in DNA microarrays using the platform Interferome.

\begin{tabular}{|c|c|c|c|c|c|}
\hline Dataset ID & $\begin{array}{c}\text { Fold } \\
\text { Change }\end{array}$ & $\begin{array}{c}\text { IFN } \\
\text { Typ } \\
\text { e }\end{array}$ & $\begin{array}{c}\text { Treatment } \\
\text { Time [h] }\end{array}$ & Probe ID & $\begin{array}{l}\text { In Interferome } \\
\text { deposited } \\
\text { publication }\end{array}$ \\
\hline \multirow[t]{2}{*}{147} & 3.20 & I & 4 & A_24_P185044 & PMID: \\
\hline & & & & & 25536401 \\
\hline \multirow[t]{2}{*}{315} & 2.50 & II & 6 & A_24_P185044 & no publication \\
\hline & & & & & deposited \\
\hline \multirow[t]{2}{*}{318} & 2.50 & II & 6 & A_24_P185044 & PMID: \\
\hline & & & & & 23801603 \\
\hline \multirow[t]{2}{*}{125} & 3.43 & I & 3 & A_23_P61050 & no publication \\
\hline & & & & & deposited \\
\hline \multirow[t]{2}{*}{147} & 3.20 & I & 4 & A_23_P61050 & PMID: \\
\hline & & & & & 25536401 \\
\hline \multirow[t]{2}{*}{174} & 2.01 & II & 20 & A_23_P61050 & PMID: \\
\hline & & & & & 27233968 \\
\hline \multirow[t]{2}{*}{226} & 2.52 & I & 6 & A_23_P61050 & no publication \\
\hline & & & & & deposited \\
\hline \multirow[t]{2}{*}{293} & 16.12 & II & 24 & A_23_P61050 & PMID: \\
\hline & & & & & 27770554 \\
\hline \multirow[t]{2}{*}{294} & 3.54 & I & 72 & A_23_P61050 & PMID: \\
\hline & & & & & 25414701 \\
\hline \multirow[t]{2}{*}{315} & 2.92 & II & 6 & A_23_P61050 & no publication \\
\hline & & & & & deposited \\
\hline
\end{tabular}




\begin{tabular}{|c|c|c|c|c|c|}
\hline 318 & 2.92 & II & 6 & A_23_P61050 & $\begin{array}{l}\text { PMID: } \\
23801603\end{array}$ \\
\hline 44 & 2.55 & $\mathrm{I}$ & 6 & 238025_at & $\begin{array}{l}\text { PMID: } \\
20483728\end{array}$ \\
\hline 67 & 5.38 & $\mathrm{I}$ & 6 & 238025_at & $\begin{array}{l}\text { PMID: } \\
22875473\end{array}$ \\
\hline 144 & 5.73 & II & 24 & 238025_at & $\begin{array}{l}\text { PMID: } \\
25168428\end{array}$ \\
\hline 185 & 2.79 & $\mathrm{I}$ & 16 & 238025_at & $\begin{array}{l}\text { PMID: } \\
24569457\end{array}$ \\
\hline 300 & 2.45 & $\mathrm{I}$ & 6 & 238025_at & $\begin{array}{l}\text { PMID: } \\
20805419\end{array}$ \\
\hline 305 & 2.29 & II & 1.5 & 238025_at & $\begin{array}{l}\text { PMID: } \\
22610275\end{array}$ \\
\hline 306 & 2.31 & $\mathrm{I}$ & 1.5 & 238025_at & $\begin{array}{l}\text { PMID: } \\
22610275\end{array}$ \\
\hline 75 & 2.03 & $\mathrm{I}$ & 16 & ILMN_1667825 & $\begin{array}{l}\text { PMID: } \\
23626809\end{array}$ \\
\hline 78 & 2.40 & I & 12 & ILMN_1667825 & $\begin{array}{l}\text { PMID: } \\
23045702\end{array}$ \\
\hline 81 & 2.18 & I & 4 & ILMN_1667825 & $\begin{array}{l}\text { PMID: } \\
23045702\end{array}$ \\
\hline 82 & 2.73 & II & 12 & ILMN_1667825 & $\begin{array}{l}\text { PMID: } \\
23045702\end{array}$ \\
\hline 83 & 2.70 & II & 24 & ILMN_1667825 & $\begin{array}{l}\text { PMID: } \\
23045702\end{array}$ \\
\hline
\end{tabular}




\begin{tabular}{|c|c|c|c|c|c|}
\hline 128 & 6.27 & II & 10 & ILMN_1667825 & $\begin{array}{l}\text { PMID: } \\
26424569\end{array}$ \\
\hline 129 & 7.06 & I & 10 & ILMN_1667825 & $\begin{array}{l}\text { PMID: } \\
26424569\end{array}$ \\
\hline 130 & 5.08 & I & 10 & ILMN_1667825 & $\begin{array}{l}\text { PMID: } \\
26424569\end{array}$ \\
\hline 131 & 6.86 & II & 10 & ILMN_1667825 & $\begin{array}{l}\text { PMID: } \\
26424569\end{array}$ \\
\hline 161 & 4.46 & I & 6 & ILMN_1667825 & $\begin{array}{l}\text { PMID: } \\
24065129\end{array}$ \\
\hline 162 & 4.98 & II & 6 & ILMN_1667825 & $\begin{array}{l}\text { PMID: } \\
24065129\end{array}$ \\
\hline 163 & 4.16 & I & 3 & ILMN_1667825 & $\begin{array}{l}\text { PMID: } \\
20956346\end{array}$ \\
\hline 275 & 6.42 & I & 8 & ILMN_1667825 & $\begin{array}{l}\text { PMID: } \\
22851595\end{array}$ \\
\hline 276 & 5.11 & I & 2 & ILMN_1667825 & $\begin{array}{l}\text { PMID: } \\
22851595\end{array}$ \\
\hline 277 & 5.84 & I & 8 & ILMN_1667825 & $\begin{array}{l}\text { PMID: } \\
22851595\end{array}$ \\
\hline 278 & 4.57 & I & 2 & ILMN_1667825 & $\begin{array}{l}\text { PMID: } \\
22851595\end{array}$ \\
\hline 280 & 2.13 & I & 48 & ILMN_1667825 & $\begin{array}{l}\text { no publication } \\
\text { deposited }\end{array}$ \\
\hline 281 & 2.23 & I & 48 & ILMN_1667825 & $\begin{array}{l}\text { no publication } \\
\text { deposited }\end{array}$ \\
\hline
\end{tabular}




\begin{tabular}{|c|c|c|c|c|c|}
\hline 284 & 4.04 & II & 6 & ILMN_1667825 & $\begin{array}{l}\text { PMID: } \\
26030458\end{array}$ \\
\hline 373 & 2.00 & I & 24 & ILMN_1667825 & $\begin{array}{l}\text { PMID: } \\
23633948\end{array}$ \\
\hline 374 & 2.45 & $\mathrm{I}$ & 6 & ILMN_1667825 & $\begin{array}{l}\text { PMID: } \\
23633948\end{array}$ \\
\hline 375 & 2.42 & I & 6 & ILMN_1667825 & $\begin{array}{l}\text { PMID: } \\
23633948\end{array}$ \\
\hline 78 & 2.13 & I & 12 & ILMN_1715863 & $\begin{array}{l}\text { PMID: } \\
23045702\end{array}$ \\
\hline 82 & 2.20 & II & 12 & ILMN_1715863 & $\begin{array}{l}\text { PMID: } \\
23045702\end{array}$ \\
\hline 128 & 5.03 & II & 10 & ILMN_1715863 & $\begin{array}{l}\text { PMID: } \\
26424569\end{array}$ \\
\hline 129 & 5.99 & I & 10 & ILMN_1715863 & $\begin{array}{l}\text { PMID: } \\
26424569\end{array}$ \\
\hline 130 & 4.01 & I & 10 & ILMN_1715863 & $\begin{array}{l}\text { PMID: } \\
26424569\end{array}$ \\
\hline 131 & 5.81 & II & 10 & ILMN_1715863 & $\begin{array}{l}\text { PMID: } \\
26424569\end{array}$ \\
\hline 275 & 4.76 & I & 8 & ILMN_1715863 & $\begin{array}{l}\text { PMID: } \\
22851595\end{array}$ \\
\hline 277 & 5.10 & I & 8 & ILMN_1715863 & $\begin{array}{l}\text { PMID: } \\
22851595\end{array}$ \\
\hline 278 & 3.02 & I & 2 & ILMN_1715863 & $\begin{array}{l}\text { PMID: } \\
22851595\end{array}$ \\
\hline
\end{tabular}




\begin{tabular}{|c|c|c|c|c|c|}
\hline 82 & 2.35 & II & 12 & ILMN_1737498 & $\begin{array}{l}\text { PMID: } \\
23045702\end{array}$ \\
\hline 83 & 2.38 & II & 24 & ILMN_1737498 & $\begin{array}{l}\text { PMID: } \\
23045702\end{array}$ \\
\hline 128 & 6.19 & II & 10 & ILMN_1737498 & $\begin{array}{l}\text { PMID: } \\
26424569\end{array}$ \\
\hline 129 & 7.52 & $\mathrm{I}$ & 10 & ILMN_1737498 & $\begin{array}{l}\text { PMID: } \\
26424569\end{array}$ \\
\hline 130 & 5.45 & I & 10 & ILMN_1737498 & $\begin{array}{l}\text { PMID: } \\
26424569\end{array}$ \\
\hline 131 & 5.50 & II & 10 & ILMN_1737498 & $\begin{array}{l}\text { PMID: } \\
26424569\end{array}$ \\
\hline 161 & 3.16 & I & 6 & ILMN_1737498 & $\begin{array}{l}\text { PMID: } \\
24065129\end{array}$ \\
\hline 162 & 3.31 & II & 6 & ILMN_1737498 & $\begin{array}{l}\text { PMID: } \\
24065129\end{array}$ \\
\hline 163 & 2.61 & I & 3 & ILMN_1737498 & $\begin{array}{l}\text { PMID: } \\
20956346\end{array}$ \\
\hline 275 & 5.57 & I & 8 & ILMN_1737498 & $\begin{array}{l}\text { PMID: } \\
22851595\end{array}$ \\
\hline 276 & 3.28 & I & 2 & ILMN_1737498 & $\begin{array}{l}\text { PMID: } \\
22851595\end{array}$ \\
\hline 277 & 3.96 & I & 8 & ILMN_1737498 & $\begin{array}{l}\text { PMID: } \\
22851595\end{array}$ \\
\hline 278 & 2.26 & $\mathrm{I}$ & 2 & ILMN_1737498 & $\begin{array}{l}\text { PMID: } \\
22851595\end{array}$ \\
\hline
\end{tabular}




\begin{tabular}{|c|c|c|c|c|c|}
\hline 143 & 4.33 & $\mathrm{I}$ & 6 & 8002778 & $\begin{array}{l}\text { PMID: } \\
22005912\end{array}$ \\
\hline 201 & 2.96 & $\mathrm{I}$ & 2 & 8002778 & $\begin{array}{l}\text { PMID: } \\
24561305\end{array}$ \\
\hline 202 & 2.20 & I & 6 & 8002778 & $\begin{array}{l}\text { PMID: } \\
24561305\end{array}$ \\
\hline 301 & 3.60 & I & 6 & 8002778 & $\begin{array}{l}\text { PMID: } \\
22677194\end{array}$ \\
\hline 302 & 4.29 & II & 24 & 8002778 & $\begin{array}{l}\text { PMID: } \\
22677194\end{array}$ \\
\hline 303 & 2.67 & I & 24 & 8002778 & $\begin{array}{l}\text { PMID: } \\
22677194\end{array}$ \\
\hline 304 & 2.84 & II & 6 & 8002778 & $\begin{array}{l}\text { PMID: } \\
22677194\end{array}$ \\
\hline 307 & 5.32 & II & 48 & 8002778 & $\begin{array}{l}\text { PMID: } \\
23150618\end{array}$ \\
\hline 308 & 2.65 & II & 48 & 8002778 & $\begin{array}{l}\text { PMID: } \\
23150618\end{array}$ \\
\hline
\end{tabular}




\section{Eidensstattliche Erklärung}

Ich, Anne-Kathrin Knuth, erkläre hiermit an Eides statt, dass ich die vorgelegte Dissertation mit dem Titel „Evaluation of cell death pathways during cancer and inflammation" selbständig angefertigt und mich anderer Hilfsmittel als der in ihr angegebenen nicht bedient habe, insbesondere, dass alle Entlehnungen aus anderen Schriften mit Angabe der betreffenden Schrift gekennzeichnet sind. Alle Beiträge von Kollegen und Kolleginnen werden in der Arbeit explizit erwähnt und sind im Folgenden nochmals aufgeführt. Das unten aufgeführte Material wurde im Rahmen von Forschungskooperationen erstellt:

- Figure 39. Co-culture with apoptotic cells leads to proliferation of primary peritoneal macrophages and Figure 40. Images of macrophages before and after co-cultivation with apoptotic cells. Kollaborationspartner: Zumer Naeem, Institute for Vascular Signaling, Molekulare Medizin, Goethe Universität Frankfurt; Beitrag des Kollaborationspartners: Durchführung der Messung am Incucyte und Datenanalyse; eigener Beitrag: Vorbereitung der Zellen, statistische Auswertung, Aufbereitung der Abbildungen.

- Figure 43. Maximal mitochondrial respiration in macrophages co-cultured with necroptotic cells is significantly reduced. Kollaborationspartner: Dr. Dominik Fuhrmann, Institut für Biochemie I, Goethe Universität Frankfurt; Beitrag des Kollaborationspartners: Durchführung der Messung und Datenanalyse; eigener Beitrag: Vorbereitung der Zellen, statistische Auswertung, Aufbereitung der Abbildungen.

Die folgenden Teile der Dissertation sind bereits veröffentlicht worden: Im Journal Neoplasia in 2019 [112]:

- $\quad$ Figure 8 A. Treatment with IFN $\gamma$ and zVAD.fmk induced cell death in EFM-192A cells but not in HeLa cells. Figure 11 A. IFN $\gamma$ induced necroptosis in EFM-192A cells.

- Figure 12. IFN $\gamma$ treatment increased MLKL and IRF1 mRNA and protein level over time. 
- Figure 13. Type 1 and 2 IFNs increased expression of MLKL in different cell lines.

- -Figure 14 A. mRNA level of RIPK1 and RIPK3 after IFN $\gamma$ treatment.

- Figure 15. Inhibition of transcription by Actinomycin D inhibts MLKL upregulation.

- Figure 16. Inhibition of caspases had no effect on IFN $\gamma$-dependent upregulation of MLKL.

- Figure 19. Double knockdown of IRF1 and STAT1 abolished MLKL upregulation.

- Figure 20. Knockout of IRF1 prevented IFN $\gamma$-induced MLKL upregulation.

Im International Journal of Molecular Sciences in 2021 [113]:

- Figure 22 A. Expression of FV-hCasp8 and mRIPK3-FV was confirmed at higher passages. Figure 24 A. Optimal time point to measure dimerizer-induced cell death was determined.

- Figure 25. Dimerizer treatment induced apoptosis in FV-hCasp8 cells.

- Figure 29. Co-culture of macrophages and apoptotic cells led to elevated levels of CXCL5 in the supernatant.Figure $30 \mathrm{C}$. mRNA level of four different macrophage polarization marker were changed after co-culture with dying NIH 3 T3 cells. Figure 32 A. PCA plots demonstrated clear separation of treatment groups after RNA sequencing.

- Figure 33 A. MA plots show the log fold change relative to mean expression.

- Figure 34 A. Heat maps and hierarchical clustering of normalized counts with base mean $>30$ and p-value $<0.05$ was performed using Morpheus. Figure 35. In macrophages co-cultured with apoptotic cells compared to control gene sets of cell cycle and replication were enriched after GSEA.

- Figure 38 A. Gene ontology analysis using GOrilla confirmed results of the GSEA analysis.

- Figure 39. Co-culture with apoptotic cells leads to proliferation of primary peritoneal macrophages.

- Figure 41. Co-culture with apoptotic cells leads to proliferation of IC-21 cells. Figure 49 A. Death of cells in the lavage was analyzed at day 1, 3 and 6 via Annexin PI staining. 
- Figure 54 A, C, E. Injection of cell death inhibitors led to changes in immune cell levels.

- Figure 57 F. Concentrations of different chemokines in the lavage of mice treated with cell death inhibitors were analyzed.

- Figure 60. mRNA level of cell cycle genes in macrophages at day 1 of ZymosanA-induced peritonitis and zVAD.fmk injection.

Ich versichere, die Grundsätze der guten wissenschaftlichen Praxis beachtet, und nicht die Hilfe einer kommerziellen Promotionsvermittlung in Anspruch genommen zu haben.

Frankfurt am Main, den

Anne-Kathrin Knuth 